LAURA VOLPES

\title{
ON THE INTERPLANETARY PROPERTIES AND EVOLUTION OF CME-DRIVEN SHOCKS
}

DiSSERTATION 



\title{
On the interplanetary properties and evolution of CME-driven shocks
}

\author{
Dissertation \\ for the award of the degree \\ "Doctor of Philosophy" (Ph.D.) Division of Mathematics and \\ Natural Sciences \\ of the Georg-August-Universität Göttingen \\ within the doctoral programme PROPHYS \\ of the Georg-August University School of Science (GAUSS)
}

\author{
submitted by \\ Laura Volpes \\ from \\ Marino (RM), Italy
}

Göttingen, 2016 


\section{Thesis committee}

Dr. Volker Bothmer, Institut für Astrophysik, Universität Göttingen

Prof. Dr. Ansgar Reiners, Institut für Astrophysik, Universität Göttingen

$\underline{\text { Referees }}$

Prof. Dr. Jörg Büchner, Max-Planck-Institut für Sonnensystemforschung

Prof. Dr. Stefan Dreizler, Institut für Astrophysik, Universität Göttingen

Additional members of the examination board:

Dr. Volker Bothmer, Institut für Astrophysik, Universität Göttingen

Prof. Dr. Gert Lube, Institut für Numerische und Angewandte Mathematik, Universität Göttingen

Prof. Dr. Wolfgang Glatzel, Institut für Astrophysik, Universität Göttingen

Prof. Dr. Wolfram Kollatschny, Institut für Astrophysik, Universität Göttingen 


\section{Abstract}

This work presents the analysis of eleven CME events which were observed by instruments of the Sun-Earth Connection Coronal and Heliospheric Investigation (SECCHI) imaging suite on board the NASA Solar TErrestrial RElations Observatory (STEREO) mission, launched in October 2006. The selected CMEs were chosen such that their projected speeds exceed $1000 \mathrm{~km} \mathrm{~s}^{-1}$. Five out of the eleven events drove interplanetary shocks with distinct signatures in white-light observations from both the coronagraph COR2 and the heliospheric imager HI1 on board STEREO.

For each event geometrical modelling via the Graduated Cylindrical Shell (GCS) model was performed in order to test the assumption of self-similar evolution for the CME. The performance of the GCS model at heliocentric distances higher than $15 R_{\odot}$, corresponding to the outer field of view of COR2, was tested. The results show that the GCS is appropriate in reproducing the observed CME geometry at higher heliocentric distances mainly for events with projected angular half-width $\omega$ such that $\omega<45^{\circ}-P A_{A P E X}$, with $P A_{A P E X}$ being the position angle of the CME apex. The GCS results, moreover, showed that all the modelled CMEs decelerated during their propagation. The average acceleration was found to lie in the range $-15.21 \mathrm{~m} \mathrm{~s}^{-2} \leq a \leq-1.13 \mathrm{~m} \mathrm{~s}^{-2}$. The observed CME morphology was preserved during the CME propagation.

The Stereoscopic Self-Similar Expansion Model (SSSEM) was used to infer the kinematics of the analysed CMEs, and, in the cases in which a shock was detected in time-elongation plots, of the shock itself. Arrival times and speeds at the Lagrangian point $L_{1}$ were inferred by assuming propagation at constant speed and at constant deceleration and compared to in-situ Advanced Composition Explorer (ACE) measurements. The precision of the arrival time estimates is compatible with the accuracy of the models employed, with the smallest errors being as low as 1.3 hours. In six out of the nine events detected by ACE the inclusion of deceleration produced the most accurate results.

Models for the shock location were employed in order to determine the temporal evolution of the shock standoff distance $\Delta$, the density compression ratio $\frac{\rho_{d}}{\rho_{u}}$, and the upstream Mach number $M$ for the five events in which both the CME and shock kinematics could be inferred.

The derived shock parameters were extrapolated to the location of ACE and compared to their values computed from in-situ plasma and magnetic field measurements. ACE data was available for four of the five shock events. The observed values lie in the ranges $15 R_{\odot} \leq \Delta \leq 38 R_{\odot}, 1.89 \leq \frac{\rho_{d}}{\rho_{u}} \leq 2.87$, and $1.8 \leq M \leq 5.8$. The standoff distance estimates yield the most precise results when constant deceleration is included in the CME and shock propagation. In this case the errors are below $40 \%$ of the observed values, with the better predictions yielding discrepancies as low as $10 \%$. For three out of the four shock events detected by ACE the precision in the compression 
ratio extrapolations for the linear and quadratic fits is comparable, of the order of $10 \%$. For the other event the inclusion of deceleration reduces the error by $40 \%$. The accuracy of the Mach number extrapolations is lower, ranging from $20 \%$ to $\simeq 70 \%$ in the worst-case, and with the linear fit yielding more precise predictions in three out of four events.

The analysis presented in this work confirms that CME-driven shocks can be detected in remote-sensing observations by coronagraphs and heliospheric imagers, and that their kinematics can be derived independently from the CME kinematics via the application of inverse modelling techniques. The results also show that multipoint space observations are necessary in order to achieve a sufficient precision for the independent analysis of CMEs and CME-driven shocks when using the methods employed in this work. The analysis of their interplanetary evolution, as well as of the shock standoff distance, suggests that deceleration plays an important role in their propagation. More accurate results for the arrival time predictions and the standoff distance extrapolation are obtained when the effects of deceleration are accounted for. To the best of the author's knowledge this is the first time that the separate CME and shock kinematics, as well as the temporal evolution of the shock parameters in the inner heliosphere are derived from the application of inverse modelling techniques. Finally, the application of the methods presented here to data gathered from upcoming space-based mission such as the ESA Solar Orbiter and the NASA Solar Probe Plus, to be launched in 2018, will provide an unprecedented tool to further verify the models presented here and to determine the CME-driven shock parameters at locations unexplored so far. 


\section{Contents}

1. Motivation 1

2. Introduction to Coronal Mass Ejections 9

2.1. Definition and observational properties . . . . . . . . . . . 11

2.2. Solar signatures of CME eruption . . . . . . . . . . . . . 14

2.3. Models for CME eruption . . . . . . . . . . . . . . . 15

2.3.1. Tether cutting mechanism . . . . . . . . . . . . . 15

2.3.2. Magnetic breakout model . . . . . . . . . . . 16

2.3.3. Emerging flux triggering mechanism . . . . . . . . . . . 16

2.3.4. Instability and catastrophe-related triggering mechanisms . . . . 17

2.4. CME propagation . . . . . . . . . . . . . . . . . . . . 18

2.5. CME and shocks . . . . . . . . . . . . . . . 19

2.5.1. Interplanetary consequences of CME-driven shocks . . . . . . 21

2.6. In-situ signatures . . . . . . . . . . . . . . . . . . . . . . . . . . . . . . . . . . .

2.7. Effects on Earth . . . . . . . . . . . . . 25

3. Basic Concepts of Magnetohydrodynamics 29

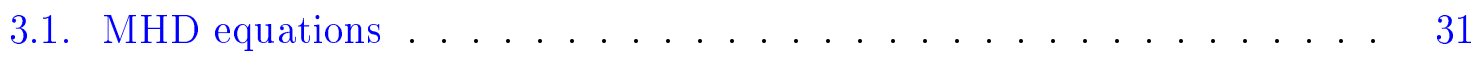

3.1.1. Frozen-in fields . . . . . . . . . . . . . 34

3.2. MHD waves .......................... 36

3.3. MHD discontinuities and shocks . . . . . . . . . . . . . 37

3.3.1. Contact and tangential discontinuities . . . . . . . . 39

3.3.2. Rotational discontinuities . . . . . . . . . . . . 40

3.3.3. Shocks .......................... 40

4. The NASA STEREO mission 43

4.1. The SECCHI suite . . . . . . . . . . . . . . . . 46

4.2. The other instruments on board STEREO . . . . . . . . . . . . . . . 49

4.3. Analysis of STEREO/SECCHI Observations . . . . . . . . . . . 49

5. On the Three Dimensional Structure of Coronal Mass Ejections $\quad 57$

5.1 . CMEs as cylindrical flux ropes . . . . . . . . . . . . . 59

5.2. The graduated cylindrical shell model . . . . . . . . . . . . . . . . . 61

5.3. Other geometrical models . . . . . . . . . . . . . . . . . 65

5.3.1. Circular cone model . . . . . . . . . . . 65

5.3.2. Elliptical cone model . . . . . . . . . . . . . 66 
6. Models for the CME kinematics $\quad 69$

6.1. Single Spacecraft Observations . . . . . . . . . . . . . . 71

6.1.1. The Fixed- $\phi$ Fitting Method . . . . . . . . . . . . . 71

6.1.2. The Harmonic Mean Fitting Method . . . . . . . . . . . . . . 72

6.1.3. The Self-Similar Expansion Fitting Method . . . . . . . . . 73

6.2. Multi Spacecraft Observations . . . . . . . . . . . . . . . 74

6.2.1. The Geometric Triangulation Method . . . . . . . . . . 75

6.2.2. The Tangent to a Sphere Method . . . . . . . . . . . . 76

6.2.3. The Stereoscopic Self-Similar Expansion model . . . . . . . . 76

7. Models for the Location of the Shock 81

7.0.4. Using the SSSEM to determine the CME-driven shock parameters 88

8. Event Analysis and Results 93

8.1. The 12 December $2008 \mathrm{CME}$. . . . . . . . . . . . . . . . . . 99

8.1.1. Solar source region . . . . . . . . . . . . . . . . . . 99

8.1.2. GCS modelling . . . . . . . . . . . . . . . . . 100

8.1.3. SSSEM results and in-situ comparison . . . . . . . . . . . 102

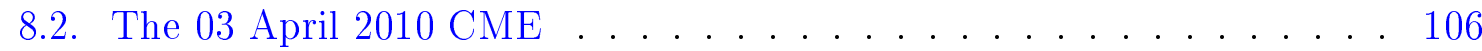

8.2.1. Solar source region . . . . . . . . . . . . . 106

8.2.2. GCS modelling . . . . . . . . . . . . . . 106

8.2.3. SSSEM results . . . . . . . . . . . . . . . . . . 108

8.2.4. CME-driven shock parameters . . . . . . . . . . . . . 111

8.2.5. In-situ comparison . . . . . . . . . . . . . . . . 112

8.2.6. Corrections for off-Ecliptic propagation . . . . . . . . . . 114

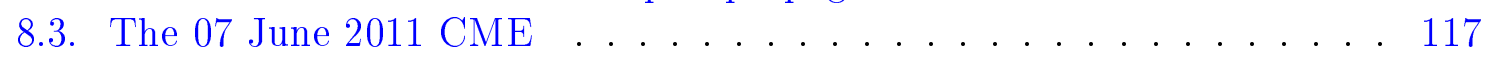

8.3.1. Solar source region . . . . . . . . . . . . . . . 117

8.3.2. GCS modelling . . . . . . . . . . . . . . 117

8.3.3. SSEF results . . . . . . . . . . . . . . 119

8.3.4. CME-driven shock parameters . . . . . . . . . . . 122

8.3.5. In-situ comparison . . . . . . . . . . . . . . . . . . . . . . . . . . . . . . . . . . . . . . .

8.4. The 03 August $2011 \mathrm{CME} \ldots \ldots \ldots \ldots \ldots \ldots . \ldots \ldots$

8.4.1. Solar source region . . . . . . . . . . . . . . 125

8.4.2. GCS modelling . . . . . . . . . . . . . 125

8.4.3. SSSEM results . . . . . . . . . . . . . . . 127

8.4.4. CME-driven shock parameters . . . . . . . . . . . . . . . . 129

8.4.5. In-situ comparison . . . . . . . . . . . . . . . . . 130

8.4.6. Corrections for off-Ecliptic propagation . . . . . . . . . . 132

8.5. The 22 October 2011 CME . . . . . . . . . . . . . . . 134

8.5.1. Solar source region . . . . . . . . . . . . . . . . . . . 134

8.5.2. GCS modelling . . . . . . . . . . . . . . . . 134

8.5.3. SSSEM results and in-situ comparison . . . . . . . . . . . 136

8.6. The 19 January 2012 CME . . . . . . . . . . . . . . . . . . . 141

8.6.1. Solar source region . . . . . . . . . . . . . . . 141

8.6.2. GCS modelling . . . . . . . . . . . . . . . . 141 
8.6.3. SSSEM results and in-situ comparison . . . . . . . . . . . 142

8.7. The 23 January 2012 CME . . . . . . . . . . . . . . . . 147

8.7.1. Solar source region . . . . . . . . . . . . . . 147

8.7.2. GCS modelling . . . . . . . . . . . . . . . . 147

8.7.3. SSSEM results and in-situ comparison . . . . . . . . . . . . . 149

8.8. The 07 March $2012 \mathrm{CME}$. . . . . . . . . . . . . . . . 153

8.8.1. Solar source region . . . . . . . . . . . . 153

8.8.2. GCS modelling . . . . . . . . . . . . . . . . 153

8.8.3. SSSEM results and in-situ comparison . . . . . . . . . . . . . 154

8.9. The 23 April 2012 CME . . . . . . . . . . . . . . . . . . 159

8.9.1. Solar source region . . . . . . . . . . . . . 159

8.9.2. SSSEM results and in-situ comparison . . . . . . . . . 160

8.10. The 12 July 2012 CME . . . . . . . . . . . . . . . . . . 164

8.10.1. Solar source region . . . . . . . . . . . . . . . . . . . 164

8.10.2. GCS modelling . . . . . . . . . . . . . 164

8.10.3. SSSEM results . . . . . . . . . . . . . 165

8.10.4. CME-driven shock parameters . . . . . . . . . . 168

8.10.5. In-situ comparison . . . . . . . . . . . . . . 168

8.10.6. Corrections for off-Ecliptic propagation . . . . . . . . . . . 171

8.11. The 15 March 2013 CME . . . . . . . . . . . . . . . . . 173

8.11.1. Solar source region . . . . . . . . . . . . . 173

8.11.2. GCS modelling . . . . . . . . . . . . . . . . 173

8.11.3. SSSEM results . . . . . . . . . . . . . . . 174

8.11.4. CME-driven shock parameters . . . . . . . . . . . . . . 177

8.11.5. In-situ comparison . . . . . . . . . . . . . . . . . . . 178

8.11.6. Corrections for off-Ecliptic propagation . . . . . . . . . 180

9. Discussion and conclusions 183

9.1. On the extension of the GCS model to heliospheric imagers observations 185

9.2. On the accuracy of the arrival times extrapolations . . . . . . . . . 188

9.3. On the accuracy of the shock parameter extrapolations . . . . . . . . 190

9.4. Conclusions and Outlook . . . . . . . . . . . . . 192

$\begin{array}{ll}\text { Appendices } & 197\end{array}$

$\begin{array}{ll}\text { A. Error propagation } & 199\end{array}$ 



\section{List of Figures}

1.1. SOHO composite AIA $304 \AA$ and LASCO C 2 observations of a CME on 04 January 2002. Image courtesy of the NASA/ESA SOHO consortium.

1.2. Left panel: STEREO/SECCHI COR2 B observation of a CME and its driven shock on 07 March 2011. Right panel: GCS modelling of the CME (red wireframe) and its driven shock (blue wireframe). The Sun lies behind the coronagraph occultor. Reprinted from Vourlidas \& Bemporad (2012) with the permission of AIP Publishing. . . . . . . .

2.1. $\mathrm{SOHO} / \mathrm{LASCO} \mathrm{C} 2$ image of a coronal mass ejection observed on December 2002. The three part structure of the CME is clearly visible in this event, and the Sun is behind the coronagraph occulter. Image courtesy of NASA . . . . . . . . . . . . . . . . . . .

2.2. SDO observations of the solar signatures of a CME, which erupted on 07 June 2011 from the SW quadrant of the solar disk. Top left: HMI magnetogram showing the magnetic field polarities and the CME source region. Top right: AIA EUV image at $193 \AA$ showing a flare and signatures of a prominence eruption associated with the CME. Bottom left: AIA EUV image at $304 \AA$ showing the prominence eruption. Bottom right: AIA EUV image at $171 \AA$ showing the post eruptive arcades. Images courtesy of NASA SDO consortium . . . . . . . . . .

2.3. Graphical representation of the tether cutting mechanism. From Chen (2011), adapted from Moore \& Labonte (1980). . . . . . . . . . . . . .

2.4. Graphical representation of the magnetic breakout model. From Chen (2011), adapted from Antiochos et al. (1999). . . . . . . . . . . .

2.5. Graphical representation of the emerging flux triggering mechanism. From Chen (2011), adapted from Chen \& Shibata (2000). . . . . . . . .

2.6. Time profile of the soft X-ray flux of a flare (solid line) and the velocity of the associated CME (dotted line), showing the 3-phase initiation scenario proposed by Zhang et al. (2001, 2004). Image from Zhang et al. (2001). (c)AAS. Reproduced with permission. . . . . . . . . . .

2.7. Left panel:SOHO/LASCO C2 observations of a CME-driven shock conforming to the bow shock morphology. The arrow indicates the whitelight signature of the bow shock. Adapted from Vourlidas et al. (2003). (C)AAS. Reproduced with permission. Right panel: SOHO/LASCO C2 observations of a CME-driven shock conforming to the double front morphology. The circle indicates the double front shock. Adapted from Vourlidas (2006). . . . . . . . . . . . . . . . 
2.8. Wind radio observations showing a type III and a type II radio burst, consequence of a fast CME which erupted at the Sun in September 2005. Image credit NASA/ESA. . . . . . . . . . . . . .

2.9. Helios 1 in-situ measurements of the solar wind revealing a shock wave on 19 July 1980, followed by a CME with unique magnetic cloud signatures. In the upper panel bidirectional electron flows can be seen in measurements of the flow angle $\phi_{E}$ of suprathermal electrons. The value $\phi_{E}=0^{\circ}$ corresponds to propagation in the anti-Sunward direction. Negative values of the pitch angles correspond to clockwise direction, whereas positive values correspond to counter clockwise propagation. Reprinted from Bothmer (1999) with the permission of AIP publishing.

3.1. Magnetic field lines through a surface $S$ enclosed by a curve $C$. From Spruit (2013) . . . . . . . . . . . . . .

3.2. Propagation speed of MHD waves as a function of the angle with respect to the direction of the magnetic field. The left panel shows the case in which the Alfven speed is larger than the sound speed, while the right panel illustrates the case in which the sound speed is larger than the Alfveń speed. Adapted from Baumjohann \& Treumann (1996). . . . . .

4.1. Position of the STEREO spacecraft in different mission phases. Image courtesy of the NASA STEREO consortium. . . . . . . . . . .

4.2. First light STEREO-B EUVI images taken on 12 December 2006. Image courtesy of the NASA STEREO/SECCHI consortium. . . . . . . .

4.3. Top panel: composite EUVI 195, COR1 and COR2 image of a CME erupted on 7 June 2011. Image credits: http://www. thesuntoday . org/ tag/solar-terrestrial-relations-observatory/. Bottom panel: STEREO A and B SECCHI complete field of view. The Earth is visible in the HI2 fields of view. Image credits: NASA STEREO/SECCHI consortium. . .

4.4. Top panel: Typical brightness of a CME compared to the cumulative brightness of the $\mathrm{K}$ and $\mathrm{F}$ corona as a function of angular distance from the Sun. The fields of view of the heliospheric imagers HI1 and HI2 are included. The intensity curves clearly show that the background coronal signal dominates over the signal produced by evolving CMEs. Bottom panel: subsequent steps necessary to extract the CME signal from HI1 and HI2 images. Reprinted from Eyles et al. (2009) with the permission of AIP publishing. Figure $4.4 \mathrm{~b}$ is a raw HI1 image taken on 25 January 2007, Figure 4.4c and is obtained by correcting for flatfield and shutterless readout and Figure $4.4 \mathrm{~d}$ is derived by removing blooming around planets. Figure 4.4e shows the signal of the underlying CME, extracted via background subtraction. Reprinted from Eyles et al. (2009) with the permission of AIP publishing. . . . . . . . . .

4.5. Left panel: running difference image (4.5c) built by subtracting two subsequent HI1 A snapshots of a propagating CME (4.5a and 4.5b). . . 
4.6. Time elongation plot obtained from STEREO A COR2, HI1 and HI2 observations between 18 and 22 January 2012 . . . . . . . . . .

4.7. Left panel: sketch of a CME seen as a point source moving at an angle $\delta$ from the plane of the Sky, seen by an observer at a distance $d$ from the Sun. Adapted from Sheeley et al. (1999) Right panel: elongation angle as a function of distance for different CME propagation angles (Top). Bottom: $\frac{d \epsilon}{d \rho}$ as a function of distance for different CME propagation angles. 54

5.1. Graphical representation of a CME seen as an expanding magnetic flux rope. . . . . . . . . . . . . . . . . .

5.2. Top panel: graphical representation of the appearance of front-sided CMEs in relation to the orientation of their source region's neutral line (indicated as NL in the picture). Bottom panel: SOHO/LASCO C2 observations of four CMEs (right) and their solar source regions (left) matching the scheme in Figure 5.2a. From Cremades \& Bothmer (2004). (C)ESO, reproduced with permission. . . . . . . . . . . .

5.3. Face-on (left) and edge-on (right) view of a flux rope modelled via the Graduated Cylindrical Shell model. From Thernisien (2011). CAAS. Reproduced with permission. . . . . . . . . . . . .

5.4. Orientation of the GCS flux rope in 3D. From Thernisien et al. (2006). (C)AAS. Reproduced with permission. . . . . . . . . . . .

5.5. Top panel: white-light (left) and synthetic (right) coronagraph images of four CMEs. The synthetic images are obtained via a GCS fit to the observed CME brightness profiles. Bottom panel: comparison between the observed electron density (solid line) and the one obtained by assigning a Gaussian profile to the GCS croissant (dashed line). From Thernisien et al. (2006). C AAS. Reproduced with permission. . . . . . . . . . .

5.6. Ice cream cone model for CMEs (Figure 5.6a, adapted from Zhao et al. (2002)), and its projection on the plane of the sky (Figure 5.6b, from Zhao et al. (2002)). . . . . . . . . . . . . . . .

5.7. Elliptical cone model for CMEs. Adapted from Cremades \& Bothmer (2005a) . . . . . . . . . . . . . . . . . .

6.1. Graphical representation of the fixed- $\phi$ approximation. Adapted from Sheeley et al. (1999) . . . . . . . . . . . . . . . .

6.2. Graphical representation of the harmonic mean approximation. Adapted from Lugaz et al. (2009). . . . . . . . . . . . . . . . . . . . .

6.3. Graphical representation of the self-similar expansion model. Adapted from Davies et al. (2012) . . . . . . . . . . . . . . . 73

6.4. Graphical representation of the geometrical triangulation method. Adapted from Liu et al. (2010a). . . . . . . . . . . . . . . . 75

6.5. Graphical representation of the tangent to a sphere approximation. Adapted from Lugaz et al. (2010). . . . . . . . . . . . . . . . . . 76

6.6. Graphical representation of the Stereoscopic Self-Similar Expansion Model. Adapted from Davies et al. (2013). . . . . . . . . . . . . . . . . 77 
6.7. In-situ arrival of a CME modelled with the stereoscopic self-similar expansion model. Adapted from Möstl \& Davies (2013). . . . . . . . . . .

7.1. Schematic representation of the formation of a shock in front of a blunt

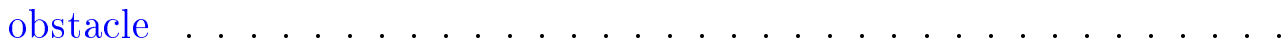

7.2. Comparison of the shock location obtained from model and experiments. Reprinted from Spreiter et al. (1966) with the permission of AIP publishing. . . . . . . . . . . . . . . . .

7.3. Friedrichs I diagrams for MHD waves in the cases $v_{A}>c_{s}$ and $v_{A}<c_{s}$. Adapted from Spreiter et al. (1966). . . . . . . . . . . . .

7.4. Left panel: Friedrichs II diagrams for MHD waves in the cases $v_{A}>c_{s}$. Right panel: Friedrichs II diagrams in the limits $v_{A} \ll c_{s}$ (top) and $v_{A} \gg c_{s}$ (bottom). Adapted from Spreiter et al. (1966) . . . . . . . .

7.5. Views of a CME modelled via the SSSEM in the Ecliptic plane (Figure 7.5a), and in a plane normal to the Ecliptic and containing the Sun-Earth line (Figure 7.5b). Adapted from Volpes \& Bothmer (2015). . . . . . .

8.1. SOHO MDI, EIT $198 \AA$ and EIT $304 \AA$ observations of the solar disk on 12 December 2008. Prominence material rising above the solar surface is seen in EIT $304 \AA$ images on the NW quadrant. Images courtesy of the NASA/ESA SOHO consortium. . . . . . . . . . . .

8.2. Top panel: positions of the STEREO spacecraft on 12 December 2008. Image credit: NASA STEREO consortium. Bottom panel: difference images obtained from STEREO/SECCHI A and B COR2 HI1 and HI2 observations between 12 and 14 December 2008, showing subsequent phases of the CME evolution. The top and bottom panel respectively show STEREO A and B images. The Earth is visible in the HI2 images. The planet in the inner HI2 A field of view is Venus. . . . . . . . . . . 100

8.3. GCS modelling for COR2 (upper panel) and HI1 (lower panels) for observations of the 12 December $2008 \mathrm{CME}$ at 14:22 UT and 22:49 UT. .

8.4. Top panel: j-maps obtained from STEREO A (left) and B (right) observations between 12 and 15 December 2008. The green dots represent the measured elongations used to derive the CME kinematics. Bottom panel: temporal evolution of the CME height, speed and direction of propagation towards Earth obtained by applying the SSSEM for $\lambda=0^{\circ}$

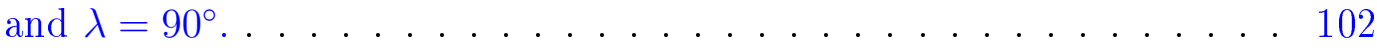

8.5. ACE in-situ plasma and magnetic field measurements between 14 and 18 December 2008. The purple area marks the ICME passage. Overplotted are the SSSEM arrival times for $\lambda=0^{\circ}$ and $\lambda=90^{\circ}$ obtained via linear and quadratic fit to the SSSEM height. . . . . . . . . . . . . 104

8.6. SOHO MDI, EUVI $193 \AA$ and EUVI $304 \AA$ observations of the solar disk on 03 April 2010. Images courtesy of the NASA SDO consortium. . . . 106 
8.7. Top panel: positions of the STEREO spacecraft on 03 April 2010. Image credit: NASA STEREO consortium. Bottom panel: difference images obtained from STEREO/SECCHI A (top panel) and B (bottom panel) COR2 HI1 and HI2 observations, showing subsequent phases of the CME evolution for the 03 April 2010 event. . . . . . . . . . . . .

8.8. GCS modelling for STEREO/SECCHI COR2 (upper panel) and HI1 (lower panels) observations of the 03 April $2010 \mathrm{CME}$ at 12:08 UT and 17:29 UT.

8.9. Top panel: j-maps obtained from STEREO A (left) and B (right) observations between 02 and 07 April 2010. The red dots represent the time-elongation measurements for the shock, and the green dots are the time-elongation pairs measured for the CME.

8.10. Time evolution of the CME height, speed and direction of propagation towards Earth obtained by applying the SSSEM for $\lambda=0^{\circ}$ and $\lambda=90^{\circ}$ to STEREO/SECCHI observations of the 03 April 2010 event. . . . . .

8.11. Time evolution of the shock height, speed and direction of propagation towards Earth obtained by applying the SSSEM for $\lambda=0^{\circ}$ and $\lambda=90^{\circ}$ for the 03 April 2010 event. . . . . . . . . . . . . . . . .

8.12. Time evolution of the CME radius of curvature (top left), the shock standoff distance (top right), the compression ratio (bottom left) and the Mach number (bottom right) obtained by applying the SSSEM for $\lambda=10^{\circ}$ and $\lambda=90^{\circ}$ to STEREO/SECCHI observations of the 03 April 2010 event. . . . . . . . . . . . . . . . . .

8.13. ACE in-situ plasma and magnetic field measurements between 04 and 07 April 2010. The blue area indicates the shock sheath region, and the purple area marks the CME passage. Overplotted are the SSSEM arrival times for $\lambda=0^{\circ}$ and $\lambda=90^{\circ}$ obtained via a quadratic fit to the CME and shock SSSEM heliocentric distance. . . . . . . . . . . . 8.14. SDO MDI, AIA $193 \AA$ and AIA $304 \AA$ observations of the solar disk on 07 June 2011. Images courtesy of the NASA SDO consortium. . . . . .

8.15. Top panel: positions of the STEREO spacecraft on 07 June 2011. Image credit: NASA STEREO consortium. Bottom panel: difference images obtained from STEREO/SECCHI A and B COR2 HI1 and HI2 observations, showing subsequent phases of the CME evolution for the 07 June 2011 event.

8.16. GCS modelling for STEREO/SECCHI COR2 (upper panel) and HI1 (lower panels) observations of the 07 June $2011 \mathrm{CME}$ at 08:15 UT and $14: 29$ UT. . . . . . . . . . . . . . . . .

8.17. Top panel: j-maps obtained from STEREO B observations between 06 and 11 June 2011. The red dots represent the time-elongation measurements for the shock, and the green dots are the time-elongation pairs measured for the CME. . . . . . . . . . . . . . . .

8.18. Time evolution of the CME height above the Sun obtained via the application of the SSEF technique for $\lambda=0^{\circ}$ and $\lambda=90^{\circ}$ to STEREO B observations of the 07 June 2011 event. . . . . . . . . . . . . 
8.19. Time evolution of the shock height above the Sun obtained via the application of the SSEF technique for $\lambda=0^{\circ}$ and $\lambda=90^{\circ}$ to STEREO B observations of the 07 June 2011 event. . . . . . . . . . . . . 121

8.20. Time evolution of the CME radius of curvature, the shock standoff distance, the compression ratio and the Mach number obtained applying the SSEF for $\lambda=10^{\circ}$ (red and orange dots) and $\lambda=90^{\circ}$ (blue dots) to STEREO-B observations of the 07 June 2011 event. . . . . . . . . . .

8.21. ACE in-situ plasma and magnetic field measurements between 07 and 10 June 2011. No signatures of the passage of a CME are present in the data. . . . . . . . . . . . . . . . . .

8.22. SDO HMI, AIA $193 \AA$ and $304 \AA$ observations of the solar disk on 03 August 2011. Images courtesy of the NASA SDO consortium. . . . . .

8.23. Top panel: positions of the STEREO spacecraft on 03 August 2011. Image credit: NASA STEREO consortium. Bottom panel: difference images obtained from STEREO/SECCHI A and B COR2 HI1 and HI2 observations, showing subsequent phases of the CME evolution for the 03 August 2011 event. . . . . . . . . . . . . . . . . .

8.24. GCS modelling for STEREO/SECCHI COR2 (upper panel) and HI1 (lower panels) observations of the 03 August $2011 \mathrm{CME}$ at 15:08 UT and $18: 49$ UT. . . . . . . . . . . . . . . .

8.25. Top panel: j-maps obtained from STEREO A (left) and B (right) observations between 04 and 08 August 2011. The red dots represent the time-elongation measurements for the shock, and the green dots are the time-elongation pairs measured for the CME. . . . . . . . . .

8.26. Time evolution of the CME height, speed and direction of propagation w.r.t. the Sun-Earth line obtained by applying the SSSEM for $\lambda=0^{\circ}$ and $\lambda=90^{\circ}$ to STEREO/SECCHI observations of the 03 August 2011 event.

8.27. Time evolution of the shock height, speed and direction of propagation towards Earth obtained by applying the SSSEM for $\lambda=0^{\circ}$ and $\lambda=90^{\circ}$ for the 03 August 2011 event. . . . . . . . . . . . . .

8.28. Time evolution of the CME radius of curvature, the shock standoff distance, the compression ratio and the Mach number obtained applying the SSSEM for $\lambda=10^{\circ}$ and $\lambda=90^{\circ}$ to STEREO/SECCHI observations of the 03 August 2011 event. . . . . . . . . . . . . . . .

8.29. ACE in-situ plasma and magnetic field measurements between 04 and 08 August 2011. The blue area indicates the shock sheath, and the purple area marks the CME passage. Overplotted are the SSSEM arrival times for $\lambda=0^{\circ}$ and $\lambda=90^{\circ}$ obtained via a quadratic fit to the CME and shock SSSEM heights.

8.30. SDO HMI, AIA $193 \AA$ and $304 \AA$ observations of the solar disk on 22 October 2011. Images courtesy of the NASA SDO consortium. . . . . 134 
8.31. Top panel: positions of the STEREO spacecraft on 22 October 2011. Image credit: NASA STEREO consortium. Bottom panel: difference images obtained from STEREO/SECCHI A and B COR2 HI1 and HI2 observations, showing subsequent phases of the CME evolution for the 22 October 2011 event. . . . . . . . . . . . . . . .

8.32. GCS modelling for STEREO/SECCHI COR2 (upper panel) and HI1 (lower panels) for observations of the 22 October 2011 CME at 13:08 UT and 16:49 UT.

8.33. Top panel: j-maps obtained from STEREO A (left) and B (right) observations between 22 and 27 October 2011. The green dots represent the measured elongations used to derive the CME kinematics. Bottom panel: temporal evolution of the CME height, speed and direction of propagation towards Earth obtained by applying the SSSEM for $\lambda=0^{\circ}$ and $\lambda=90^{\circ}$ to STEREO/SECCHI observations of the 22 October 2011 event.

8.34. ACE in-situ plasma and magnetic field measurements between 21 and 30 October 2011. The salmon area marks the CME passage. Overplotted are the SSSEM arrival times for $\lambda=0^{\circ}$ and $\lambda=90^{\circ}$ obtained via linear and quadratic fit to the SSSEM height for the 22 October 2011 event. .

8.35. SDO HMI, AIA $193 \AA$ and $304 \AA$ observations of the solar disk on 19 January 2012. Images courtesy of the NASA SDO consortium. . . . . . 141

8.36. Top panel: positions of the STEREO spacecraft on 19 January 2012. Image credit: NASA STEREO consortium. Bottom panel: difference images obtained from STEREO/SECCHI A and B COR2 HI1 and HI2 observations, showing subsequent phases of the CME evolution for the 19 January 2012 event. The top and bottom panel respectively show STEREO A and B images. . . . . . . . . . . . . . . .

8.37. GCS modelling for COR2 (upper panel) and HI1 (lower panels) for observations of the 19 January $2012 \mathrm{CME}$ at 16:08 UT and 22:49 UT. . .

8.38. Top panel: j-maps obtained from STEREO A (left) and B (right) observations between 18 and 22 January 2012. The green dots represent the measured elongations used to derive the CME kinematics. Bottom panel: temporal evolution of the CME height, speed and direction of propagation towards Earth obtained by applying the SSSEM for $\lambda=0^{\circ}$ and $\lambda=90^{\circ}$ to STEREO/SECCHI observations of the 19 January 2012

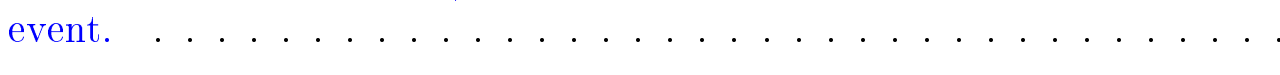

8.39. ACE in-situ plasma and magnetic field measurements between 18 and 24 January 2012. The purple marks the ICME passage. Overplotted are the SSSEM arrival times for $\lambda=0^{\circ}$ and $\lambda=90^{\circ}$ obtained via linear and quadratic fit to the SSSEM height. . . . . . . . . . . . .

8.40. SDO HMI, AIA $193 \AA$ and $304 \AA$ observations of the solar disk on 23 January 2012. Images courtesy of the NASA SDO consortium. . . . . . 147 
8.41. Top panel: positions of the STEREO spacecraft on 23 January 2012. Image credit: NASA STEREO consortium. Bottom panel: difference images obtained from STEREO/SECCHI A (top panel) and B (bottom panel) COR2 HI1 and HI2 observations, showing subsequent phases of the CME evolution for the 23 January 2012 event. The top and bottom panel respectively show STEREO A and B images. . . . . . . . . . . 148

8.42. GCS modelling for STEREO/SECCHI COR2 (upper panel) and HI1 (lower panels) for observations of the 23 January 2012 CME at 04:08 UT and 06:49 UT. . . . . . . . . . . . . . . .

8.43. Top panel: j-maps obtained from STEREO A (left) and B (right) observations between 22 and 25 January 2012. The green dots represent the measured elongations used to derive the CME kinematics. Bottom panel: temporal evolution of the CME height, speed and direction of propagation towards Earth obtained by applying the SSSEM for $\lambda=0^{\circ}$ and $\lambda=90^{\circ}$ to STEREO/SECCHI observations of the 23 January 2012 event.

8.44. ACE in-situ plasma and magnetic field measurements between 22 and 28 January 2012. The purple area marks the ICME passage. Overplotted are the SSSEM arrival times for $\lambda=0^{\circ}$ and $\lambda=90^{\circ}$ obtained via linear and quadratic fit to the SSSEM height. . . . . . . . . . .

8.45. SDO HMI magnetogram, and SDO AIA $193 \AA$ and $304 \AA$ EUV images of the solar disk on 07 March 2012. The brightening in the EUV images corresponds to the flare associated with the CME eruption. Images courtesy of the NASA SDO consortium. . . . . . . . . .

8.46. Top panel: positions of the STEREO spacecraft on 07 March 2012. Image credit: NASA STEREO consortium. Bottom panel: difference images obtained from STEREO/SECCHI A and B COR2 HI1 and HI2 observations, showing subsequent phases of the CME evolution for the 07 March 2012 event. . . . . . . . . . . . . . . . . . . . 154

8.47. GCS modelling for COR2 (upper panel) and HI1 (lower panels) for observations of the 07 March $2012 \mathrm{CME}$ at 14:22 UT and 22:49 UT. . .

8.48. Top panel: j-maps obtained from STEREO A (left) and B (right) observations between 06 and 10 March 2012. The green dots represent the measured elongations used to derive the CME kinematics. Bottom panel: temporal evolution of the CME height, speed and direction of propagation towards Earth obtained applying the SSSEM for $\lambda=0^{\circ}$ and $\lambda=90^{\circ}$ to STEREO/SECCHI observations of the 07 March 2012

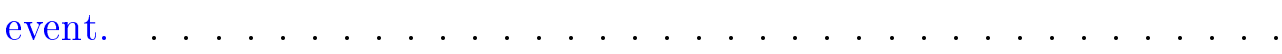

8.49. ACE in-situ plasma and magnetic field measurements between 06 and 13 March 2012. The purple area marks the CME passage. Overplotted are the SSSEM arrival times for $\lambda=0^{\circ}$ and $\lambda=90^{\circ}$ obtained via linear and quadratic fit to the SSSEM height. . . . . . . . . . .

8.50. SDO HMI, AIA $193 \AA$ and $304 \AA$ observations of the solar disk on 23 April 2012. Images courtesy of the NASA SDO consortium. . . . . . 159 
8.51. Top panel: positions of the STEREO spacecraft on 24 April 2012. Image credit: NASA STEREO consortium. Bottom panel: difference images obtained from STEREO/SECCHI A (top panel) and B (bottom panel) COR2 HI1 and HI2 observations, showing subsequent phases of the CME evolution for the 24 April 2012 event. . . . . . . . . . . . . . .

8.52. Top panel: j-maps obtained from STEREO A (left) and B (right) observations between 22 and 27 April 2012. The green dots represent the measured elongations used to derive the CME kinematics. Bottom panel: temporal evolution of the CME height, speed and direction of propagation towards Earth obtained by applying the SSSEM for $\lambda=0^{\circ}$ and $\lambda=90^{\circ}$ to STEREO/SECCHI observations of the 23 April 2012 event.

8.53. ACE in-situ plasma and magnetic field measurements between 24 and 27 April 2012. No signatures of the passage of an ICME are identified in the data. Overplotted are the SSSEM arrival times for $\lambda=0^{\circ}$ and $\lambda=90^{\circ}$ obtained via linear and quadratic fit to the SSSEM height. .

8.54. SDO HMI, AIA $193 \AA$ and $304 \AA$ observations of the solar disk on 12 July 2012. Images courtesy of the NASA SDO consortium. . . . . . . .

8.55. Top panel: positions of the STEREO spacecraft on 12 July 2012. Image credit: NASA STEREO consortium. Bottom panel: difference images obtained from STEREO/SECCHI A and B COR2 HI1 and HI2 observations, showing subsequent phases of the CME evolution for the 12 July 2012 event. . . . . . . . . . . . . . . . . . . .

8.56. GCS modelling for COR2 (upper panel) and HI1 (lower panels) for observations of the 12 July 2012 CME at 15:08 UT and 18:49 UT. . . . .

8.57. Top panel: j-maps obtained from STEREO A (left) and B (right) observations between 11 and 15 July 2012. The red dots represent the time-elongation measurements for the shock, and the green dots are the time-elongation pairs measured for the CME. . . . . . . . . .

8.58. Time evolution of the CME height, speed and direction of propagation towards Earth obtained by applying the SSSEM for $\lambda=0^{\circ}$ and $\lambda=90^{\circ}$ to STEREO/SECCHI observations of the 12 July 2012 event. . . . . .

8.59. Time evolution of the shock height, speed and direction of propagation towards Earth obtained by applying the SSSEM for $\lambda=0^{\circ}$ and $\lambda=90^{\circ}$ to STEREO/SECCHI observations of the 12 July 2012 event. . . . . . 168

8.60. Time evolution of the CME radius of curvature, the shock standoff distance, the compression ratio and the Mach number obtained applying the SSSEM for $\lambda=10^{\circ}$ and $\lambda=90^{\circ}$ to STEREO/SECCHI observations of the 12 July 2012 event. . . . . . . . . . . . . . . . . .

8.61. ACE in-situ plasma and magnetic field measurements between 13 and 17 July 2012. The blue area indicates the shock sheath region, and the purple area marks the CME passage. Overplotted are the SSSEM arrival times for $\lambda=0^{\circ}$ and $\lambda=90^{\circ}$ obtained via a quadratic fit to the CME and shock SSSEM heights. . . . . . . . . . . . . . 
8.62. SDO HMI, AIA $193 \AA$ and $304 \AA$ observations of the solar disk on 15 March 2013. Images courtesy of the NASA SDO consortium. . . . . . . 173

8.63. Top panel: positions of the STEREO spacecraft on 15 March 2013. Image credit: NASA STEREO consortium. Bottom panel: difference images obtained from STEREO/SECCHI A and B COR2 HI1 and HI2 observations, showing subsequent phases of the CME evolution for the 15 March 2013 event. . . . . . . . . . . . . . . . . . . .

8.64. GCS modelling for COR2 (upper panel) and HI1 (lower panels) for observations of the 15 March $2013 \mathrm{CME}$ at 15:08 UT and 18:49 UT. Comet PANNStar is visible in HI1B images. . . . . . . . . . . . . . .

8.65. Top panel: j-maps obtained from STEREO A (left) and B (right) observations between 15 and 18 March 2013. The red dots represent the time-elongation measurements for the shock, and the green dots are the time-elongation pairs measured for the CME. . . . . . . . . . . .

8.66. Time evolution of the CME height, speed and direction of propagation towards Earth obtained by applying the SSSEM for $\lambda=0^{\circ}$ and $\lambda=90^{\circ}$ to STEREO/SECCHI observations of the 15 March 2013 event. . . . .

8.67. Time evolution of the shock height, speed and direction of propagation towards Earth obtained by applying the SSSEM for $\lambda=0^{\circ}$ and $\lambda=90^{\circ}$ to STEREO/SECCHI observations of the 15 March 2013 event. . . . .

8.68. Time evolution of the CME radius of curvature, the shock standoff distance, the compression ratio and the Mach number obtained by applying the SSSEM for $\lambda=10^{\circ}$ and $\lambda=90^{\circ}$ to STEREO/SECCHI observations of the 15 March 2013 event. . . . . . . . . . . . . . . . . .

8.69. ACE in-situ plasma and magnetic field measurements between 15 and 21 March 2013. The blue area indicates the shock sheath, and the purple area marks the CME passage. Overplotted are the SSSEM arrival times for $\lambda=0^{\circ}$ and $\lambda=90^{\circ}$ obtained via a quadratic fit to the CME and shock SSSEM heights. . . . . . . . . . . . . . .

9.1. Top panel: STEREO/SECCHI COR2 and HI1 fields of view showing the opening of the HI1 field of view in terms of position angles (PAs). The Ecliptic plane and the line $P A=0^{\circ}$ are marked in red. Bottom panel: STEREO/SECCHI HI1A difference images of the 03 August 2011 (right) and the 23 January 2012 (left) events. Lines of constant position angle are plotted over the images. Although the apex inclination with respect to the Ecliptic plane (red horizontal line) was similar for the two events, due to their different projected angular width, the 03 August 2011 event was imaged by HI1 in its entirety, while a large fraction of the 23 January 2012 lies outside the field of view of the instrument. . .

9.2. Difference between the observed and predicted arrival times al $L_{1}$ for the 9 events observed in-situ. Positive values correspond to early predictions. Results are presented for the linear and quadratic fits to the CME and, when available, shock height derived from the application of the SSSEM for $\lambda=0^{\circ}$ and $\lambda=90^{\circ} \ldots \ldots \ldots \ldots \ldots$ 
9.3. Error for the SSSEM standoff distance normalized to the observed value. Results are reported for the linear and the quadratic fits to the CME and shock SSSEM heights. The plotted values referred to the best fits to observations for the 03 April 2010, the 03 August 2011, the 12 July 2012 and the 15 March 2013 events. . . . . . . . . . . . . . . 191

9.4. Relative error for the standoff distance, compression ratio, and Mach number compared to observations. Results are reported for the linear and the quadratic fits to the CME and shock SSSEM heights. The plotted values referred to the best fits to observations for the 03 April 2010, the 03 August 2011, the 12 July 2012 and the 15 March 2013 events.192

9.5. Sketch representing a CME and its driven shock in different stages of their evolution. The picture summarises the results found in this work, namely that the standoff distance increases as the CME travels away from the Sun, and that deceleration occurs during its interplanetary

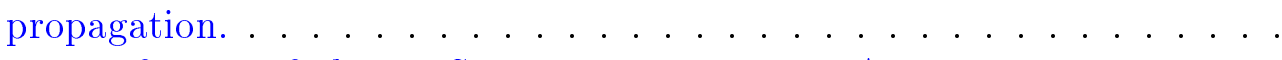

9.6. Field of view of the WISPR cameras at 9.86 AU superimposed on a composite STEREO/SECCHI COR and HI1 images. Reprinted from Vourlidas et al. (2015) with the permission of AIP publishing . . . . . . 195 



\section{List of Tables}

2.1. Classification of solar flares according to the peak of associated X-ray emission. From https://www.unc.edu/ rowlett/units/scales/solar_

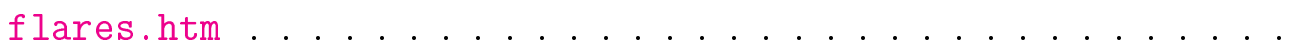

8.1. List of the events investigated in this work. The dates are in format YYYY-MM-DD, and refer to the date of CME eruption. For each event information is given on whether signatures of the CME-driven shocks were identified in white-light images and j-maps, and thus whether the shock parameters could be determined, whether it was detected by ACE, and if data-driven MHD simulations can be performed based on the source region location. . . . . . . . . . . . .

8.2. GCS modelling results for the 12 December 2008 CME. The table shows the imaging instrument, the time of observations, and the GCS parameters: Carrington longitude, heliographic latitude and tilt angle of the solar source region, flux rope height $R_{G C S}$, aspect ratio $k$, half-angle $\alpha$, and radial width $a=k R_{G C S} \ldots \ldots \ldots \ldots$

8.3. Observed CME eruption time, arrival time and speed at $L_{1}$ compared to those obtained via a linear (top panel) and quadratic (bottom panel) fit to the CME height-time profile derived by applying the SSSEM for $\lambda=0^{\circ}$ and $\lambda=90^{\circ}$ for the 12 December 2008 event. . . . . . . . .

8.4. GCS modelling results for the 03 April 2010 CME. The table shows the imaging instrument, the time of observations, and the GCS parameters: Carrington longitude, heliographic latitude and tilt angle of the solar source region, flux rope height $R_{G C S}$, aspect ratio $k$, half-angle $\alpha$, and radial width $a=k R_{G C S} \ldots \ldots \ldots \ldots \ldots$

8.5. Observed CME eruption time, arrival time and speed at $L_{1}$ compared to those obtained via a linear (top panel) and quadratic (bottom panel) fit to the CME height-time profile derived by applying the SSSEM for $\lambda=0^{\circ}$ and $\lambda=90^{\circ}$ for the 03 April 2010 event. . . . . . . . . . . .

8.6. Comparison between standoff distance, compression ratio, and Mach number for the 03 April 2010 event as derived from ACE in-situ data, and from the linear and quadratic fit to the SSSEM heights for $\lambda=10^{\circ}$

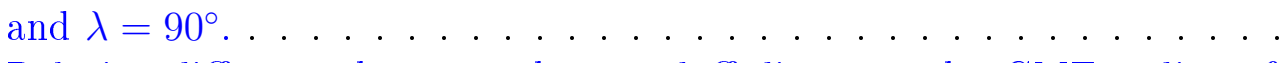

8.7. Relative difference between the standoff distance, the CME radius of curvature, the compression ratio and the Mach number computed on the Ecliptic plane and at the CME apex for the 03 April 2010 event. The results for the value of lambda which yields the best fit to the insitu shock parameters are shown. . . . . . . . . . . . . . 11 
8.8. GCS modelling results for the 07 June 2011 CME. The table shows the imaging instrument, the time of observations, and the GCS parameters: Carrington longitude, heliographic latitude and tilt angle of the solar source region, flux rope height $R_{G C S}$, aspect ratio $k$, half-angle $\alpha$, and radial width $a=k R_{G C S} \ldots \ldots \ldots \ldots \ldots$

8.9. CME speed and direction of propagation with respect to the Sun-Earth line obtained via the application of the SSEF technique for $\lambda=0^{\circ}$ and $\lambda=90^{\circ}$ STEREO B observations of the 07 June 2011 event. . . . . . .

8.10. Shock speed and direction of propagation with respect to the Sun-Earth line obtained via the application of the SSEF technique of the 07 June 2011 event for $\lambda=0^{\circ}$ and $\lambda=90^{\circ}$ to STEREO B observations of the 07 June 2011 event.

8.11. GCS modelling results for the 03 August $2011 \mathrm{CME}$. The table shows the imaging instrument, the time of observations, and the GCS parameters: Carrington longitude, heliographic latitude and tilt angle of the solar source region, flux rope height $R_{G C S}$, aspect ratio $k$, half-angle $\alpha$, and radial width $a=k R_{G C S} \ldots \ldots \ldots \ldots \ldots$

8.12. Observed CME eruption time, arrival time and speed at $L_{1}$ compared to those obtained via a linear (top panel) and quadratic (bottom panel) fit to the CME height-time profile derived by applying the SSSEM for $\lambda=0^{\circ}$ and $\lambda=90^{\circ}$ for the 03 August 2011 event. . . . . . . . . .

8.13. Comparison between standoff distance, compression ratio, and Mach number for the 03 August 2011 event as derived from ACE in-situ data, and from the linear and quadratic fit to the SSSEM heights for $\lambda=10^{\circ}$

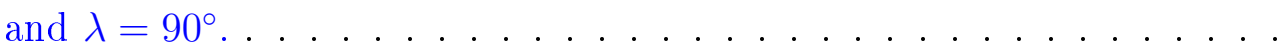

8.14. Relative difference between the standoff distance, the CME radius of curvature, the compression ratio and the Mach number computed on the Ecliptic plane and at the CME apex for the 03 August 2011 event. The results for the value of lambda which yields the best fit to the in-situ shock parameters are shown. . . . . . . . . . . . .

8.15. GCS modelling results for the 22 October $2011 \mathrm{CME}$. The table shows the imaging instrument, the time of observations, and the GCS parameters: Carrington longitude, heliographic latitude and tilt angle of the solar source region, flux rope height $R_{G C S}$, aspect ratio $k$, half-angle $\alpha$, and radial width $a=k R_{G C S} \ldots \ldots \ldots \ldots$

8.16. Observed CME eruption time, arrival time and speed at $L_{1}$ compared to those obtained via a linear (top panel) and quadratic (bottom panel) fit to the CME height-time profile derived applying the SSSEM for $\lambda=0^{\circ}$

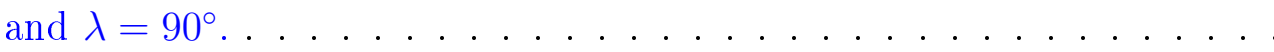

8.17. GCS modelling results for the 19 January 2012 CME. The table shows the imaging instrument, the time of observations, and the GCS parameters: Carrington longitude, heliographic latitude and tilt angle of the solar source region, flux rope height $R_{G C S}$, aspect ratio $k$, half-angle $\alpha$, and radial width $a=k R_{G C S} \ldots \ldots \ldots \ldots . \ldots \ldots$ 
8.18. Observed CME eruption time, arrival time and speed at $L_{1}$ compared to those obtained via a linear (top panel) and quadratic (bottom panel) fit to the CME height-time profile derived by applying the SSSEM for $\lambda=0^{\circ}$ and $\lambda=90^{\circ}$ to STEREO/SECCHI observations of the 19 January 2012 event.

8.19. GCS modelling results for the 23 January 2012 CME. The table shows the imaging instrument, the time of observations, and the GCS parameters: Carrington longitude, heliographic latitude and tilt angle of the solar source region, flux rope height $R_{G C S}$, aspect ratio $k$, half-angle $\alpha$, and radial width $a=k R_{G C S} \ldots \ldots \ldots \ldots$

8.20. Observed CME eruption time, arrival time and speed at $L_{1}$ compared to those obtained via a linear (top panel) and quadratic (bottom panel) fit to the CME height-time profile derived by applying the SSSEM for $\lambda=0^{\circ}$ and $\lambda=90^{\circ}$ to STEREO/SECCHI observations of the 23 January 2012 event.

8.21. GCS modelling results for the 07 March $2012 \mathrm{CME}$. The table shows the imaging instrument, the time of observations, and the GCS parameters: Carrington longitude, heliographic latitude and tilt angle of the solar source region, flux rope height $R_{G C S}$, aspect ratio $k$, half-angle $\alpha$, and radial width $a=k R_{G C S} \ldots \ldots \ldots \ldots \ldots$

8.22. Observed CME eruption time, arrival time and speed at $L_{1}$ compared to those obtained via a linear (top panel) and quadratic (bottom panel) fit to the CME height-time profile derived by applying the SSSEM for $\lambda=0^{\circ}$ and $\lambda=90^{\circ}$ to STEREO/SECCHI observations of the 07 March 2012 event. . . . . . . . . . . . . . . . .

8.23. Observed CME eruption time, arrival time and speed at $L_{1}$ compared to those obtained via a linear (top panel) and quadratic (bottom panel) fit to the CME height-time profile derived applying the SSSEM for $\lambda=0^{\circ}$ and $\lambda=90^{\circ}$ for the 23 April 2012 event. . . . . . . . . . .

8.24. GCS modelling results for the 12 July 2012 CME. The table shows the imaging instrument, the time of observations, and the GCS parameters: Carrington longitude, heliographic latitude and tilt angle of the solar source region, flux rope height $R_{G C S}$, aspect ratio $k$, half-angle $\alpha$, and radial width $a=k R_{G C S} \ldots \ldots \ldots \ldots \ldots$

8.25. Observed CME eruption time, arrival time and speed at $L_{1}$ compared to those obtained via a linear (top panel) and quadratic (bottom panel) fit to the CME height-time profile derived applying the SSSEM for $\lambda=0^{\circ}$ and $\lambda=90^{\circ}$ to STEREO/SECCHI observations of the 12 July 2012 event.169

8.26. Comparison between standoff distance, compression ratio, and Mach number for the 12 July 2012 event as derived from in-situ data, and from the linear and quadratic fits to the SSSEM heights for $\lambda=10^{\circ}$ and $\lambda=90^{\circ}$ 
8.27. Relative difference between the standoff distance, the CME radius of curvature, the compression ratio and the Mach number computed on the Ecliptic plane and at the CME apex for the 12 July 2012 event. The results for the value of lambda which yields the best fit to the in-situ shock parameters are shown. . . . . . . . . . . . .

8.28. GCS modelling results for the 15 March $2013 \mathrm{CME}$. The table shows the imaging instrument, the time of observations, and the GCS parameters: Carrington longitude, heliographic latitude and tilt angle of the solar source region, flux rope height $R_{G C S}$, aspect ratio $k$, half-angle $\alpha$, and radial width $a=k R_{G C S} \ldots \ldots \ldots \ldots \ldots$

8.29. Observed CME eruption time, arrival time and speed at $L_{1}$ compared to those obtained via a linear (top panel) and quadratic (bottom panel) fit to the CME height-time profile derived by applying the SSSEM for $\lambda=0^{\circ}$ and $\lambda=90^{\circ}$ to STEREO/SECCHI observations of the 15 March 2013 event. . . . . . . . . . . . . . . . .

8.30. Comparison between standoff distance, compression ratio, and Mach number for the 15 March 2013 event as derived from in-situ data, and from the linear and quadratic fit to the SSSEM heights for $\lambda=10^{\circ}$ and $\lambda=90^{\circ} \ldots \ldots \ldots \ldots \ldots \ldots \ldots \ldots$

8.31. Relative difference between the standoff distance, the CME radius of curvature, the compression ratio and the Mach number computed on the Ecliptic plane and at the CME apex for the 15 March 2013 event. The results for the value of lambda which yields the best fit to the in-situ shock parameters are shown. . . . . . . . . . . 180

9.1. Average errors in the arrival time predictions obtained via a linear (top panel) and quadratic (bottom panel) fit to the CME and, when possible, shock height-time profile derived by applying the SSSEM for $\lambda=0^{\circ}$ and $\lambda=90^{\circ}$ to the events analysed in this study. . . . . . . . . 189 


\section{Motivation}



Coronal mass ejections (CMEs) are transient events in which large amounts of plasma and magnetic field are expelled from the Sun into interplanetary space. CMEs are known for their space weather effects on Earth, being the major driver of the strongest geomagnetic storms (Bothmer \& Daglis, 2007, Schwenn et al., 2005). They are observed in remote-sensing white-light images (see Figure 1.1) and detected by in-situ instruments thanks to their peculiar plasma and magnetic field signatures. However, because the corona is optically thin, remote-sensing images only present a projection of the true 3D structure on the plane-of-the-sky. For these reasons properties like the CME geometry and its position and orientation in space are hard to infer (see e.g. Webb \& Howard 2012).

Several models have been proposed to reproduce the observed CME morphology. The Graduated Cylindrical Shell (GCS) model (Thernisien, 2011, Thernisien et al., 2006) is based on the assumptions that CMEs are force-free cylindrical flux ropes, and that their visual appearance is a consequence of the orientation of their source region's polar inversion line (PIL, Cremades \& Bothmer 2004). It represents CMEs as cylindrical fronts of varying cross-section supported by two conical legs. The flux rope is assumed to expand self-similarly.

Furthermore, it is of interest for space weather purposes and for the understanding of the interplanetary forces acting on CMEs, to be able to characterize their evolution and derive parameters such as their distance from the Sun, their direction of propagation and their speed. Several models have been developed in order to compute the de-projected speeds, heights and direction of propagation from remote-sensing observations of CMEs, which only present a projected view on the plane-of-the-sky. Such models were initially developed for single viewpoint observations and involve fitting the measured time-elongation ${ }^{1}$ profiles to a function which depends on the assumed CME geometry (Davies et al., 2012, Howard \& Tappin, 2009, Lugaz et al., 2009, Möstl \& Davies, 2013, Sheeley et al., 1999, 2008). Such fits are performed by imposing that the CME propagates radially and at constant speed. They yield the CME speed, direction of propagation along the Sun-Earth line and the temporal evolution of the CME distance from the Sun. The application of such models allows the determination of arrival times and speeds at $L_{1}{ }^{2}$. Such predictions can be compared to in-situ measurement of the solar wind taken at the same location by spacecraft like the Advanced Composition Explorer (ACE, Stone et al. 1998). A study on the accuracy of the single spacecraft fitting techniques performed by Möstl et al. (2014) revealed that the arrival times are predicted with average errors $\Delta t=8 \pm 6$ hours, but can be as high as 24 hours. The predicted arrival speeds are typically larger than the observed ones, with average errors $\Delta v=284 \pm 288 \mathrm{~km} \mathrm{~s}^{-1}$. For the faster events the arrival speed difference can be as high as $1000 \mathrm{~km} \mathrm{~s}^{-1}$. Such discrepancies are most likely associated with the assumption of propagation at constant speed intrinsic to the fitting methods (Lugaz \& Kintner, 2013).

The launch of the NASA Solar TErrestrial RElations Observatory (STEREO, Kaiser

\footnotetext{
${ }^{1}$ The elongation of a feature in white-light images is defined as the angular separation between the feature itself and the Sun.

${ }^{2}$ The Lagrangian point $L_{1}$ is located $1.5 \cdot 10^{6} \mathrm{~km}$ away from Earth along the Sun-Earth line
} 


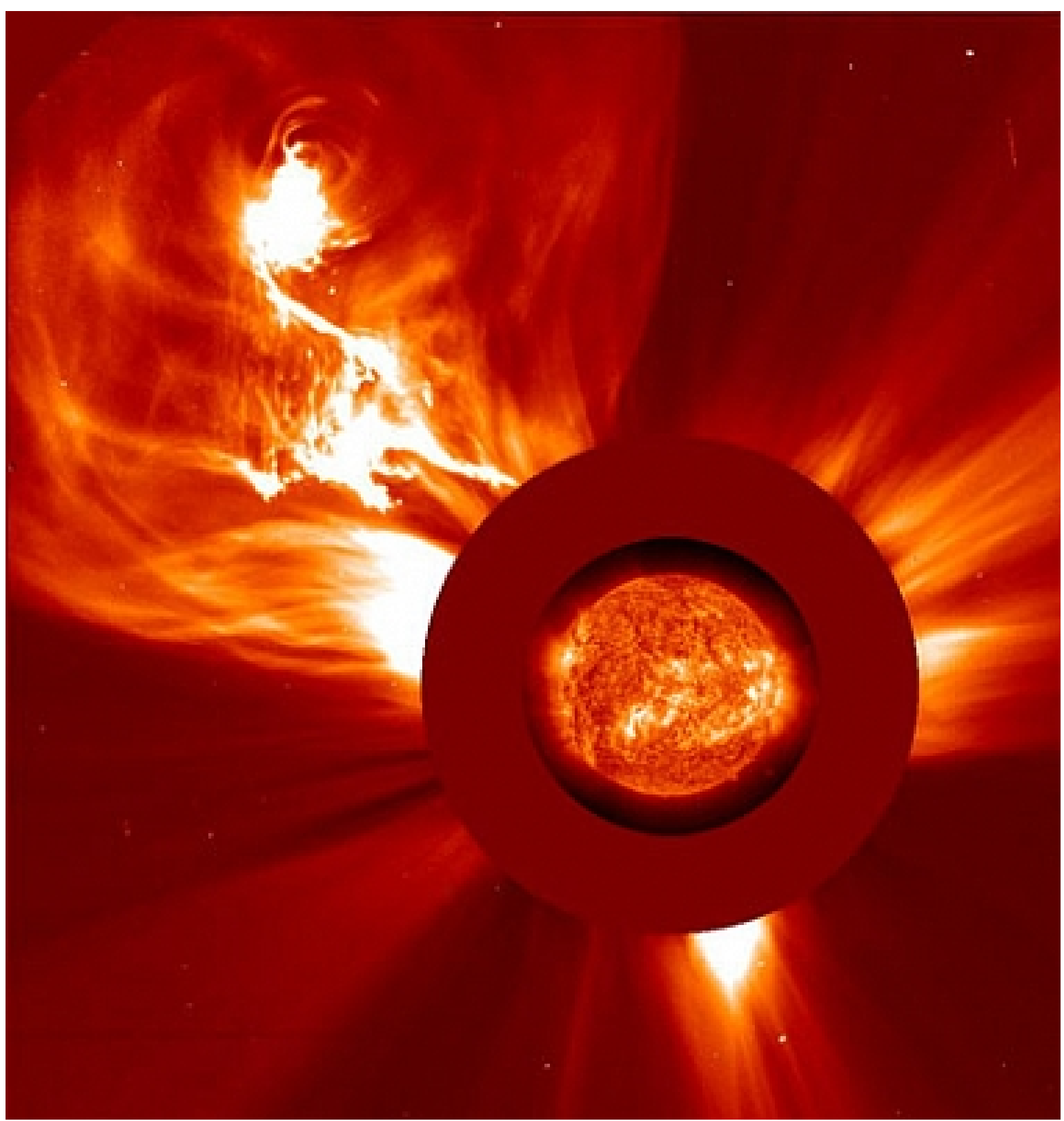

Figure 1.1.: SOHO composite AIA $304 \AA$ and LASCO C2 observations of a CME on 04 January 2002. Image courtesy of the NASA/ESA SOHO consortium.

et al. 2008) spacecraft in October 2006 provided for the first time simultaneous multipoint observations of coronal mass ejections. Each of the two spacecraft, STEREO A and $\mathrm{B}$, is equipped with an identical set of instruments. They orbit the Sun at 1 AU, and their angular separation increases by $\simeq 44^{\circ}$ per year. The Sun-Earth Connection Coronal and Heliospheric Investigation (SECCHI, Howard et al. 2008) imaging suite, mounted on both STEREO A and B, consists of five instruments: EUVI, an extreme ultraviolet imager, COR1 and COR2, two nested coronagraphs whose combined fields of view cover the range between 1.5 and $15 R_{\odot}$, and the heliospheric imagers HI1 and HI2, which monitor the inner heliosphere up to distances beyond $1 \mathrm{AU}$. The instru- 
ments on board the SECCHI suite provide observations of solar wind structures at locations not imaged before.

STEREO/SECCHI observations allow a continuous monitoring of the evolution of CMEs from the Sun all the way to Earth. They can be used to determine if significant distortions in the CME morphology take place during the later stages of their propagation as a consequence, for example, of their interaction with the ambient solar wind. The GCS model introduced above has been widely employed in order to reproduce the observed CME morphology in coronagraph images, i.e. up to distances of the order of $\simeq 15-20 R_{\odot}$ (see Bosman et al. 2012).

In the present work GCS fits obtained in the COR2 field of view were extended to HI1 observations, i.e. up to $\simeq 87 R_{\odot}$ in the plane-of-the-sky, in order to address the following questions:

- Can the GCS represent the observed CME geometry at distances from the Sun larger than $15 R_{\odot}$ ?

- Do CMEs expand self-similarly during their interplanetary evolution or does their geometry significantly change as a consequence of the action of interplanetary forces?

Stereoscopic observations of solar wind transients reduce the uncertainties not only in the models for the CME geometry, but also in the determination of kinematic of parameters like height, speed, and direction of propagation. The early models for the CME kinematics were adapted to work with simultaneous observations from different viewpoints (Davies et al., 2013, Liu et al., 2010a, Lugaz et al., 2010). The additional viewpoint provided by STEREO removes the necessity of imposing propagation at constant speed and at fixed direction. Such constraint is necessary for CME kinematics models based on the analysis of single spacecraft observations. Multipoint observationbased models have been widely and successfully employed to predict the CME arrival times and speeds at Earth (see, e.g. Liu et al. 2013). They are a useful tool for space weather forecasting and for the characterization of the interplanetary evolution of solar wind transients (Colaninno et al., 2013).

Unless the CME can be followed all the way to Earth, however, arrival time predictions are possible only via assumptions on the interplanetary propagation of the CME. The simplest hypothesis is that CMEs travel at constant speed. However, observational evidence indicates that CMEs interact with the solar wind and are consequently accelerated or decelerated depending on their speed relative to the solar wind speed (Gopalswamy et al., 2000). In particular, CMEs with speeds exceeding the solar wind speed are slowed down, whereas CMEs with speeds slower then the solar wind speed are accelerated. Following such observational evidence, arrival times at Earth can be estimated by assuming that the velocity changes linearly with time. A comparison with plasma and magnetic field measurement gathered by ACE at $L_{1}$ can help to verify the results. The analysis of the kinematic of 11 CMEs was performed in this work in order to answer the following questions:

- Is the precision of the arrival time and speed estimates improved by the use of multipoint observations-based models for the CME kinematics? 
- Does assuming that acceleration or deceleration occurs in the interplanetary propagation of CMEs improve the arrival time and speed estimates?

Finally, fast CMEs, i.e. CMEs which travel with speeds higher than the local Alfvén speed, drive interplanetary (IP) shocks. IP shocks accelerate particles in events known as Solar Energetic Particles (SEP) events (Reames, 1999). They compress and distort the interplanetary magnetic field (IMF), potentially contributing to the initiation of geomagnetic storms when inducing a strong component of the IMF anti-parallel to the Earth's magnetic field (Bothmer \& Schwenn, 1998, Schwenn et al., 2005).

IP shocks have been observed in-situ already since the 1980s, and CMEs were identified as their drivers by Sheeley et al. (1985). Thanks to the improved sensitivity of the available imaging instruments signatures of CME-driven shocks can now be detected not only in in-situ measurements but also in white-light images (Vourlidas et al. 2003, also see Figure 1.2). The detectability of a shock in remote-sensing observations depends on the strength of the background signal, the thickness of the shock along the line of sight, and the upstream density (Vourlidas, 2006). The combination of such observations with models relating the shock standoff distance to the density compression and the upstream Mach number allows the determination of such parameters at locations not accessible otherwise. Such models were originally developed to determine the location of the terrestrial bow shock (Seiff, 1962, Spreiter et al., 1966) under the assumption of high upstream Mach numbers. They were subsequently modified to extend their validity in the low Mach number regime (Farris \& Russell, 1994). The evolution of
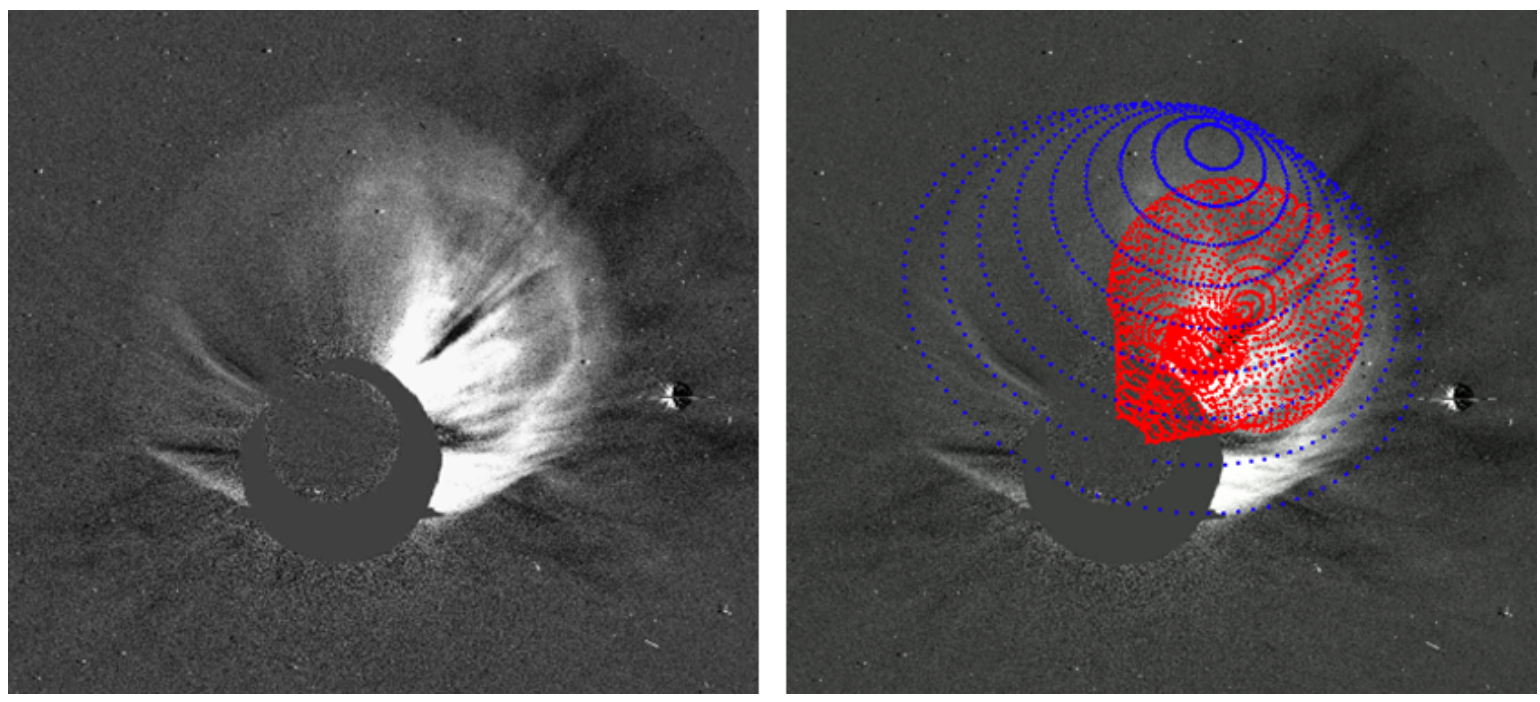

Figure 1.2.: Left panel: STEREO/SECCHI COR2 B observation of a CME and its driven shock on 07 March 2011. Right panel: GCS modelling of the CME (red wireframe) and its driven shock (blue wireframe). The Sun lies behind the coronagraph occultor. Reprinted from Vourlidas \& Bemporad (2012) with the permission of AIP Publishing.

the shock parameters based on remote-sensing observations of CMEs and CME-driven shocks was already studied in the works by Gopalswamy \& Yashiro (2011), Kim et al. (2012), Maloney \& Gallagher (2011), Poomvises et al. (2012). The authors employed 
forward modelling techniques to determine the parameters necessary to the calculation of the compression ratio and the Mach number. Under the assumption of low solar wind Mach number Gopalswamy \& Yashiro (2011), Kim et al. (2012), Poomvises et al. (2012) provided estimates for the coronal magnetic field. Such results are of particular relevance as currently there is no direct method to measure the coronal magnetic fields. In the present work STEREO COR2, HI1 and HI2 stereoscopic observations of coronal mass ejections were analysed. Signatures of CME-driven shock were searched for in the images in order to address the following questions:

- Up to which distances can CME-driven shocks be detected in remote-sensing observations?

- Can inverse modelling techniques be used to determine the separate evolution of CMEs and CME-driven shocks detected in remote-sensing observations?

- What are the interplanetary properties of CME-driven shock parameters and how do they evolve with time?

- Can the arrival times of CMEs and CME-driven shocks at the location of ACE be determined individually and with state-of-the-art-precision?

- Do the extrapolations of the shock parameters at the location of ACE compare well to their values derived from plasma and magnetic field measurements?

From a space weather perspective, an understanding of the properties of CME-driven shocks is crucial as SEPs are mainly accelerated by the shocks themselves, and parameters like speed and density compression could be used in simulations of particle acceleration(Lugaz et al., 2005, Vourlidas \& Ontiveros, 2009). CME-CME interaction seems to be correlated to SEP-rich events, and it involves plasma heating, field compression, possible disappearance of the trailing shock and strengthening of the leading shock (Gopalswamy et al., 2002). The ability to derive the shock properties from remote-sensing observations not only of "standalone" CMEs, but also of interacting CMEs will provide observational constraints for models and simulations of a wide variety of circumstances.

Upcoming space-based missions like the ESA Solar Orbiter (Müller et al., 2013) and the NASA Solar Probe Plus (Fox et al., 2015), to be launched in 2018, will provide data to further expand the methods developed in this work. The Solar Orbiter spacecraft will orbit the Sun at distances as close as 0.28 AU and with a maximum heliolatitude ${ }^{3}$ of $25^{\circ}$. The Solar Probe Plus mission will reach distances as close as $9.86 R_{\odot}$ from Sun centre. Such missions will monitor the interplanetary space via a combination of remote-sensing observations and in-situ measurements in the inner heliosphere and in the near-Sun environment. The application of the methods presented here to remotesensing observations will allow the investigation of the properties of CME-driven shocks and their spatial variation up to distances unexplored so far. The high cadence and sensitivity of the Wide-Field Imager for Solar Probe Plus (WISPR, Vourlidas et al.

\footnotetext{
${ }^{3}$ The inclination of the spacecraft's orbit with respect to the solar equator
} 
(2015)) cameras, moreover, will allow a consistent monitoring of CME-driven shock in white-light images. The combined in-situ measurements, finally, will provide an effective tool to test the results and the validity of the models for the shock location in the context of CME-driven shocks.

\section{Thesis outline}

The present work is structured as follows: Chapter 2 introduces coronal mass ejections and their driven shocks. It describes the observational properties of CMEs and introduces models for their eruption and interplanetary propagation. Radio and white-light observations of CME-driven shocks are discussed, as well as their interplanetary consequences. The chapter concludes with a description of the in-situ signatures of CMEs and CME-driven shocks and their impact on space weather.

Chapter 3 provides an overview of the ideal MHD equations and their derivation. MHD waves are introduced and the properties of MHD discontinuities and shocks are discussed.

The NASA STEREO mission and its instruments are presented in Chapter 4, with particular focus on the SECCHI imaging suite. The chapter also provides a description of the image processing techniques applied to STEREO/SECCHI observations and of the tools needed for the analysis of the evolution of solar wind transients.

Chapters 5, 6 and 7 present the models employed in this work. Chapter 5 introduces geometrical models of CMEs, with special emphasis on the Graduated Cylindrical Shell model. The models for the derivation of the kinematics of CMEs and CME-driven shocks are described in Chapter 6. Chapter 7 presents models for the computation of the shock parameters and their application to STEREO/SECCHI observations.

The employed methods and derived results are presented in Chapter 8. Chapter 9 provides a discussion of the results, followed by an outlook on the application of the methods presented here to future space missions. 
2. Introduction to Coronal Mass Ejections 

This chapter provides an introduction to coronal mass ejections. It describes their observational features and the phenomena accompanying their eruptions. Models for the genesis of CMEs are reviewed in Section 2.3, followed by a discussion on the interplanetary propagation of CMEs. The second part of the chapter focuses on CME-driven shocks and their signature in white-light observations, radio measurements and particles fluxes. Plasma and magnetic field measurements of the solar wind from in-situ spacecraft are discussed, and the in-situ signatures of shocks and CMEs are described. The chapter concludes with a discussion of the space weather effects of CMEs and CME-driven shocks.

\subsection{Definition and observational properties}

Solar coronal mass ejections (CMEs) are impulsive events that release magnetized plasma in interplanetary space. They originate from bipolar active regions, more complex active regions, or quiescent filaments (Cremades \& Bothmer, 2004, Gopalswamy,

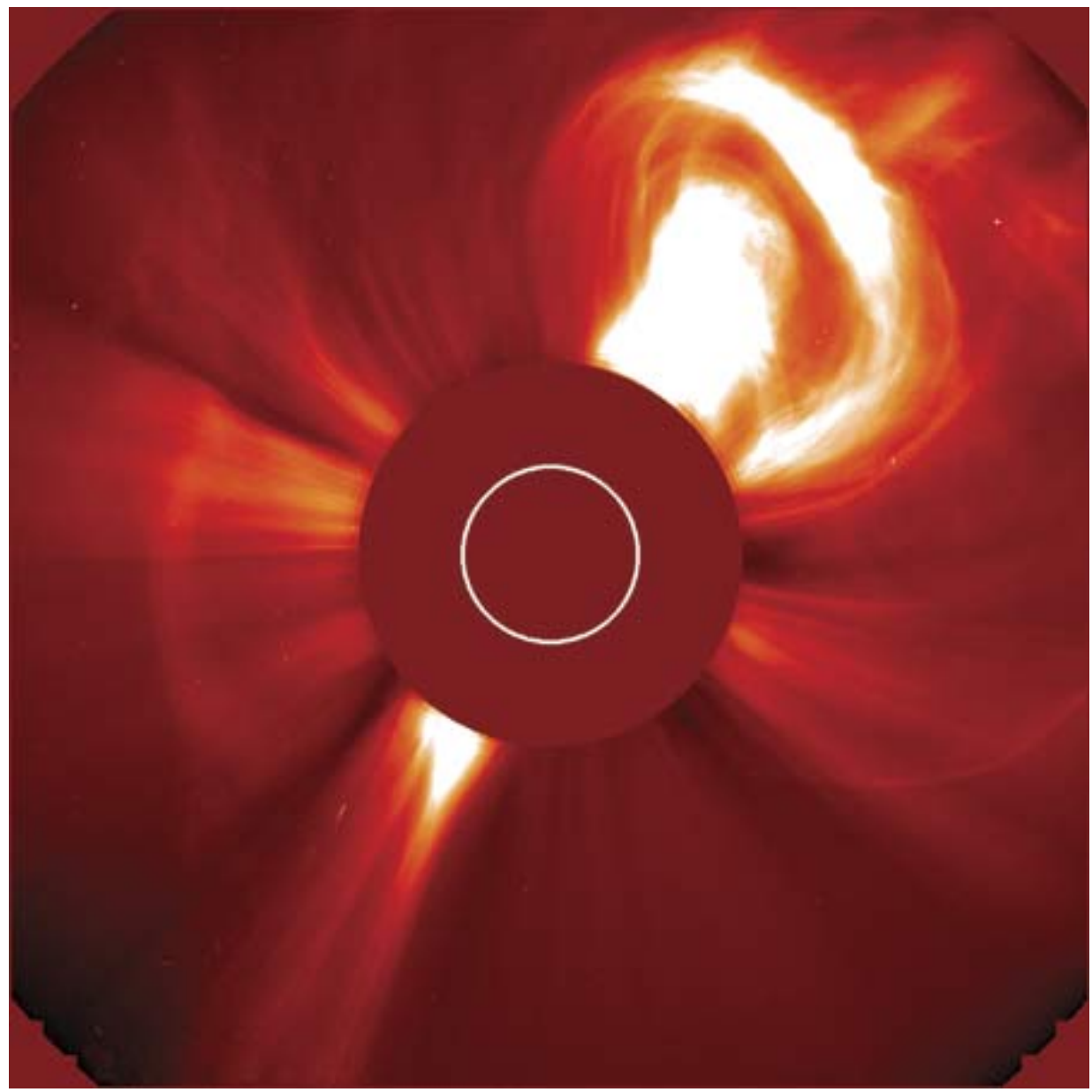

Figure 2.1.: SOHO/LASCO C2 image of a coronal mass ejection observed on December 2002. The three part structure of the CME is clearly visible in this event, and the Sun is behind the coronagraph occulter. Image courtesy of NASA 
2010). A statistical analysis performed by Michalek \& Yashiro (2013) on 694 CMEs showed that faster CMEs originate from more complex active regions, and that longer lived active regions are more likely to eject CMEs due to their complex magnetic field. Coronal mass ejections were first observed in the 1970s with the advent of space-based missions. The classic definition describes them as "an observable change in coronal structure that occurs on a time scale of a few minutes to several hours and involves the appearance (and outward motion) ${ }^{1}$ of a new, discrete, bright, white light feature in the coronagraph field of view" (Hundhausen et al., 1984). The brightness profile of CMEs is the result of photospheric light Thomson-scattered off electrons (see e.g. Webb \& Howard 2012). Brighter regions correspond to enhanced electron density. Since the corona is optically thin, white-light images of CMEs show the electron density integrated along the line of sight. For this reason the three-dimensional structure of CMEs is hard to infer. This difficulty has been alleviated with the launch of the NASA Solar TErrestrial RElations Observatory (STEREO, Kaiser et al. 2008) mission, which provides simultaneous multipoint observations of the inner heliosphere (also see Chapter 4).

From a morphological point of view coronal mass ejections are characterized by a threepart structure in which a bright leading edge is followed by a dark cavity and a bright core. The leading-edge is made of coronal material being swept up by the outflow, the dark cavity is interpreted as corresponding to a magnetically closed region, whereas the bright core is the outflowing prominence material (Chen, 2011). Observations of coronal mass ejections, however, have shown that only $\sim 30 \%$ of CMEs exhibit this three-part structure. In many cases the discrepancy can be attributed to viewing angle effects (Cremades \& Bothmer, 2004). Some of the events that do not conform to the three-part structure, on the other hand, do not present a bright core. This could be due to the fact that the filament material fell back on the solar surface, or that the erupting system is not associated to a filament at all (Gopalswamy, 2010).

The observed angular width of coronal mass ejections, i.e. their extent in terms of position angle, varies between $2^{\circ}$ and $360^{\circ}$, with an average around $41^{\circ}$ (see, e.g., Chen 2011, Gopalswamy 2010, Webb \& Howard 2012 and references therein). It is important to note that these values refer to geometries projected on the plane of the sky. CMEs with an apparent angular width of $360^{\circ}$ are referred to as halo CMEs, and are CMEs propagating in the direction of or away from the observer.

The speeds of CMEs range from $\sim 20$ to over $3000 \mathrm{~km} \mathrm{~s}^{-1}$ (Gopalswamy, 2010, Yashiro et al., 2004), with an average of roughly $300 \mathrm{~km} \mathrm{~s}^{-1}$ near solar minimum to about $500 \mathrm{~km} \mathrm{~s}^{-1}$ around solar maximum. Estimates of their mass yield values in the range of $10^{11} \mathrm{~kg}$ to $>10^{13} \mathrm{~kg}$, averaging at $10^{12} \mathrm{~kg}$ (Vourlidas et al., 2002). Their energy is between $10^{22}$ and $10^{25} \mathrm{~J}$, similar to the energy of solar flares. It has to be emphasized that these values are affected by projection effects and hence constitute lower limits. It has been found that wider CMEs tend to be faster and more massive than more narrow CMEs and consequently they carry more energy into interplanetary space (Gopalswamy, 2010, Gopalswamy et al., 2009).

The occurrence rate of coronal mass ejections is observed to track the solar cycle as well

\footnotetext{
${ }^{1}$ Schwenn (1996)
} 
as the sunspot number (Bosman et al., 2012), with 0.5 eruptions per day on average around solar minimum and 6 eruptions per day around solar maximum. Occasionally, even 10 eruptions in a single day have been observed (Gopalswamy et al., 2003a).
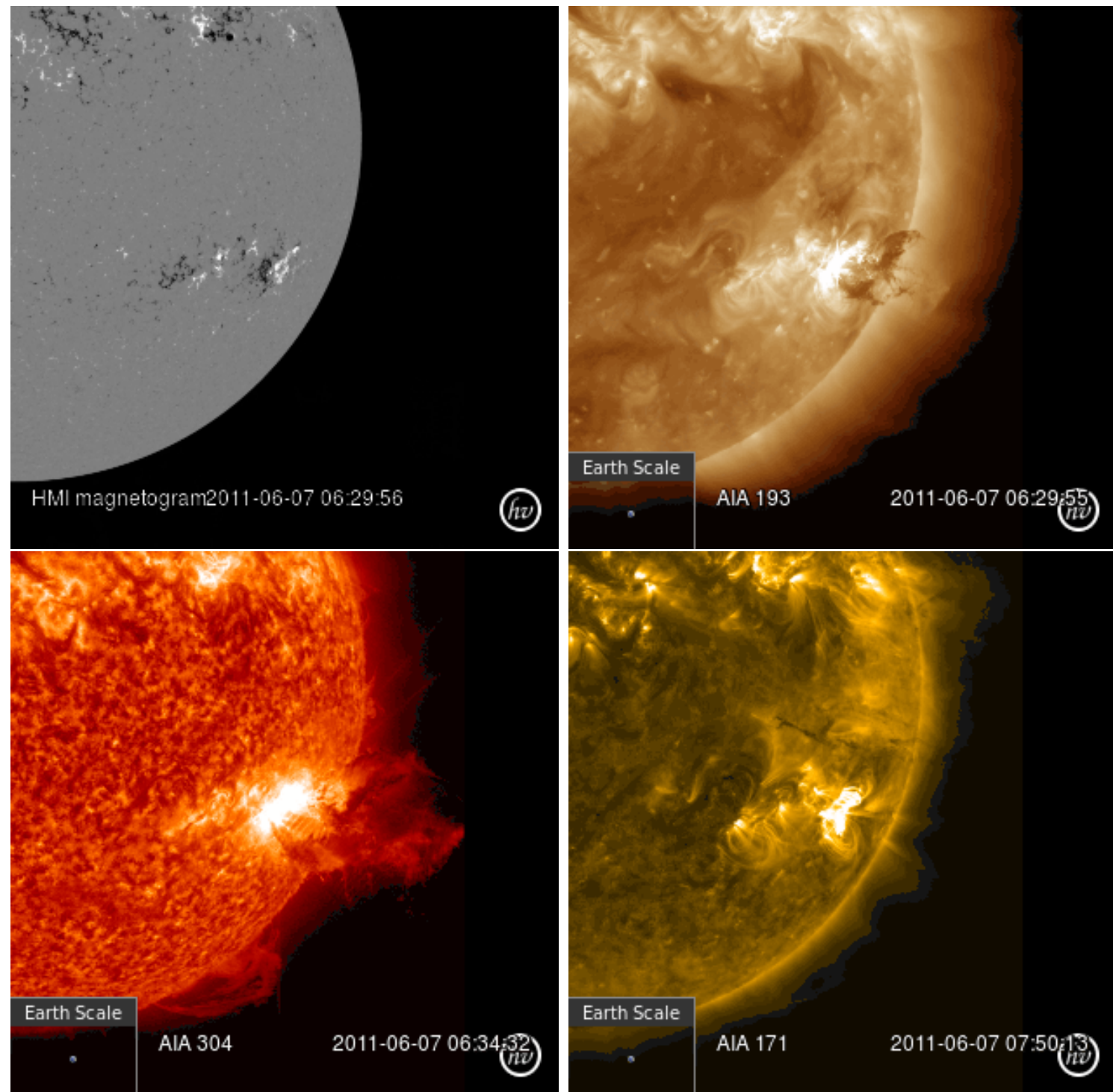

Figure 2.2.: SDO observations of the solar signatures of a CME, which erupted on 07 June 2011 from the SW quadrant of the solar disk. Top left: HMI magnetogram showing the magnetic field polarities and the CME source region. Top right: AIA EUV image at $193 \AA$ showing a flare and signatures of a prominence eruption associated with the CME. Bottom left: AIA EUV image at $304 \AA$ showing the prominence eruption. Bottom right: AIA EUV image at $171 \AA$ showing the post eruptive arcades. Images courtesy of NASA SDO consortium 


\subsection{Solar signatures of CME eruption}

Although the exact mechanisms behind the eruption of CMEs are not yet understood, it is clear that the driving forces are of magnetic origin (Chen, 2011). The source region of CMEs can be determined by combining coronagraph images with magnetograms and EUV observations of the solar surface. This way temporal and spatial correlations can be drawn between the eruption of CMEs and a series of accompanying processes that occur at the Sun around the time of CME onset (Cremades \& Bothmer, 2004). Coronal mass ejections are often found in association with flares, prominence eruptions, EUV dimmings, propagating waves, and post eruptive arcades (Webb \& Howard, 2012). This section will briefly describe the properties of these phenomena.

Solar flares are explosive phenomena occurring as a consequence of magnetic reconnection. They release energies in the range between $10^{28} \mathrm{erg}$ and $10^{32} \mathrm{erg}$ on timescales between $10^{3} s$ and $10^{4} s$. Solar flares are observed in X-rays, EUV, as well as radio and visible wavelengths (Benz, 2008). They are classified according to the peak of X-ray emission (see Table 2.1). As a matter of fact, the current understanding is that CMEs and flares are the product of the same physical process, i.e. reconnection at the Sun (see, e.g., Chen 2011, Webb \& Howard 2012 and references therein). Although there is no one-to-one association between CME and flares, these two phenomena are often observed together, especially in the most dramatic cases. Yashiro et al. (2005) showed that the probability of CME and flares association increases with the intensity of the flare, with a $100 \%$ probability for flares of class X3 and above. In the same study it was also found that faster and wider CMEs are associated with stronger flares.

EUV dimmings are localized decreases in the EUV brightness due to a decrease in the emission measure from the coronal region corresponding to the location of $\mathrm{CME}$ eruption (Webb \& Howard, 2012). The emission measure decreases as a consequence of the decrease in coronal density associated to the expulsion of plasma during the CME eruption.

Propagating EUV waves were first observed by SOHO/EIT, and for this reason they are often referred to as EIT waves (Thompson et al., 1998). Several models have been proposed, suggesting, for example, that EIT waves are fast mode MHD coronal waves (see, e.g. Wang 2000), slow mode waves (Wills-Davey et al., 2007), or apparently propagating density enhancements resulting from consecutive stretching of magnetic field lines above the erupting structures (see, e.g. Chen et al. 2005). Studies of CME and EUV waves association have shown that not every CMEs is associated with a coronal

\begin{tabular}{|c|c|}
\hline Class & Max X-ray flux $\left(\mathrm{erg} \cdot \mathrm{cm}^{-2} \cdot \mathrm{s}\right)$ \\
\hline $\mathrm{A} n$ & $n \cdot 10^{-8}$ \\
$\mathrm{~B} n$ & $n \cdot 10^{-7}$ \\
$\mathrm{C} n$ & $n \cdot 10^{-6}$ \\
$\mathrm{M} n$ & $n \cdot 10^{-5}$ \\
$\mathrm{X} n$ & $n \cdot 10^{-4}$ \\
\hline
\end{tabular}

Table 2.1.: Classification of solar flares according to the peak of associated X-ray emission. From https://www. unc.edu/ rowlett/units/scales/solar_flares.htm 
wave (also see Webb \& Howard 2012 and Chen 2011). A general consensus regarding the origin and nature of EUV waves has not yet been reached.

Another phenomenon observed in association to the CME eruption is the brightening of S-shaped or inverse S-shaped loop systems observed in X-ray, called post eruptive arcades or post eruptive loops. Tripathi et al. (2004) studied a set of over 230 post eruptive arcades between 1997 and 2000, and found a nearly-one-to-one relationship between their appearance and the eruption of coronal mass ejections. They concluded that these loop systems are a consequence of the rearrangement of the magnetic field due to the expulsion of a CME.

The presence of such accompanying processes on the solar surface allows the identification of the solar source region of CMEs via a combination of white-light images, EUV observations, and magnetograms.

The next section will provide an overview of the models that try to explain how the eruption of CMEs is triggered.

\subsection{Models for CME eruption}

Since the mechanisms behind the eruption of CMEs have not been yet fully understood and direct observations of such processes are not available, CME-associated phenomena on the Sun must be used as a proxy for the development and testing of models of CME eruptions (Chen, 2011). Beside the phenomena described in the previous sections, several other processes can occur before the onset of a CME. These precursors include, among others, radio noise storms, type III radio bursts groups, filament darkening and brightening, and SXR brightenings (Chen, 2011). Models for the triggering mechanisms of CME eruptions should be able to explain such processes, as well as to account for the necessary energy to propel the coronal material. It can be shown (Forbes, 2000) that especially for the more energetic CME events the only source that can provide sufficient energy is the magnetic field. The general picture is as follows: a flux rope, possibly holding a prominence, is maintained in equilibrium by a strapping field tied to the solar surface. After loss of equilibrium due to some triggering mechanism whose details are not yet fully understood, the flux rope starts rising and the strapping magnetic field is stretched. Antiparallel magnetic field lines start approaching in the wake of the erupting flux rope as it extends outwards, eventually leading to reconnection. This in turn results in a flare and the expulsion of the flux rope.

Several theories for the onset of the instability have been developed. In the remainder of the section we will briefly describe some of the models for CME initiation. The reader can refer to the review by Chen (2011) for a deeper discussion on such models.

\subsubsection{Tether cutting mechanism}

This model was proposed by Moore \& Labonte (1980) and is schematically illustrated in Figure 2.3. In this picture a filament is surrounded by a strongly sheared magnetic field, which is in turn held down by a less sheared magnetic field. As the shear increases the end points of the field lines $\mathrm{A}$ and $\mathrm{C}$, of opposite polarities, approach each other, 


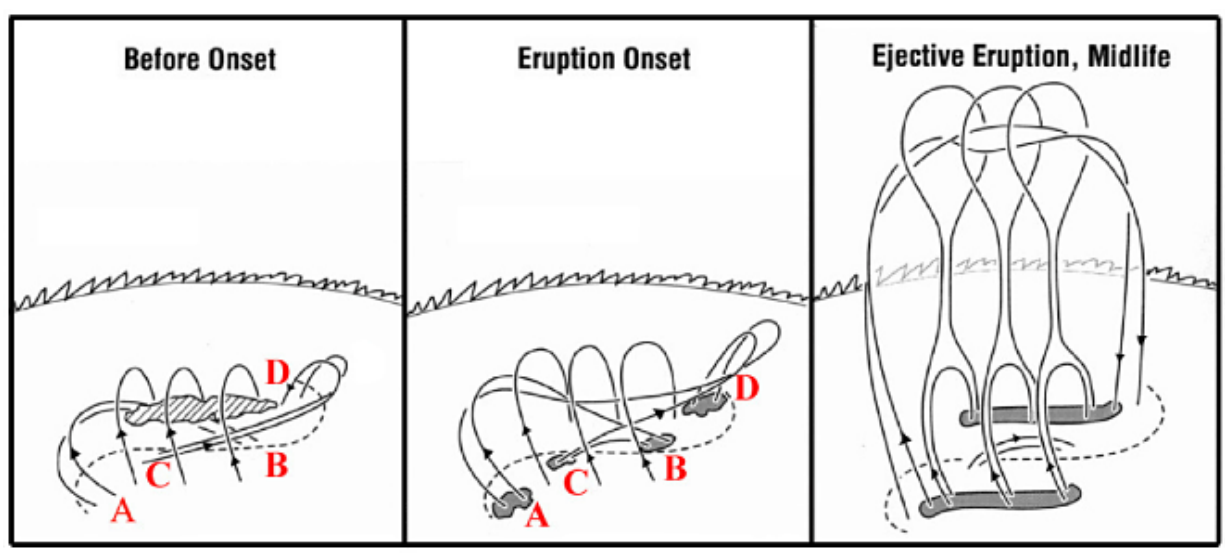

Figure 2.3.: Graphical representation of the tether cutting mechanism. From Chen (2011), adapted from Moore \& Labonte (1980).

and eventually reconnection occurs. As a consequence of reconnection the loop AD expands outward and the loop CB shrinks down. As the loop AD stretches up it pulls the filament and a current sheet is formed above the neutral line, in a configuration which is compatible with the CSHKP model (Carmichael, 1964, Hirayama, 1974, Kopp \& Pneuman, 1976, Sturrock, 1966). Reconnection occurs in the current sheet, with the consequent eruption of the CME and flares near the low corona. The model does not provide any explanation of how the sheared magnetic field forms. Shearing motions around the neutral line would be a source of such a configuration.

\subsubsection{Magnetic breakout model}

In this model, introduced by Antiochos et al. (1999), the magnetic field has a quadrupolar configuration in which a $\mathrm{X}$ type null point is formed above the central magnetic system (see Figure 2.4). Shear motions on the central part of the flux force it to expand and a current layer is formed at the location of the $\mathrm{X}$ null point. Reconnection processes in the current layer modify the topology of the magnetic field which was holding the core field down. As the latter starts rising a current sheet forms below it. Reconnection in the current sheet leads to eruption of a flux rope.

\subsubsection{Emerging flux triggering mechanism}

This model, proposed by Chen \& Shibata (2000), is based on the observational evidence that, in some cases, prior to CME eruptions, magnetic flux emerges with a configuration that is favourable for reconnection with the pre-existing coronal magnetic field (Feynman \& Martin, 1995). Figure 2.5 shows two different possible scenarios: in one case the emerging flux rises inside the filament channel, reconnecting with the smaller loops underneath the flux rope. This causes a local decrease in magnetic pressure and consequently plasma and magnetic fields on either side of the neutral line converge towards each other. In the other case the emerging flux rises outside the filament channel, and reconnects with the large-scale strapping magnetic field. In this new configuration 


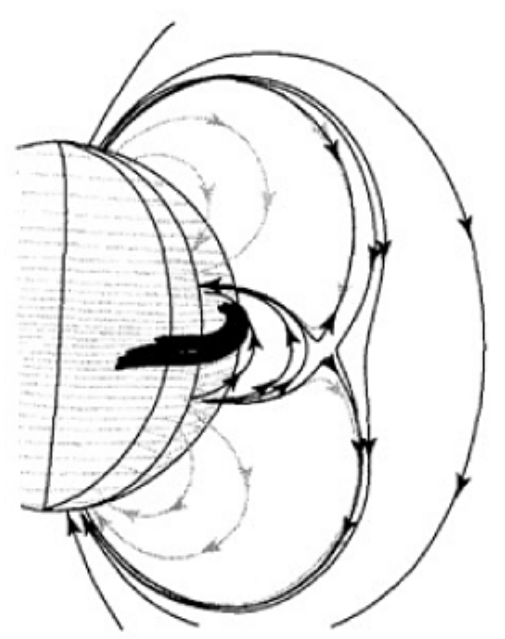

(a)

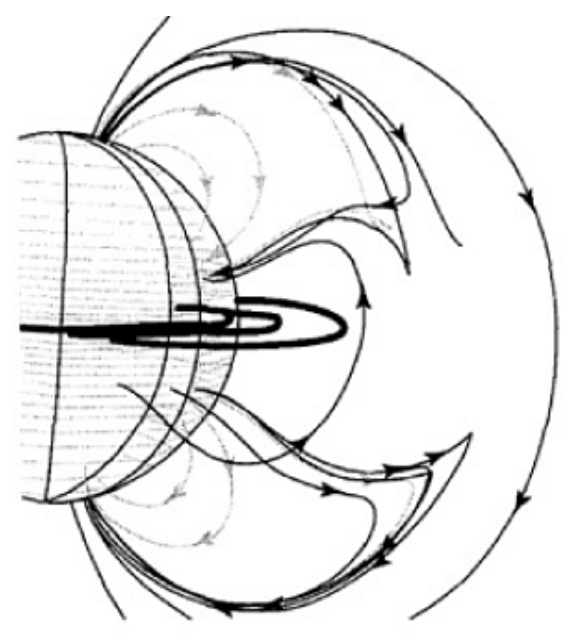

(b)

Figure 2.4.: Graphical representation of the magnetic breakout model. From Chen (2011), adapted from Antiochos et al. (1999).

the magnetic loop and the flux rope are pushed out. Both scenarios produce a magnetic field which is arranged in a way compatible to the CSHKP model. Reconnection in the current sheet produces flares, and the flux rope is accelerated by the Lorentz force.

\subsubsection{Instability and catastrophe-related triggering mechanisms}

This set of models is based on the hypothesis that the coronal magnetic field, under the action of photospheric motions and newly emerging flux, can evolve into a state of unstable equilibrium or away from equilibrium. Ideal MHD instabilities, such as the kink (Sakurai, 1976) or the torus instabilities (Bateman, 1978, Chen, 1989), would then trigger the eruption of a CME. Catastrophe related models (Priest \& Forbes, 1990), on the other hand, investigates MHD instabilities that develop in the non-linear regime.

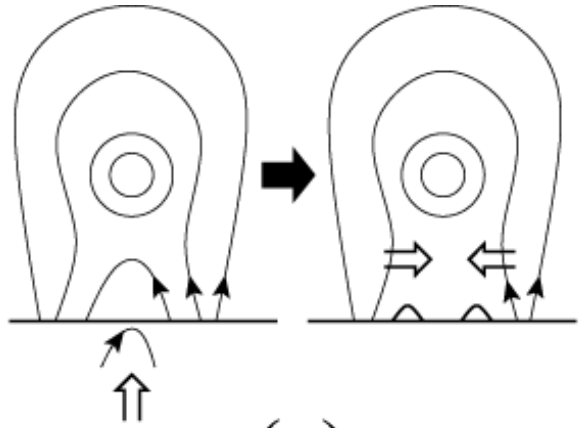

(a)

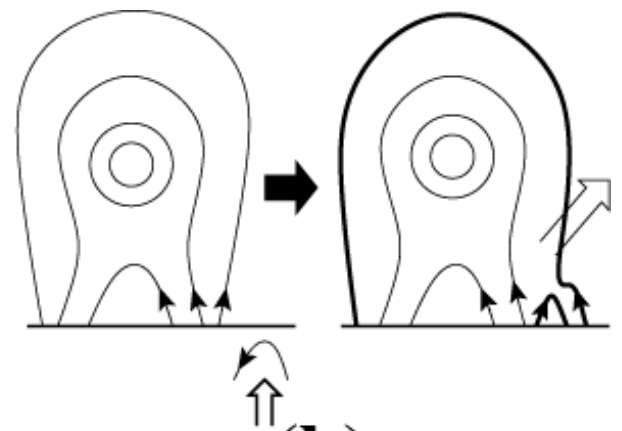

(b)

Figure 2.5.: Graphical representation of the emerging flux triggering mechanism. From Chen (2011), adapted from Chen \& Shibata (2000). 


\subsection{CME propagation}

The kinematic evolution of coronal mass ejections, according to a study by Zhang et al. (2001, 2004), can be divided into three phases: the initiation phase, lasting some tens of minutes, during which the loop starts rising with velocities of about $80 \mathrm{~km} \mathrm{~s}^{-1}$, the impulsive acceleration phase, when the erupting structure is quickly accelerated over timescales ranging from a few to some tens of minutes, and finally the propagation phase, over which the CME evolves at roughly constant velocity.

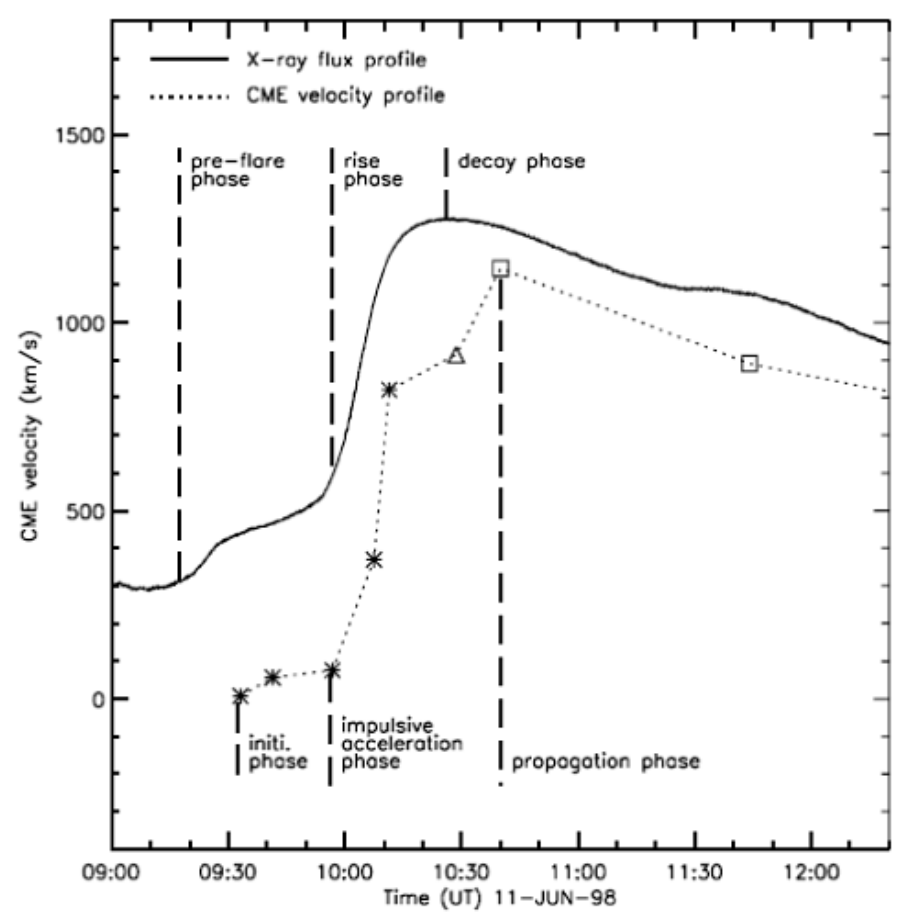

Figure 2.6.: Time profile of the soft X-ray flux of a flare (solid line) and the velocity of the associated CME (dotted line), showing the 3-phase initiation scenario proposed by Zhang et al. (2001, 2004). Image from Zhang et al. (2001). (C)AAS. Reproduced with permission.

This three-phase scenario of CME initiation follows closely the light curve of the associated flare: the initiation phase is observed to occur before the onset of the X-ray flare, the impulsive acceleration phase is simultaneous to the flare rise phase, and finally the propagation phase starts after the peak of soft X-ray flares (see Figure 2.6). Subsequent statistical studies by Zhang \& Dere (2006) showed that during the impulsive phase the acceleration varies between some $10^{-3} \mathrm{~km} \mathrm{~s}^{-2}$ to over $4 \mathrm{~km} \mathrm{~s}^{-2}$, with an average of $0.331 \mathrm{~km} \mathrm{~s}^{-2}$. This phase can last between several to more than 1000 minutes, and it has been shown (see, e.g. Chen 2011) that the bulk of the strong acceleration takes place below $2 R_{\odot}$. In this work these early phases of CME evolution will not be taken into account. The analysis will instead be focused of the propagation phase, i.e. on the characterization of the CME evolution in the inner heliosphere. Observations of CMEs near the Sun, i.e. in the field of view of coronagraphs, show that their (projected) speeds vary by roughly a factor of 10 . On the other hand, for the events 
observed in-situ , the travel speeds vary by no more than a factor of 3 (Schwenn et al., 2005). According to observations, CMEs slower than the solar wind are accelerated, and CMEs faster than the solar wind are decelerated, so that their speed approaches the solar wind speed. Gopalswamy et al. (2000) found an empirical formula relating the mean acceleration of the CME to its measured speed in a coronagraph field of view:

$$
a\left[\mathrm{~ms}^{-2}\right]=1.41-0.0035 * v\left[\mathrm{~km} \mathrm{~s}^{-1}\right] .
$$

Zhang \& Dere (2006) found that the average acceleration in the propagation phase is $0.9 \mathrm{~m} \mathrm{~s}^{-2}$, and varies between -151 and $52 \mathrm{~m} \mathrm{~s}^{-2}$. As of today a full understanding of the interplanetary evolution of CMEs and their interaction with the ambient solar wind has not been attained. Some models invoke aerodynamic drag as a cause for the residual acceleration of CMEs during the propagation phase (Cargill, 2004, Tappin, 2006). Based on the fact that CMEs are low- $\beta$ systems propagating in the high- $\beta$ solar wind, these models assume that the CME evolution is only determined by plasma and fluid dynamics, and that the latter always evolves in the direction of reaching kinematic equilibrium with the solar wind. Other models take into account the shock dynamics to describe the evolution of the CME, since any significant interaction with the solar wind happens via the CME-driven shock (Dryer \& Smart, 1984, Smith \& Dryer, 1990). Finally, a different set of models treats the CME as ejecta expelled into the solar wind (Chen, 1996, Odstrcil et al., 2003). A review of these models is provided in the book "Coronal Mass Ejections. An Introduction" by Howard (2011).

\subsection{CME and shocks}

CMEs propagating with velocities higher than the local Alfvén speed can drive shocks ahead of themselves. In-situ measurements of the solar wind reveal the passage of interplanetary (IP) shocks, corresponding to sudden increase in the plasma and magnetic field parameters (Sheeley et al., 1985). While in earlier times it was believed that flares were the cause of interplanetary shocks at Earth, it is now well known that these shocks are driven by fast CMEs (Gosling, 1993b, Sheeley et al., 1985). Given the Alfvén speed profile in the corona and the CME speeds at those heights, it was thought for a long time that CMEs could drive shocks already at those locations (Mann et al., 2003). Indirect signatures of the presence of shocks in white-light images were found already in the 1970s (Hildner et al., 1975), and bright forerunners ahead of CMEs were interpreted as white-light signatures of compressed plasma downstream of the CME-driven shock in the late 1980s (Sime \& Hundhausen, 1987). The first direct, confirmed detection of a CME-driven shock was obtained by Vourlidas et al. (2003). By means of remote-sensing observations and MHD simulations, they showed that the observed enhanced density feature preceding the CME in the coronagraph images was the consequence of a CME-driven shock. Despite the fact that many fast CMEs have been observed throughout the last decades, detection of shocks in white-light observations is not quite as frequent, an issue that has been addressed by Vourlidas (2006). The author introduced a criterion for the detectability of shocks in white-light images. 
In particular the shock strength X, i.e. the ratio between downstream and upstream densities, should satisfy the following condition:

$$
X \geq \frac{3 \sigma}{d \rho_{u}}+1
$$

where $\sigma$ is the background signal in coronagraph or (heliospheric imagers) observations, $d$ is the thickness of the shock along the line of sight, and $\rho_{u}$ is the upstream coronal density. The visibility curves derived for shocks of different thickness show that, as expected, a larger shock strength is required in order to detect the shock at higher heliocentric distances. For higher density compression the contrast at the shock front in remote-sensing observations is enhanced and the shock is more clearly detectable. In the same work the author also introduces two types of shocks, based on their appearance in coronagraph observations. The first type exhibits what, in analogy to the standing terrestrial shock, is referred to as bow shock morphology. The second type is classified as double front shock, due to the presence of two brightness features in the images: a forerunner with a sharp boundary followed by a filament-like structure. The former is attributed to the CME-driven shock, while the latter is believed to be a consequence of plasma pile-up at the flux rope leading-edge. Examples of shocks exhibiting the two different morphological characteristic are shown in Figure 2.7. Ontiveros \& Vourlidas (2009) analysed a set of fast $\left(v \geq 1500 \mathrm{~km} \mathrm{~s}^{-1}\right)$ CMEs observed by LASCO during the period from 1997 to 1999. They found that in $86 \%$ of the cases a shock could be detected in the images. The density jump across the shock was computed, and a correlation was found between the CME kinetic energy and the density jump across the shock, as well as between the CME momentum and the density compression. No correlation seemed to arise between the CME speed and the compression ratio. The compression ratio estimates were found to be in agreement with their values in-situ. Their results confirmed the hypothesis that CME-driven shocks can be detected in white-light images and that their properties can be inferred based on such observations. However, when considering the correlations found by Ontiveros \& Vourlidas (2009) one should take into account the fact that velocity estimates based on single viewpoint observations are heavily hindered by projection effects (Vourlidas et al., 2000). Mass estimates, moreover, are also subject to large errors due to projection effects and the need to assume a specific 3D geometry for the CME. Multipoint observations like the ones used in the present work heavily reduce the errors associated with projection effects. The works by Ontiveros \& Vourlidas (2009), Vourlidas (2006), Vourlidas et al. (2003) paved the way for several studies in which the shock parameters and the shock kinematic evolution were determined from remote-sensing observations (Gopalswamy \& Yashiro, 2011, Kim et al., 2012, Maloney \& Gallagher, 2011, Poomvises et al., 2012). The authors employed models which relate the standoff distance of the shock and the geometry of the driver to the density jump across the shock itself (Farris \& Russell, 1994, Seiff, 1962, Spreiter et al., 1966). Such models were employed in this work in combination with stereoscopic observations of fast CMEs, and will be illustrated in depth in Chapter 7. 

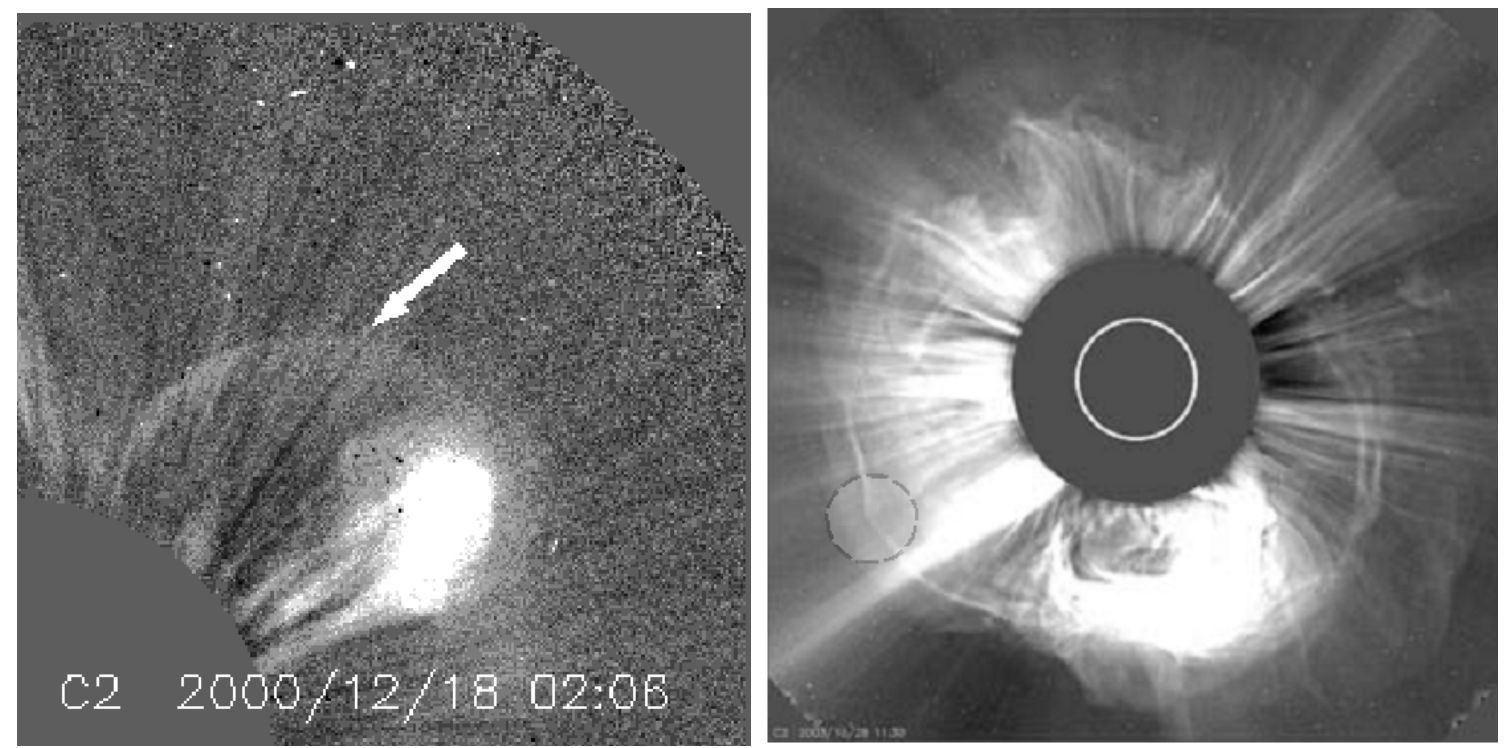

Figure 2.7.: Left panel:SOHO/LASCO C2 observations of a CME-driven shock conforming to the bow shock morphology. The arrow indicates the white-light signature of the bow shock. Adapted from Vourlidas et al. (2003). C AAS. Reproduced with permission.

Right panel: SOHO/LASCO C2 observations of a CME-driven shock conforming to the double front morphology. The circle indicates the double front shock. Adapted from Vourlidas (2006).

\subsubsection{Interplanetary consequences of CME-driven shocks}

CME-driven shocks are the main drivers of the acceleration of particles in the so-called Solar Energetic Particles (SEP) events measured in interplanetary space (Reames, 1999). These are sudden increases in the flux of energetic particles, and are subdivided in two categories: impulsive and gradual. Impulsive SEP events occur on time scales of a few hours, and are thought to be accelerated by flares. They exhibit large electron to proton and ${ }^{3} \mathrm{He}$ to ${ }^{4} \mathrm{He}$ ratios, as well as ionization states compatible with a source region temperature of $10^{7} \mathrm{~K}$. Gradual SEP events, on the other hand, occur on time scales of days, have high proton abundances, indicate a source region temperature of $10^{6} \mathrm{~K}$, and are thought to be accelerated at CME-driven shocks. These accelerate particles to the $\mathrm{MeV}$ energy range, whereas impulsive events are characterized by lower energies, in the keV range (see, e.g. Bothmer \& Daglis 2007, Ch. 3 and Kallenrode 2003). Several acceleration mechanisms have been proposed. In particular, for quasi-parallel shocks first-order Fermi acceleration is a good candidate. In this process particles are stochastically accelerated by multiple reflections upstream and downstream of the shock due to magnetic mirroring on turbulent magnetic fields. Gargaté et al. (2014), Kallenrode (2003). Park et al. (2012) showed that critical parameters which correlate with the flux and probability of gradual SEP events are the CME speed and angular width. The CME position is also an important factor, since magnetic connection to the Sun is achieved for west limb CMEs (Bothmer \& Daglis, 2007, Gopalswamy, 2005).

Accelerated energetic particles at shock fronts result also in emission at the radio fre- 


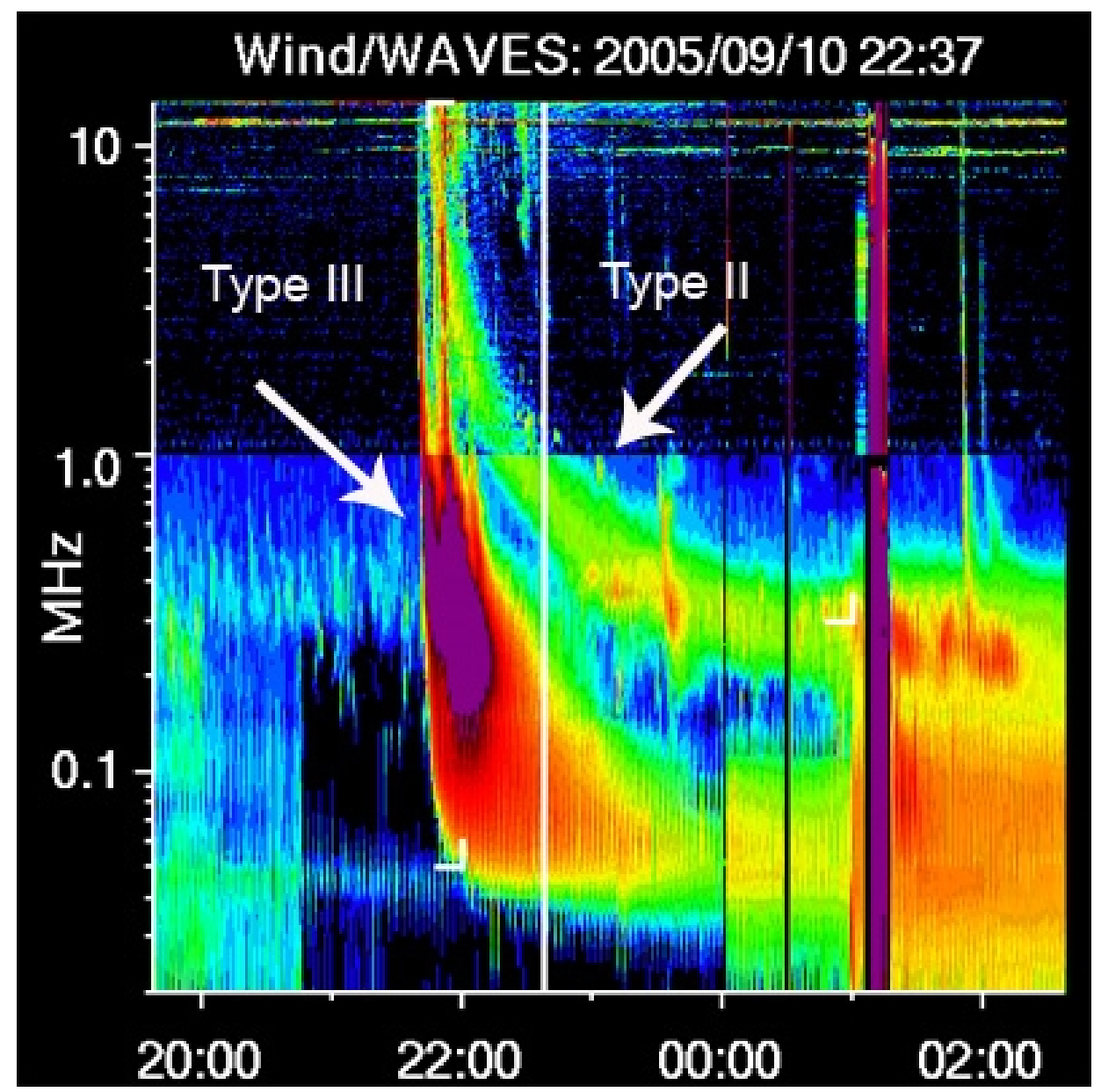

Figure 2.8.: Wind radio observations showing a type III and a type II radio burst, consequence of a fast CME which erupted at the Sun in September 2005. Image credit NASA/ESA.

quencies, the so-called radio bursts (Gopalswamy, 2005, 2011). Radio bursts result from the acceleration of energetic electrons, which emit electromagnetic radiation at the fundamental plasma frequency and its harmonics. This is defined in terms of the electron density: $\omega_{p}=\frac{n e}{m \epsilon_{0}}$. Because the electron density decreases further away from the Sun, so does the frequency of radio emission. It is therefore possible to determine at which locations such radiation was emitted. In particular, metric wavelengths corresponds to locations close to the Sun, whereas kilometric radiation corresponds to roughly 1 AU (Gopalswamy, 2005).

Radio type II bursts, first observed by Payne-Scott et al. (1947), are slowly drifting bursts caused by electrons accelerated by MHD shocks (Uchida, 1960, Wild \& McCready, 1950). The association with CME-driven shock was possible thanks to combined observations by in-situ spacecraft recording IP shocks, and later by white light observations of bright forerunners ahead of CME (Cane et al., 1987, Gopalswamy et al., 2001, Sheeley et al., 1985). Another class of radio bursts, referred to as type III bursts, is also detected. Radio type III bursts exhibit high frequency drift rates and appear as almost vertical tracks in dynamic spectra (see Figure 2.8). They are emitted 
by electron beams propagating along open magnetic field lines (see, e.g. Gopalswamy 2005 and references therein). Type III radio burst are thought to be associated to flares. Figure 2.8 shows radio measurements by the Wind spacecraft in which a radio type II burst and a radio type III burst can be identified. These are associated to the eruption and propagation of a fast CME.

\subsection{In-situ signatures}

Continuous monitoring of the solar wind parameters in the last decades has shown that three distinct types of solar wind exist: a fast solar wind component, with speeds ranging between 450 and $800 \mathrm{~km} \mathrm{~s}^{-1}$, a slow solar wind component, with speeds lower than $450 \mathrm{~km} \mathrm{~s}^{-1}$, and transient solar wind flows often times associated to interplanetary shocks (see, e.g Bothmer \& Daglis 2007, Ch. 3). The latter appear in the data as sudden enhancements in the solar wind speed, density, temperature, and magnetic field magnitude. The connection between interplanetary shocks at Earth and coronal mass ejections at the Sun, and the consequent identification of CMEs as the cause of interplanetary shocks Sheeley et al. (1985), was possible thanks to the combination of coronagraph and in-situ measurements. CMEs detected in-situ and in interplanetary space are often referred to as interplanetary CMEs (ICMEs).

The properties of the plasma in the sheath, the region behind the shock, are a result of compression and heating of the solar wind by the propagating CME. The magnetic field inside the sheath might be substantially distorted by draping of the interplanetary magnetic field (IMF) around the CME (Schwenn et al., 2005). A tangential discontinuity often separates the sheath and the CME material, although the boundaries of CMEs are often not easy to identify (Richardson et al., 2002, Schwenn et al., 2005). CMEs, in a fraction of the cases, are characterized by a smooth rotation of the magnetic field and lower plasma $\beta$ compared to the solar wind values. The low $\beta$ is a result of the enhanced magnetic field strength $(B>10 n T)$ and the reduced plasma temperature and densities inside the CME. This subset of CMEs is referred to as magnetic clouds (MCs, Burlaga et al. 1981), and present a magnetic topology compatible with the picture of a force-free cylindrical flux rope (see, e.g. Bothmer \& Schwenn 1998, Goldstein 1983). Other signatures include enhanced helium to proton ratios and anomalies in elemental composition and ionization states suggesting that the plasma originated from the corona (Cane \& Richardson, 2003). Lower SEP intensities are also detected, as the particles accelerated by the shocks cannot efficiently penetrate the magnetically closed region inside the CME. The presence of bidirectional particle flows is interpreted as an indication that part of the CME is magnetically connected to the Sun (Gosling, 1993b, Schwenn et al., 2005). The often observed declining velocity profile is compatible with an expanding structure whose leading-edge propagates at velocity $v_{\text {prop }}+v_{\text {exp }}$, and whose trailing edge propagates at velocity $v_{\text {prop }}-v_{\text {exp }}$, with a smooth transition between the two (Webb \& Howard, 2012). The quantities $v_{\text {prop }}$ and $v_{\text {exp }}$ are respectively the propagation and expansion speed of the CME. Furthermore, the magnetic field and plasma parameters often show a lower variance compared to the solar wind and the highly turbulent sheath region. As already mentioned, not all 


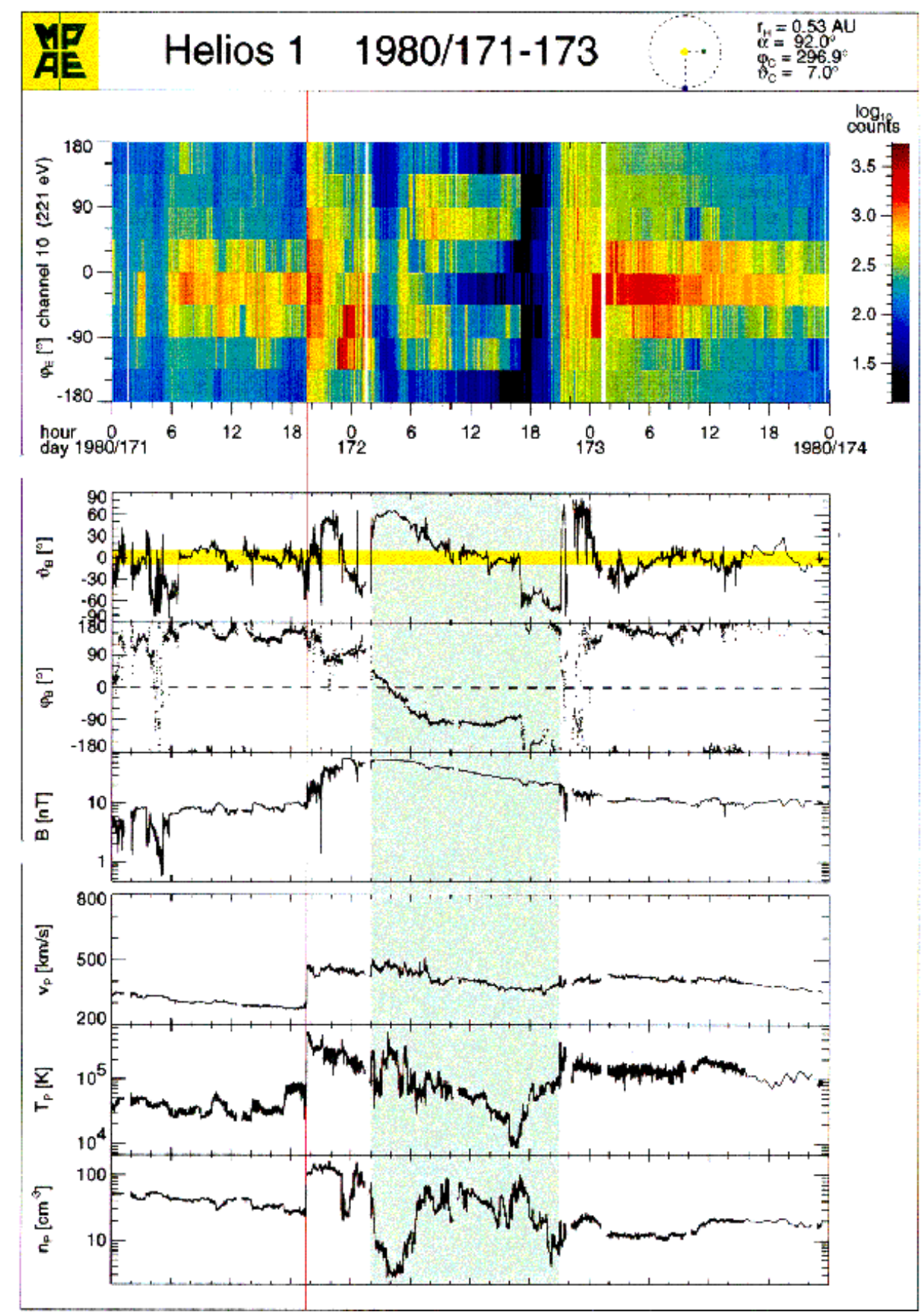

Figure 2.9.: Helios 1 in-situ measurements of the solar wind revealing a shock wave on 19 July 1980, followed by a CME with unique magnetic cloud signatures. In the upper panel bidirectional electron flows can be seen in measurements of the flow angle $\phi_{E}$ of suprathermal electrons. The value $\phi_{E}=0^{\circ}$ corresponds to propagation in the anti-Sunward direction. Negative values of the pitch angles correspond to clockwise direction, whereas positive values correspond to counter clockwise propagation. Reprinted from Bothmer (1999) with the permission of AIP publishing. 
CMEs exhibit magnetic cloud signatures, and not necessarily all of the properties listed above are present simultaneously in each event. Webb \& Hundhausen (1987) estimated that roughly 30\% of CMEs in the period between 1978 and 1982 were magnetic clouds. Bothmer \& Schwenn (1996) estimated the fraction to be 41\%, while Marubashi (2000) suggested that $80 \%$ of the in-situ CMEs are magnetic clouds, and that the lack of MC signatures is due to a non-central passage of the CME over the spacecraft. Of course it is also possible that the original structure did not possess a flux rope configuration at all. An example of an ICME presenting in-situ magnetic cloud signatures is provided in Figure 2.9.

In-situ observations of the solar wind, other than for the investigation of the physical properties of the different solar wind features, can be used for short-term space weather forecast purposes. They also provide an excellent tool for validation of models for the propagation of coronal mass ejections, since arrival time and speeds can be related and directly compared to observations. In this work we use Advanced Composition Explorer (ACE, Stone et al. 1998) data in order to verify arrival time and speed estimates for CMEs and shocks detected in remote-sensing observations, as well as the extrapolated shock parameters.

\subsection{Effects on Earth}

This section discusses the effects of CMEs and CME-driven shocks on the terrestrial environment. An in-depth review on the topic of space weather is provided in the book "Space Weather: Physics and Effects" by Bothmer \& Daglis (2007), which is the main source of the information presented here.

Impacts of solar activity on technology were already observed in the late 19th century, when disruption of the telegraph lines occurred in close association to auroras. A famous example is the Carrington event in 1859, when a very strong geomagnetic storm affected telegraph communication on a global scale. As technology advances and affects increasingly numerous aspects of everyday life, our society becomes more and more vulnerable to the effects of solar activity.

The initiation of geomagnetic storms is dependent on the presence of a southward component of the IMF. This allows reconnection with the northward magnetospheric field with subsequent injection of plasma in the nightside magnetosphere (Bothmer \& Daglis, 2007). Geomagnetic activity is a product of induction currents which, in turn, are produced by the electric field $\mathbf{E}=-\mathbf{v} \times \mathbf{B}$ imposed by the solar wind plasma and the IMF. The result is a system of currents whose major components are the ionospheric polar auroral electrojects and the near equatorial magnetospheric ring currents. The induction currents produced in geomagnetic storms have several effects, even at the ground. They result in the production of geomagnetic induced currents (GIC) which might interfere with the functioning of power transmission systems, oil and gas pipelines, telecommunication systems and railways. A very famous example is the nine hour-long blackout produced by GICs in the Hydro-Québec power system which occurred in March 1989.

Ionospheric conditions during geomagnetic storms can also affect the precision of Global 
Positioning System (GPS) locations. The radio signal transmitted from GPS satellites to the ground, in fact, travels through the ionosphere. Interaction between the radio signal and the ionospheric plasma leads to delays in the signal transmission, which in turn result in errors in the determined GPS positions. The delay, in first approximation, is proportional to the Total Electron Content (TEC), i.e. the electron density integrated along the line of sight. In normal conditions models have been developed which can compensate for the effects of the ionosphere. However, when geomagnetic storms occur the ionospheric conditions can change quite drastically, and the aforementioned models cannot provide accurate locations. For a more detailed discussion on the effects of space weather in satellite navigation the reader is referred to Bothmer \& Daglis (2007), Ch. 13.

As already discussed in this chapter, CME-driven shocks accelerate energetic particles to energies of the order of $\mathrm{MeV}$ and above. Such particles produce a hazardous environment for human space flights to the moon or to Mars, cause degradation to flying instruments, and can pose a threat to the health of passengers and crews on high latitude flights. near-Earth environments the reader can refer to Bothmer \& Daglis (2007). Although co-rotating interaction regions (CIRs), i.e. regions of transition between fast and slow solar wind streams, can trigger geomagnetic activity, all major geomagnetic storms are caused by CMEs. In particular Bothmer \& Schwenn (1995) showed that almost all geomagnetic storms with $K p>7+$ are driven by $\mathrm{CMEs}^{2}$, as they are associated to the largest values of the solar wind speed and the strongest southward magnetic field component at the position of Earth (also see Gosling 1993a, Richardson et al. 2002). Geomagnetic storms are triggered by CMEs in two different ways. On the one hand, the magnetic field inside CMEs can present an intrinsic southward component. On the other hand, especially for the fast events driving shocks, the draping of the IMF might produce a southward B component at Earth (Bothmer \& Schwenn, 1998). The severity of geomagnetic storms may be enhanced through the interaction of multiple CMEs (Bothmer, 1999, 2004, Gopalswamy et al., 2002).

The present chapter introduced coronal mass ejections and their driven shocks. It discussed their observational properties, models for their interplanetary propagation and their effects on Earth. Remote-sensing observations of CMEs and CME-driven shocks in the corona and inner heliosphere were used in this work to investigate their evolution and properties. Chapter 3 will provide an overview of the MHD equations and as well as of MHD waves, discontinuities, and shocks. The instruments providing the observations analysed in the present work will be described in Chapter 4. The employed models will be introduced in Chapters 5, 6 and 7. Chapter 8 will present the results which will

\footnotetext{
${ }^{2}$ The Kp index is a quasi-logarithmic parameter, ranging from 0 to 9 , used to indicate the strength of geomagnetic storms (Bothmer \& Daglis, 2007, Ch. 3).
} 
be summarized and discussed in Chapter 9. 

3. Basic Concepts of Magnetohydrodynamics 

This chapter will briefly describe the derivation of the equations of magnetohydrodynamics (MHD) in the limit of infinitely conductive fluids. The properties of MHD waves and subsequently those of MHD discontinuities and shocks will be discussed. The concepts introduced here will be useful for the understanding of the models presented in Chapter 7 The details of the derivations will be omitted, but the properties of MHD plasmas will be described. A full derivation of the equations presented in this Chapter is provided, e.g, by Baumjohann \&5 Treumann (1996).

\subsection{MHD equations}

Plasmas, on time scales larger that the ion gyrofrequency, and on spatial scales much larger than the ion gyroradius, are described by the equations of magnetohydrodynamics (MHD). These are derived as a simplification of the multi-fluid equations, which are in turn obtained by computing the moments of the Boltzmann equation. When treating plasma like a fluid, the main focus is on the evolution of the macroscopic properties of the system, rather than the on evolution of each single particle in the fluid. In dynamical systems the state of a particle at a given time $t$ can be represented as a point in phase space, i.e. the space with coordinates $\boldsymbol{x}$ and $\boldsymbol{v}$, position and velocity. It is possible to define a phase space density

$$
F(\boldsymbol{x}, \boldsymbol{v}, t)=\sum_{i} \delta\left(\boldsymbol{x}-\boldsymbol{x}_{\boldsymbol{i}}(\boldsymbol{t})\right) \delta\left(\boldsymbol{v}-\boldsymbol{v}_{\boldsymbol{i}}(\boldsymbol{t})\right),
$$

where the sum is taken over each particle in the system. If the system is composed of several species there will be a similar expression for each specie.

In principle it is possible to write the equation of motion for each single particle. Coupling them with the Maxwell equations yields a closed system of equations that can be integrated to derive the evolution of all the particles and the fields in the plasma. However, such an approach involves an extremely large number of degrees of freedom, and is not computationally tractable for any reasonably-sized system.

The first step to reduce the complexity of the system is to define an ensemble average of the phase space density, the distribution function $f(\boldsymbol{x}, \boldsymbol{v}, t)$, such that

$$
F(\boldsymbol{x}, \boldsymbol{v}, t)=f(\boldsymbol{x}, \boldsymbol{v}, t)+\delta F(\boldsymbol{x}, \boldsymbol{v}, t) .
$$

This means that the exact phase space density is described as an average, the distribution function, plus fluctuations. These have the property that $\langle\delta F(\boldsymbol{x}, \boldsymbol{v}, t)\rangle=0$. The distribution function can be thought as the probability at a given time $t$ to find a particle in a volume element $d \boldsymbol{x} d \boldsymbol{v}$ of the phase space. The evolution of the distribution function is described in terms of the Boltzmann equation. In the presence of a 
magnetic field, if the effects of gravity can be neglected, the Boltzmann equation ${ }^{1}$ can be written as:

$$
\frac{\partial f}{\partial t}+\boldsymbol{v} \cdot \boldsymbol{\nabla}_{\boldsymbol{x}} f+\frac{q}{m}(\boldsymbol{E}+\boldsymbol{v} \times \boldsymbol{B}) \cdot \boldsymbol{\nabla}_{\boldsymbol{v}} f=\left(\frac{\partial f}{\partial t}\right)_{c} .
$$

The term on the right hand side describes the time variation of the distribution function due to collisions. When this is set to zero the above equation yields the well known Vlasov equation. Although the complexity of the system has been reduced by the introduction of the distribution function, the latter does not represent a measurable quantity. Physical quantities like density, velocity and pressure can be derived by taking moments of the distribution function. The $i-t h$ order moment of $f(\boldsymbol{x}, \boldsymbol{v}, t)$ is defined as the integral

$$
M_{i}(\boldsymbol{x}, t)=\int f(\boldsymbol{x}, \boldsymbol{v}, t) \boldsymbol{v}^{i} d \boldsymbol{v}
$$

In particular, the particle density $n$ is the 0 -th order moment of the distribution function, the bulk velocity $v$ is the 1st order moment, and the pressure tensor $\boldsymbol{P}$ is related to the 2 nd order moment. The heat flux $\boldsymbol{q}$ is derived from the 3rd order moment. The evolution of the macroscopic properties of the plasma, i.e. its density, velocity, and pressure can now be derived without knowledge of the exact evolution of the distribution function. The plasma can be treated with the tools provided by fluid theory. This is done by taking moments of the Boltzmann equation for each species in the plasma, and yields a system of equations in the macroscopic velocity, density, pressure, and the electric and magnetic fields. Let's assume for the sake of simplicity that the plasma is composed of electrons and one specie of ions. Charge and current can be defined as

$$
\begin{aligned}
& \rho=e\left(n_{i}-n_{e}\right) \\
& \boldsymbol{j}=e\left(n_{i} \boldsymbol{v}_{\boldsymbol{i}}-n_{e} \boldsymbol{v}_{\boldsymbol{e}}\right)
\end{aligned}
$$

Introducing the fluid density, mass and velocity as

$$
\begin{aligned}
& n=\frac{m_{e} n_{e}+m_{i} n_{i}}{m_{e}+m_{i}} \\
& m=m_{e}+m_{i} \\
& \boldsymbol{v}=\frac{m_{e} n_{e} \boldsymbol{v}_{\boldsymbol{e}}+m_{i} n_{i} \boldsymbol{v}_{\boldsymbol{i}}}{m_{e} n_{e}+m_{i} n_{i}}
\end{aligned}
$$

it is possible to derive the MHD equations in the case of a one-fluid plasma:

\footnotetext{
${ }^{1}$ in SI units
} 


$$
\begin{aligned}
& \frac{\partial n}{\partial t}+\boldsymbol{\nabla} \cdot(n \boldsymbol{v})=0 \\
& \frac{\partial(n m \boldsymbol{v})}{\partial t}+\boldsymbol{\nabla} \cdot(n m \boldsymbol{v} \boldsymbol{v})=-\boldsymbol{\nabla} \cdot \boldsymbol{P}+\rho \boldsymbol{E}+\boldsymbol{j} \times \boldsymbol{B} \\
& \boldsymbol{E}+\boldsymbol{v} \times \boldsymbol{B}=\eta \boldsymbol{j}+\frac{1}{n e} \boldsymbol{j} \times \boldsymbol{B}-\frac{1}{n e} \boldsymbol{\nabla} \cdot \boldsymbol{P}_{\boldsymbol{e}}+\frac{m_{e}}{n e^{2}} \frac{\partial \boldsymbol{j}}{\partial t} \\
& \boldsymbol{\nabla} \times \boldsymbol{B}=\mu_{0} \boldsymbol{j}+\mu_{0} \epsilon_{0} \frac{\partial \boldsymbol{E}}{\partial t} \\
& \boldsymbol{\nabla} \times \boldsymbol{E}=-\frac{\partial \boldsymbol{B}}{\partial t} \\
& \boldsymbol{\nabla} \cdot \boldsymbol{B}=0 .
\end{aligned}
$$

They are respectively the mass conservation equation, the momentum equation, the generalized Ohm's law, Ampeŕe's law, Faraday's law and the soleinoidality condition for the magnetic field. The system has to be completed by an equation of state. Assuming quasi-neutrality and neglecting the displacement currents yields

$$
\begin{aligned}
& \frac{\partial n}{\partial t}+\boldsymbol{\nabla} \cdot(n \boldsymbol{v})=0 \\
& \frac{\partial n m \boldsymbol{v}}{\partial t}+\boldsymbol{\nabla} \cdot(n m \boldsymbol{v} \boldsymbol{v})=-\boldsymbol{\nabla} \cdot \boldsymbol{P}+\boldsymbol{j} \times \boldsymbol{B} \\
& \boldsymbol{E}+\boldsymbol{v} \times \boldsymbol{B}=\eta \boldsymbol{j}+\frac{1}{n e} \boldsymbol{j} \times \boldsymbol{B}-\frac{1}{n e} \boldsymbol{\nabla} \cdot \boldsymbol{P}_{\boldsymbol{e}}+\frac{m_{e}}{n e^{2}} \frac{\partial \boldsymbol{j}}{\partial t} \\
& \boldsymbol{\nabla} \times \boldsymbol{B}=\mu_{0} \boldsymbol{j} \\
& \boldsymbol{\nabla} \times \boldsymbol{E}=-\frac{\partial \boldsymbol{B}}{\partial t} \\
& \boldsymbol{\nabla} \cdot \boldsymbol{B}=0 .
\end{aligned}
$$

Moreover, using Equation 3.8d the so called Hall term $\boldsymbol{j} \times \boldsymbol{B}$ can be rewritten as

$$
\boldsymbol{j} \times \boldsymbol{B}=-\frac{1}{\mu_{0}} \boldsymbol{B} \times(\boldsymbol{\nabla} \times \boldsymbol{B}),
$$

which in turn can be expressed as

$$
\boldsymbol{j} \times \boldsymbol{B}=-\nabla \frac{B^{2}}{2 \mu_{0}}+\frac{1}{\mu_{0}} \nabla \cdot(\boldsymbol{B} \boldsymbol{B}) .
$$

The first term on the right hand side is the magnetic pressure, and the second term is the divergence of the magnetic stress tensor, encoding the tension and torsion introduced in the plasma by the magnetic field. The ideal MHD equations can be derived from the system of equations 3.8 by neglecting the Hall term, the time variation of the current $\boldsymbol{j}$ and the electron pressure $\boldsymbol{P}_{\boldsymbol{e}}$ and assuming infinite conductivity, i.e. taking $\eta=0$. Under these conditions Equation 3.8c reduces to $\boldsymbol{E}=-\boldsymbol{v} \times \boldsymbol{B}$, and the system of equations 3.8 can be written as: 


$$
\begin{aligned}
& \frac{\partial n}{\partial t}+\nabla \cdot(n \boldsymbol{v})=0 \\
& \frac{\partial n m \boldsymbol{v}}{\partial t}+\nabla \cdot(n m \boldsymbol{v} \boldsymbol{v})=-\boldsymbol{\nabla} \cdot\left(\boldsymbol{P}+\frac{B^{2}}{2 \mu_{0}} \boldsymbol{I}\right)+\frac{1}{\mu_{0}} \boldsymbol{\nabla} \cdot \boldsymbol{B} \boldsymbol{B} \\
& \frac{\partial \boldsymbol{B}}{\partial t}=\boldsymbol{\nabla} \times(\boldsymbol{v} \times \boldsymbol{B}) \\
& \boldsymbol{\nabla} \cdot \boldsymbol{B}=0 .
\end{aligned}
$$

Equation 3.11a is the continuity equation, expressing mass conservation. Equation $3.11 \mathrm{~b}$ is the momentum equation, describing the effects of the forces acting on the fluid. Equation 3.11c is the induction equation in the ideal MHD limit. It describes the temporal evolution of the magnetic field in a perfectly conducting fluid under the action of the velocity field of the fluid. Finally, Equation 3.11d is the soleinoidality condition for the magnetic field. It can be shown that it is already contained in Equation 3.11c. It implies that any change in the magnetic field strength cannot be local (see, e.g. Spruit 2013).

It is worth recalling that the MHD equations are valid if the fields and the fluid change on time scales much larger than those defined by the the ion gyrofrequency $\omega_{g}=\frac{|q| B}{m}$, and at scales larger than the ion gyroradius $r_{i}=\frac{m v_{\perp}}{|q| B}$.

\subsubsection{Frozen-in fields}

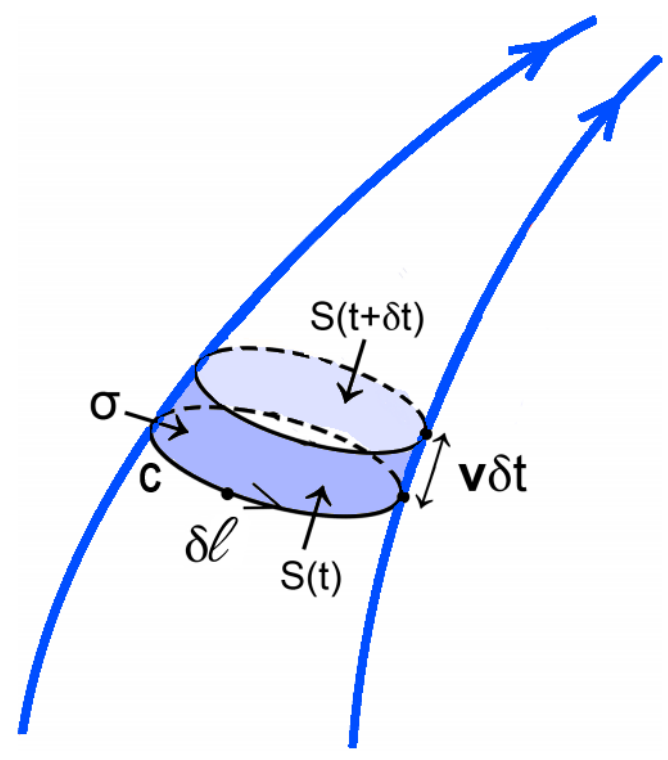

Figure 3.1.: Magnetic field lines through a surface $S$ enclosed by a curve $C$. From Spruit (2013) 
This section will show that for plasmas satisfying the ideal MHD equations the magnetic field is frozen-in the plasma, i.e. the magnetic flux is constant in time. The magnetic flux through an open surface $S$ enclosed by a curve $C$ and moving with the plasma is defined as

$$
\Phi=\iint_{S} \boldsymbol{B}(\boldsymbol{r}, t) \cdot \hat{\boldsymbol{n}} d S
$$

with $\hat{\boldsymbol{n}}$ normal to the surface. The temporal evolution of the magnetic flux is then due to two components: the time variation of the magnetic field at a fixed location and the deformation of the surface $S$ during the motion. The former is simply given by

$$
I_{1}=\iint_{S} \frac{\partial \boldsymbol{B}}{\partial t} \hat{\boldsymbol{n}} d S
$$

It can be shown (Spruit, 2013) that the latter can be expressed as

$$
I_{2}=\iint_{S(t+\delta t)} \boldsymbol{B}(\boldsymbol{r}, t+\delta t) \cdot \hat{\boldsymbol{n}} d S-\iint_{S(t)} \boldsymbol{B}(\boldsymbol{r}, t+\delta t) \cdot \hat{\boldsymbol{n}} d S .
$$

Furthermore, because $\boldsymbol{\nabla} \cdot \boldsymbol{B}=0$, the total flux through the volume enclosed by the surfaces $S(t), S(t+\delta t)$ and the side surface $\sigma$ (see Figure 3.1) is zero, and

$$
\iint_{S(t+\delta t)} \boldsymbol{B}(\boldsymbol{r}, t+\delta t) \cdot \hat{\boldsymbol{n}} d S-\iint_{S(t)} \boldsymbol{B}(\boldsymbol{r}, t+\delta t) \cdot \hat{\boldsymbol{n}} d S=\iint_{\sigma} \boldsymbol{B} \cdot \hat{\boldsymbol{n}} d S .
$$

The latter can be expressed as derived by considering the line element $\delta \boldsymbol{l}$ around the curve $C$ enclosing the surface $S$. The magnetic flux through the side surface $\delta \boldsymbol{\sigma}=$ $\boldsymbol{v} \delta t \times \delta \boldsymbol{l}$ swept out by $\delta \boldsymbol{l}$ during the time interval $\delta t$ is

$$
\begin{aligned}
\iint_{\sigma} \boldsymbol{B} \cdot \hat{\boldsymbol{n}} d S & =\oint_{C} \boldsymbol{B} \cdot(\delta \boldsymbol{l} \times \boldsymbol{v} \delta t) \\
& =\delta t \oint_{C}(\boldsymbol{B} \times \boldsymbol{v}) \cdot \delta \boldsymbol{l}
\end{aligned}
$$

so that

$$
\frac{d \Phi}{d t}=\iint_{S} \frac{\partial \boldsymbol{B}}{\partial t}+\oint_{C}(\boldsymbol{B} \times \boldsymbol{v}) \cdot d \boldsymbol{l}
$$

Using Stokes's theorem yields

$$
\oint_{C}(\boldsymbol{B} \times \boldsymbol{v}) \cdot d \boldsymbol{l}=\iint_{S} \boldsymbol{\nabla} \times(\boldsymbol{B} \times \boldsymbol{v}) \cdot \hat{\boldsymbol{n}} d S
$$

and finally, after rearranging

$$
\frac{d \Phi}{d t}=\iint_{S} \frac{\partial \boldsymbol{B}}{\partial t}-\nabla \times(\boldsymbol{v} \times \boldsymbol{B}) \cdot \hat{\boldsymbol{n}} d S
$$

For an infinitely conductive plasma the sum on the right hand side vanishes (see Equation 3.11c). In an ideal MHD plasma therefore magnetic field and plasma move together. A consequence of the frozen in theorem, moreover, is that if two plasma elements are associated to the same field line at a given instant in time, they will stay on the same field line at all times during the plasma motion. 


\subsection{MHD waves}

Disturbances in fluids propagate in the form of waves. The dispersion relation for propagating waves can be obtained by solving the linearised equations describing the dynamics of the fluid. For classical hydrodynamic fluids the propagating waves are sound waves, with speed $c_{s}^{2}=\frac{\partial p}{\partial n m}$. In MHD systems, on the other hand, three types of waves exist: Alfveń waves, fast magnetosonic waves and slow magnetosonic waves. When large disturbances are present the slow and fast waves steepen into shocks.

Alfven waves are non-dispersive, non-compressive ${ }^{2}$ waves that propagate along the magnetic field lines at the Alfven speed $v_{A}{ }^{2}=\frac{B^{2}}{\mu_{0} n m}$. They can be thought of as waves propagating along a tense string (the magnetic field line), in which the restoring force is the magnetic tension. The dispersion relation for Alfveń waves is

$$
\omega_{A}= \pm k \cos \theta v_{A},
$$

with $k$ wave number and $\theta$ angle between $k$ and the magnetic field.

The dispersion relation for magnetosonic waves, on the other hand, is given by:

$$
\omega_{m s}^{2}=\frac{k^{2}}{2}\left[\left(v_{A}^{2}+c_{s}^{2}\right) \pm \sqrt{\left(v_{A}^{2}+c_{s}^{2}\right)^{2}-4 v_{A}^{2} c_{s}^{2} \cos ^{2} \theta}\right]
$$

with $\theta$ angle between the magnetic field $\boldsymbol{B}$ and the wave vector $\boldsymbol{k}$. The properties of magnetosonic waves depend on two parameters only: the angle $\theta$ and the ratio of sound to Alfveń speed $\frac{c_{s}}{v_{A}}=\sqrt{\frac{\gamma \beta}{2}}$. The parameter $\beta$ is the ratio between gas and magnetic pressure, and $\gamma$ is the adiabatic index. The fast magnetosonic waves are those associated with the plus sign, and propagate at speed

$$
v_{m s, f}^{2}=\frac{1}{2}\left[v_{A}^{2}+c_{s}^{2}+\sqrt{\left(v_{A}^{2}+c_{s}^{2}\right)^{2}-4 v_{A}^{2} c_{s}^{2} \cos ^{2} \theta}\right],
$$

whereas the slow magnetosonic waves propagate at speed

$$
v_{m s, s}^{2}=\frac{1}{2}\left[v_{A}^{2}+c_{s}^{2}-\sqrt{\left(v_{A}^{2}+c_{s}^{2}\right)^{2}-4 v_{A}^{2} c_{s}^{2} \cos ^{2} \theta}\right] .
$$

Magnetosonic waves, similarly to the Alfvén wave, are non-dispersive, but the density and pressure fluctuations are non-zero. In the direction perpendicular to the magnetic field, i.e. $\theta=90^{\circ}$, the expression for the fast and slow magnetosonic speeds in Equation 3.22 reduces to

$$
v_{m s}^{2}=\frac{1}{2}\left[v_{A}^{2}+c_{s}^{2} \pm\left(v_{A}^{2}+c_{s}^{2}\right)\right] .
$$

Slow magnetosonic waves do not propagate, while the fast ones do isotropically at speed

$$
v_{m s \perp}^{2}=v_{A}^{2}+c_{s}^{2} .
$$

${ }^{2} \delta n m=0$ 


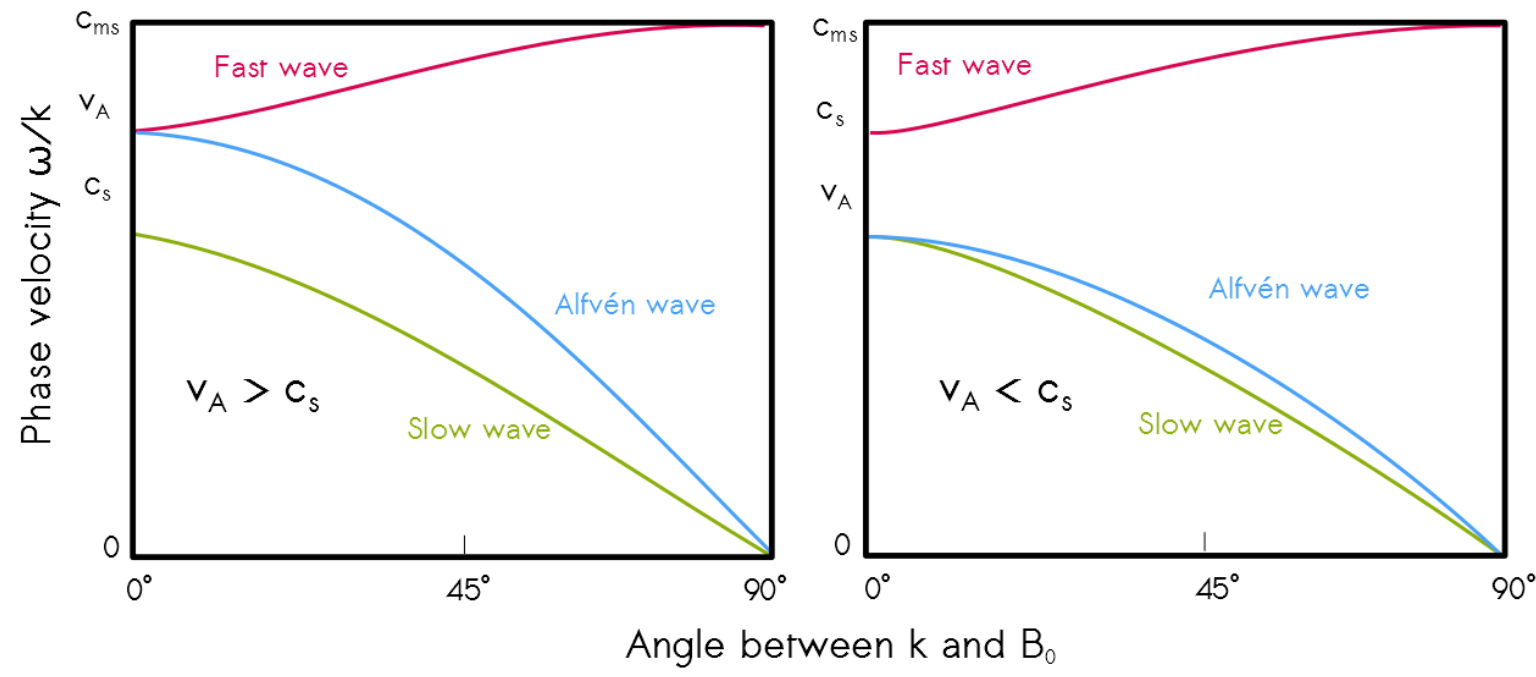

Figure 3.2.: Propagation speed of MHD waves as a function of the angle with respect to the direction of the magnetic field. The left panel shows the case in which the Alfven speed is larger than the sound speed, while the right panel illustrates the case in which the sound speed is larger than the Alfveń speed. Adapted from Baumjohann \& Treumann (1996).

In the direction parallel to the magnetic field, on the other hand, Equation 3.22 can be written as

$$
v_{m s / /}^{2}=\frac{1}{2}\left[v_{A}^{2}+c_{s}^{2} \pm\left(v_{A}^{2}-c_{s}^{2}\right)\right] .
$$

In the case $c_{s}<v_{A}$ the fast mode propagates with the Alfven speed and the slow mode consists of sound waves. If instead $c_{s}>v_{A}$ the fast mode propagates at the sound speed and the slow mode at the Alfven speed. The behaviour of MHD waves as function of the angle between the propagation direction and the magnetic field is illustrated in Figure 3.2, for the cases $c_{s}<v_{A}$ (left panel) and $c_{s}>v_{A}$ (right panel). The figures show that for $\theta=0^{\circ}$, i.e. in the direction parallel to the magnetic field, if $c_{s}<v_{A}$ $\left(c_{s}>v_{A}\right)$ the slow wave propagates at the sound (Alfveń) speed, and the fast wave propagates at the Alfven speed. As $\theta$ increases the speed of the fast wave increases over the Alfven (sound) speed, and three propagating wave modes are present. When $\theta$ reaches $90^{\circ}$, the fast mode propagates at speed $v_{m s \perp}^{2}=v_{A}^{2}+c_{s}^{2}$, while the slow and the Alfveń waves are suppressed.

\subsection{MHD discontinuities and shocks}

Whenever two plasmas that possess different properties interact, discontinuities arise. These are thin regions, i.e. regions smaller compared to the fluid length scales, but still larger than the Debye length and the ion gyroradius. In the context of the solar wind such discontinuities arise from its interaction with, e.g., planetary magnetospheres or propagating CMEs. In order to derive the jump conditions at a discontinuity, the one 
fluid ideal MHD equations (3.11) are considered. For a thin layer, the main variation of the physical parameters across the discontinuity occurs on the direction $\boldsymbol{n}$ normal to the discontinuity itself. It can be shown that, if $S$ is the surface at the plasma boundary, any closed line integral across the surface yields (Baumjohann \& Treumann, 1996):

$$
\oint_{S} \frac{d X}{d n} d n=2[X]
$$

where $[X]=X_{2}-X_{1}$ is the variation of the quantity $X$ across the boundary. Defining $\langle X\rangle=\frac{1}{2}\left(X_{1}+X_{2}\right)$ the mean of $X$ across the discontinuity, we can write

$$
[A B]=\frac{1}{2}([A]\langle B\rangle+\langle A\rangle[B])
$$

In a system of reference moving with the discontinuity at speed $\boldsymbol{U}$, where the fluid propagates at speed $\boldsymbol{v}^{\prime}=\boldsymbol{v}-\boldsymbol{U}$, due to invariance under Galilean transformation the time derivative can be replaced as follows:

$$
\begin{aligned}
\frac{\partial}{\partial t} & =-\boldsymbol{U} \cdot \boldsymbol{\nabla} \\
& =-\boldsymbol{U} \cdot \boldsymbol{n} \frac{\partial}{\partial n} .
\end{aligned}
$$

In this frame of reference the jump conditions across the discontinuity can be written as:

$$
\begin{aligned}
& \boldsymbol{n} \cdot[n \boldsymbol{v}]=0 \\
& \boldsymbol{n} \cdot[n m \boldsymbol{v} \boldsymbol{v}]+\boldsymbol{n} \cdot\left[p+\frac{B^{2}}{2 \mu_{0}}\right]-\frac{1}{\mu_{0}} \boldsymbol{n} \cdot[\boldsymbol{B} \boldsymbol{B}]=0 \\
& {[\boldsymbol{n} \times \boldsymbol{v} \times \boldsymbol{B}]=0} \\
& \boldsymbol{n} \cdot[\boldsymbol{B}]=0 .
\end{aligned}
$$

Equation 3.31d implies that the component of the magnetic field normal to the discontinuity, $B_{n}$, is conserved, i.e.

$$
\left[B_{n}\right]=0 .
$$

The same holds for normal the mass flow. From Equation 3.31a it follows that

$$
\left[n v_{n}\right]=0 .
$$

Rewriting Equations 3.31b and 3.31c by separating the vectors in their components tangential and orthogonal to the discontinuity yields the so called Rankine-Hugoniot equations, which, given an equation of state, describe the boundary conditions at a discontinuity for ideal MHD plasmas. They are: 


$$
\begin{aligned}
& n m v_{n}\left[v_{n}\right]=-\left[p+\frac{B^{2}}{2 \mu_{0}}\right] \\
& n m v_{n}\left[\boldsymbol{v}_{\boldsymbol{t}}\right]=\frac{B_{n}}{\mu_{0}}\left[\boldsymbol{B}_{\boldsymbol{t}}\right] \\
& B_{n}\left[\boldsymbol{v}_{\boldsymbol{t}}\right]=\left[v_{n} \boldsymbol{B}_{\boldsymbol{t}}\right] .
\end{aligned}
$$

Different classes of discontinuities can be defined based of the properties of the solutions of Equations 3.34. They will be described in the following sections.

\subsubsection{Contact and tangential discontinuities}

They are the solutions corresponding to vanishing normal mass flux $n m v_{n}=0$, which implies $v_{n}=0$. For these solutions the Rankine-Hugoniot equations reduce to

$$
\begin{aligned}
& {\left[p+\frac{B^{2}}{2 \mu_{0}}\right]=0} \\
& n m v_{n}\left[\boldsymbol{v}_{\boldsymbol{t}}\right]=\frac{B_{n}}{\mu_{0}}\left[\boldsymbol{B}_{\boldsymbol{t}}\right] \\
& B_{n}\left[\boldsymbol{v}_{\boldsymbol{t}}\right]=\left[v_{n} \boldsymbol{B}_{\boldsymbol{t}}\right] .
\end{aligned}
$$

If the normal component of the magnetic field is continuous, i.e. $\left[B_{n}\right]=0$, the following conditions must be satisfied:

$$
\begin{aligned}
& {[p]=0} \\
& {\left[\boldsymbol{v}_{\boldsymbol{t}}\right]=0} \\
& {\left[B_{n}\right]=0} \\
& {\left[\boldsymbol{B}_{\boldsymbol{t}}\right]=0 .}
\end{aligned}
$$

This type of discontinuities are called contact discontinuities, and are characterized by constant pressure and magnetic field. The only quantity which varies across the discontinuity is the density. The plasmas on the two sides of the discontinuity are connected by the normal magnetic field, and propagate at the same tangential speed $\left[\boldsymbol{v}_{t}\right]$

If instead the normal component of the magnetic field is zero, $B_{n}=0$, then

$$
\left[p+\frac{B^{2}}{2 \mu_{0}}\right]=0 .
$$

Equation 3.37 implies pressure balance across the discontinuity. Discontinuities satisfying this condition are called tangential discontinuities. The magnetic and mass fluxes are constant, while the other quantities can vary freely. 


\subsubsection{Rotational discontinuities}

The second class of solutions are characterized by a finite mass flux

$$
n m v_{n}= \pm \frac{B_{n}}{\sqrt{\mu_{0}\left\langle(n m)^{-1}\right\rangle}}
$$

and a continuous velocity across the discontinuity $\left[v_{n}\right]=0$. They are called rotational discontinuities. For these solutions the density is constant across the boundary layer, and, since $n m v_{n} \neq 0$, from Equation 3.38 also follows that $B_{n} \neq 0$. The RankineHugoniot equations reduce to

$$
\begin{aligned}
& {\left[p+\frac{B^{2}}{2 \mu_{0}}\right]=0} \\
& n m v_{n}\left[\boldsymbol{v}_{\boldsymbol{t}}\right]=\frac{B_{n}}{\mu_{0}}\left[\boldsymbol{B}_{\boldsymbol{t}}\right] \\
& B_{n}\left[\boldsymbol{v}_{\boldsymbol{t}}\right]=\left[v_{n} \boldsymbol{B}_{\boldsymbol{t}}\right] .
\end{aligned}
$$

The first equation in 3.39 again implies that the total pressure is constant across the discontinuity. Using Equation 3.28 and the fact that $\left[v_{n}\right]=0$, the third relation can be rewritten as

$$
B_{n}\left[\boldsymbol{v}_{\boldsymbol{t}}\right]=v_{n}\left[\boldsymbol{B}_{\boldsymbol{t}}\right] .
$$

Due to the fact that both $v_{n}$ and $\boldsymbol{B}_{\boldsymbol{t}}$ are constant, the tangential components of the velocity and the magnetic field cannot change in magnitude. The two vectors rotate together in a plane tangential to the discontinuity. The set of conditions satisfied by rotational discontinuities is

$$
\begin{aligned}
& {[n]=0} \\
& {[p]=0} \\
& {\left[v_{n}\right]=0} \\
& {\left[B_{n}\right]=0} \\
& {\left[B^{2}\right]=0 .} \\
& {\left[\boldsymbol{v}_{\boldsymbol{t}}-\frac{\boldsymbol{B}_{t}}{\sqrt{\mu_{0} n m}}\right]=0 .}
\end{aligned}
$$

\subsubsection{Shocks}

The third and last class of solutions corresponds to shocks. They are such that 


$$
\left(n m v_{n}\right)^{2}=-\frac{1}{2}\left([p][n m]-\frac{\left\langle\boldsymbol{B}^{2}\right\rangle\langle n m\rangle}{\mu_{0}}\right) \pm \sqrt{\frac{\Delta}{4}},
$$

with

$$
\Delta=\left([p][n m]-\frac{\left\langle\boldsymbol{B}^{2}\right\rangle\langle n m\rangle}{\mu_{0}}\right)^{2}+\frac{4 B_{n}^{2}[p][n m]\langle n m\rangle}{\mu_{0}}
$$

The solution with $[\mathrm{nm}]=0$ corresponds to intermediate shocks. All other cases with a non-zero jump in density correspond to true shocks. Under the assumption that the density is discontinuous, Equation 3.42 has four solutions, of which only 3 are physical, as they correspond to a positive mass flux. One solution is such that $[p][n m]>0$, and two correspond to the case $[p][\mathrm{nm}]<0$. If the pressure increases across the discontinuity two solutions are associated to an increase in density, i.e. to compression, and one corresponds to $[\mathrm{nm}]<0$. The latter is called rarefaction shock.

Shocks can be subdivided in fast and slow shocks, which in turn can be parallel, oblique or perpendicular, depending on the orientation of the magnetic field relative to the normal to the discontinuity. Before describing the details of the different types of shocks, some general properties typical of this class of discontinuities will be discussed. Consider the the condition of vanishing mass flux $\left[n m v_{n}\right]=0$. Since the normal component of the velocity and the density are non-zero and have non-zero jumps, making use of 3.28 yields

$$
\left[v_{n}\right]=-\frac{\left\langle v_{n}\right\rangle}{\langle n\rangle}[n]
$$

indicating that normal velocity and density change across the discontinuity in an opposite way. Another interesting property of shocks can be derived considering Equation 3.34b and Equation 3.34c. Substituting for the jump in tangential velocity yields

$$
\left[v_{n} \boldsymbol{B}_{t}\right]=\frac{B_{n}^{2}}{\mu_{0} n m v_{n}}\left[B_{t}\right]
$$

The following cross product must then vanish

$$
\left[\boldsymbol{B}_{\boldsymbol{t}}\right] \times\left[v_{n} \boldsymbol{B}_{\boldsymbol{t}}\right]=0
$$

i.e.

$$
\left(v_{n 1}-v_{n 2}\right)\left(\boldsymbol{B}_{\boldsymbol{t 1}} \times \boldsymbol{B}_{\boldsymbol{t 2}}\right)=0 .
$$

The condition that the normal component of the velocity has a non-zero jump implies that the tangential magnetic fields on both sides of the shock are parallel to each other and coplanar with the shock normal. This property is actually employed for the determination of the direction of shock normal from in-situ magnetic field measurements. 


\section{Fast and slow shocks}

In general, shocks form in a medium when disturbances propagate at speeds higher than the propagation speed of waves in the medium itself. When disturbances propagate at speed faster than the slow magnetosonic speed slow shocks form. When disturbances propagate at speed faster than the fast magnetosonic speed, on the other hand, fast shocks form. Fast shocks are characterized by an increase in magnetic pressure, whereas across a slow shock the magnetic pressure decreases (hence the magnetic field strength). Moreover, for fast shocks the magnetic field is tilted towards the shock surface, while for slow shocks it rotates towards the shock normal. Depending on the angle between the direction of propagation of the shock and the magnetic field, shocks are referred to as parallel, perpendicular, or oblique shocks. For parallel shocks, since $\left[B_{n}\right]=0$, the magnetic field does not "feel" the effects of the shock. Its size and direction remain unchanged. Perpendicular shocks share the same property. In reality, though, small deviations are always present, leading to the development of turbulence.

This chapter described the derivation of the MHD equations and discussed the properties of ideal MHD plasmas and waves. MHD discontinuities were introduced and the different types of MHD shocks were presented. In the context of space physics MHD waves are observed, e.g., in EUV images of the Sun in the form of propagating brightness enhancements. Examples of MHD shocks are the terrestrial bow shock, which forms as a consequence of the interaction between the solar wind and the Earth's magnetosphere, and interplanetary CME-driven shocks. The latter are the object of the study presented in this work. Models that describe the shock location as a function of the shock parameters will be presented in Chapter 7 
4. The NASA STEREO mission 

This Chapter will provide an overview of the instruments on board the NASA Solar TErrestrial RElations Observatory (STEREO, Kaiser et al. 2008) mission, with particular focus on the imaging package Sun-Earth Connection Coronal and Heliospheric Investigation (SECCHI, Howard et al. 2008). Section 4.3 will describe the processing methods required in order to use SECCHI images for scientific analysis, as well as the techniques used to enhance contrast in difference images and to investigate the propagation of solar wind transients.

The NASA Solar TErrestrial RElations Observatory (STEREO, Kaiser et al. 2008) mission is a space based mission aimed at the understanding of the physical processes that govern the initiation and evolution of coronal mass ejections (Kaiser et al., 2008). It was launched on 25 October 2006 and it is comprised of two identical spacecraft, STEREO Ahead (A) and Behind (B), which orbit the Sun at 1 AU. STEREO A precedes the Earth in its orbit, and STEREO B follows it. The separation angle between each of the spacecraft and Earth increases by about $22^{\circ}$ per year (see Figure 4.1). The two different heliocentric orbits were achieved with the aid of multiple lunar gravity assists, which enabled the two spacecraft to be launched on a single vehicle. Each of the STEREO A and B spacecraft mounts an identical set of four instrument suites: the Sun-Earth Connection Coronal and Heliospheric Investigation (SECCHI, Howard et al. 2008), the Plasma and Suprathermal Ion Composition (PLASTIC, Galvin et al. 2008) instrument, the In-situ Measurements of Particles and CME Transients

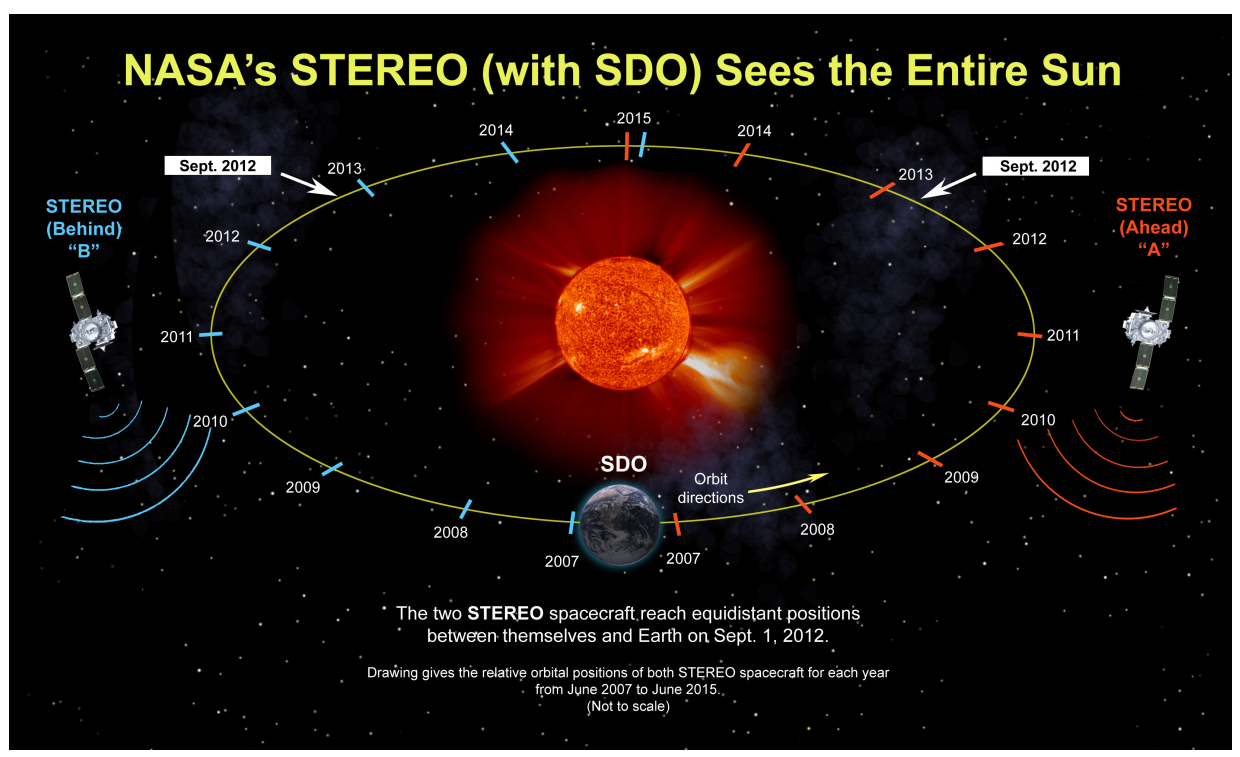

Figure 4.1.: Position of the STEREO spacecraft in different mission phases. Image courtesy of the NASA STEREO consortium. 


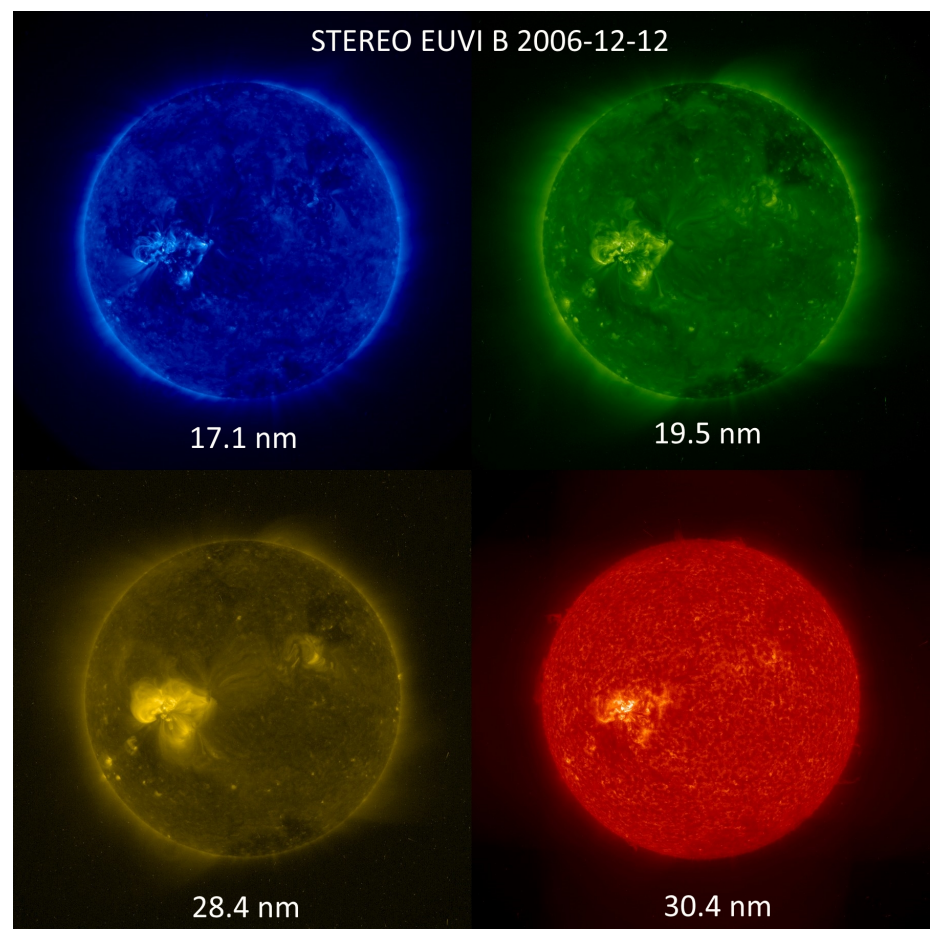

Figure 4.2.: First light STEREO-B EUVI images taken on 12 December 2006. Image courtesy of the NASA STEREO/SECCHI consortium.

(IMPACT, Luhmann et al. 2008) suite and the STEREO/WAVES (Bougeret et al., 2008) suite.

The multipoint nature of STEREO observations provides an unprecedented insight in the 3D structure of solar wind transients and on the spatial distribution of solar wind parameters. Combined data from the instruments on board STEREO can be used to address the main scientific goals of the mission. These include the understanding of the properties and mechanisms of CME eruption and interplanetary propagation, the study of the origin and evolution of solar energetic particles (SEP) events, and the development of a time-dependent, 3D model of the solar wind in terms of its magnetic field, temperature, density and velocity (see Kaiser et al. 2008). Additionally, STEREO beacon low cadence, low resolution data, can be used for space weather applications. Since the study presented in this work was based on the analysis of images from the instruments on board the SECCHI suite, the next section is dedicated to a detailed description of the instruments' characteristics.

\subsection{The SECCHI suite}

The SECCHI suite is a package composed of five imaging instruments, whose combined fields of view observe the entire space between Sun and Earth. The main scientific questions addressed by STEREO/SECCHI involve the understanding of the time scales of CME initiation and the identification of the structures which play a role in CME eruption, the determination of the $3 \mathrm{D}$ structure of coronal features like active regions, loops 
and helmet streamers, the study of the 3D morphology of CMEs and their kinematics, and the identification of the forces governing their propagation (Howard et al., 2008). The Extreme Ultraviolet Imager (EUVI) observes the upper chromosphere, transition region and corona in four wavelengths, corresponding to emission lines of ions formed at different temperatures (hence locations): the He II line at $30.4 \mathrm{~nm}$, the Fe XV line at $28.4 \mathrm{~nm}$, the Fe XII line at $19.5 \mathrm{~nm}$, and the Fe IX line at $17.1 \mathrm{~nm}$. The instrument's field of view is Sun-centred and extends out to $1.7 R_{\odot}$. Figure 4.2 shows STEREO-B EUVI first light images, taken on 12 December 2006, in the four wavelengths imaged by the instrument. A large active region is visible at low latitudes in all the wavelengths imaged by the instrument, with the overlying coronal loops. Coronal holes are visible at the poles in the form of regions of reduced EUV brightness. The different wavelengths reveal different structures on the Sun.

COR1 and COR2 are two occulted Lyot coronagraphs with nested fields of view imaging sunlight Thomson-scattered by electrons in the solar corona. COR1 is an internally occulted coronagraph, covering the range from $1.4 R_{\odot}$ to $4 R_{\odot}$. The internal occulter allows the achievement of better resolution near the limb, at the cost of higher stray light levels. The instrumental noise, though, being unpolarized, can be highly reduced by taking polarization images. Each COR1 observation is made up of three polarization brightness images. To avoid smearing due to the evolution of coronal structures the polarization brightness images are acquired within 11 seconds. The complete observations have a cadence of 8 minutes.

With an angular field of view of $8^{\circ}$, COR2 extends the COR1 field of view by imaging the corona between $2.5 R_{\odot}$ and $15 R_{\odot}$. The overlap in the COR2 and COR1 fields of view allows the continuous monitoring of propagating structures. The necessity for two separate coronagraphs is driven by the large spatial gradients in the coronal brightness at those heliocentric distances. The external occulter mounted on COR2 produces lower levels of stray light compared to those achieved by the internally-occulted COR1. This allows the instrument to obtain images of the corona at larger distances from the Sun, where the signal is weaker. Each COR2 image is obtained from three polarization images, which can be combined to compute total and polarization brightness ${ }^{1}$ images. The polarization sequence is obtained in 11 seconds, whereas the complete image cadence is 15 minutes, and the pixel size is 14.7 arcsec. Figure 4.3a shows composite EUVI 195, COR1 and COR2 observations of the 7 June 2011 CME.

The Heliospheric Imagers HI1 and HI2 extend the range of observations of the SECCHI suite all the way to Earth, taking snapshots of the inner heliosphere in white-light between $15 R_{\odot}$ and beyond $215 R_{\odot}$. Two separate instruments are necessary due to the high stray light gradients in the observational range of HI1 and HI2 (see Figure 4.4a). Due to the faint nature of interplanetary CMEs, the stray light and sensitivity requirements for the HI1 and HI2 are quite stringent. In particular stray light levels smaller than $\sim 3 \times 10^{-13} B_{\odot}$ are required for HI1, and $\sim 10^{-14} B_{\odot}$ for HI2, where $B_{\odot}$ is the average intensity of the solar disk. The instruments' sensitivity is driven by the CME brightness and is $\sim 3 \times 10^{-15} B_{\odot}$ for HI1 and $\sim \times 10^{-16} B_{\odot}$ for HI2. HI1 has a field of

\footnotetext{
${ }^{1}$ The polarization brightness is defined as the difference between the tangential and radial polarization components (Hayes et al., 2001)
} 


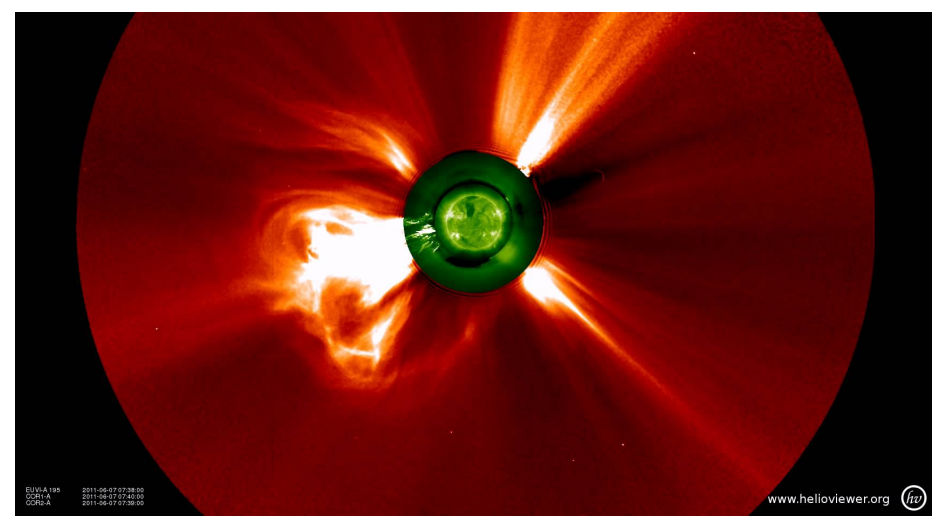

(a)

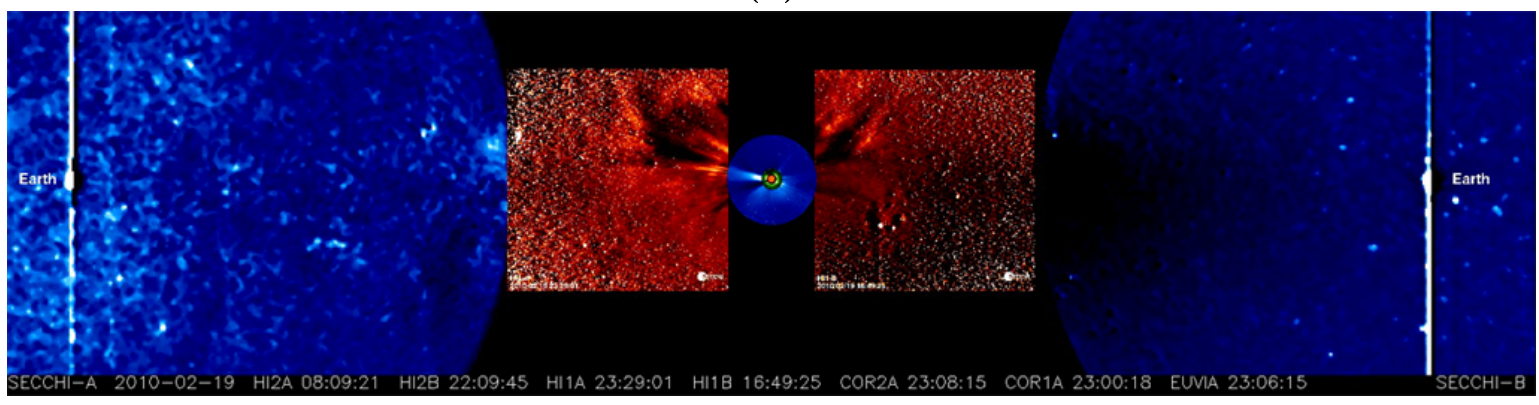

(b)

Figure 4.3.: Top panel: composite EUVI 195, COR1 and COR2 image of a CME erupted on 7 June 2011. Image credits: http://www.thesuntoday.org/tag/ solar-terrestrial-relations-observatory/. Bottom panel: STEREO A and B SECCHI complete field of view. The Earth is visible in the HI2 fields of view. Image credits: NASA STEREO/SECCHI consortium.

view of $20^{\circ}$, centred at $14^{\circ}$ away from the Sun. The image cadence is 30 minutes and the pixel size is 70 arcsec. HI2 has a field of view of $70^{\circ}$, with its centre at roughly $54^{\circ}$ away from the Sun, and takes a snapshot every 60 minutes. The long exposure times for the HI instruments are achieved via the sum of short CCD exposures, up to one minute. As a first step the electronic offset and cosmic ray impact signatures are removed from each short-exposure image. The 2048x2048 image thus obtained is binned down to a resolution of $1024 \times 1024$ and added to the others to derive the final raw image.

The opening angle from the solar equator for the two instruments is $45^{\circ}$, driven by the average angular size of a CME. Figure 4.3b illustrate the combined fields of view of all the instruments on board the SECCHI suite. Thanks to such observations the evolution of CMEs can be followed from their eruption on the solar surface all the way to Earth. The multipoint nature of the observations, whose potential can be increased even further when combining them with SOHO or SDO images, is a powerful tool for the investigation of the $3 \mathrm{D}$ properties of solar wind features. In situ measurements by WIND and ACE provide additional information on the physical properties of the plasma and magnetic field inside CMEs. Observing CMEs at the same time as they 
are detected in-situ allows the association between white-light features and the density and magnetic field structures detected in ACE and WIND data (see, for example Davis et al. 2009).

\subsection{The other instruments on board STEREO}

Besides the SECCHI suite, each of the spacecraft of the NASA stereo mission mounts 3 additional instrument packages. The Plasma and Suprathermal Ion Composition Composition (PLASTIC, Galvin et al. 2008) instrument is dedicated to the study of solar wind protons and ion composition, as well as the spectra, composition and anisotropy of suprathermal electrons with energies in the range between 0.3 to $80 \mathrm{keV} / \mathrm{e}$. Suprathermal electrons are indicators of the magnetic field topology in the solar wind. Bidirectional streams, for example, are often found in association with CMEs, and are thought to be indicative of electrons travelling back and forth along closed magnetic field lines. The In-situ Measurements of Particles and CME Transients (IMPACT, Luhmann et al. 2008) suite consists of seven instruments arranged in two suites: the boom suite and the SEP packages. Included in the boom suite are the Solar Wind Electron Analyzer (SWEA), the Suprathermal Electron Telescope (STE) and the Magnetometer (MAG). The SEP package is comprised of a Solar Electron Proton Telescope (SEPT), a Suprathermal Ion Telescope (SIT), a Low Energy Telescope (LET), and a High Energy Telescope (HET). The instruments on board the IMPACT suite measure the in-situ properties of magnetic fields, energetic particles and electrons and will help gaining deeper insight in the details of the magnetic field topology, solar energetic particle acceleration by flares and shocks, also in combination with radio measurements. STEREO/WAVES (Bougeret et al., 2008) is a radio burst tracker able to detect Type II and III radio bursts, both remotely and in-situ. These are respectively associated to CMEs and flares. Measurements from the radio antennas mounted on STEREO/WAVES enable scientists, for example, to track CME-driven shocks and determine their spatial extent, and to study the genesis of radio bursts.

\subsection{Analysis of STEREO/SECCHI Observations}

Image processing is fundamental in the analysis of STEREO/SECCHI observations. It must be applied to both coronagraph and heliospheric imagers data. Raw images are processed through the IDL SECCHI PREP routine, part of the IDL SolarSoft library. The library includes routines, databases and utilities for data analysis in the context of solar and heliospheric physics (for more info visit http://www.lmsal.com/ solarsoft/). The SECCHI_PREP routine comes with a number of options which allow different types of processing, the specific corrections depending on the imaging instrument. For COR 2 the following corrections are performed (see also sohowww. nascom.nasa.gov/solarsoft/stereo/secchi/doc/cor_prep.html):

- flat field and photometry corrections: flat field correction is applied to compen- 
sate for the non uniform response of the CCD. This can be caused by a number of factors, like changes in the optics efficiency, pixel to pixel response variations, vignetting or the presence of debris. These effects are compensated for by the SECCHI_PREP routine through calibration images. Correction for optical distortion is also performed;

- conversion of pixel values to units of Mean Solar Brightness via multiplication by a calibrating factor;

- CCD bias subtraction;

- division by exposure time;

- correction for on board image processing by the SECCHI electronic box (SEB): corrections are applied for any on board processing other than image compression.

A number of optional procedures are also available. For example, it is possible to rotate COR2 images to solar north (the default alignment is to ecliptic north).

For HI1 and HI2, on the other hand, the follow corrections are applied (Eyles et al., 2009):

- cosmic ray scrubbing values removal: the heliosperic imagers require long exposure times (20 minutes for HI1 and 1 hour for HI2) to sufficiently reduce the signal to noise ratio. In order to avoid building up of hits by cosmic rays, these long exposures are obtained summing up shorter ones. corrections have to be performed to remove cosmic ray events which accumulate due to the long exposure times required for HI1 and HI2 (respectively 20 minutes and 1 hour). These are partially applied on board and partially taken care of through the SECCHI_PREP routine;

- Blooming removal: blooming and saturation around bright objects occurs when their signal exceeds the pixel capacity; In such cases vertical bleeding of charge takes place between adjacent pixels, causing the appearance of vertical stripes in the images. The values associated to saturated pixels, as well as to missing data blocks, are appropriately replaced by the SECCHI_PREP routine;

- Correction for shutterless readout: smearing in the images occurs due to the absence of shutters on the HI cameras and the consequent CCD exposure during readout. The SECCHI_PREP corrects these effects;

- Flat field and photometry corrections: see above;

- Update of optic and pointing parameters: since August 2008 the pointing parameters are obtained using background stars in HI images. The corresponding parameters in the images headers are now updated according to the attitude solutions currently implemented. 


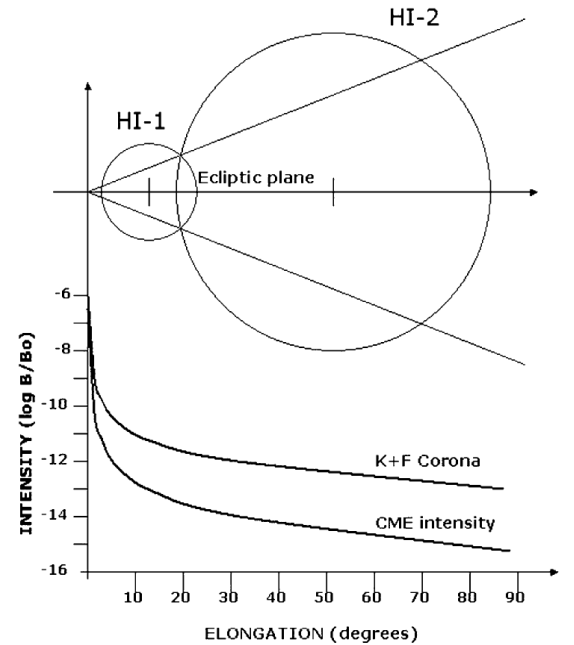

(a)
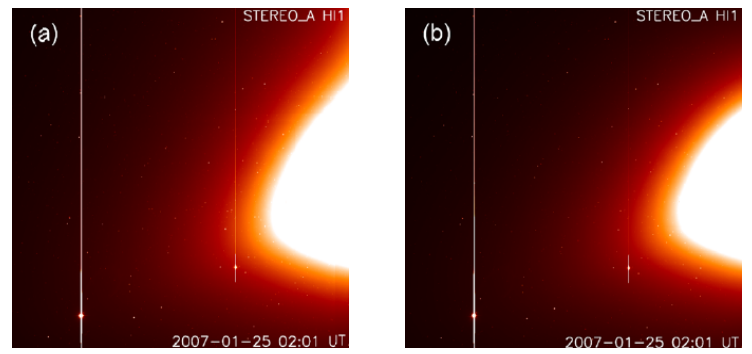

(c)

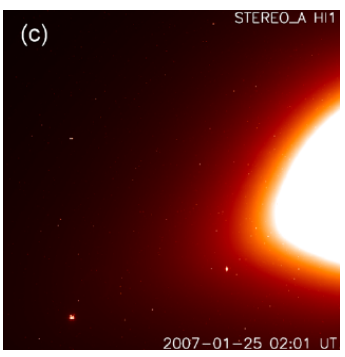

(d)

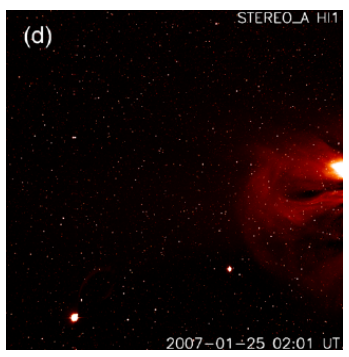

(e)

Figure 4.4.: Top panel: Typical brightness of a CME compared to the cumulative brightness of the $\mathrm{K}$ and $\mathrm{F}$ corona as a function of angular distance from the Sun. The fields of view of the heliospheric imagers HI1 and HI2 are included. The intensity curves clearly show that the background coronal signal dominates over the signal produced by evolving CMEs. Bottom panel: subsequent steps necessary to extract the CME signal from HI1 and HI2 images. Reprinted from Eyles et al. (2009) with the permission of AIP publishing. Figure 4.4b is a raw HI1 image taken on 25 January 2007, Figure 4.4c and is obtained by correcting for flatfield and shutterless readout and Figure $4.4 \mathrm{~d}$ is derived by removing blooming around planets. Figure 4.4e shows the signal of the underlying CME, extracted via background subtraction. Reprinted from Eyles et al. (2009) with the permission of AIP publishing.

For HI1 and HI2 images, additionally, background subtraction is necessary to extract the signal of CMEs due to their low brightness compared to the signal of the background corona (Eyles et al., 2009). Figure 4.4a shows a comparison between the typical intensity of CMEs and the one of the $\mathrm{K}$ and $\mathrm{F}$ corona, normalized to the intensity of the solar disk. The intensities are shown as a function of the angular distance from the Sun, and the fields of view of HI1 and HI2 are also included. The plot clearly shows that in order to extract the CME signal it is necessary to eliminate the dominating coronal contribution. This is done by building background images that will be subtracted to CME observations in order to remove the signal from the $\mathrm{F}$ and $\mathrm{K}$ corona. 


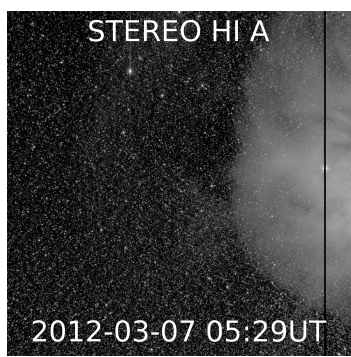

(a)

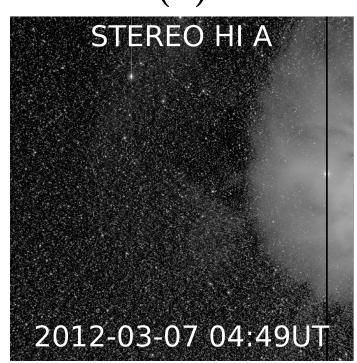

(b)

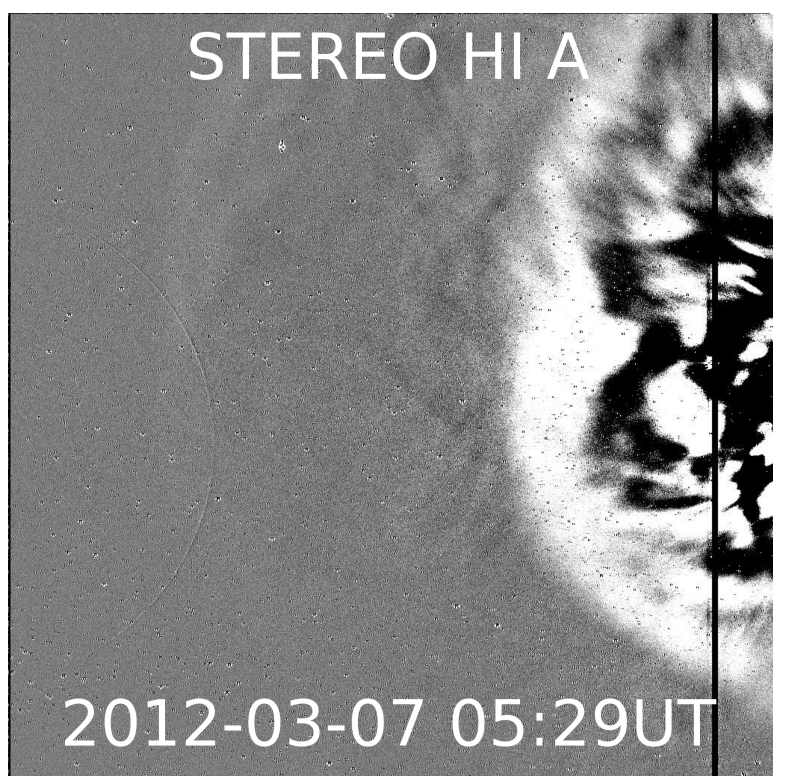

(c)

Figure 4.5.: Left panel: running difference image (4.5c) built by subtracting two subsequent HI1 A snapshots of a propagating CME (4.5a and 4.5b).

Star removal is performed by aligning the images in the Geocentric Equatorial Inertial (GEI) system previous to subtraction (Davies et al., 2009). Background images are built by taking the average brightness in each pixel on a time scale of 1 day for HI1 observations and 11 days for HI2 observations. The underlying assumption is that the time scales of variations in the $\mathrm{F}$ and $\mathrm{K}$ corona intensity are much larger than the CME crossing time in the imagers' fields of view. Figure 4.4 illustrates the steps performed to extract the signal of a CME. The raw HI1 A image (Figure 4.4b) is corrected for flat field and shutterless readout (Figure 4.4c). Figure $4.4 \mathrm{~d}$ results from the removal of blooming around the planets, and the CME is finally visible after background removal in Figure 4.4e. Background-subtracted HI images are available for download on the UK Solar System Data Centre (UKSSDC) website ${ }^{2}$.

After image processing has been performed, remote-sensing observations of solar wind flows like CIRs and CMEs can be used to determine their kinematics. A widely employed technique to increase the contrast in white-light images and to analyse the proper motion of solar wind flows is the construction of difference images (Davies et al., 2009, Sheeley et al., 1999, 1997), obtained subtracting an image with the previous one in time. In the resulting image the white features precede the dark ones in the direction of motion. Since what is seen in difference images is the brightness variation resulting from the motion of the CME, white areas correspond to areas of density increase, whereas dark areas correspond to regions of depletion. Difference images are an effective alternative way to remove the signal of the background corona. Furthermore, they can be used to enhance the visibility of faint features, (like, e.g, the intensity increase due to compression downstream of CME-driven shocks) in the field of view of a coro-

\footnotetext{
$2_{\text {www.ukssdc.rl.ac.uk/solar/stereo/data.html }}$
} 


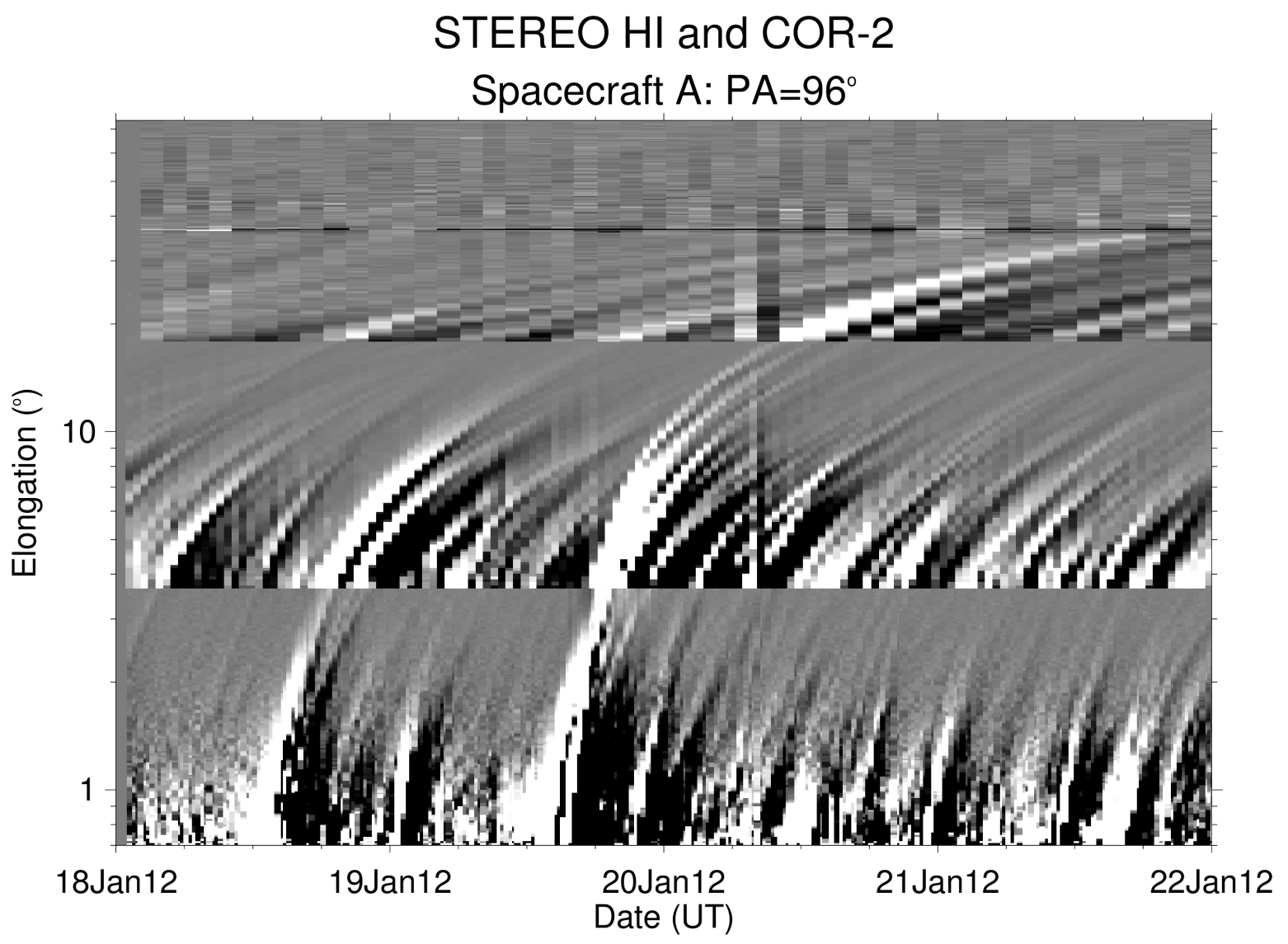

Figure 4.6.: Time elongation plot obtained from STEREO A COR2, HI1 and HI2 observations between 18 and 22 January 2012.

nagraph or heliospheric imager. An example is illustrated in Figure 4.5. Figure 4.5c is obtained subtracting the signal in $4.5 \mathrm{~b}$ from $4.5 \mathrm{a}$ : The comparison between the images clearly shows how the contrast is enhanced by the implementation of this technique. It is important to notice that the features seen in difference images do not delineate the CME structure, but show the variation of density due to CME motion.

Difference images can be used to build time-elongation plots, also called j-maps (Davies et al., 2009, Sheeley et al., 1999). These are obtained by juxtaposition of slices taken from time sequences of difference images at a fixed position angle. The technique was introduced by Sheeley et al. (1999), and since then it has been widely employed for the analysis of the evolution of solar wind features. The result is a map of intensity as a function of elongation and time, in which outward propagating features are visible as curved tracks. An example is shown in Figure 4.6, built from slices of STEREO A COR2, HI1, and HI2 observations cut along the Ecliptic plane. The tracks appearing on 18 and 19 January correspond to CMEs travelling away from the Sun.

It should be noted (Sheeley et al., 1999, 2008) that even in the case of propagation at constant speed, due to geometric effects, the elongation angle measured by an observer at a fixed distance from the Sun is not a linear function of time. As a result curved tracks arise in time-elongation plots even in absence of acceleration in the CME motion. 


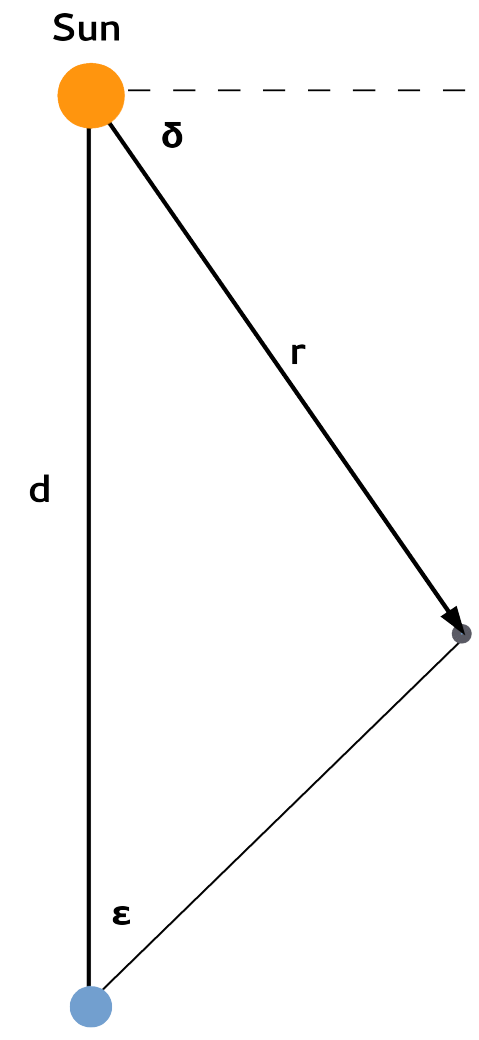

Observer

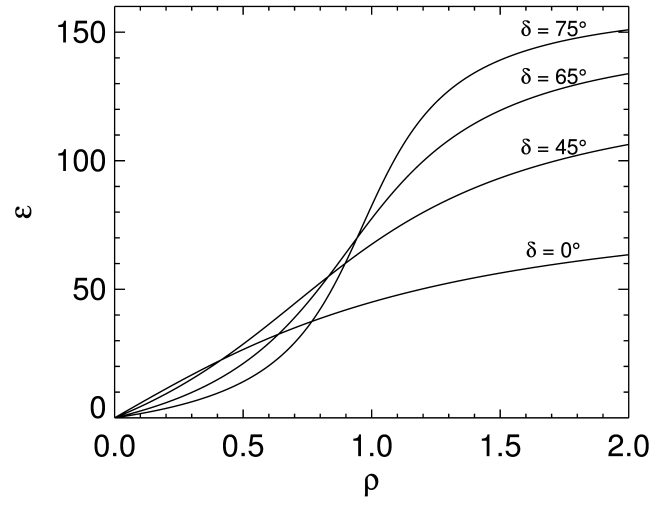

(b)

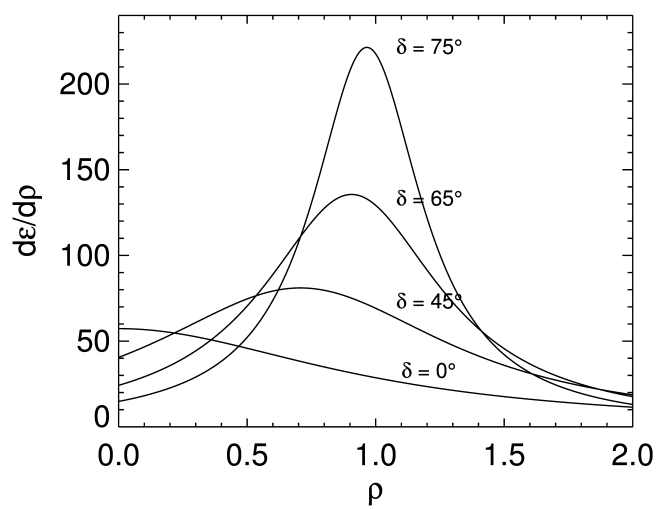

(c)

(a)

Figure 4.7.: Left panel: sketch of a CME seen as a point source moving at an angle $\delta$ from the plane of the Sky, seen by an observer at a distance $d$ from the Sun. Adapted from Sheeley et al. (1999) Right panel: elongation angle as a function of distance for different CME propagation angles (Top). Bottom: $\frac{d \epsilon}{d \rho}$ as a function of distance for different CME propagation angles.

In fact, consider a CME moving at constant speed at an angle $\delta$ from the plane of the sky (see Figure 4.7a). Let $\epsilon$ be the elongation measured by an observer at a distance $d$ from the Sun, and $r$ the CME-Sun distance. Applying the law of sines yields

$$
\frac{r}{d}=\frac{\sin \epsilon}{\cos (\epsilon-\delta)}
$$

with $r$ being the CME height and $d$ the distance between Sun and observer. Defining $\rho=\frac{r}{d}$ yields

$$
\tan \epsilon=\frac{\rho \cos \delta}{1-\rho \sin \delta}
$$

If the CME propagates at constant speed, then 


$$
\rho=\frac{v_{r}\left(t-t_{0}\right)}{d},
$$

where $t_{0}$ is the eruption time (i.e. the time at which $r=0$ ) and the $v_{r}$ de-projected radial speed.

The behaviour of the elongation as a function of distance for the Sun for different propagation angles is shown in Figure 4.7b, along with the slope of the curves $\epsilon(\rho)$ (Figure 4.7c) given by

$$
\frac{d \epsilon}{d \rho}=\frac{\cos \delta}{1-2 \rho \sin \delta+\rho^{2}},
$$

see Sheeley et al. (1999). The plots show that, for propagation at angles close to the plane of the sky, the elongation profile starts with a slope corresponding to the true radial speed and flattens out. For propagation closer to the direction of the observer the apparent angular motion starts with a slope corresponding to speeds lower that the true radial speed. The curves steepen rather quickly until they reach apparent speeds higher than $v_{r}$, before flattening again. An intuitive explanation of such behaviour is given by Sheeley et al. (1999): "If a projectile is shot well out of the sky plane in the general direction of the observer (or in the opposite direction), there will be a contribution to the apparent acceleration even if the projectile moves at constant speed. It is easy to appreciate this if one realizes that the only observable quantity is the change of apparent position with respect to time. Thus an object shot nearly at the observer will not show much angular change while it is far away but will require a rapid neck-wrenching motion to follow when it sails past the observer".

The present chapter introduced the NASA STEREO mission and its instruments. Details on the image processing and data analysis techniques were provided. The methods used to determine the kinematics of propagating solar wind features via the analysis of STEREO/SECCHI images are illustrated in Chapter 6 

5. On the Three Dimensional Structure of Coronal Mass Ejections 

This chapter introduces models for the 3D CME geometry and the observational basis for their development. Particular focus is given to the Graduated Cylindrical Shell model (GCS) developed by Thernisien et al. (2006). Additional models such as the circular cone model and the elliptical cone model are reviewed at the end of the chapter.

\subsection{CMEs as cylindrical flux ropes}

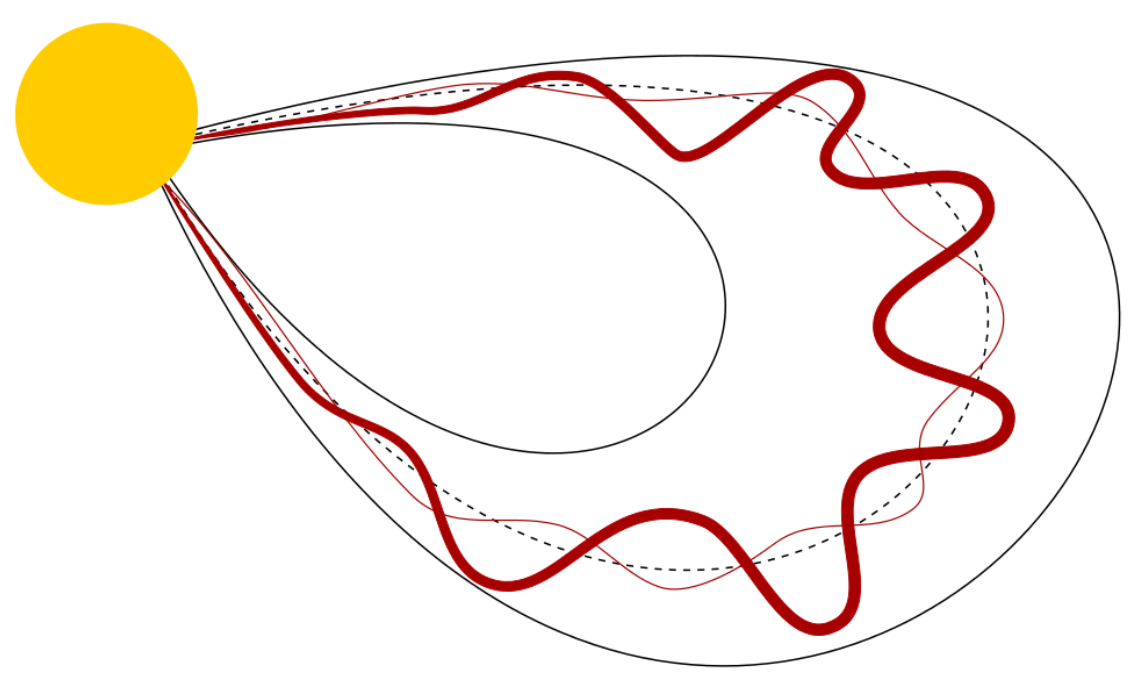

Figure 5.1.: Graphical representation of a CME seen as an expanding magnetic flux rope.

Since the first CME observations in the early 70s, the details of their 3D structure have been a matter of debate. The issue arises from the fact that coronagraphs and heliospheric imagers detect Thomson-scattered radiation, and the resulting brightness is integrated along the line of sight. What is observed is therefore a projection of the "true" CME structure on a 2D surface. The typical CME morphology is the three part structure, with a bright leading edge followed by a dark cavity and a bright core, but for a long time it was unclear whether CMEs are bubbles, flux rope systems, or even 2D objects (see Cremades \& Bothmer 2004 and references therein). Associations between CMEs observed at the Sun and magnetic clouds signatures in in-situ data 


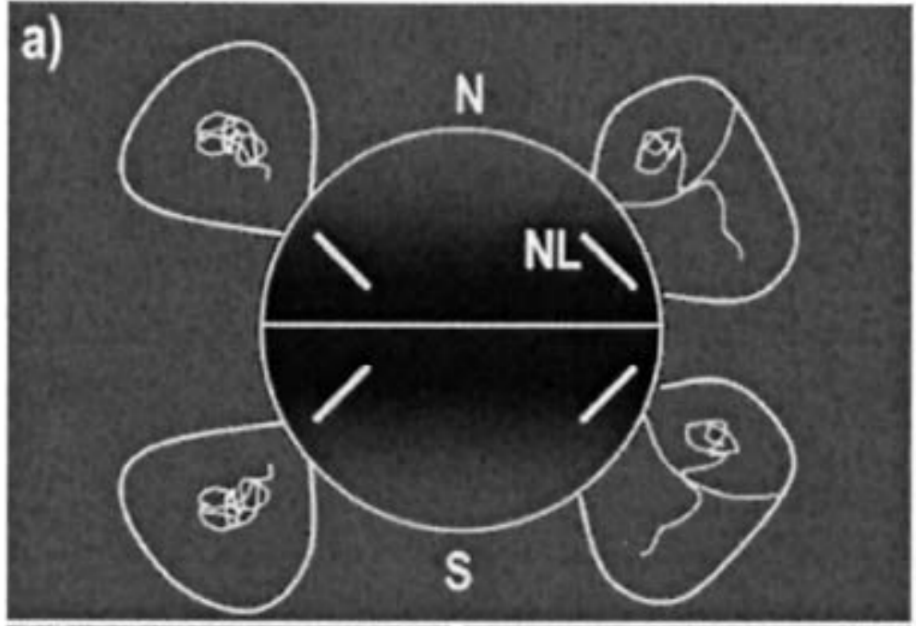

(a)
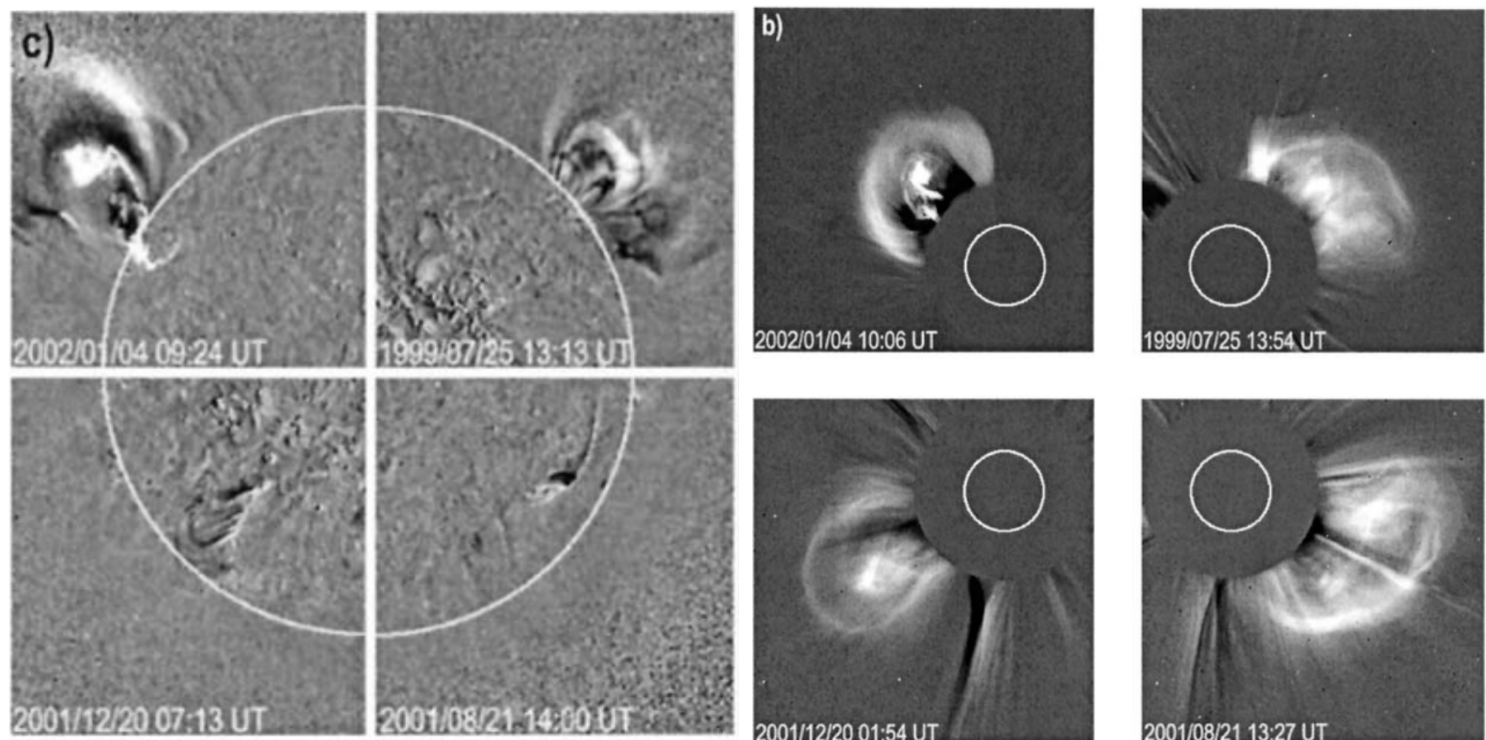

(b)
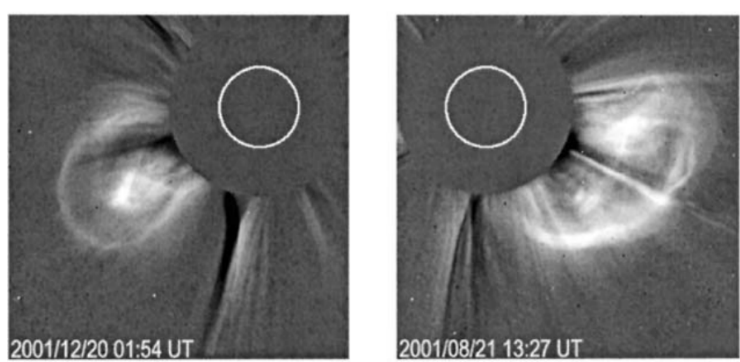

(c)

Figure 5.2.: Top panel: graphical representation of the appearance of front-sided CMEs in relation to the orientation of their source region's neutral line (indicated as NL in the picture). Bottom panel: SOHO/LASCO C2 observations of four CMEs (right) and their solar source regions (left) matching the scheme in Figure 5.2a. From Cremades \& Bothmer (2004). C)ESO, reproduced with permission.

(Bothmer \& Schwenn, 1994) suggest that CMEs are twisted flux ropes connected to the Sun (see, e.g., Chen et al. 2000). In order to shed light on the 3D properties of CMEs, Cremades \& Bothmer (2004) analysed LASCO MDI, EIT and C2 observations of 124 CMEs which exhibit clear white-light structure. The size and orientation of the photospheric source regions were determined, as well as the position angle and the angular width of the CMEs. The analysis of CMEs and the corresponding source regions revealed that the observed morphology was related to the orientation of the source region neutral line, i.e. the line that separates opposite magnetic polarities. 
They found that the typical orientation of source region neutral line is NE to SW in the northern hemisphere and NW to SE in the southern hemisphere. Moreover, they observed that CMEs with SR neutral line parallel to the limb exhibit entirely different observational features compared to those whose SR neutral line was perpendicular to the limb. They interpreted this as evidence for a cylindrically symmetric expanding flux rope system. Under this hypothesis, if the orientation of the neutral lines follows Joy's law ${ }^{1}$, front sided events on the East limb in the northern hemisphere would have their neutral lines perpendicular to the limb, and the flux rope would be observed along its axis of symmetry. On the other hand, events on the West limb would have their neutral line parallel to the limb, and would be observed edge-on, i.e. with their axis orthogonal to the line of sight. The opposite configuration would be expected in the southern hemisphere due to the typical SR neutral line orientation, in accordance to Hale's law ${ }^{2}$. A sketch of the configuration described above is provided in Figure 5.2a. Figures 5.2c shows the remarkable agreement between model and observations. The association was found to be stronger for events arising from longer-lived source regions with larger dimensions. A relation was found between length of the source region and the angular width of the CME, with scatter due to projection effects.

The work of Cremades \& Bothmer (2004) showed that CMEs are expanding cylindrical structures whose characteristics in white-light observations can be directly related to the properties of their photospheric source regions, namely their position on the solar disk and the orientation of the neutral line. This work paved the way for the development of the Graduated Cylindrical Shell model, which was designed to reproduce the geometry of CMEs in white-light coronagraph images. The model will be discussed in the next section.

\subsection{The graduated cylindrical shell model}

Following the work by Cremades \& Bothmer (2004), and the evidence supporting the hypothesis that CMEs expand self-similarly (see, e.g. Chen et al. 1997, 2000), Thernisien et al. (2006) developed a geometrical model to describe the CME morphology according to the flux rope paradigm. The Graduated Cylindrical Shell (GCS) model represents the CME as a tube of varying circular cross section sitting on two conical legs (see Figure 5.3). The whole system is assumed to expand self-similarly. The model can be also used to derive the electron density. The geometry of the GCS is fully described by the following three parameters: the height of the conic legs $h$, the aspect ratio $k$, which encodes the condition of self similar expansion, and the half angular width $\alpha$, i.e. the angle describing the opening of the conical legs. All the other geometrical parameters are derived as a function of the quantities just introduced. The height of

\footnotetext{
1 "The following spot of the pair tends to appear farther from the equator than the preceding spot, and the higher the latitude, the greater is the inclination of the axis to the equator", (Hale et al., 1919).

2 "the preceding and following spots of binary groups, with few exceptions, are of opposite polarity, and that the corresponding spots of such groups in the Northern and Southern hemispheres are also of opposite sign. Furthermore, the spots of the present cycle are opposite in polarity to those of the last cycle", (Hale et al., 1919).
} 


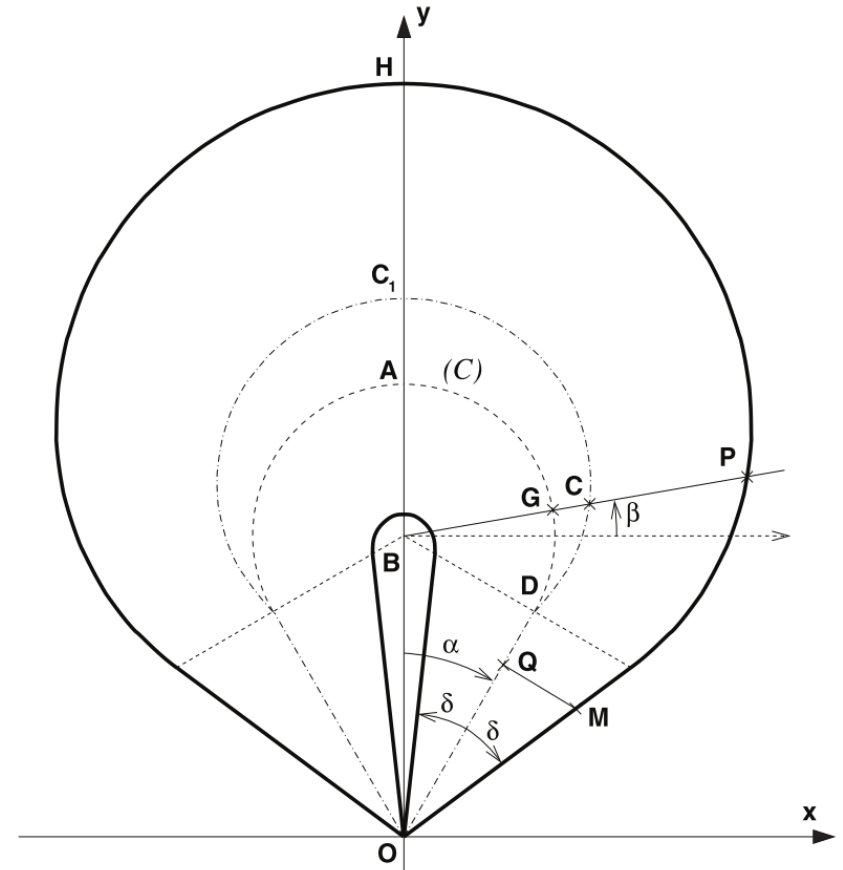

(a)

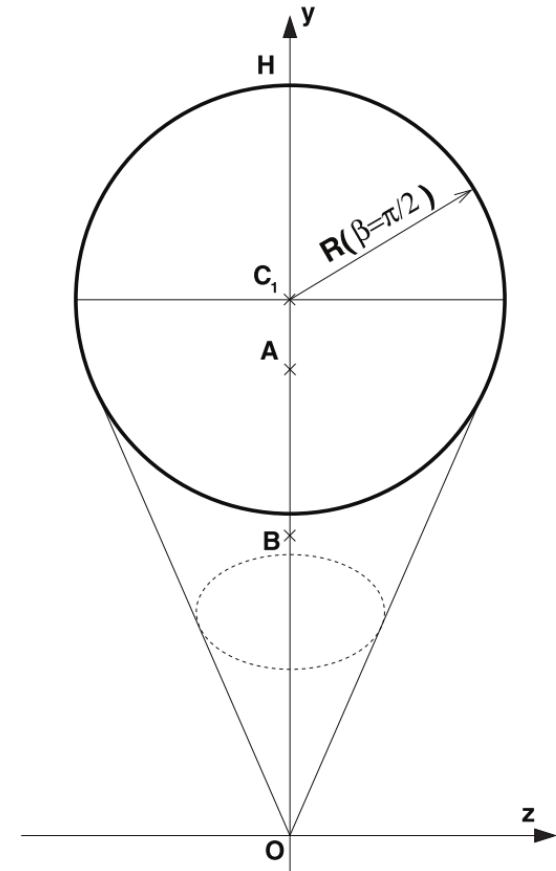

(b)

Figure 5.3.: Face-on (left) and edge-on (right) view of a flux rope modelled via the Graduated Cylindrical Shell model. From Thernisien (2011). C AAS. Reproduced with permission.

the apex, for example, is given by

$$
h_{\text {front }}=h \frac{1+k}{1-k^{2}} \frac{1+\sin \alpha}{\cos \alpha} .
$$

The position of the flux rope in 3D space is described by the tilt angle of the source region's neutral line $\gamma$, the Carrington longitude $\phi$ and the heliographic latitude $\theta$ of the source region (see Figure 5.4). The tilt angle increases counter-clockwise, and $\gamma=0^{\circ}$ corresponds to a vertical neutral line. A full analytical description of the model geometry is provided in Thernisien (2011). The model can be used to fit the geometry of CMEs in coronagraph images by adjusting the parameters $\alpha, k$ and the CME height, as well as the orientation of the flux rope, to match the observations. Once the CME geometry is determined, synthetic coronagraph images can be produced if an electron density model is provided. Thernisien et al. (2006) used an asymmetric Gaussian profile, determined as:

$$
N_{e}(d)=N_{e} e^{\left(-\frac{d-a}{\sigma_{s}}\right)^{2}},
$$

where

$$
\sigma_{s}= \begin{cases}\sigma_{\text {trailing }} & \text { if } d<a \\ \sigma_{\text {leading }} & \text { if } d \geq a\end{cases}
$$




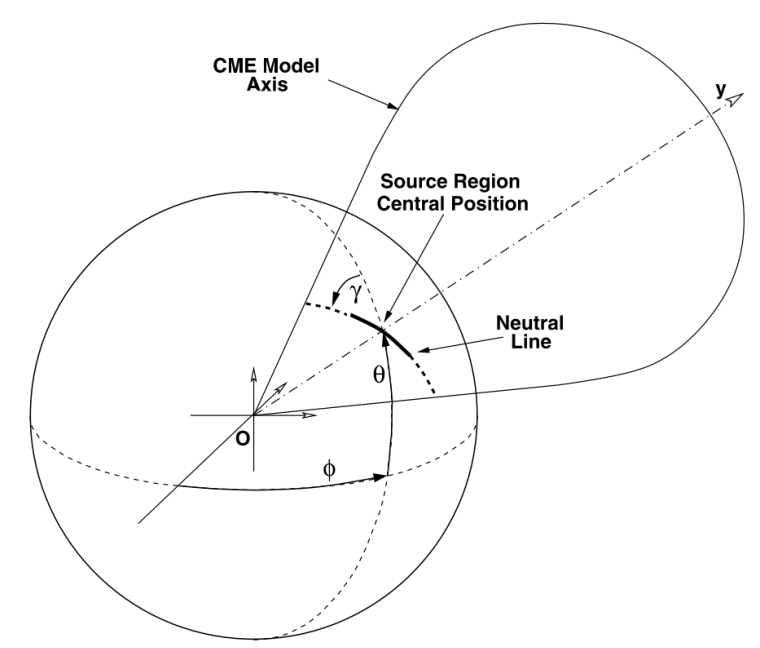

Figure 5.4.: Orientation of the GCS flux rope in 3D. From Thernisien et al. (2006). CCAAS. Reproduced with permission.

and $a$ is the radius of the circular cross section of the model leading edge (the segment $\overline{G P}$ in Figure 5.3a). The relation for the brightness due to Thompson scattering, namely

$$
B=C \int_{L O S} N_{e}(\mathbf{r}) G(r, \Omega) d z
$$

can then be inverted in order to determine the values of $N_{e}, \sigma_{\text {trailing }}$ and $\sigma_{\text {leading }}$ that yield the best fit to the observed brightness. The integral in Equation 5.3 is the integral along the line of sight of the electron density times a geometrical function $G(r, \Omega)$. The quantity $r$ is the size of the position vector $\mathbf{r}$ of a point in the corona. $\Omega$ is the angle between the line of sight vector and the vector originating at the centre of the Sun and culminating on a point along the line of sight. Figure 5.5 shows examples of synthetic coronagraph images obtained via GCS fitting of LASCO observations, and a comparison of the observed and fitted electron density profiles computed along a slit in the images (Thernisien et al., 2006). The model, apart from the initial slope, reproduces well the observed electron density.

Modelling CMEs via the GCS model has been employed in several applications. Bosman et al. (2012) selected 51 well structured CMEs imaged by STEREO A and B and modelled them as GCS flux ropes. They compared the CME orientation to those of their source regions and found that in $82 \%$ of the cases the CMEs undergo deflection towards the solar equator. This had already been pointed out by Cremades \& Bothmer (2004). The work presented by Bosman et al. (2012) was then expanded and included in a database of 241 CMEs fitted with the GCS in the field of view of STEREO COR2 $\mathrm{A}$ and B (http://www.affects-fp7.eu/cme-database/database.php). The work was carried on under the SOlar TERrestrial Investigation and Archives (SOTERIA), Advanced Forecast For Ensuring Communications Through Space (AFFECTS), and the HELiospheric Cataloging Analysis and Techniques Service (HELCATS) programs. Within the HELCATS program, moreover, the GCS model was used to determine the 

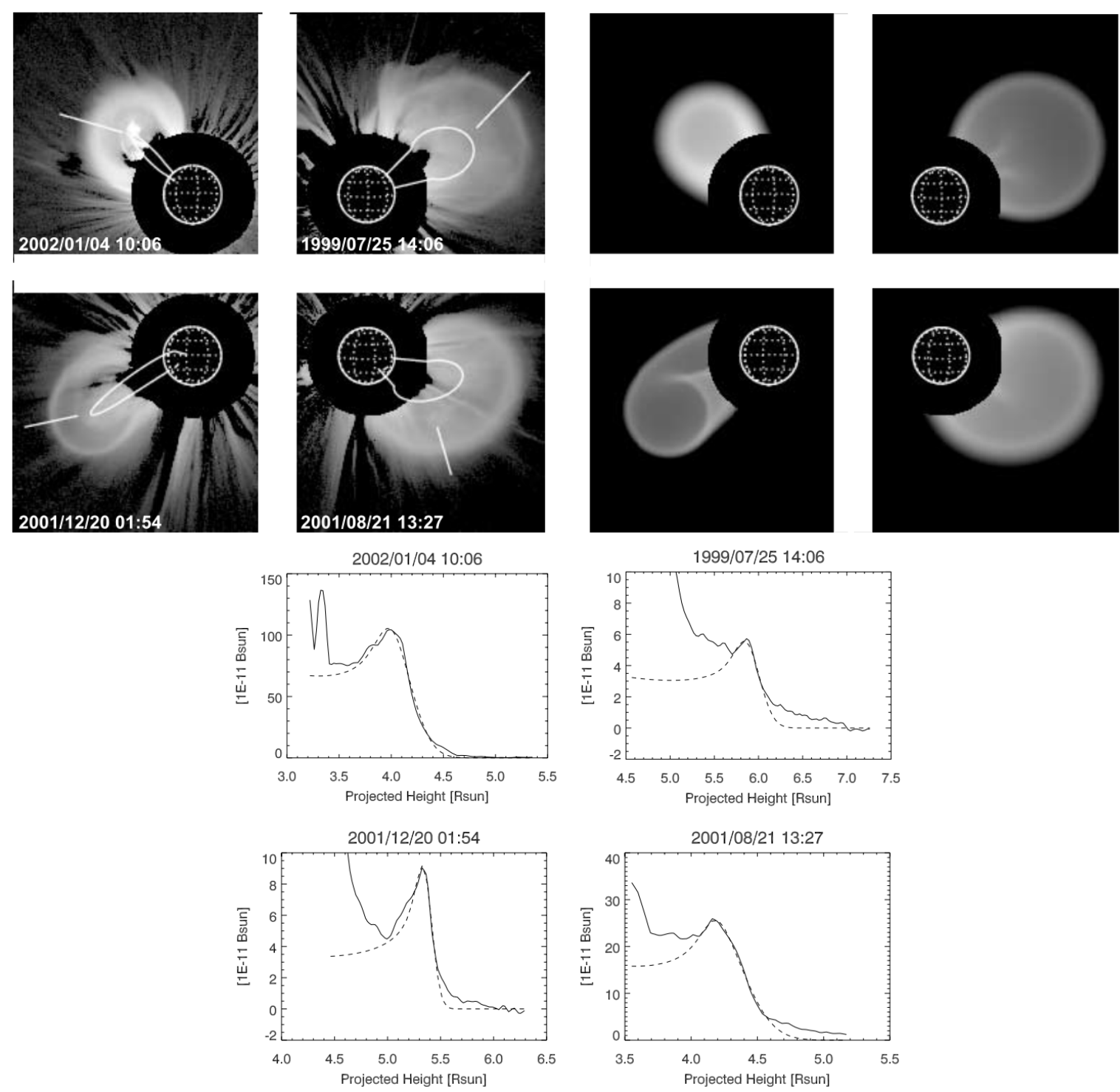

Figure 5.5.: Top panel: white-light (left) and synthetic (right) coronagraph images of four CMEs. The synthetic images are obtained via a GCS fit to the observed CME brightness profiles. Bottom panel: comparison between the observed electron density (solid line) and the one obtained by assigning a Gaussian profile to the GCS croissant (dashed line). From Thernisien et al. (2006). (C)AAS. Reproduced with permission.

speed of the CME apex for over 100 CMEs. Colaninno et al. (2013) used GCS fits to STEREO A and B and LASCO C2 observations to derive speeds and arrival times of interplanetary CMEs. Poomvises et al. (2012) applied the GCS to STEREO A and $\mathrm{B}$ and SOHO LASCO C2 images of CMEs and CME-driven shocks to infer the shock parameters using the models presented in Chapter 7. In this work we extend the COR2 GCS flux ropes in the AFFECTS database to the HI1 field of view for the events presented in Chapter 8, to investigate whether they indeed evolve self similarly. 


\subsection{Other geometrical models}

For the sake of completeness additional models for the CME geometry will be described. They were developed in order to investigate the 3D structure of CMEs and to derive their de-projected heights and speed from single point observations.

\subsubsection{Circular cone model}

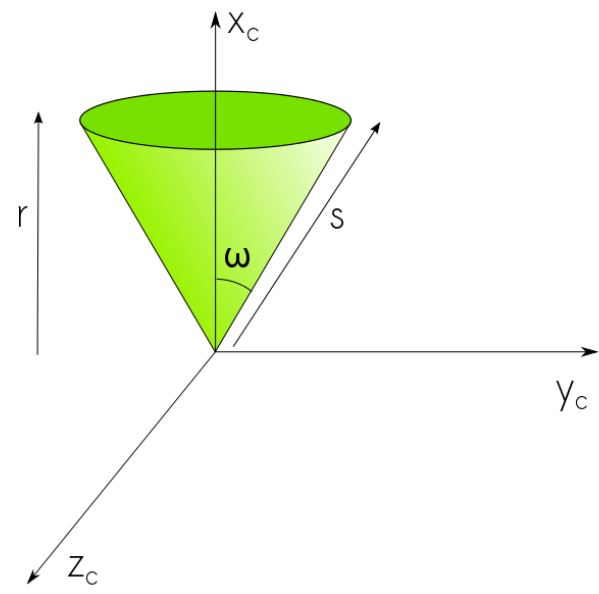

(a)
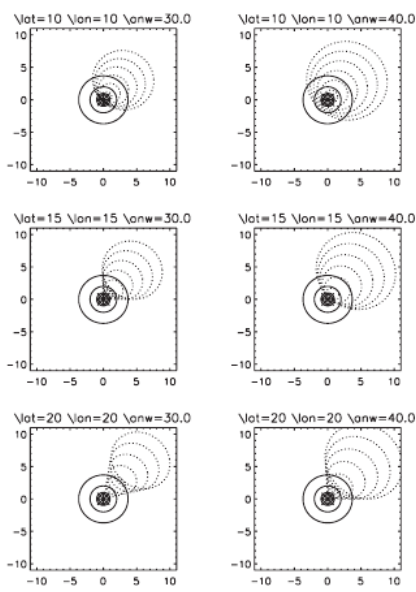

(b)
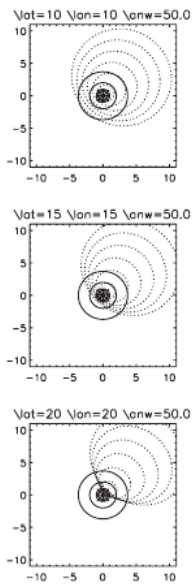

Figure 5.6.: Ice cream cone model for CMEs (Figure 5.6a, adapted from Zhao et al. (2002)), and its projection on the plane of the sky (Figure 5.6b, from Zhao et al. (2002)).

Developed by Zhao et al. (2002), the circular cone model was intended to fit halo CMEs. It was based on the observation that broadsided CMEs (i.e. with latitudinal span less than $120^{\circ}$ ) show a certain degree of circular symmetry and that they seem to maintain a constant angular width as they expand outwards. The simplest geometry that retains these properties is that of a cone of circular cross section. A cone of height $r$ and half-width $\omega$ (see Figure 5.6a) is described in Cartesian coordinates as

$$
\begin{aligned}
& x_{c}=s \cos \omega \\
& y_{c}=s \sin \omega \cos \delta \\
& z_{c}=s \sin \omega \sin \delta,
\end{aligned}
$$

with $\delta$ varying in the $z_{c} y_{c}$ plane between $0^{\circ}$ and $360^{\circ}$. The system $x_{c} y_{c} z_{c}$ has its origin at the Sun, the $x_{c}$ axis aligned to the axis of the cone, and the $x_{c} y_{c}$ plane aligned with the corresponding plane in heliographic coordinates. The cone is completely determined if values for $s$ and $\omega$ are provided. The projection of the cone on the plane of sky (see Figure 5.6b) can be computed, and it depends on the orientation of the cone's axis in the heliographic system of coordinates (Zhao et al., 2002). The latter is described in terms of the angles $\lambda$ and $\phi^{3}$. A cone can be fitted to the observed CME profiles in

\footnotetext{
${ }^{3}$ These angles are different from the $\lambda$ and $\phi$ introduced in Chapter 6
} 
LASCO observations by varying the three angles $\omega, \lambda$ and $\phi$. The system is degenerate in the case in which the cone axis is aligned to the line of sight. For a complete discussion of the model the reader can refer to Zhao et al. (2002)

\subsubsection{Elliptical cone model}
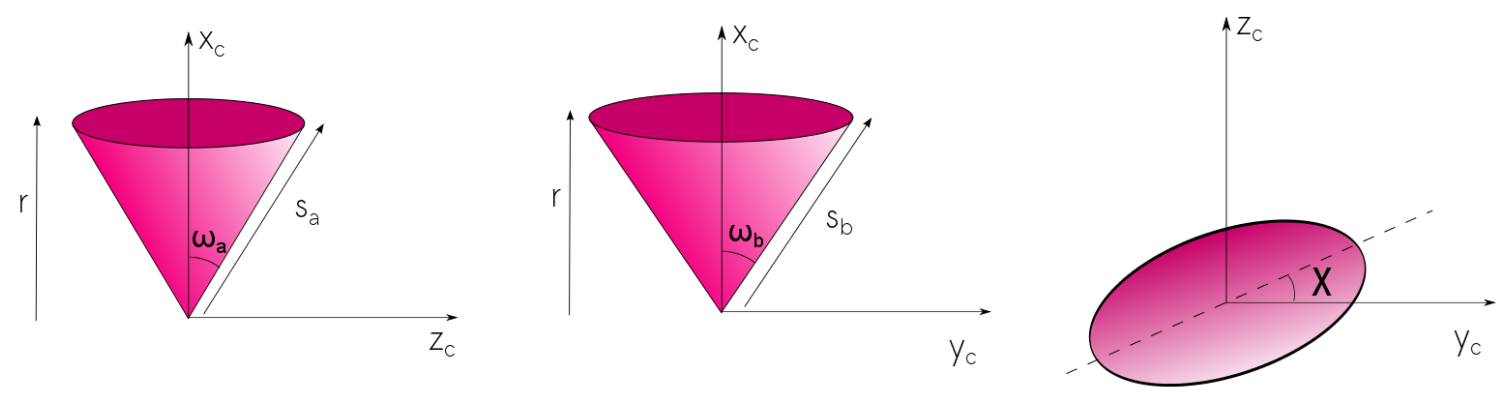

Figure 5.7.: Elliptical cone model for CMEs. Adapted from Cremades \& Bothmer (2005a)

The attempt to fit the CMEs analysed in Cremades \& Bothmer (2004) with the circular cone model suggested that an elliptical geometry would be more appropriate to represent the observed profiles of halo CMEs (Cremades \& Bothmer, 2005a,b). It should be noted that the projection of the circular cone on the plane of the sky is an ellipse whose eccentricity increases with increasing inclination of the cone w.r.t. the line of sight (see Figure 5.6b). However, based on their fitting results, Cremades \& Bothmer (2005a,b) hypothesized that a model with intrinsic eccentricity would be able to better reproduce the observed asymmetric halo CMEs. The elliptical cone model is characterized by two angular widths, $\omega_{a}$ and $\omega_{b}$, defined respectively in the direction of the semi-minor and semi-major axis (see Figure 5.7). In a coordinate system analogous to the one defined for the circular cone model the equations of the elliptical cone are given by

$$
\begin{aligned}
& x_{c}=s_{a} \cos \omega_{a} \\
& y_{c}=s_{b} \sin \omega_{b} \cos \delta \cos \chi+s_{a} \sin \omega_{a} \sin \delta \sin \chi \\
& z_{c}=s_{a} \sin \omega_{a} \sin \delta \cos \chi-s_{b} \sin \omega_{b} \cos \delta \sin \chi
\end{aligned}
$$

$\chi$ is the angle between the semi-major axis and the $y_{c}$ axis. Again, based on the orientation of the cone axis in the heliographic coordinate system it is possible to compute the projection of the ellipse in the plane of the sky. The system is closed, however the solution is not unique. A fit between the model and a set of 32 halo CMEs indeed revealed an asymmetry in the observed events, with an average ratio between 
the half-widths $<\frac{\omega_{b}}{\omega_{a}}=1.1>$ (Cremades \& Bothmer, 2005a,b).

This chapter presented several geometrical models developed in order to describe the 3D CME geometry. Such models can be used to reconstruct the $3 D$ geometry of CMEs from white-light images, which only show a 2D projection of the "true" 3D density distribution. Particular focus was given to the GCS model, which represents CMEs as self-similarly expanding flux ropes. The CME front is described as a curved cylinder with varying cross section, and the CME legs are conical. In this work the GCS model was applied to STEREO/SECCHI COR2 and HI1 observations in order to verify the assumption of self-similar propagation up to distances of $\simeq 87 R_{\odot}$. The application of the GCS model to STEREO/SECCHI observations is presented in Chapter 8. 

6. Models for the CME kinematics 

The kinematics of outward propagating features in a coronagraph or heliospheric imager field of view can be determined by converting the measured elongation angles to radial distances, if a geometric description of the tracked solar wind feature is provided. The elongation angles can then be expressed as a function of the feature's height and direction of propagation with respect to the Sun-Earth line. For single spacecraft observations a fit is required, under the assumption of propagation at constant speed and constant direction. For multipoint observations, on the other hand, the system of equations is closed, and the aforementioned assumptions can be relaxed. This chapter will provide an overview of the available methods, with particular focus given to the one employed in this work.

\subsection{Single Spacecraft Observations}

Before the advent of the STEREO mission, observations of CMEs were possible only from a single vantage point. Due to the optical thinness of the corona the details of the $3 \mathrm{D}$ structure and evolution of CMEs remained uncertain. This section will present an overview of the methods used to derive the kinematics of CMEs from single viewpoint observations. As mentioned in Chapter 4.3 such models were introduced by Sheeley et al. (1999), who proposed time-elongation plots as a way to follow the propagation of solar wind transients at a fixed position angle.

\subsubsection{The Fixed- $\phi$ Fitting Method}

Let's consider a single plasma element propagating at a fixed direction $\phi$, and let $\epsilon$ be the elongation angle measured by an observer at a distance $d$ from the Sun. The heliocentric distance of the plasma element can be derived as:

$$
r=d \frac{\sin \epsilon}{\sin (\epsilon+\phi)}
$$

(Sheeley et al., 1999, 2008). Under the assumption that the plasma element moves at constant velocity $v=\frac{r}{t}$ the measured elongation angle can be expressed as

$$
\epsilon=\arctan \left(\frac{v t \sin \phi}{d-v t \cos \phi}\right)
$$

The measured elongation-time profile can then be fitted to Equation 6.2 to infer $v$ and $\phi$. The fit also yields the eruption time $t_{0}$, i.e. the time at which $\epsilon=0$. The arrival time $t_{\text {arr }}$ at Earth, or at any give location, can easily be inferred. Due to the assumption of constant velocity and direction of propagation, the values derived with 


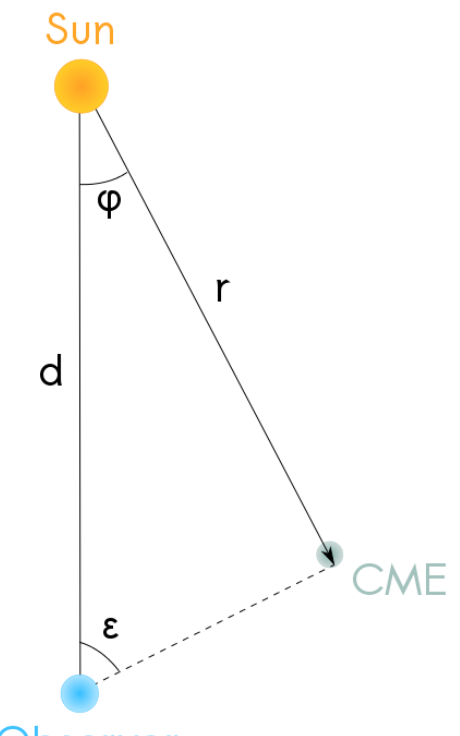

Figure 6.1.: Graphical representation of the fixed- $\phi$ approximation. Adapted from Sheeley et al. (1999)

this method (and all other single spacecraft fitting methods) should be thought of as averages over the CME propagation. It is important to remark that considering the CME as a point source implies assuming a very narrow longitudinal width, or that the same exact feature is tracked at all times.

\subsubsection{The Harmonic Mean Fitting Method}

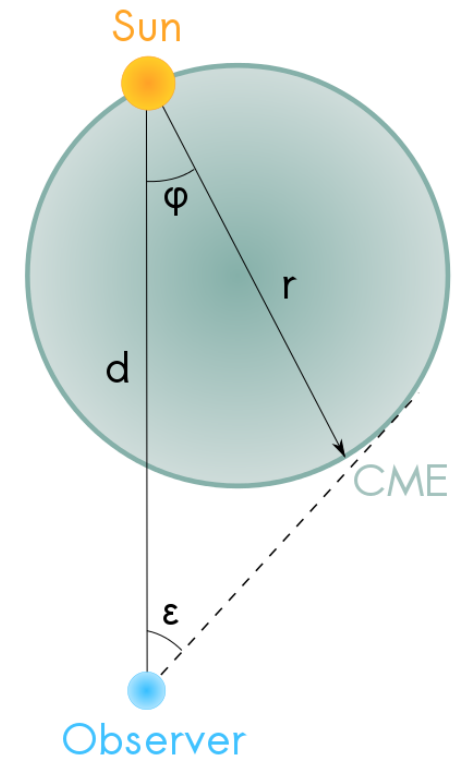

Figure 6.2.: Graphical representation of the harmonic mean approximation. Adapted from Lugaz et al. (2009). 
Developed by Lugaz et al. (2009) and Howard \& Tappin (2009), the harmonic mean method represents the CME as a sphere anchored at the Sun whose centre propagates radially in the direction $\phi$. The elongation angle $\epsilon$ is the angle between the Sun-observer line and the tangent to the circular front. The height of the harmonic mean circle is given by

$$
r=2 d \frac{\sin \epsilon}{1+\sin (\epsilon+\phi)},
$$

with $d$ being the distance between the Sun and the imaging spacecraft. Under the assumptions of constant propagation velocity, Equation 6.3 can be inverted to get the temporal evolution of the elongation angle:

$$
\epsilon=\arctan \left(\frac{v t \sin \phi}{2 d-v t \cos \phi}\right)+\arcsin \left(\frac{v t}{\sqrt{(2 d-v t \cos \phi)^{2}+(v t \sin \phi)^{2}}}\right) .
$$

Again, the (average) velocity and direction of propagation, eruption and arrival times in-situ can be obtained by fitting Equation 6.4 to the observed time-elongation profile.

\subsubsection{The Self-Similar Expansion Fitting Method}

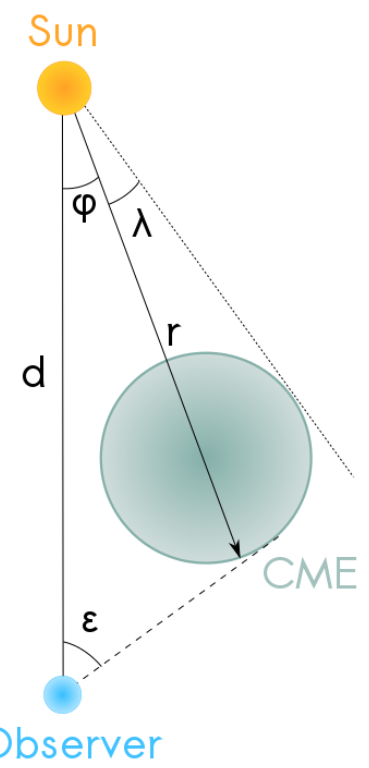

Figure 6.3.: Graphical representation of the self-similar expansion model. Adapted from Davies et al. (2012)

The fixed- $\phi$ and harmonic mean methods use two opposite and extreme geometries to derive the kinematics of propagating solar wind features: the first assumes a point-like source, and the second represents the CMEs as possessing very large angular widths. Davies et al. (2012) developed a generalized model describing the CME front as a circle expanding self-similarly (for further discussion on the model the reader can refer to 
Möstl \& Davies 2013). The technique is referred to as Self-Similar Expansion Fitting (SSEF). The SSEF circle, in contrast to the harmonic mean approximation, is not anchored at the Sun. The CME size increases as it travels outward, in agreement with observations, but the angular width $2 \lambda$ remain constant (hence the self-similarity). The distance $r$ of a CME of half-width $\lambda$ propagating in a direction $\phi$, seen under an angle $\epsilon$ from an observer at a distance $d$ from the Sun is:

$$
r=\frac{d \sin \epsilon(1+\sin \lambda)}{\sin \lambda+\sin (\epsilon+\phi)} .
$$

Inverting Equation 6.5 under the assumption of constant propagation speed yields

$$
\epsilon=\arccos \left(\frac{-b c+a \sqrt{a^{2}+b^{2}-c^{2}}}{a^{2}+b^{2}}\right),
$$

with

$$
a=\frac{d(1+c)}{v t}-\cos \phi, \quad b=\sin \phi, \quad c=\sin (\lambda) .
$$

The fixed- $\phi$ and harmonic mean approximation are limiting cases of the self-similarexpansion model, respectively for $\lambda=0^{\circ}$ and $\lambda=90^{\circ}$ Fitting the model to the observed elongations yields not $v, \phi, t_{0}$ and $t_{\text {arr }}$, but also the half-width of the $\operatorname{CME} \lambda$.

\subsection{Multi Spacecraft Observations}

All the techniques presented in this section are extensions of the corresponding single spacecraft methods. Having a second set of observations removes the necessity to fit the measured time-elongation pairs to the relations expressing the elongation angles in terms of time elapsed since eruption, speed and direction of propagation (Equations 6.2, 6.4, and 6.6). With the extra information coming from the additional viewpoint the equations for the unknown direction of propagation and speed can be directly solved without the need to assume radial propagation and constant velocity. This could have a strong impact on the prediction of arrival times, as stated by Lugaz \& Kintner (2013). The fitting techniques, in fact, cannot account for the effects of solar wind drag, which accelerates slow CMEs and slows down fast CMEs towards the solar wind speed (see, e.g. Gopalswamy et al. 2000). An analysis of the performance of the single spacecraft fixed- $\phi$ and harmonic mean methods in the presence of solar wind drag (Lugaz et al., 2010) revealed that neglecting acceleration leads to errors in the determined direction and speed of propagation, with the harmonic mean method exhibiting the largest discrepancies. The errors in the estimated arrival times can be as high as 12 hours for both methods.

Möstl et al. (2014) performed a study on the predictive capabilities of the three singlespacecraft fitting methods presented in Section 6.1. They derived in-situ arrival times and speeds from HI1 A observations of 22 coronal mass ejections, and compared the results to solar wind data provided by the WIND and STEREO-B spacecraft. They found no significant difference between the three methods, with arrival times being 
typically earlier than the observed ones by $8 \pm 6$ hours, and arrival speeds exceeding measured ones by $248 \pm 288 \mathrm{~km} / \mathrm{s}$. The result is somehow expected, since the models neglect the effects of deceleration. Colaninno et al. (2013) reported on the study of arrival time determinations obtained using multipoint observations of nine Earthimpacting CMEs. Their results support the hypothesis of a gradual deceleration of the analysed CMEs during their interplanetary propagation.

The extension of the single spacecraft methods introduced in Section 6.1 to multipoint observations of propagating solar wind features is discussed in the next sections.

\subsubsection{The Geometric Triangulation Method}

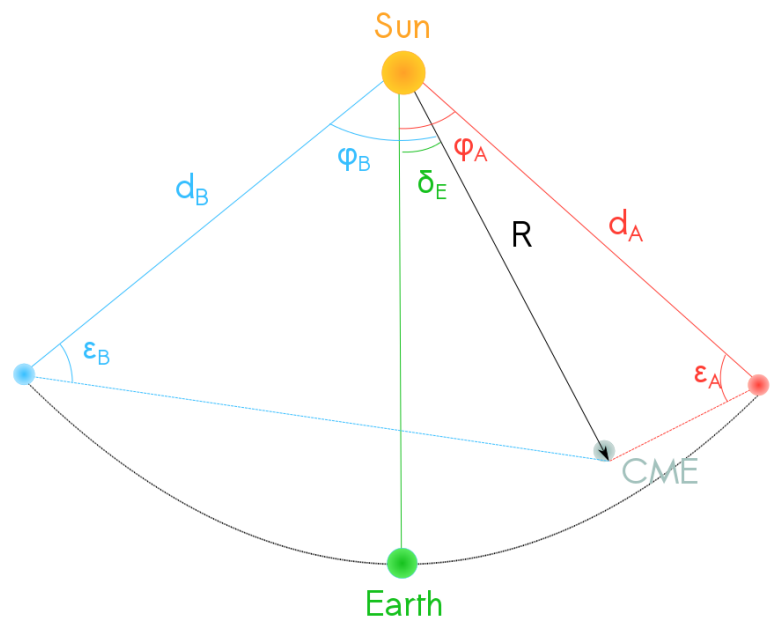

Figure 6.4.: Graphical representation of the geometrical triangulation method. Adapted from Liu et al. (2010a).

Developed by Liu et al. (2010a), the geometric triangulation method yields the temporal evolution of the height, direction of propagation and speed of solar wind features from the analysis of pairs of simultaneous time-elongation plots. It is the extension of the fixed- $\phi$ method for single-spacecraft observations. Its geometry is illustrated in Figure 6.1. $R$ is the heliocentric distance of the CME, $d_{A}$ and $d_{B}$ are the (known) distances between Sun and STEREO A and B respectively, $\phi_{A}$ and $\phi_{B}$ are the directions of propagation of the CME with respect to STEREO $\mathrm{A}$ or $\mathrm{B}$, and $\epsilon_{A}$ and $\epsilon_{B}$ are the elongation angles measured from STEREO A and B. It is possible to show that these quantities are related according to:

$$
\begin{aligned}
& d_{A}=R \frac{\sin \left(\sin \epsilon_{A}+\phi_{A}\right)}{\sin \epsilon_{A}} \\
& d_{B}=R \frac{\sin \left(\sin \epsilon_{B}+\phi_{B}\right)}{\sin \epsilon_{B}} . \\
& \gamma_{A B}=\phi_{A}+\phi_{B} .
\end{aligned}
$$

The angle $\gamma_{A B}$ is the known angular separation between STEREO A and B. The system of equations 6.8 can be directly solved for the three unknown quantities $R$, 
$\phi_{A}$ and $\phi_{B}$. The model considers a point-like geometry, the underlying assumption being that the two spacecraft observe at all times the same feature. This hypothesis is hindered by projection and Thompson scattering effects, which are minimized for CMEs propagating symmetrically with respect to the two imaging instruments.

\subsubsection{The Tangent to a Sphere Method}

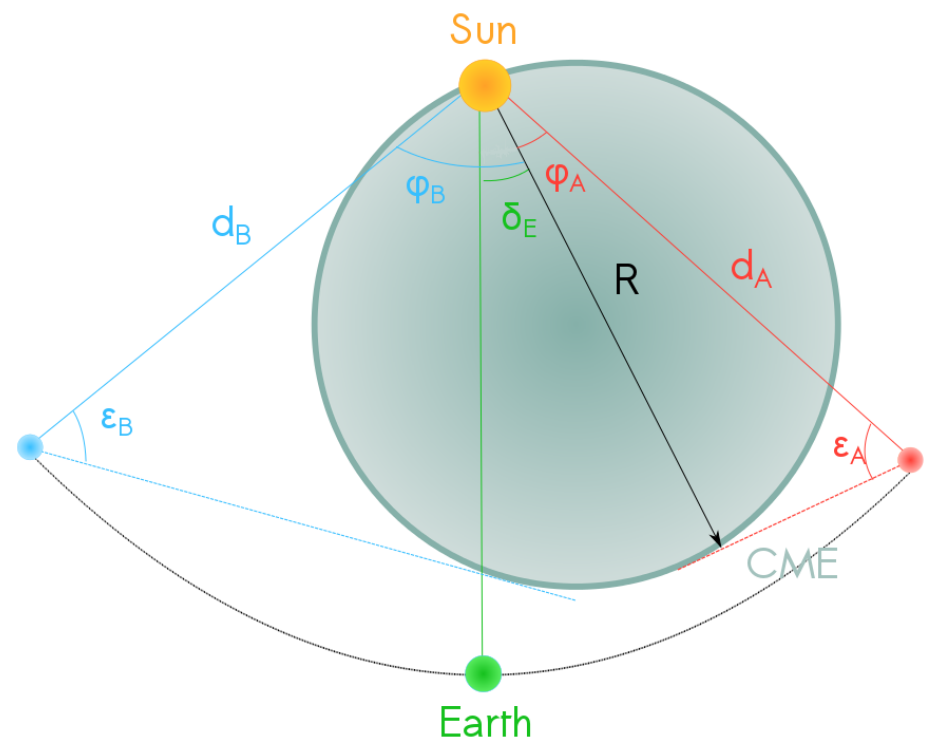

Figure 6.5.: Graphical representation of the tangent to a sphere approximation. Adapted from Lugaz et al. (2010).

The method represents the CME as a circular front anchored at the Sun, and was developed by Lugaz et al. (2010). Its geometry is presented in figure 6.5. With the same naming convention as above, it can be shown that the radius of the circle approximating the CME (which coincides with the CME height above the Sun) can be obtained as

$$
\begin{aligned}
& R=2 d_{A} \frac{\sin \epsilon_{A}}{1+\sin \left(\sin \epsilon_{A}+\phi_{A}-\delta_{E}\right)} \\
& R=2 d_{B} \frac{\sin \epsilon_{B}}{1+\sin \left(\sin \epsilon_{B}+\phi_{B}-\delta_{E}\right)} .
\end{aligned}
$$

$\delta_{E}$ is the CME direction of propagation with respect to the Earth-Sun line. It can be obtained from the position of the imaging spacecraft, known at all times, and the direction of propagation of the CME with respect to it. This condition closes the system of equations 6.9. The tangent to a sphere method, contrarily to the Liu et al. (2010a), does not require that STEREO A and B observe the same feature.

\subsubsection{The Stereoscopic Self-Similar Expansion model}

Similarly to the single viewpoint based methods, the tangent to a sphere and the triangulation technique employ two very extreme CME geometries. Davies et al. (2013) 
extended the self-similar expansion fitting introduced in Section 6.1.3 to the Stereoscopic Self-Similar Expansion Model (SSSEM, see Figure 6.6).

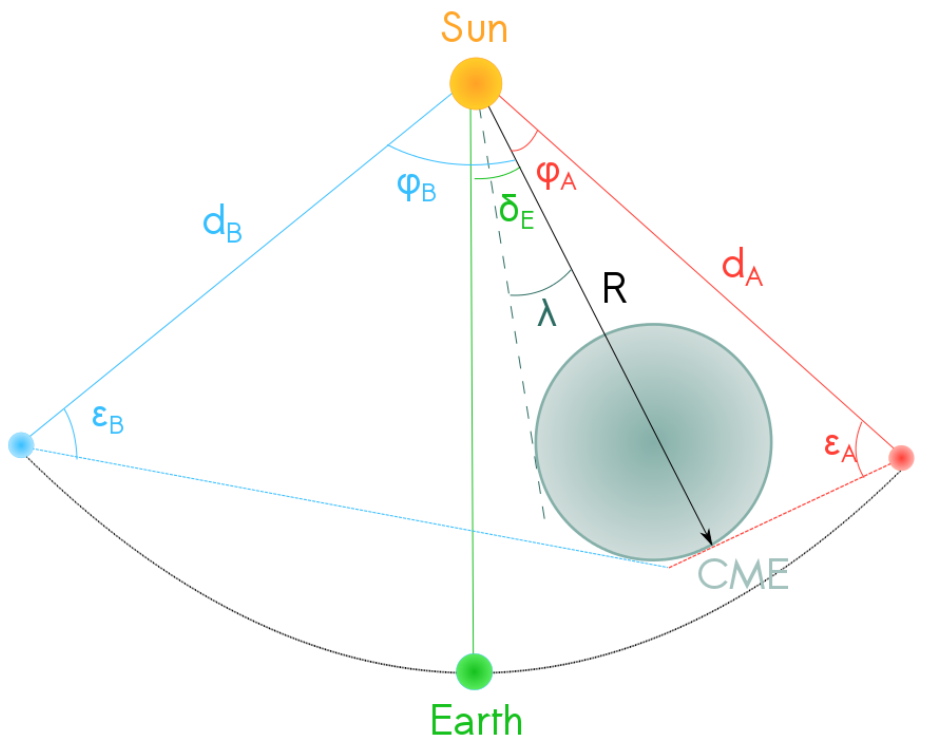

Figure 6.6.: Graphical representation of the Stereoscopic Self-Similar Expansion Model. Adapted from Davies et al. (2013).

The CME is a circle expanding outwards at constant half-width $\lambda$. While for the single spacecraft method $\lambda$ was derived as a result of the fitting to the data, in case of multipoint observations it is an input parameter. The system to solve in order to derive the CME kinematics is the following:

$$
\begin{aligned}
& R_{S S S E M}=d_{A} \frac{\left(\sin \epsilon_{A}\right)(1+\sin \lambda)}{\sin \lambda+\sin \left(\sin \epsilon_{A}+\phi_{A}\right)} \\
& R_{S S S E M}=d_{B} \frac{\left(\sin \epsilon_{B}\right)(1+\sin \lambda)}{\sin \lambda+\sin \left(\sin \epsilon_{B}+\phi_{B}\right)} \\
& \gamma_{A B}=\phi_{A}+\phi_{B} .
\end{aligned}
$$

The condition in Equation 6.10c implies that STEREO A, STEREO B and the observed CME front lie on the same plane. This restricts the applicability of the model to the analysis of the CME propagation on the Ecliptic. ${ }^{1}$ The elongation angles $\epsilon_{A}$ and $\epsilon_{B}$ are the angles formed between the Sun-spacecraft line and the tangent to the circular front originating from the spacecraft location. Therefore, as for the tangent to a sphere, there is no need to assume that the observing instruments are imaging the same exact feature. Again, the tangent to a sphere and the triangulation techniques are the limiting cases of the SSSEM, for $\lambda=90^{\circ}$ and $\lambda=0^{\circ}$, respectively.

Since the SSSEM was used in this work to determine the kinematics of propagating CMEs and shocks, an overview of how Equations 6.10 are solved will be provided.

\footnotetext{
${ }^{1}$ This statement is true for all the methods based on multipoint observations
} 
The solution procedure will be described shortly, but the discussion will include all the relations necessary for a numerical implementation of the method. The step-bystep solution is provided in Davies et al. (2013), and it involves equating Equations 6.10a and 6.10b, and substituting Equation 6.10c in the resulting identity. A quadratic equation in $\cos \phi_{A}$ can be obtained by introducing the quantities

$$
\begin{aligned}
& P=\frac{d_{B} \sin \epsilon_{B}}{d_{A} \sin \epsilon_{A}} \\
& L=\cos \left(\epsilon_{B}+\gamma\right)+P \cos \epsilon_{A} \\
& M=\sin \left(\epsilon_{B}+\gamma\right)-P \sin \epsilon_{A} \\
& N=(1-P) \sin \lambda .
\end{aligned}
$$

The system of equations 6.10 therefore has two solutions, corresponding to

$$
\tan \phi_{A}=\frac{L N \pm M \sqrt{L^{2}+M^{2}-N^{2}}}{-M N \pm L \sqrt{L^{2}+M^{2}-N^{2}}}
$$

Both solutions should be computed and the one with no physical meaning should be discarded a-posterior $i^{2}$. Once $\phi_{A}$ is computed, the height $R$ can be obtained via Equation 6.10a. Simultaneous measurements of elongation angles can be used to derive the temporal evolution of the CME heliocentric distance and the direction of propagation. The speed is derived as the numerical derivative of the height-time profile using a three point Lagrangian interpolation (Davies et al., 2013). Eruption and arrival times at a given location can be determined by fitting the $R_{S S S E M}(t)$ profile to a linear and a quadratic function. In this work arrival times were derived for the Lagrangian point $L_{1}$ , i.e. at the location of $\mathrm{ACE}$, in order to verify the arrival time predictions against insitu solar wind measurements. The linear fit to the SSSEM height-time profile assumes propagation at constant fit,

$$
R_{S S S E M}(t)=v_{S S S E M}^{F I T}\left(t-t_{0}\right)
$$

and yields $v_{S S S E M}^{F I T}$ and $t_{0}$. The quadratic fit includes the effects of acceleration/deceleration:

$$
R_{S S S E M}(t)=v_{S S S E M_{0}}^{F I T}\left(t-t_{0}\right)+\frac{1}{2} a^{F I T}\left(t-t_{0}\right)
$$

\section{Arrival Time Corrections}

In order to infer the time of arrival interplanetary CMEs, some corrections need to be applied when inverting Equations 6.13 and 6.14. These depend on the CME size and its direction of propagation with respect to the Earth-Sun line (Möstl \& Davies, 2013). Let's in fact consider Figure 6.7. When the CME hits the in-situ spacecraft, unless it propagates exactly along the Sun-Earth line, its apex will be at a distance from the Sun which is larger than 1 AU.

\footnotetext{
${ }^{2}$ This is usually quite straightforward, as in many cases one of the solutions yields negative values for the SSSEM height.
} 


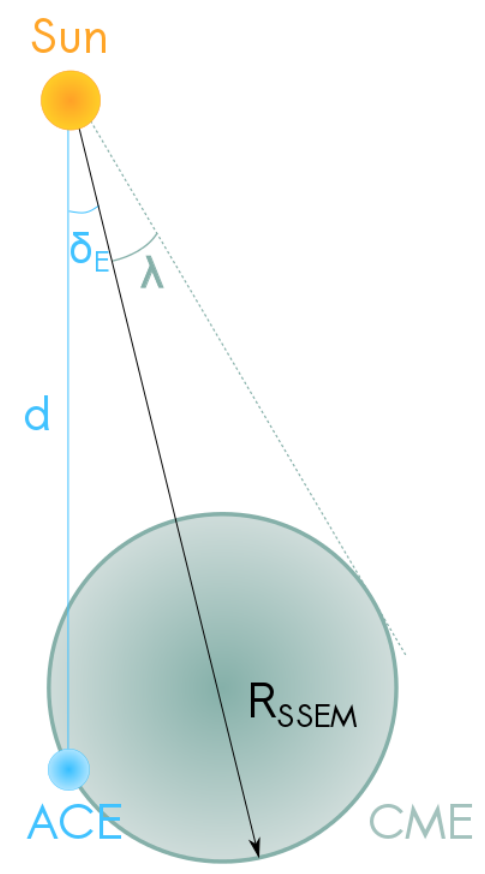

Figure 6.7.: In-situ arrival of a CME modelled with the stereoscopic self-similar expansion model. Adapted from Möstl \& Davies (2013).

It can be shown that when the CME hits the in-situ spacecraft, located at heliocentric distance $d_{A C E}$, the apex height is

$$
R_{a r r S S E M_{S}}=d_{A C E} \frac{1+\sin \lambda}{\cos \delta_{E}+\sqrt{\sin ^{2} \lambda-\sin ^{2} \delta_{E}}} .
$$

The corrections term $\frac{1+\sin \lambda}{\cos \delta_{E}+\sqrt{\sin ^{2} \lambda-\sin ^{2} \delta_{E}}}$ is zero for $\lambda=0^{\circ}$ and maximum for $\lambda=90^{\circ}$, and it increases for increasing $\delta_{E}$. When computing the in-situ CME arrival time one has to solve Equations 6.13 and 6.14 for $t$ by replacing the SSSEM height with the expression in Equation 6.15.

For the constant speed fit

$$
t_{\text {arr }}=t_{0}+\frac{R_{a r r_{S S S E M}}}{v_{S S S E M}^{F I T}}
$$

For the constant acceleration fit, on the other hand

$$
t_{\text {arr }}=t_{0}+\frac{-v_{S S S E M_{0}}+\sqrt{v_{S S S E M_{0}}^{2}+2 a^{F I T} R_{a r r_{S S E M}}}}{a} .
$$

The arrival speed also needs to be corrected. For the front not to be deformed the following must hold:

$$
\frac{R_{S S S E M}^{\text {apex }}}{d_{A C E}}=\frac{v_{S S S E M}^{\text {apex }}}{v_{\text {arr }}} .
$$

In the constant velocity fit $v_{S S S E M}$ is obtained directly from the fit itself, 


$$
v_{S S S E M}^{a p e x}=v_{S S S E M}^{F I T} .
$$

For the constant acceleration fit, instead, it is given by

$$
v_{\text {SSSEM }}^{\text {apex }}=\sqrt{v_{S S S E M_{0}}^{2}+2 a^{F I T} R_{\text {arr }_{S S S E M}}} .
$$

A thorough derivation of the above relations (for the constant velocity case) is given in Möstl \& Davies (2013).

The models presented in this chapter allow the determination of the temporal evolution of the speed, direction of propagation with respect to the Sun-Earth line, and heliocentric distance of solar wind transients from single- and multipoint white-light observations of the solar corona and inner heliosphere. Arrival time at Earth or at the location of insitu spacecraft can be inferred based on the model results. These models are a useful tool for the study of the kinematics of coronal mass ejections, as well as for space weather forecast. Their application to STEREO/SECCHI observations of CMEs in the corona and inner heliosphere will be presented in Chapter 8 


\section{Models for the Location of the Shock}



The present chapter describes the models employed for the determination of the CMEdriven shock parameters. They are based on early models for the Earth's magnetosphere subsequently modified and applied in the context of CMEs. A historical overview is provided, the validity of the presented models is discussed and some applications are shown. The last sections describe how such models can be used in combination with models for the CME kinematics (see Chapter 6) for the derivation of the interplanetary evolution of CME-driven shock parameters from remote-sensing observations of the solar corona and inner heliosphere.

Let's assume to be in the presence of a flow past an obstacle moving faster than the propagation speed of waves in the medium. In this situation a shock will form in front of the obstacle. The position of the shock is described in terms of the standoff distance, i.e. the distance between the shock and the obstacle at the stagnation point (the point where the local flow velocity is zero, see Figure 7.1). Due to mass conservation requirements the standoff distance $\Delta$ is mainly driven by the ratio between the upstream (u) and downstream (d) densities $\rho_{u}$ and $\rho_{d}$. The shock, in fact, is located at a distance from the obstacle such that the mass flow $\rho v_{n}$ is the same on both sides of the discontinuity. In other words, since at high speed the downstream to upstream mass flow ratio is governed by the density ratio (Seiff, 1962), the location of the shock is determined by the volume of plasma necessary on both sides to ensure the required

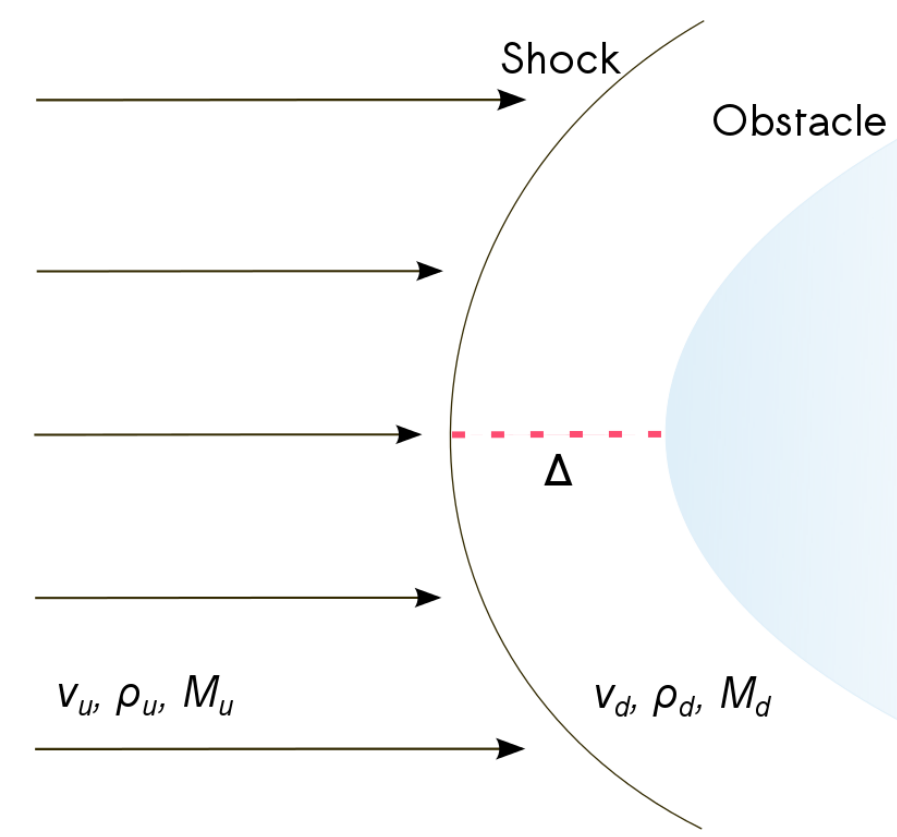

Figure 7.1.: Schematic representation of the formation of a shock in front of a blunt obstacle 
mass flux. Using a combination of theory and experiments, and for different obstacle geometries, Seiff (1962) and Spreiter et al. (1966) showed that the standoff distance $\Delta$ normalized to the size of the obstacle $D$ is a linear function of the compression ratio according to:

$$
\frac{\Delta}{D}=k \frac{\rho_{u}}{\rho_{d}}
$$

$k=0.78$ for a sphere (Seiff, 1962), whereas for an oblate obstacle $k=1.1$ (Spreiter et al., 1966). The value of the constant $k=1.1$ was determined from a combination of hydrodynamic-based calculations in the upstream sonic Mach number regime $M_{u}>5$ and experiments conducted firing a metallic model magnetosphere through argon at upstream sonic Mach number $M_{u}=4.65$ (Spreiter et al., 1966). Figure 7.2, from Spreiter et al. (1966), illustrates the successful comparison between the observed bow shock location and the model prediction. The study was conducted in order to develop models for the location and shape of the Earth's bow shock, whose distance from Earth was determined to be $R_{B S}=13 R_{E}$, with $R_{E}$ being the Earth radius.

The density ratio can be expressed by means of hydrodynamic relations as a function of $\gamma$, the adiabatic index, and the upstream Mach number, $M_{u}$ according to:

$$
\frac{\rho_{u}}{\rho_{d}}=\frac{(\gamma-1) M_{u}^{2}+2}{(\gamma+1) M_{u}^{2}}
$$

(Landau \& Lifshitz, 1959). Equation 7.1 can therefore be rewritten in terms of the upstream Mach number. For the oblate obstacle geometry investigated by Spreiter et al. (1966) we have:

$$
\frac{\Delta}{D}=1.1 \frac{(\gamma-1) M_{u}^{2}+2}{(\gamma+1) M_{u}^{2}}
$$

The dependence of the standoff distance on the ratio between upstream and downstream densities is an effect of the compressibility of the fluid: the more compressible it is, the closer the shock will stand in front of the obstacle, as a smaller volume will be necessary to satisfy the condition of mass flux conservation. On the other hand, if we look at Equation 7.3 we see that the weaker the shocks, i.e. the lower the Mach number, the further it stands away from the obstacle.

Farris \& Russell (1994) modified the right-hand side of Equation 7.3, so that it would yield the correct behaviour at low Mach numbers. The original Spreiter et al. (1966) model, in fact, yields a finite value for the standoff distance for $M_{u}=1$. Moreover, they argued that the size of the obstacle is not the relevant parameter driving the location of the shock. If that was the case, in fact, obstacles of equal sizes but with different bluntness would see the shock sitting at the same location. They suggested the radius of curvature as an alternative parameter in Equation 7.3. For an obstacle like the one used in Spreiter et al. (1966) they determined 


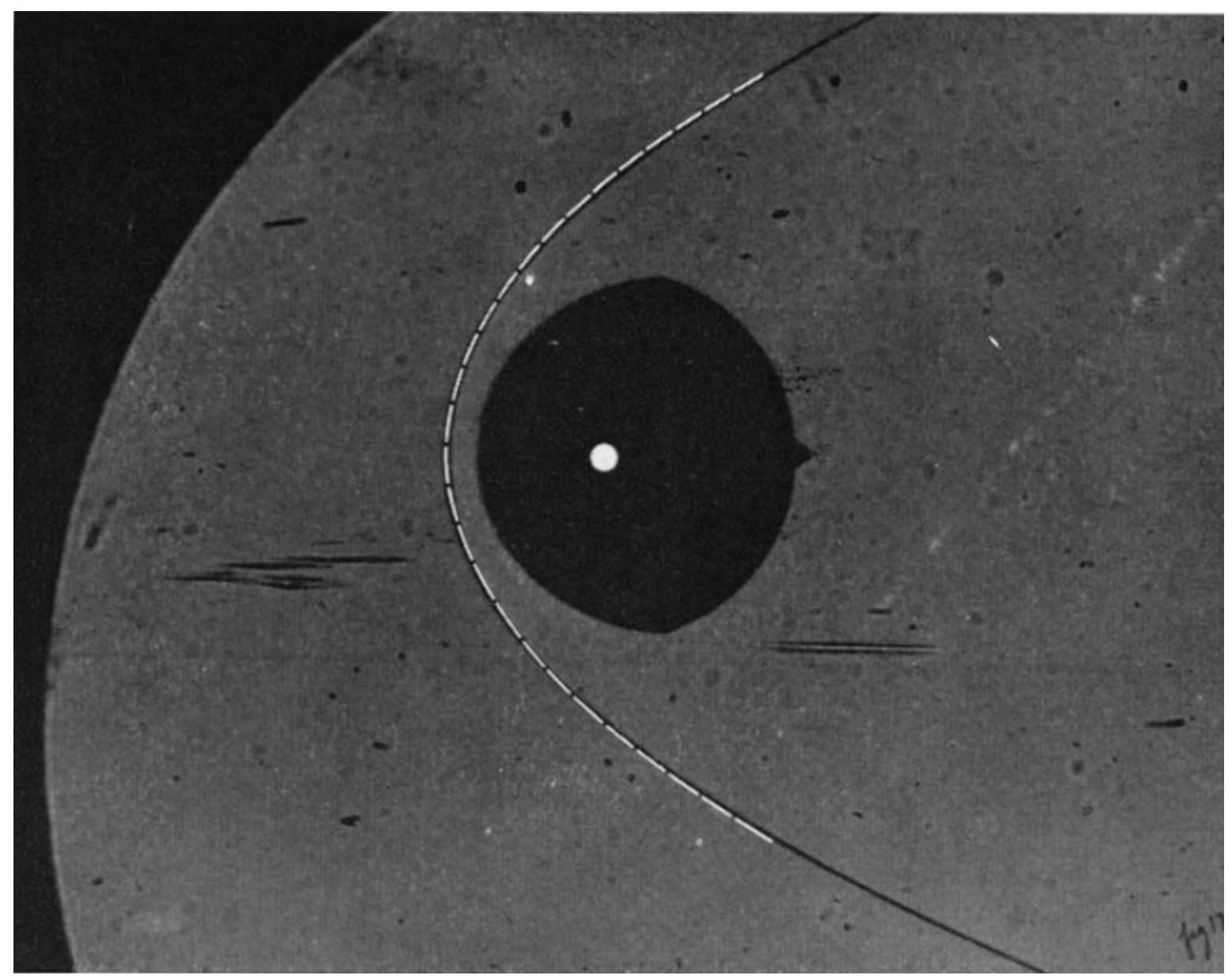

Figure 7.2.: Comparison of the shock location obtained from model and experiments. Reprinted from Spreiter et al. (1966) with the permission of AIP publishing.

$$
\begin{aligned}
\frac{\Delta}{R_{C}} & =0.81 \frac{\rho_{u}}{\rho_{d}} \\
\frac{\Delta}{R_{C}} & =0.81 \frac{(\gamma-1) M_{u}^{2}+2}{(\gamma+1)\left(M_{u}^{2}-1\right)} .
\end{aligned}
$$

The -1 term at the denominator of the right hand side in Equation $7.4 \mathrm{~b}$ ensures that the shock moves to infinity as the upstream Mach number approaches one, and thus extends the applicability of the model to the low Mach number regime.

All the equations presented so far have been developed using hydrodynamic relations ${ }^{1}$. This is clearly an approximation in all the cases in which these models are applied to magnetized systems, whose properties and evolution are described by the MHD

\footnotetext{
${ }^{1}$ To be more precise Spreiter et al. (1966) used a hydrodynamic convected-field model in which the magnetic terms in the energy and momentum equations are neglected and the magnetic field evolves according to $\boldsymbol{\nabla} \cdot \boldsymbol{B}=0$ and $\frac{\partial \boldsymbol{B}}{\partial t}=(\boldsymbol{v} \times \boldsymbol{B})$, i.e. magnetic and fluid properties decouple. The equations governing the fluid then reduce to the Navier-Stokes equations, and the magnetic field is determined via the velocity obtained as solution of the hydrodynamic equations.
} 


\section{Fast}

\section{Rotational}

\section{Slow}

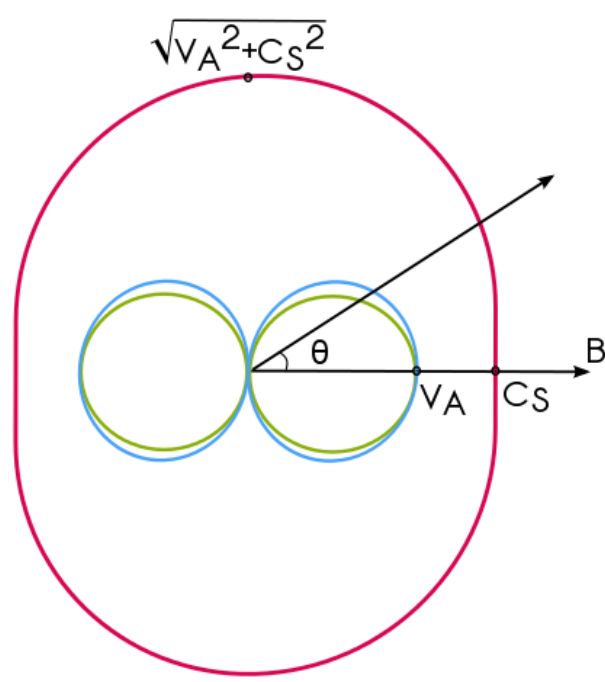

$\mathrm{V}_{\mathrm{A}}>\mathrm{CS}_{\mathrm{S}}$

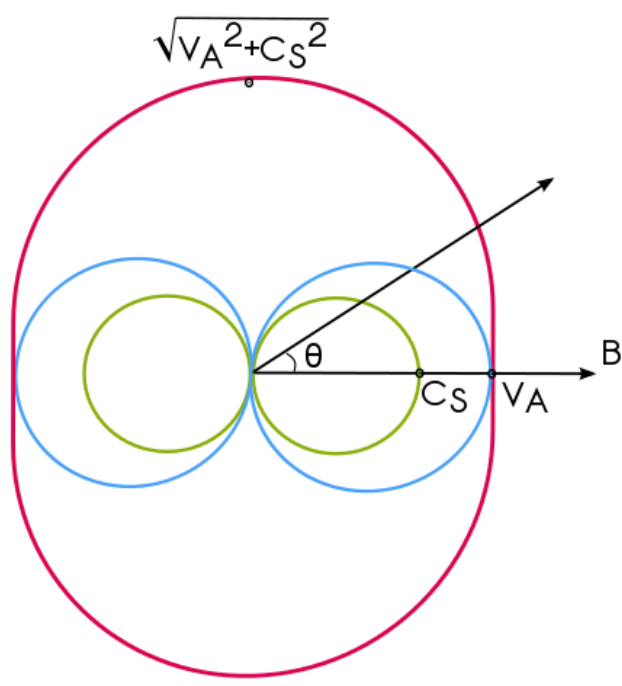

$\mathrm{V}_{\mathrm{A}}<\mathrm{CS}_{\mathrm{S}}$

Figure 7.3.: Friedrichs I diagrams for MHD waves in the cases $v_{A}>c_{s}$ and $v_{A}<c_{s}$. Adapted from Spreiter et al. (1966).

equations. Therefore, before proceeding, the regimes of validity of such approximation should be analysed. The issue has been addressed by Spreiter et al. (1966). As shown in Chapter 3, three wave modes propagates in dissipationless MHD flows. The rotational wave, propagating at speed

$$
v_{r}=v_{A} \cos \theta,
$$

and the fast and slow magnetosonic waves, propagating at speed

$$
v_{f, s}^{2}=\frac{1}{2}\left[v_{A}^{2}+c_{s}^{2} \pm \sqrt{\left(v_{A}^{2}+c_{s}^{2}\right)^{2}-4 v_{A}^{2} c_{s}^{2} \cos ^{2} \theta}\right] .
$$

The angle $\theta$ is the angle between the direction of propagation of the wave and the magnetic field, and $v_{A}$ and $c_{s}$ are the Alfvén and sound speed respectively. Friedrichs I diagrams, like the one in Figure 7.3 (see, e.g. Spreiter et al. 1966), show the dependency of the speed of each MHD wave mode on the angle $\theta$ between the direction of propagation of the waves and the magnetic field (aligned with the horizontal axis in this picture). The red, blue and green curves represent the speeds of the fast, rotational and slow waves, respectively.

As the Alfvén speed tends to zero (i.e. in the high Mach number regime) the fast wave approaches the sound wave, and the slow and rotational waves do not propagate. In 


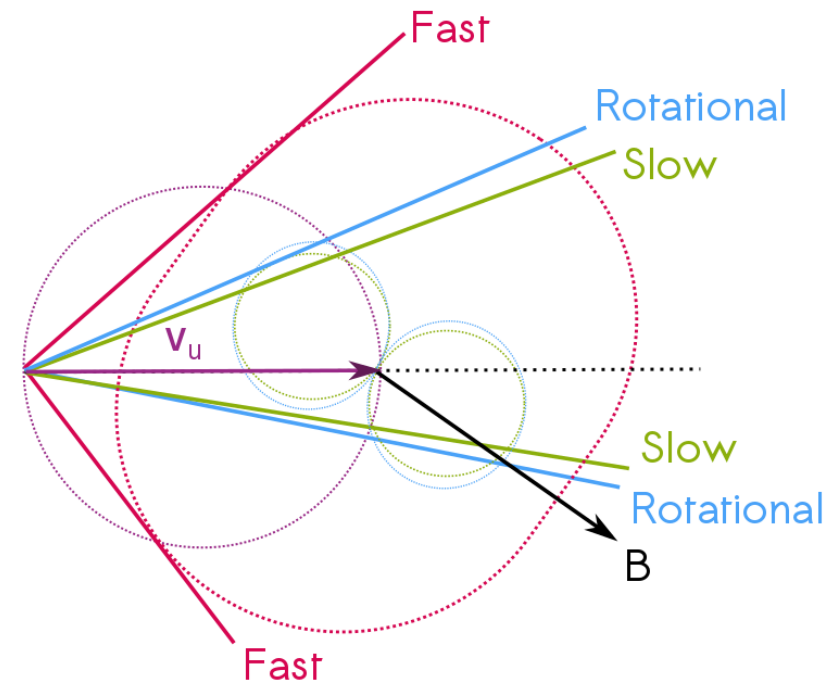

(a)
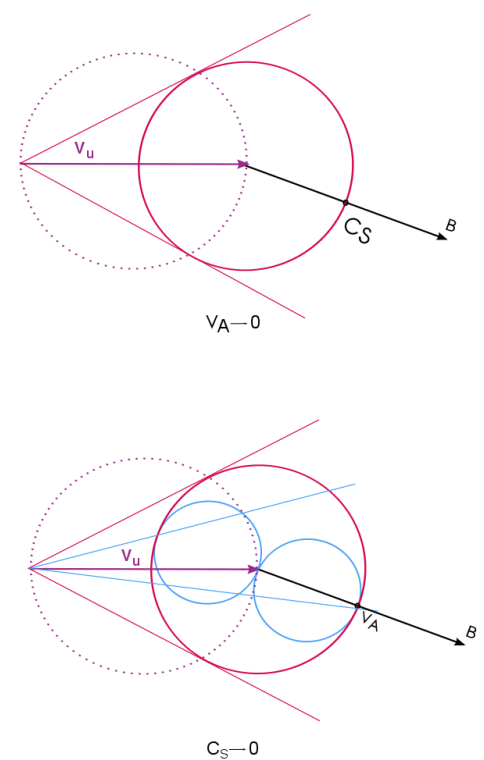

(b)

Figure 7.4.: Left panel: Friedrichs II diagrams for MHD waves in the cases $v_{A}>c_{s}$. Right panel: Friedrichs II diagrams in the limits $v_{A} \ll c_{s}$ (top) and $v_{A} \gg c_{s}$ (bottom). Adapted from Spreiter et al. (1966)

this limit the system is well described by the equations of hydrodynamics. This is quite intuitive, as the condition on the Alfvén speed implies that the magnetic field is weak ${ }^{2}$. Thus the MHD equations reduce to the Navier-Stokes equations for non-magnetized fluids. If, on the other hand the sound speed tends to zero the slow wave disappears, the fast wave propagates at the Alfvén speed, and the rotational wave retains its properties. In this case, unless the magnetic field and the velocity are parallel at all points, the use of hydrodynamic equations to describe the behaviour of MHD shocks is not fully appropriate (Spreiter \& Stahara, 2013, Spreiter et al., 1966).

However, as pointed out by Spreiter et al. (1966), the asymptotic directions of fast waves at great distance from the obstacle for $v_{A} \gg c_{s}$ tend to those of hydrodynamics, with $M=M_{A}$. This can be understood if we look at Friedrichs II diagrams (see Figure 7.4a and Spreiter et al. 1966). In these plots the origin is at the end of the upstream velocity vector $\mathbf{v}_{\mathbf{u}}$, and a circle of diameter $v_{u}$ is drawn centred at $v_{u} / 2$. A Friedrichs I diagram is overplotted, centred at the tip of $\mathbf{v}_{\mathbf{u}}$, and oriented according to the direction of the magnetic field. The Mach lines for the three MHD waves are obtained by joining the origin to the intersection between the circle centred at $v / 2$ and the respective curve in the Friedrichs I diagram. This defines the opening angle of the Mach cone $\omega$. We recall that for ordinary sound waves $\omega$ is determined by the ratio between the upstream speed and the sound speed according to $\sin \omega=\frac{c_{s}}{v_{u}}$, and that the Mach cone determines the region of influence of the perturbations induced by a

\footnotetext{
2i.e. that $B<<\sqrt{\mu_{0} \rho}$
} 
point source travelling at speed $v_{u}$. For MHD systems Spreiter \& Stahara (2013) show how the Mach angle $\omega$ varies as a function of $M_{s}, M_{A}$ and $\theta$ for specific symmetries of the shock-obstacle system. Verigin et al. (2003) determined a generalized relation to determine the Mach angle. For the purposes of this discussion we are only interested in the limits $M_{A} \ll M_{s}$ with $M_{s} \rightarrow \infty$ and $M_{s} \ll M_{A}$ with $M_{A} \rightarrow \infty^{3}$. The Friedrichs II diagrams for these limiting cases are shown respectively in the top and bottom panel of Figure 7.4b. In the former, as discussed above, the slow and rotational wave do not propagate, and the fast mode propagates at the speed of sound. In the latter, although the rotational wave still propagates, the Mach cone for the fast mode resembles the Mach cone of ordinary sound waves, but with propagation speed $v_{A}$. For this reason, as assessed by Fairfield et al. (2001), "this approach works quite well when the MHD Mach numbers are high, and it also provides a useful first approximation when Alfvén or fast magnetosonic Mach numbers are low and these Mach numbers are substituted for the gasdynamic Mach number."

\subsubsection{Using the SSSEM to determine the CME-driven shock parameters}

The models presented in the previous section have been employed to determine the location of planetary bow shocks (Fairfield et al., 2001, Farris \& Russell, 1994, Russell, 1985, Spreiter et al., 1966). Russell \& Mulligan (2002) applied the Farris \& Russell (1994) model to determine the standoff distance of a CME-driven shock at 1 AU. More recently, Gopalswamy \& Yashiro (2011), Maloney \& Gallagher (2011), Poomvises et al. (2012) exploited remote-sensing observations to determine the interplanetary standoff distance, compression ratio and Mach number via forward modelling of the CME geometry. They used respectively a conic section (and a modified conic section for the shock), a circular front and the GCS model by Thernisien et al. (2006). Gopalswamy \& Yashiro (2011) and Poomvises et al. (2012) used their result to determine the upstream interplanetary magnetic field under the assumption of low $M_{A}$ in the solar wind. A similar approach was followed also by Kim et al. (2012).

In the present work the stereoscopic self-similar expansion model (Davies et al., 2013) was applied to simultaneous STEREO A and B observations of CME and CME-driven shocks to infer the temporal evolution of the upstream Mach number and density compression according to Equations 7.4b and 7.4a. A description of the SSSEM is provided in Chapter 6. In summary, it allows the determination of the temporal evolution of the height, direction of propagation with respect to the Sun-Earth line and the speed of solar wind transients from the analysis of remote-sensing observations. The model was applied to observations of CMEs and CME-driven shocks, and their kinematics was determined separately. The temporal evolution of the standoff distance was derived as

$$
\Delta(t)=R_{S S S E M}^{S h o c k}(t)-R_{S S S E M}^{C M E}(t) .
$$

The CME radius of curvature, needed to compute the density compression ratio according to Equation $7.4 \mathrm{a}$, is derived from the SSSEM geometry as the radius of the

\footnotetext{
${ }^{3}$ i.e. $v_{A} \gg c_{s}$ with $c_{s} \rightarrow 0$ and $v_{A} \ll c_{s}$ with $v_{A} \rightarrow 0$
} 
circular front that approximates the CME leading edge:

$$
R_{C}(t)=R_{S S S E M}^{C M E}(t) \frac{\sin \lambda}{1+\sin \lambda} .
$$

Since $R_{C}=0$ for $\lambda=0^{\circ}$ the calculation of the shock parameters is restricted to the range $10^{\circ} \leq \lambda \leq 90^{\circ}$. The temporal evolution of the Mach number and the compression ratio are obtained by substituting the quantities determined according to Equations 7.7 and 7.8 in Equations 7.4a and 7.4. The linear and quadratic fits to the CME and shock height, which allow the calculation of arrival times and speeds at $L_{1}$, are also used to extrapolate the shock parameters to the same location.

\section{Accounting for the CME direction of propagation}

When employing the SSSEM for the determination of the CME-driven shock parameters, it is important to notice that Equations $7.4 \mathrm{a}$ and $7.4 \mathrm{~b}$ should be computed at the CME apex. If the angle of propagation of the CME and the shock with respect to the Ecliptic plane is large, the standoff distance and the radius of curvature of the CME computed via the SSSEM, i.e. on the Ecliptic plane, might substantially deviate from their values at the CME nose. It is then necessary to derive expressions for the standoff distance and the radius of curvature at the CME apex in order to verify the correctness of the SSSEM results. The issue has been addressed by Volpes \& Bothmer (2015) as follows.

Let's assume that the CME is a sphere, as this is the natural extension of the SSSEM geometry to 3D space. The SSSEM circle is the intersection of the sphere with the Ecliptic plane, as illustrated in Figure 7.5. The SSSEM circle along the Ecliptic plane is depicted in Figure 7.5a. The radius of the circle $r=\overline{A B}$ is

$$
r=R_{S S S E M} \frac{\sin \lambda}{1+\sin \lambda} .
$$

Figure $7.5 \mathrm{~b}$ represents the CME in the plane orthogonal to the Ecliptic and containing the vector originating at the Sun and culminating at the CME apex $\mathbf{R}_{\text {SSSEM }}=$ $\overline{O R_{S S S E M}}$ with $\alpha$ being the angle of propagation of the CME apex with respect to the Ecliptic plane. The plane slices the CME at its poles, so that the intersection between the plane in Figure 7.5b and the sphere is a circle of radius equal to the radius of the sphere $R=\overline{C H}$ :

$$
R=\frac{r}{\sin \beta} .
$$

$R$ also corresponds to the "true" CME curvature. The angle $\beta$ can be derived from trigonometric relations as (see Volpes \& Bothmer 2015)

$$
\tan \beta=\frac{r}{\left(R_{S S S E M}-r\right) \tan \alpha} .
$$

Finally, the height of the CME nose is 


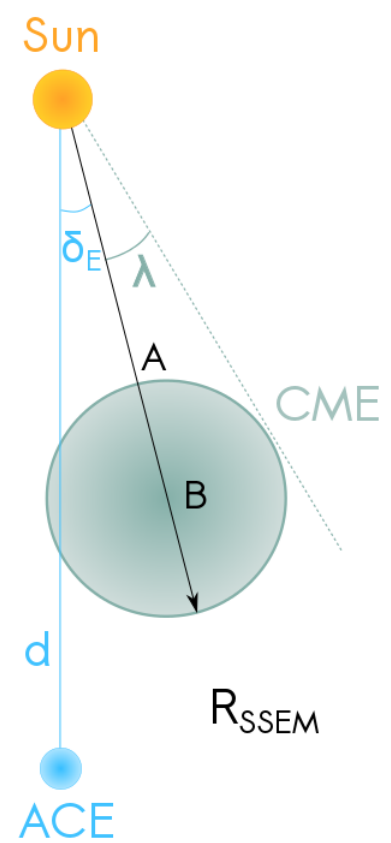

(a)

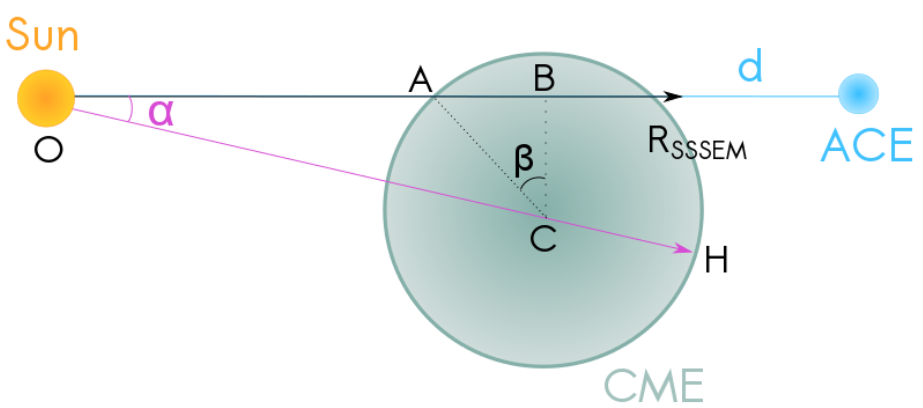

(b)

Figure 7.5.: Views of a CME modelled via the SSSEM in the Ecliptic plane (Figure 7.5a), and in a plane normal to the Ecliptic and containing the Sun-Earth line (Figure 7.5b). Adapted from Volpes \& Bothmer (2015).

$$
\overline{O H}=\frac{\left(R_{S S S E M}-r\right)}{\cos \alpha}+\frac{r}{\sin \beta} .
$$

If $\alpha$ can be determined, it is possible to assess the validity of the in-situ extrapolations of shock parameters computed via the SSSEM. In this work the latitude derived from the GCS fits is used. For a given value of $\alpha$, the error decreases for increasing CME size, i.e. for increasing $\lambda$. Larger CMEs propagating in the same direction are cut by the Ecliptic plane closer to their Equator, so that $\overline{O H}$ and $R_{S S S E M}$ do not differ too much (the same holds for the curvatures $r$ and $R$ ). On the other hand, at fixed CME size, the further away from the Ecliptic plane it propagates, the larger the error in the SSSEM standoff distance and the curvature.

The methods described in this chapter allow the derivation of the temporal evolution of the shock standoff distance, Mach number, and compression ratio by combining models for the shock locations with models for the kinematics of solar wind transients. Their application to STEREO/SECCHI observations of CMEs and CME-driven shocks will 
be discussed in Chapter 8 . 

8. Event Analysis and Results 

The present Chapter describes the results obtained via the analysis of STEREO/SECCHI observations and ACE plasma and magnetic field data for a total of 11 events. The first part will describe the event selection process and the methodology used in the analysis of the events. The second part will present the results obtained for each of the 11 events selected for this work.

The work presented in this thesis was performed within the subproject A2 of the collaborative research project SFB 963 "Astrophysical flows instabilities and turbulence"1. The SFB 963 is focused on the study of turbulence in several astrophysical environments, from galaxies to protoplanetary disks. The subproject A2 is aimed at the understanding of the processes leading to the eruption of CMEs as well as of the role of CMEs as drivers of instabilities in interplanetary space. It consists of a numerical part, carried on at the Max Planck Institute for Solar System Research, in which data-driven MHD simulations of CME onset were performed by J. Büchner and J. Skala (Skála et al., 2015), and an observational part, carried on at the University of Göttingen and presented in this work, focused on the investigation of the interplanetary evolution of CMEs and CME-driven shocks. In order to study the properties of fast CMEs and of CME-driven shocks a list of events was compiled. The list was built by looking for fast ${ }^{2}$ CMEs, capable of driving shocks in the corona and inner heliosphere, and observed simultaneously by STEREO A and B. Events which were especially attractive from a space weather perspective were also included. The initial list was subsequently expanded to increase the number of events in which a shock parameter study could be performed. The time span was restricted to the interval between 2008 and 2013: in that period, in fact, the angular separation between STEREO A and B was such that the power of stereoscopic observations could be fully exploited. Earth impacting CMEs were preferred, as they allow for a comparison to in-situ data from the ACE spacecraft. A final requirement was that the CME source region was located centrally on the solar disk. It was driven by the magnetic field extrapolations used as the initial condition for the MHD simulations of CME eruption, in order to minimize the errors due to projection effects. This last constraint was relaxed for the events that satisfied all the other criteria. Further events, like the 12 December 2008, the 19 and 23 January 2012, were included to validate the methods against literature results. The events selected according to the criteria described so far are listed in Table 8.1.

Five among the 11 events under study had distinct signatures of an interplanetary shock in both white-light observations and time-elongation plots. For the 7 June 2011

\footnotetext{
${ }^{1}$ https://www . uni-goettingen.de/en/215327.html

${ }^{2}$ CMEs from the SOHO/LASCO database (http://cdaw.gsfc.nasa.gov/CME_list/) with speeds exceeding $1000 \mathrm{~km} \mathrm{~s}^{-1}$ were selected.
} 


\begin{tabular}{|c|c|c|c|}
\hline $\begin{array}{c}\text { CME eruption } \\
\text { date }\end{array}$ & $\begin{array}{c}\text { Shock observed in } \\
\text { white-light images and j-maps }\end{array}$ & $\begin{array}{c}\text { Detected in-situ } \\
\text { by ACE }\end{array}$ & $\begin{array}{c}\text { Simulation } \\
\text { is possible }\end{array}$ \\
\hline $2008-12-12$ & $x$ & $\checkmark$ & $x$ \\
$2010-04-03$ & $\checkmark$ & $\checkmark$ & $x$ \\
$2011-06-07$ & $\checkmark$ & $x$ & $\checkmark$ \\
$2011-08-03$ & $\checkmark$ & $\checkmark$ & $\checkmark$ \\
$2011-10-22$ & $x$ & $\checkmark$ & $\checkmark$ \\
$2012-01-19$ & $x$ & $\checkmark$ & $x$ \\
$2012-01-23$ & $x$ & $\checkmark$ & $\checkmark$ \\
$2012-03-07$ & $x$ & $x$ & $\checkmark$ \\
$2012-04-23$ & $x$ & $\checkmark$ & $x$ \\
$2012-07-12$ & $\checkmark$ & $\checkmark$ & $\checkmark$ \\
$2013-03-15$ & $\checkmark$ & & \\
\hline
\end{tabular}

Table 8.1.: List of the events investigated in this work. The dates are in format YYYY-MM$\mathrm{DD}$, and refer to the date of CME eruption. For each event information is given on whether signatures of the CME-driven shocks were identified in white-light images and j-maps, and thus whether the shock parameters could be determined, whether it was detected by ACE, and if data-driven MHD simulations can be performed based on the source region location.

event signatures were identified only in STEREO B observations, whereas for the other four events the shock was detected in both STEREO A and B images and j-maps . Two of the events, the 07 June 2011 and the 23 April 2012 events, were not detected by ACE, therefore a validation of the results against in-situ data was not possible. Finally, the locations of the source regions of six of the eleven events are compatible with the requirements set by the MHD simulations.

For each event in Table 8.1 the following steps were performed:

I.) the solar source region and signatures of CME eruptions, such as flares, EUVI dimmings and waves, filament eruptions and post-eruptive arcades, were identified via the analysis of STEREO/SECCHI and SDO EUV observations of the solar disk for the date of CME eruption. The information available in the SO$\mathrm{HO} /$ LASCO halo CME database ${ }^{3}$ was also used;

II.) modelling of the CME geometry via the GCS model (see Chapter 5, Section 5.2) was performed by fitting the GCS flux rope to the morphology of the CME observed in STEREO A and B COR2 and HI1 images. When available, the COR2 modelling results included in the AFFECTS database ${ }^{4}$ were used, and the corresponding GCS flux rope was extended to the HI1 field of view. The extension was performed by keeping all the GCS parameters constant except for the apex height in order to to test the assumption of self similar expansion for the CME. For the events not available in the AFFECTS database modelling in COR2 was performed based on the observed CME morphology and extended to HI1 images;

\footnotetext{
${ }^{3}$ http://umbra.nascom.nasa.gov/lasco/observations/halo/

${ }^{4}$ http://www . affects-fp7.eu/cme-database/database.php
} 
III.) running difference images were obtained for STEREO A and B observations in the COR2, HI1 and HI2 fields of view during the interplanetary propagation of the CME. They were used to investigate the morphological properties of CMEs, to verify the presence of CME-driven shocks, and to obtain j-maps (see Chapter 4 , Section 4.3).

IV.) time-elongation measurements were performed by manually selecting points along the tracks in the $\mathrm{j}$-maps corresponding to the propagating CMEs.

V.) the derived time-elongation profiles were used as an input for the SSSEM (see Chapter 6, Section 6.2.3), which was employed in the range $0^{\circ} \leq \lambda \leq 90^{\circ}$ to determine the CME kinematics. The errors in the quantities thus obtained are computed as described in Appendix A;

VI.) the height-time profile obtained via the application of the SSSEM was fitted to a linear and a quadratic function. The first a assumes that the CME propagates at constant speed, the second assumes that the CME propagates with constant deceleration. The fits allow the prediction of arrival time and speed at $L_{1}$ as well as the CME eruption time;

VII.) the inferred eruption times were compared to EUV observations of CME onset, and arrival times and speeds were compared to solar wind data gathered at $L_{1}$ by ACE in order to verify the results.

The analysis of the interplanetary evolution of CME-driven shocks was possible for those events in which shock signatures are visible simultaneously in STEREO A and B observations as well as in time-elongation plots. For such events the steps from IV to VII were performed separately for the CME and the shock. Additionally

VIII.) the temporal evolution of standoff distance, the Mach number and the compression ratio were computed using the results obtained via the application of the SSSEM in combination with the models introduced in Chapter 7;

IX.) the linear and quadratic fits to the CME and shock SSSEM heights were used to compute the values of the shock parameters at $L_{1}$;

X.) in-situ ACE data was used to verify the extrapolations of the shock parameters. The in-situ standoff distance was calculated from the ACE data using the CME and shock crossing time and the CME crossing speed. The in-situ Mach Number and compression ratio were compared to those found in the HarvardSmithsonian Center for Astrophysics ${ }^{5}$. The authors used several methods to determine the shock parameters from in-situ data, among which the solution of the Rankine-Hugoniot equations and the coplanarity theorem. In this work the average between all the results was used for comparison.

\footnotetext{
${ }^{5}$ https://www.cfa.harvard.edu/shocks/
} 
Before presenting the results obtained with the methods described so far, it is necessary to make some remarks.

- When computing the temporal evolution of the height, direction of propagation and velocity of a solar wind transient via the SSSEM, gaps in the derived profiles can appear. This happens whenever it is not possible to simultaneously measure elongations from both STEREO A and B j-maps. Possible reasons are missing observations, difficulties in distinguishing the signatures of the solar wind transient from background structures like e.g. coronal streamers, or the presence of celestial bodies in the field of view of the imaging instruments, such as comets or the Milky Way. Liu et al. (2010b) circumvent this issue by interpolating the elongations derived from STEREO A and B observations on a common time grid. The interpolation process, however, can produce irregularities in the computed height, speed and direction of propagation profiles, which in turn are used to determine the shock standoff distance, compression ratio and Mach number. Such irregularities can be a source of additional errors in the calculation of the shock parameters. For these reasons, although attempted, the interpolation of the measured elongations was excluded from the methods employed in the present analysis.

- For all the events studied in this work arrival times and speeds at $L_{1}$ were computed and compared to ACE data. For the cases in which an independent analysis of the CME and the shock was possible, the CME and shock arrival times are determined separately for each value of the SSSEM half-width $\lambda$. The shock arrival time is compared to the time at which a sudden increase in the plasma parameters is detected in ACE data, and the CME arrival time is compared to the time at which signatures of the passage of a CME appear in ACE data (a discussion on the in-situ signature of CMEs is provided in Section 2.6).

For the events in which a separate analysis of the CME and CME-driven shock is not possible, on the other hand, only one arrival time is computed. For such events the predicted arrival time is compared to the time at which an interplanetary shock or a compression wave followed by CME signatures is detected at ACE. Such events will be referred to as ICME.

The remainder of this chapter will present the results obtained for the events of Table 8.1. Such results will be summarised and discussed in Chapter 9. 


\subsection{The 12 December $2008 \mathrm{CME}$}

\subsubsection{Solar source region}

The CME on 12 December 2008 erupted from a high latitude region on the solar disk, located at N50W15. The lifting of a prominence was observed by STEREO EUVI 304 $\AA$ between 02:00 and 04:00 UT, associated to EUVI dimming and wave propagation.
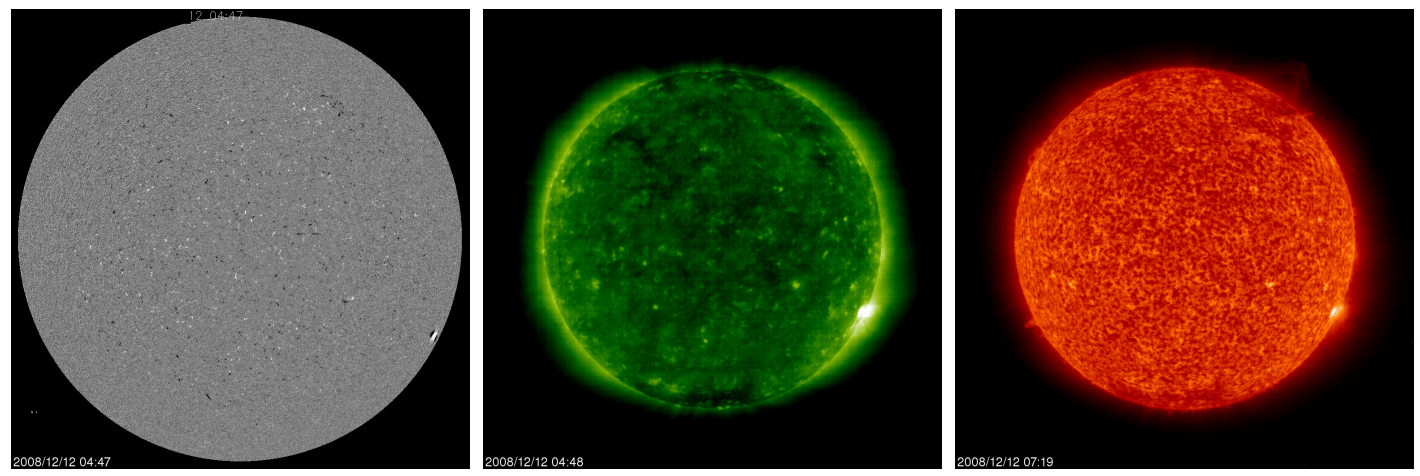

Figure 8.1.: SOHO MDI, EIT $198 \AA$ and EIT $304 \AA$ observations of the solar disk on 12 December 2008. Prominence material rising above the solar surface is seen in EIT $304 \AA$ images on the NW quadrant. Images courtesy of the NASA/ESA SOHO consortium.

Post-eruptive arcades were also observed. Figure 8.1 shows SOHO EIT $195 \AA$ EIT 304 $\AA$ and MDI observations. No large-scale active region is visible around the location where the prominence lifts off, as the event is associated to the disappearance of a quiescent filament. The CME was observed by STEREO A and B throughout its entire propagation towards Earth. At the time of observations the two spacecraft were almost in perfect quadrature, with a separation angle $\gamma_{A B}=86^{\circ}$. Composite STEREO EUVI $304 \AA$ and COR1 movies, available at http://cor1.gsfc.nasa.gov/catalog/ cme/2008/html/200812120455b.html, clearly show the CME eruption associated to the rising prominence, with its front leading the core material. The CME made its first appearance in the COR1 B field of view on 12 December 2008 at 04:35 UT and in the in the COR2 A field of view on 12 December 2008 at 04:55 UT. It crossed the COR2 $A$ and B fields of view on the same day at 07:37 UT, where the apex seems to have moved towards lower latitudes. Byrne et al. (2010) linked this effect to the coronal magnetic field configuration typical of solar minimum conditions. Statistical studies (Gopalswamy et al., 2003b) show that deflection of CMEs from high latitudes toward the equator occurs in particular during solar minimum, when the Sun's magnetic field is in a bipolar configuration. Under these conditions CME are deflected at lower latitudes either by their interaction with the magnetic field (Kilpua et al., 2009), or with the fast solar wind streaming out from coronal holes (Cremades \& Bothmer, 2004), which at times of minimum activity are mostly confined to the poles.

The heliospheric imagers HI1 and HI2 followed the evolution of the CME, which entered their fields of view respectively on 12 December 2008 at 14:49 UT and on 13 December 2009 at 14:09 UT. Figure 8.2 illustrates difference images of the CME as it propagates through the upper corona and inner heliosphere. The Earth is visible in HI2 images, the 

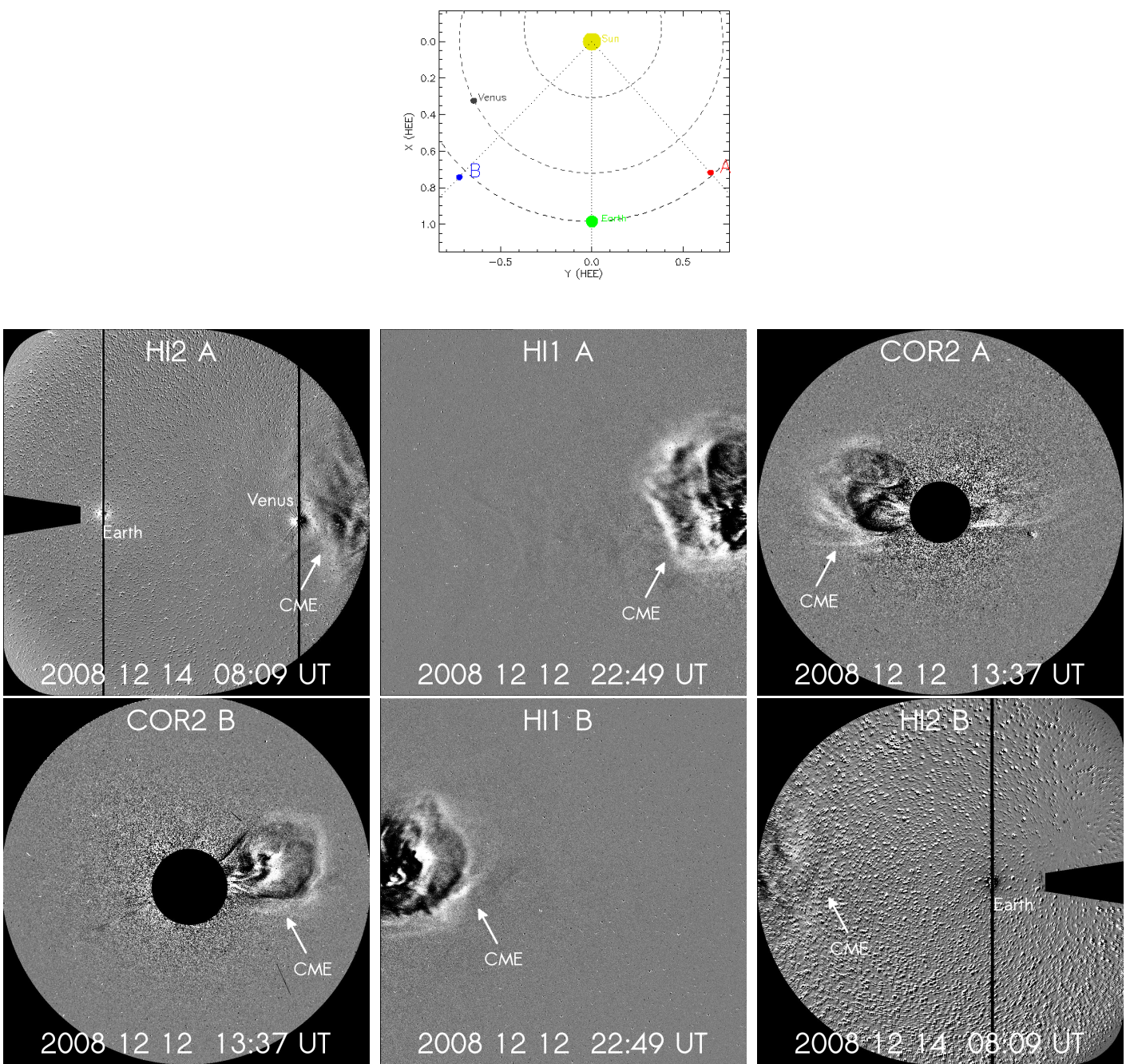

Figure 8.2.: Top panel: positions of the STEREO spacecraft on 12 December 2008. Image credit: NASA STEREO consortium. Bottom panel: difference images obtained from STERE$\mathrm{O} /$ SECCHI A and B COR2 HI1 and HI2 observations between 12 and 14 December 2008, showing subsequent phases of the CME evolution. The top and bottom panel respectively show STEREO A and B images. The Earth is visible in the HI2 images. The planet in the inner HI2 A field of view is Venus.

second planet in the HI2 A image is Venus. The event was studied by several authors, who analysed its in-situ and remote properties (Byrne et al., 2010, Davis et al., 2009, DeForest et al., 2013, Liu et al., 2010a). Here we report the results of forward and inverse modelling techniques, and a comparison with in-situ measurements.

\subsubsection{GCS modelling}

The CME was modelled via the GCS model by expanding the COR2 results available in the AFFECTS CME database to the HI1 field of view.

Table 8.2 shows the GCS parameters, the imaging instruments, and the time of obser- 

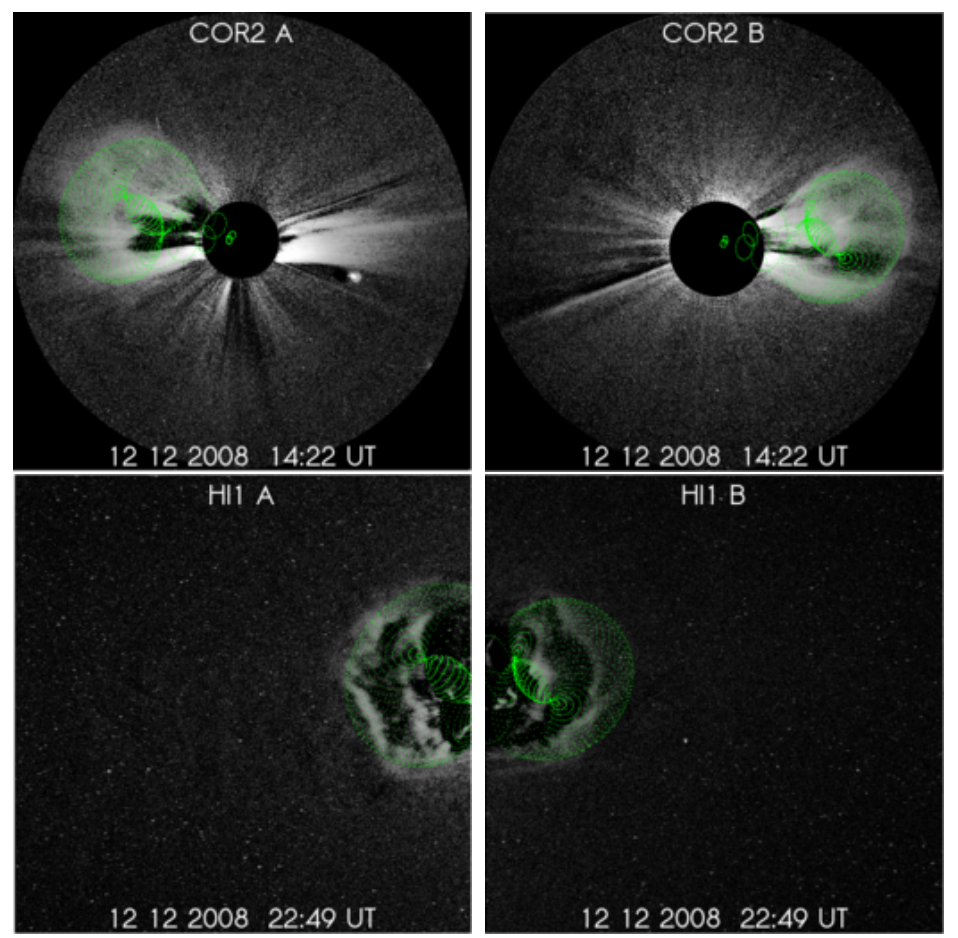

Figure 8.3.: GCS modelling for COR2 (upper panel) and HI1 (lower panels) for observations of the 12 December 2008 CME at 14:22 UT and 22:49 UT.

\begin{tabular}{|r|l|l|l|l|l|l|l|l|}
\hline Instrument & Time & Lon & Lat & Tilt & Height & $\mathrm{k}$ & $\alpha$ & $\mathrm{a}$ \\
\hline COR2 & 2008-Dec-12 14:22:00 & $70.43^{\circ}$ & $5.03^{\circ}$ & $50.87^{\circ}$ & $16.07 R_{\odot}$ & 0.27 & 10.06 & $4.33 R_{\odot}$ \\
HI1 & 2008-Dec-12 22:49:00 & $70.43^{\circ}$ & $5.03^{\circ}$ & $50.87^{\circ}$ & $44.43 R_{\odot}$ & 0.27 & 10.06 & $11.99 R_{\odot}$ \\
HI1 & 2008-Dec-13 12:49:00 & $70.43^{\circ}$ & $5.03^{\circ}$ & $50.87^{\circ}$ & $77.13 R_{\odot}$ & 0.27 & 10.06 & $20.82 R_{\odot}$ \\
\hline
\end{tabular}

Table 8.2.: GCS modelling results for the 12 December 2008 CME. The table shows the imaging instrument, the time of observations, and the GCS parameters: Carrington longitude, heliographic latitude and tilt angle of the solar source region, flux rope height $R_{G C S}$, aspect ratio $k$, half-angle $\alpha$, and radial width $a=k R_{G C S}$.

vation which were used for modelling. The CME could be fitted to the same geometry in COR2 and HI1 images, suggesting that it expanded self-similarly, as proposed by Thernisien et al. (2006). The radial width of the CME, i.e. the radius of the circular cross section $a$ at the apex ${ }^{6}$, increased from a value of $4.33 R_{\odot}$ on 12 December 2008 at 14:22 UT to more than $20 R_{\odot}$ in less than one day. A comparison between the source region latitudinal position and the location of the erupting prominence in EUV images confirms that the CME deflected towards the solar equator. This is consistent with the findings of Cremades \& Bothmer (2004). The average apex speed between the first two images is $649 \mathrm{~km} \mathrm{~s}^{-1}$, and it decreases as the CME moves outwards, with a value of $451 \mathrm{~km} \mathrm{~s}^{-1}$ between the second and the third image. Note that the averaging times are respectively 8 hours and 24 minutes and 14 hours. The average deceleration was

\footnotetext{
${ }^{6} a=k R_{G C S}$, with $k$ being the aspect ratio and $R_{G C S}$ being the apex height
} 
$-2.43 \mathrm{~m} \mathrm{~s}^{-2}$. A comparison with the SSSEM height was possible only for the second snapshot, with apex height $R_{G C S}=44 R_{\odot}$. The SSSEM yields $R_{S S S E M}=43 \pm 3 R_{\odot}$ for $\lambda=0^{\circ}$ and $R_{S S S E M}=38 \pm 3 R_{\odot}$ for $\lambda=90^{\circ}$. The values are compatible with each other, with the GCS height being higher, as expected, since the SSSEM height is computed along the Ecliptic plane.

\subsubsection{SSSEM results and in-situ comparison}

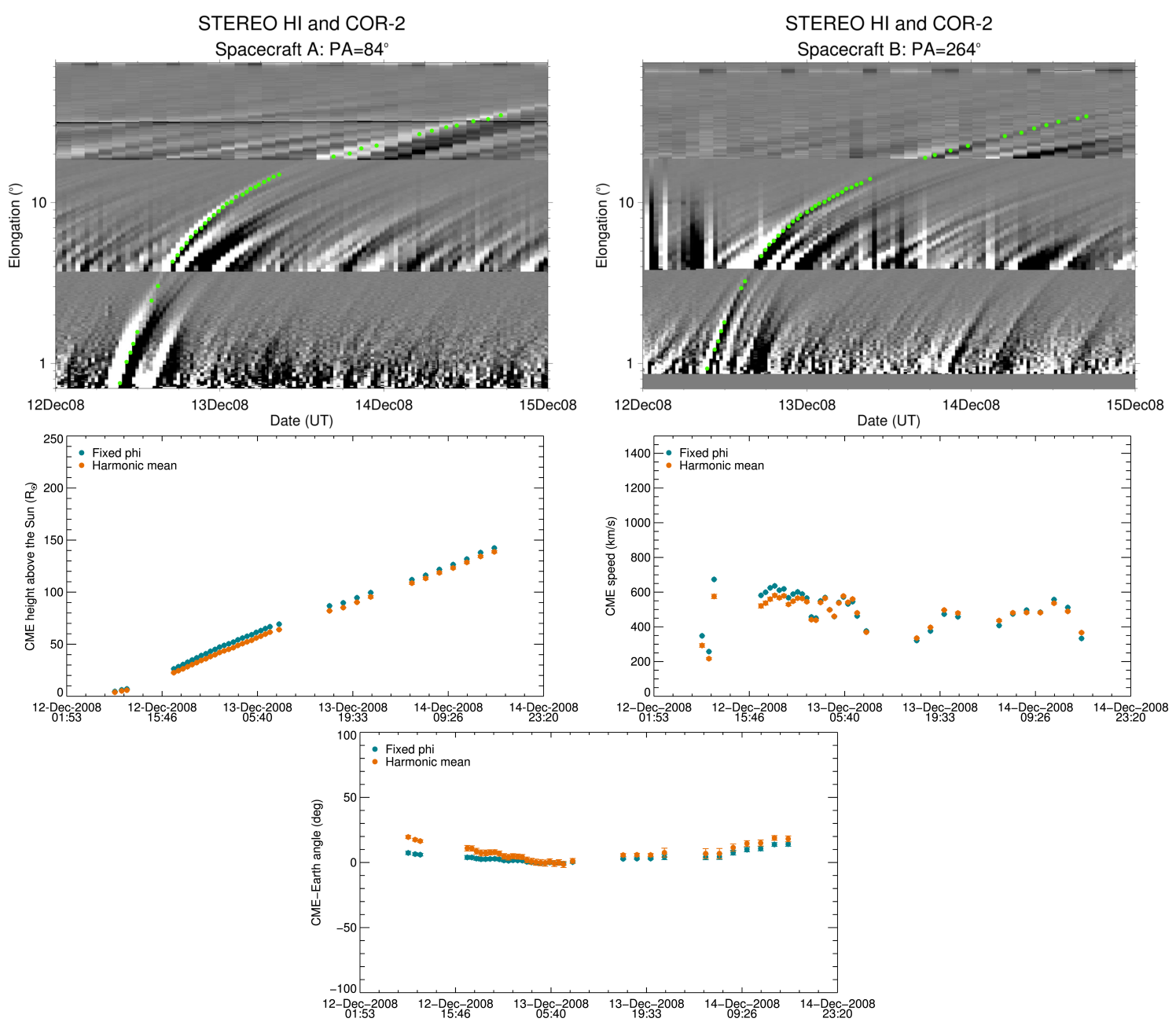

Figure 8.4.: Top panel: j-maps obtained from STEREO A (left) and B (right) observations between 12 and 15 December 2008. The green dots represent the measured elongations used to derive the CME kinematics. Bottom panel: temporal evolution of the CME height, speed and direction of propagation towards Earth obtained by applying the SSSEM for $\lambda=0^{\circ}$ and $\lambda=90^{\circ}$.

The CME kinematics was determined by applying the SSSEM (see Chapter 6) to the time-elongation profiles obtained from simultaneous STEREO j-maps . No clear shock front is visible in either difference images or j-maps, therefore only the CME propagation was investigated. The time-elongation plots for STEREO A and B COR2 
HI1 and HI2 observations along the Ecliptic plane are shown in the top panel of Figure 8.4. The green dots in the plots represent the measured time-elongation pairs. They correspond to the inner boundary of the CME leading edge in the difference images of Figure 8.2. Measurements for this event were possible up to the distances imaged by HI2. These were used to derive the temporal evolution of the CME height, direction of propagation and speed, which are shown in the bottom panel of Figure 8.4 for the SSSEM half-width $\lambda=0^{\circ}$ and $\lambda=90^{\circ}$. The average direction of propagation with respect to the Sun-Earth line was found to be $\delta_{E}=3^{\circ} \pm 3^{\circ}$ for $\lambda=0^{\circ}$ and $\delta_{E}=7^{\circ} \pm 6^{\circ}$ for $\lambda=90^{\circ}$, and it is compatible with an Earth-impacting CME. Davis et al. (2009) studied the same event performing single spacecraft fixed- $\phi$ fitting of several features of the CME. The fit was applied to STEREO A and B HI1 and HI2 images separately. The CME feature studied here corresponds to their features A1 and B1. They found $\delta_{E}=-15^{\circ} \pm 13^{\circ}$ for $\mathrm{A} 1$ and $\delta_{E}=13^{\circ} \pm 7^{\circ}$ for B1. Liu et al. (2010a) found the direction of propagation to vary with time, but to be confined within $5^{\circ}$ from the Sun-Earth line (their Feature 1 corresponds to the one tracked in this work). These results are compatible with the ones presented here within the given errors. The CME height-time profile was fitted to a linear and a quadratic function. The linear fit yields an arrival speed of $473 \mathrm{~km} \mathrm{~s}^{-1}$, higher than the one determined by Liu et al. (2010a), who obtained $363 \pm 43 \mathrm{~km} \mathrm{~s}^{-1}$. Note though that they obtained this value by averaging the speed for data points after 14 December 2008, whereas the results reported in this Section are derived by fitting the data points in HI1, between 12 December 2008 at 14:49 UT and on 13 December 2009 at 14:09 UT. Assuming that the results can be combined, since the employed techniques are the same, this could be interpreted as evidence for CME deceleration. The comparison between the arrival time determined from ACE in-situ data and the ones derived in the present work from a linear and a quadratic fit to the CME height also shows that the inclusion of deceleration improves the arrival time and speed determinations (see Table 8.3). Figure 8.5 shows ACE measurements between 15 and 18 December 2008. The purple area shows the ICME passage through the spacecraft, between 16 December 2008 at 10:20 UT and 17 December 2008 at 20:45 UT. The first density increase on 16 December 2008 at 10:20 UT corresponds to the passage of the CME leading edge (Davis et al., 2009, DeForest et al., 2013, Liu et al., 2010a). The recorded speed is about $370 \mathrm{~km} \mathrm{~s}^{-1}$. Magnetic cloud signatures are present in ACE data between 03:30 and 14:00 UT on 17 December 2008. The magnetic cloud is followed by another region of density increase, associated to the secondary bright feature observed in remote-sensing images (see Figure 8.2).

The estimated eruption and arrival times, and the arrival speed at $L_{1}$ are reported in Table 8.3 for the linear and quadratic fit to the SSSEM heights obtained for $\lambda=0^{\circ}$ and $\lambda=90^{\circ}$. The linear fit yields arrival times earlier than the observed ones, with discrepancies between 12 and 14 hours, and correspondingly faster arrival speed. The inclusion of deceleration improves the results, with arrival speeds almost coinciding with the in-situ values (333 $\mathrm{km} \mathrm{s}^{-1}$ and $361 \mathrm{~km} \mathrm{~s}^{-1}$ versus $370 \mathrm{~km} \mathrm{~s}^{-1}$ ) and arrival times which differ from the time of arrival at ACE only by 4 hours. A second degree fit to the height time profile was performed by Liu et al. (2010a), resulting in accurate arrival time predictions. Byrne et al. (2010) found that aerodynamic drag had an important role in the CME propagation. Such results were obtained by numerically 


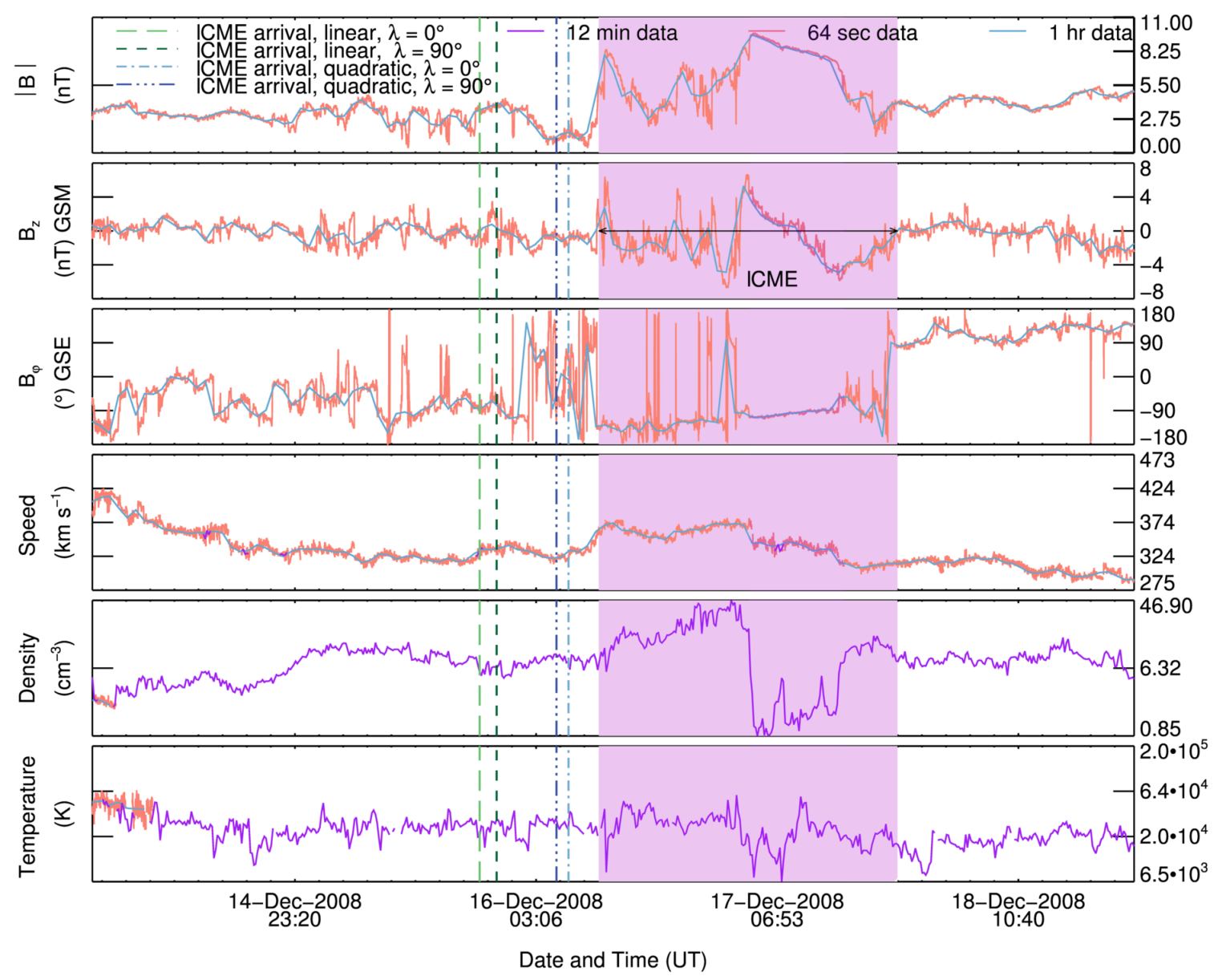

Figure 8.5.: ACE in-situ plasma and magnetic field measurements between 14 and 18 December 2008. The purple area marks the ICME passage. Overplotted are the SSSEM arrival times for $\lambda=0^{\circ}$ and $\lambda=90^{\circ}$ obtained via linear and quadratic fit to the SSSEM height.

\begin{tabular}{llll}
\hline & EUVI+ACE & 1st degree fit $\lambda=0^{\circ}$ & 1st degree fit $\lambda=90^{\circ}$ \\
\hline CME eruption time & 12-Dec-2008 02:00 & 12-Dec-2008 05:29 & 12-Dec-2008 07:24 \\
ICME arrival speed & $370 \mathrm{~km} \mathrm{~s}^{-1}$ & $473 \mathrm{~km} \mathrm{~s}^{-1}$ & $473 \mathrm{~km} \mathrm{~s}^{-1}$ \\
ICME arrival time & 16-Dec-2008 10:20 & 15-Dec-2008 20:36 & 15-Dec-2008 22:34 \\
\hline & EUVI+ACE & 2nd degree fit $\lambda=0^{\circ}$ & 2nd degree fit $\lambda=90^{\circ}$ \\
\hline CME eruption time & 12-Dec-2008 02:00 & 12-Dec-2008 04:43 & 12-Dec-2008 08:23 \\
ICME arrival speed & 370 $\mathrm{km} \mathrm{s}^{-1}$ & 333 $\mathrm{km} \mathrm{s}^{-1}$ & 361 $\mathrm{km} \mathrm{s}^{-1}$ \\
ICME arrival time & 16-Dec-2008 10:20 & 16-Dec-2008 06:50 & 16-Dec-2008 06:05 \\
\hline
\end{tabular}

Table 8.3.: Observed CME eruption time, arrival time and speed at $L_{1}$ compared to those obtained via a linear (top panel) and quadratic (bottom panel) fit to the CME height-time profile derived by applying the SSSEM for $\lambda=0^{\circ}$ and $\lambda=90^{\circ}$ for the 12 December 2008 event.

integrating the equations that determine the evolution of the CME speed under the action of the drag force and combining the results to the observed CME speeds. This 
confirms the results on the CME kinematics presented in this section. Davis et al. (2009) reported that an increase in the horizontal component of the terrestrial magnetic field was detected about 1 hour after the CME passage at $L_{1}$, resulting from compression of the day-side magnetosphere. Additionally modest sub-storms were associated to the reversal of the IMF $B_{z}$ within the magnetic cloud, causing an increase in the Ap geomagnetic index from an initial value of 4-6 to a value of 12 .

The 12 December 2008 event originated from the lift-off of a high-latitude quiescent filament observed between 02:00 and 04:00 UT. The CME was associated with EUV dimmings, a EUV wave, and post eruptive loops.

GCS modelling shows that the CME expanded self similarly, and that its speed decreased as it propagated away from the Sun. The GCS results for the height of the CME apex are compatible with the SSSEM heights.

The SSSEM yields similar height, speed and direction of propagation profiles for both $\lambda=0^{\circ}$ and $\lambda=90^{\circ}$. The SSSEM direction of propagation is indicative of propagation along the Sun-Earth line. Arrival times and speeds at $L_{1}$ were inferred by fitting the SSSEM height-time profile to a linear and a quadratic function. The comparison with $A C E$ data shows that the best prediction corresponds to the case $\lambda=90^{\circ}$ and propagation at constant deceleration, for which the estimated and observed arrival times are less than 4 hours apart, and the error in the arrival speed is of the order of $10 \mathrm{~km} \mathrm{~s}^{-1}$. Similar but slightly less accurate results are obtained for $\lambda=0^{\circ}$ in the case of constant deceleration, for which the errors in the predicted arrival time and speed are respectively 4.5 hours and $37 \mathrm{~km} \mathrm{~s}^{-1}$. 


\subsection{The 03 April $2010 \mathrm{CME}$}

\subsubsection{Solar source region}

The event analysed in this section erupted on 03 April 2010 from NOAA AR 11059 located at S25W03 on the solar disk. An in-depth analysis of the CME and the CMEdriven shock can be found in Volpes \& Bothmer (2015). The event was also studied by Möstl et al. (2010) and Liu et al. (2011), with results compatible to the ones presented here.

Solar signatures of the event were a B7.4 flare detected by the GOES spacecraft at
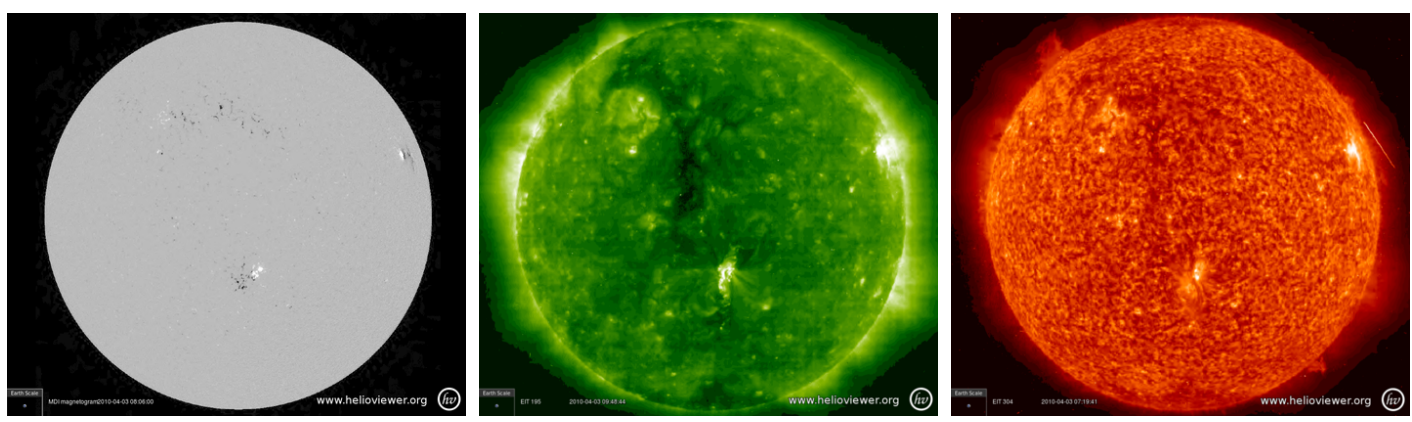

Figure 8.6.: SOHO MDI, EUVI $193 \AA$ and EUVI $304 \AA$ observations of the solar disk on 03 April 2010. Images courtesy of the NASA SDO consortium.

09:04 UT, the lift-off of a prominence, EUV dimmings and a EUV wave propagating in the SW direction. Post eruptive loops are observed in SDO EUV images.

The angular separations between each of the STEREO A and B spacecraft and Earth on 03 April 2010 were $67^{\circ}$ and $71^{\circ}$ respectively. The source region is therefore seen on the East limb from the STEREO A perspective and on the West limb from the location of STEREO B. The event entered the COR2 field of view at 09:54 UT. HI1 images of the events are available from 12:09 UT and HI2 A first observed the event at 00:09 UT on 04 April 2010. High levels of noise are present in HI2 B images due to the presence of the Milky Way in the field of view of the instrument. As a consequence stereoscopic observations of the 03 April $2010 \mathrm{CME}$ are only available to $24^{\circ}$ elongation, corresponding to the outer edge of the HI1 field of view. Figure 8.7 shows difference images of the event at different stages of its evolution. A faint precursor is seen in COR2 and HI1 images, and is interpreted as the signature of a CME-driven shock. Since stereoscopic observations of the CME and the CME-driven shock are available over a sufficiently large spatial range, the kinematic of the CME and the shock could be derived separately. The standoff distance of the shock, the upstream Mach number and the density compression ratio can be derived via the application of the models described in Chapter 7. These results are described in the rest of this section.

\subsubsection{GCS modelling}

The event was fitted with the GCS geometry in order to determine whether it expanded self-similarly. The COR2 fitting was extended to HI1 images by keeping all the param- 

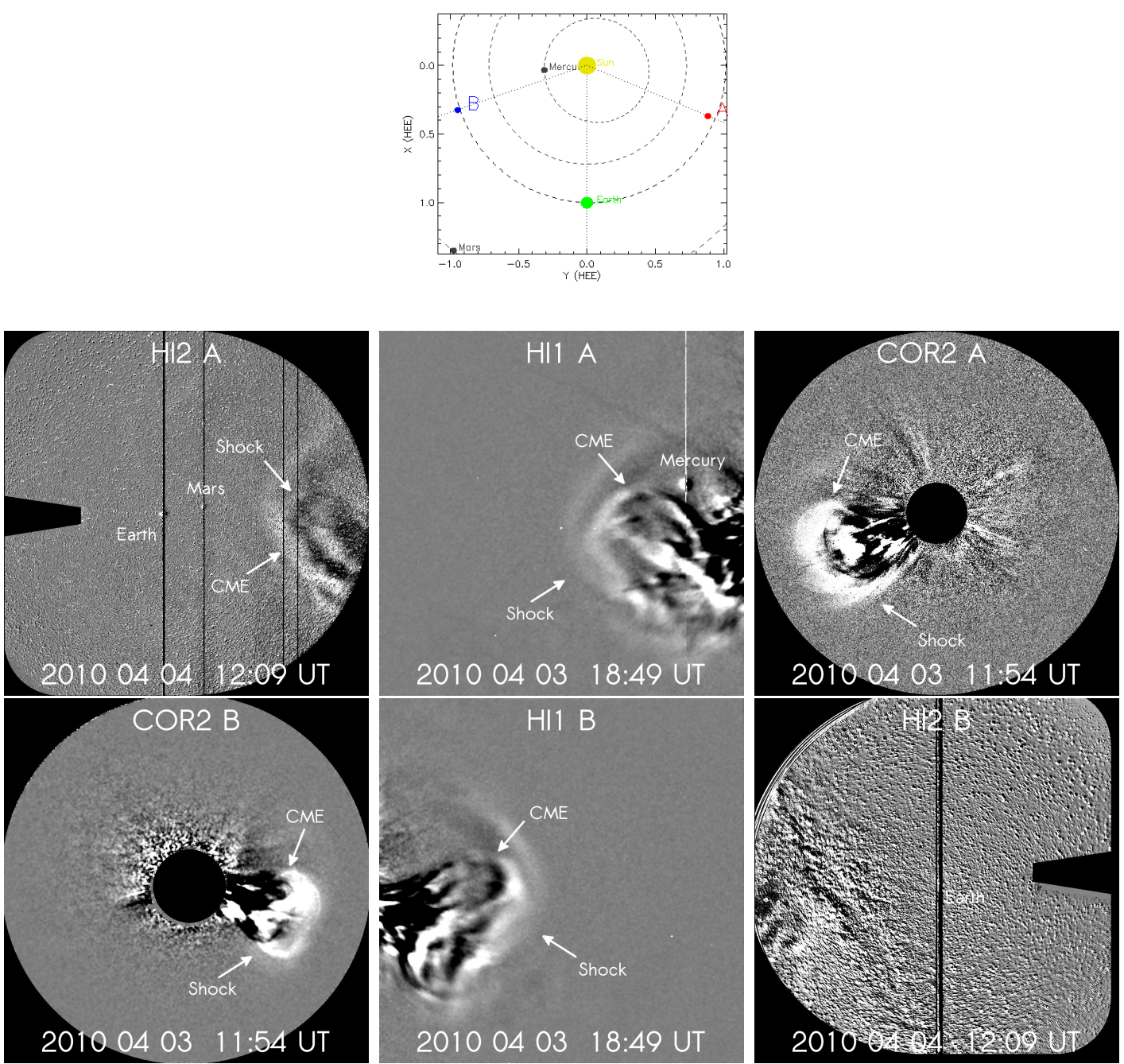

Figure 8.7.: Top panel: positions of the STEREO spacecraft on 03 April 2010. Image credit: NASA STEREO consortium. Bottom panel: difference images obtained from STEREO/SECCHI A (top panel) and B (bottom panel) COR2 HI1 and HI2 observations, showing subsequent phases of the CME evolution for the 03 April 2010 event.

eters fixed except for the CME height. The results are shown in Figure 8.8 and listed in Table 8.4.

The geometry of the CME is preserved as it expands to larger heliocentric distances. The GCS heights, $13 R_{\odot}$ at 12:08 UT, $42 R_{\odot}$ at 17:29 UT, and $59 R_{\odot}$ at 20:49 UT compare well to the results obtained via an application of the SSSEM. These yield approximately $11 \pm 1 R_{\odot}$ at 11:54 UT, $42 \pm 1 R_{\odot}$ at 17:29 UT, and $59 \pm 1 R_{\odot}$ at 20:49 UT for $\lambda=0^{\circ}$ and $11 \pm 1 R_{\odot}$ at 11:54 UT, $42 \pm 1 R_{\odot}$ at 17:29 UT, and $58 \pm 1 R_{\odot}$ at 20:49 UT for $\lambda=90^{\circ}$. The average speed between 12:08 UT and 17:29 UT is $1038 \mathrm{~km} \mathrm{~s}^{-1}$, and it decreases to a value of $1003 \mathrm{~km} \mathrm{~s}^{-1}$ between 17:29 UT and 20:49 UT, when the CME is observed in the HI1 field of view. The average deceleration between 12:08 UT and 20:49 UT was $-1.13 \mathrm{~m} \mathrm{~s}^{/ 2}$. The SSSEM speed shows a similar profile, decreasing as the CME moves from the COR2 to the HI1 field of view, but the values are some- 

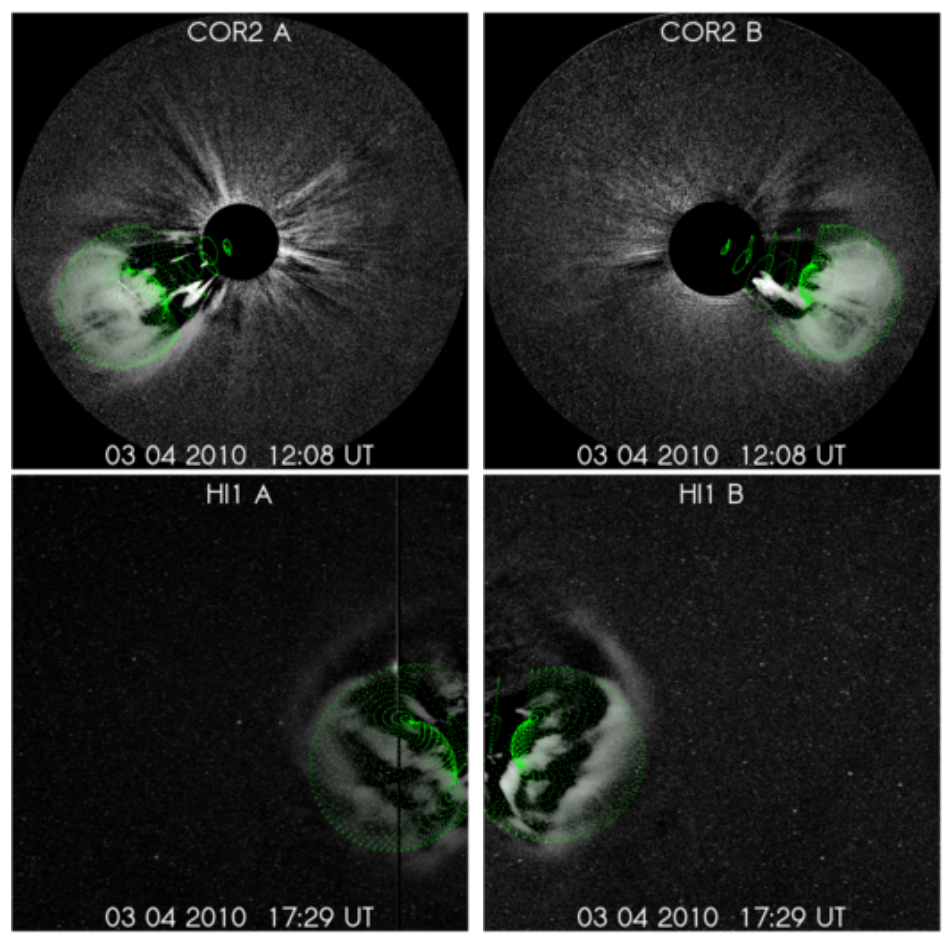

Figure 8.8.: GCS modelling for STEREO/SECCHI COR2 (upper panel) and HI1 (lower panels) observations of the 03 April $2010 \mathrm{CME}$ at 12:08 UT and 17:29 UT.

\begin{tabular}{|r|l|l|l|l|l|l|l|l|}
\hline Instrument & Time & Lon & Lat & Tilt & Height & $\mathrm{k}$ & $\alpha$ & $\mathrm{a}$ \\
\hline COR2 & 2010-Apr-03 12:08:00 & $259.38^{\circ}$ & $-23.47^{\circ}$ & $55.91^{\circ}$ & $13.64 R_{\odot}$ & 0.35 & 13.14 & $4.77 R_{\odot}$ \\
HI1 & 2010-Apr-03 17:29:00 & $259.38^{\circ}$ & $-23.47^{\circ}$ & $55.91^{\circ}$ & $42.40 R_{\odot}$ & 0.33 & 13.14 & $14.84 R_{\odot}$ \\
HI1 & 2010-Apr-03 20:49:00 & $259.38^{\circ}$ & $-23.47^{\circ}$ & $55.91^{\circ}$ & $59.71 R_{\odot}$ & 0.33 & 13.14 & $20.89 R_{\odot}$ \\
\hline
\end{tabular}

Table 8.4.: GCS modelling results for the 03 April 2010 CME. The table shows the imaging instrument, the time of observations, and the GCS parameters: Carrington longitude, heliographic latitude and tilt angle of the solar source region, flux rope height $R_{G C S}$, aspect ratio $k$, half-angle $\alpha$, and radial width $a=k R_{G C S}$.

what higher than the GCS ones. A detailed description of the SSSEM results will be given in the following Section.

\subsubsection{SSSEM results}

The time-elongation plots obtained from STEREO A and B observations between 02 and 07 April 2010 are shown in Figure 8.9. The track appearing after 03 April 2010 corresponds to the event under study. A fainter track preceding the bright CME signature is visible in the elongation range observed by HI1 and it corresponds to the passage of the CME-driven shock in the instrument's field of view. The time-elongation profiles for the CME and the shock are shown in Figure 8.9. The green and red dots correspond to time-elongation measurements performed respectively for the CME and the shock. Such measurements allow the independent determination of the CME and 

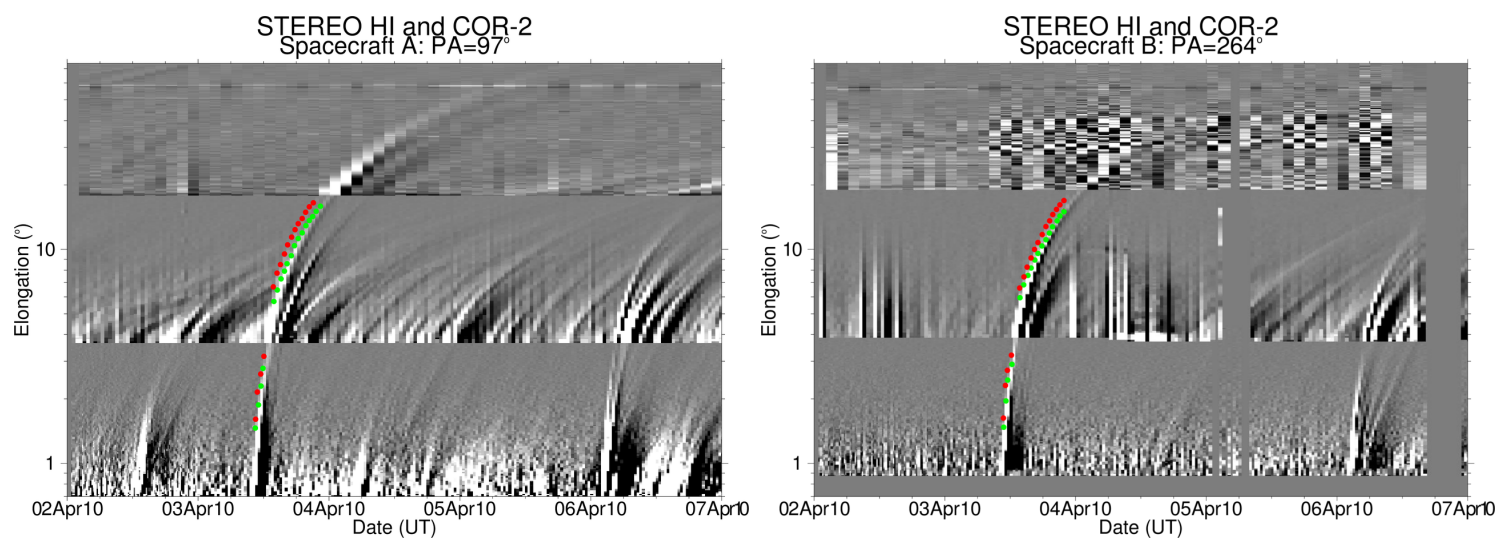

Figure 8.9.: Top panel: j-maps obtained from STEREO A (left) and B (right) observations between 02 and 07 April 2010. The red dots represent the time-elongation measurements for the shock, and the green dots are the time-elongation pairs measured for the CME.

shock kinematics via the application of the SSSEM. The results are presented in the remainder of the section.

\section{CME}

Figure 8.10 shows the temporal evolution of the CME distance from the Sun, speed, and direction of propagation with respect to the Sun-Earth line for $\lambda=0^{\circ}$ (orange dots) and $\lambda=90^{\circ}$ (blue dots). The average CME direction of propagation was found to be $\delta_{E}=$
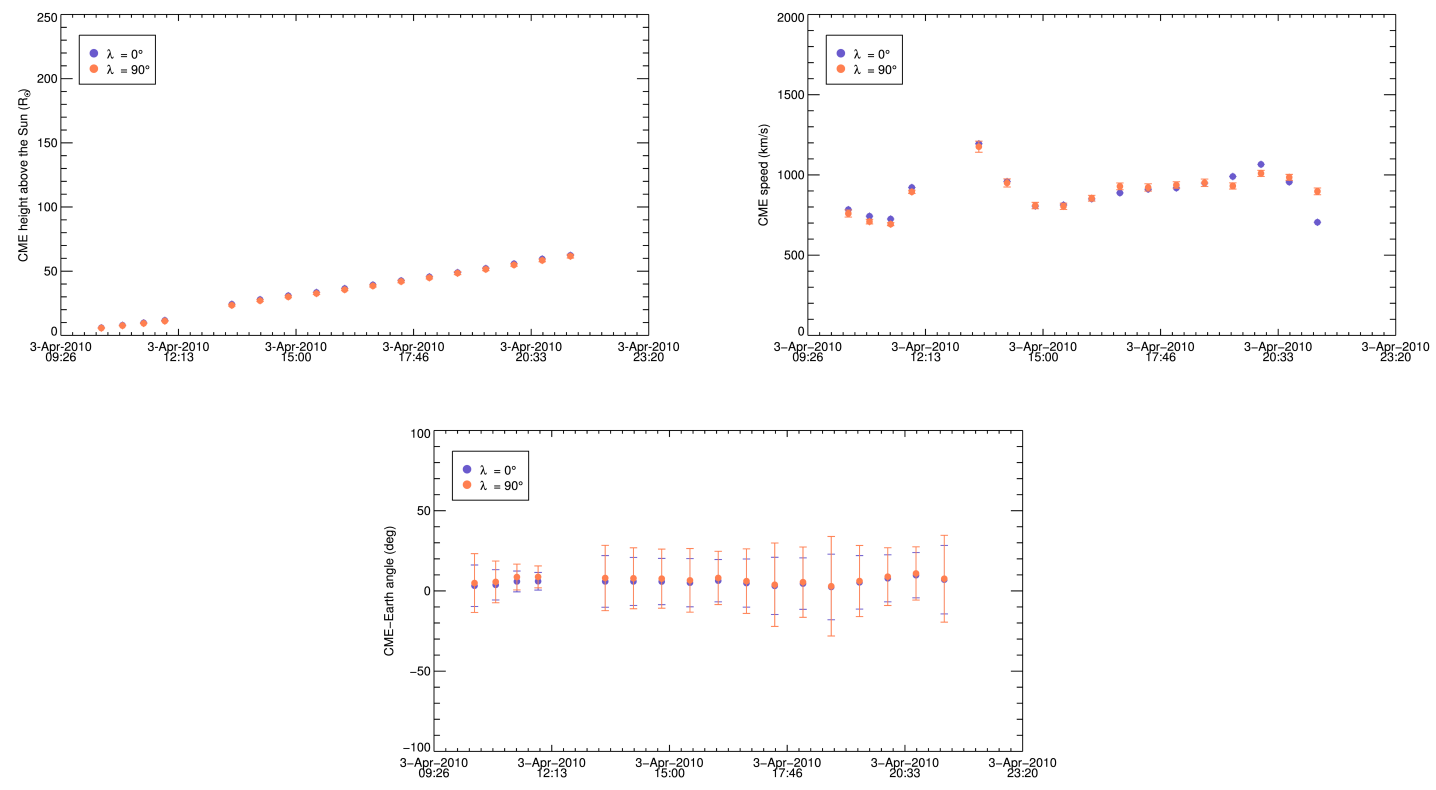

Figure 8.10.: Time evolution of the CME height, speed and direction of propagation towards Earth obtained by applying the SSSEM for $\lambda=0^{\circ}$ and $\lambda=90^{\circ}$ to STEREO/SECCHI observations of the 03 April 2010 event. 
$5^{\circ} \pm 2^{\circ}$ for $\lambda=0^{\circ}$ and $\delta_{E}=6 \pm 2$ for $\lambda=90^{\circ}$. These results are compatible with radial propagation from the CME source region, located at $W 03^{\circ}$ latitude on the solar disk. It is important to remember that when applying the SSSEM to STEREO observations we are only focusing on the propagation of solar wind transients along the Ecliptic plane. Radial propagation, in this context, should be interpreted as propagation at constant longitude. The results presented here for the CME direction are in agreement with the values determined by Möstl et al. (2010). They found $\delta_{E}=9^{\circ}$ for $\lambda=0^{\circ}$ and $\delta_{E}=-5^{\circ}$ for $\lambda=90^{\circ}$ by means of single spacecraft fitting methods, and $\delta_{E}=0^{\circ} \pm 2^{\circ}$ from GCS modelling. The CME speed seems to increase in the early phases of propagation, before decreasing towards a roughly constant profile for both values of the SSSEM half-width.

\section{Shock}

The results obtained for the shock kinematics are shown in Figure 8.11. The plots show the temporal evolution of the shock heliocentric distance, speed, and direction of propagation with respect to the Sun-Earth line. The average direction of propagation, $\delta_{E}=1^{\circ} \pm 4^{\circ}$ for both $\lambda=0^{\circ}$ and $\lambda=90^{\circ}$, is again compatible with propagation towards Earth. The speed profile, similarly to what found for the CME, shows an increase in the early phase of propagation followed by a decrease towards a rather constant value. Again, very similar results are obtained for all values of the SSSEM half width $\lambda$.
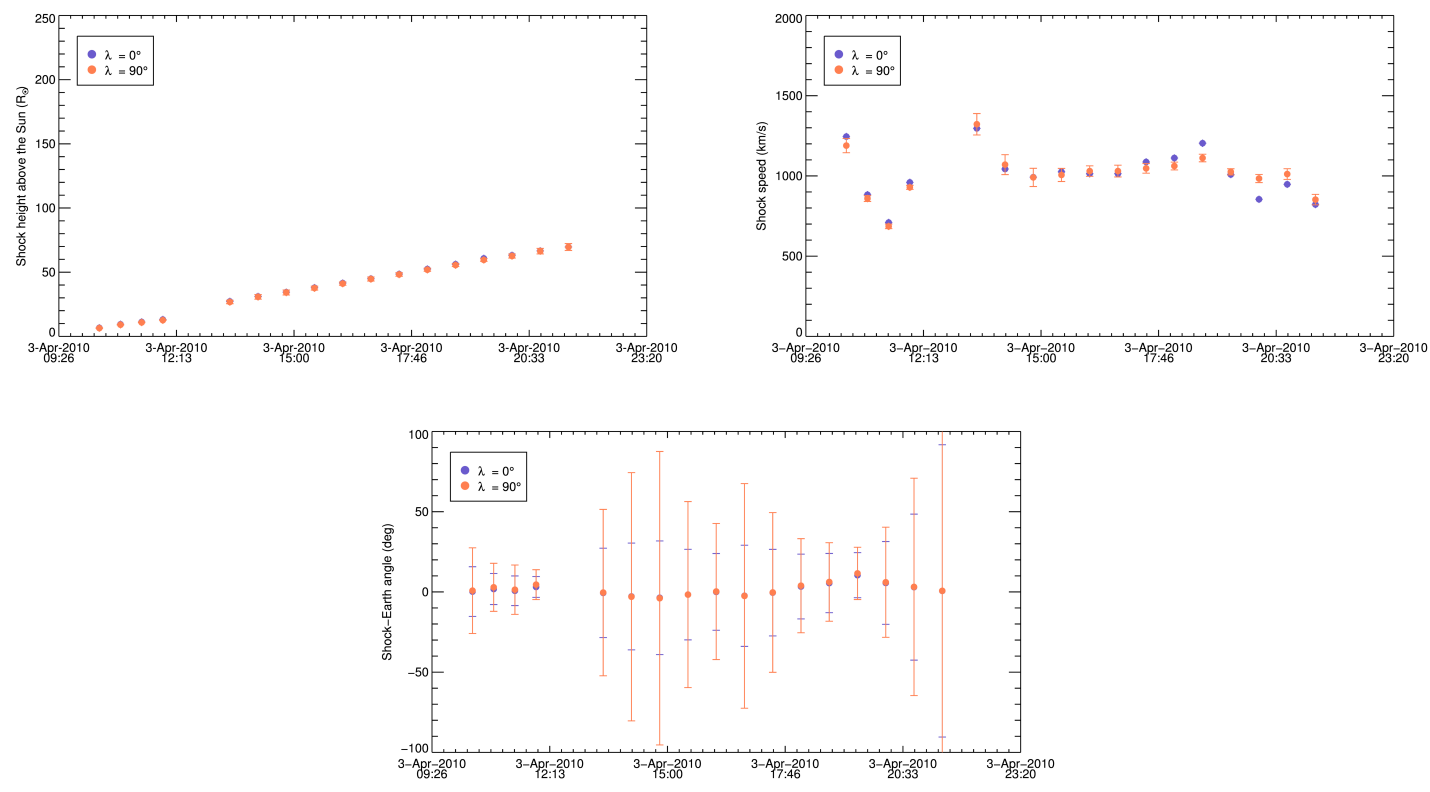

Figure 8.11.: Time evolution of the shock height, speed and direction of propagation towards Earth obtained by applying the SSSEM for $\lambda=0^{\circ}$ and $\lambda=90^{\circ}$ for the 03 April 2010 event. 


\subsubsection{CME-driven shock parameters}

The CME and shock SSSEM heights were used to determine the temporal evolution of the shock standoff distance. The CME curvature can directly be computed for each value of $\lambda$ from its heliocentric distance, as discussed in in Chapter 7, Section 7.0.4. The compression ratio and the Mach number can be derived from these values using Equation 7.4a and Equation 7.4b. Figure 8.12 shows the results for $\lambda=10^{\circ}$ and $\lambda=90^{\circ}$. The case $\lambda=0^{\circ}$ is not included, as it corresponds to zero CME curvature. This, in turn, leads to singularities in the determination of the shock parameters. The standoff distance is found to increase rather regularly with time, in agreement with STEREO/SECCHI observations, in which the shock-CME distance progressively increases during their propagation. The radius of curvature for the case $\lambda=10^{\circ}$ is by definition smaller than the one obtained for $\lambda=90^{\circ}$. Both the compression ratio and the Mach number do not seem to vary substantially with time. Similar results, but for a different event, were found by Maloney \& Gallagher (2011). The authors determined the shock parameters by combining forward geometrical modelling of the CME and shock front with the models for the shock standoff distance employed in this work. They also found that the Mach number and the compression ratio were roughly constant in time. For $\lambda=10^{\circ}$ the compression ratio is non-physically below one ${ }^{7}$. In this case it is likely that the SSSEM radius of curvature underestimates the real value. The time profiles of the shock parameters were extrapolated to $L_{1}$ for a comparison with ACE in-situ data. The results are discussed in the next Section.
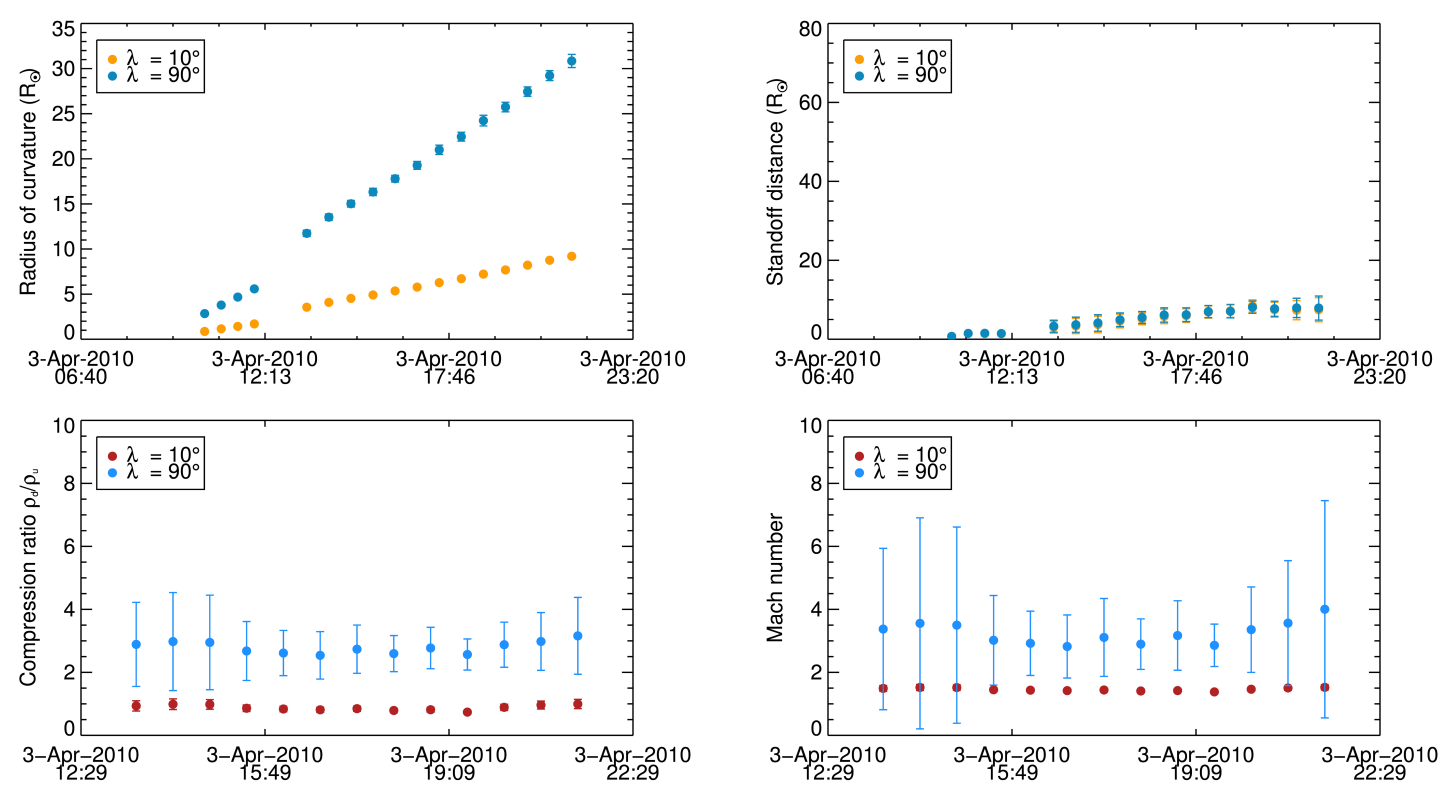

Figure 8.12.: Time evolution of the CME radius of curvature (top left), the shock standoff distance (top right), the compression ratio (bottom left) and the Mach number (bottom right) obtained by applying the SSSEM for $\lambda=10^{\circ}$ and $\lambda=90^{\circ}$ to STEREO/SECCHI observations of the 03 April 2010 event.

\footnotetext{
${ }^{7} \mathrm{~A}$ compression ratio smaller than one is not compatible with the presence of a compressive shock.
} 


\subsubsection{In-situ comparison}

In order to validate the SSSEM results, as well as the results obtained for the shock parameters, a comparison to observations is needed. ACE data was used for this purpose. The CME and shock height profiles were fitted to a first and a second degree function in order to infer arrival times at $L_{1}$. The fits yield also the arrival speed and eruption times, i.e. the time at which the SSSEM height is zero. These can be compared to the observed in-situ CME and shock arrival times ans to the time of CME onset as derived from EUV images. Figure 8.13 illustrates ACE measurements of the magnetic field strength and its components, as well as of the proton speed, density, and temperature between 04 and 07 April 2010. The vertical dashed lines indicate the CME and shock arrival as determined from the quadratic fit to the SSSEM heights. The observations show a sudden increase in the plasma parameters, corresponding to the passage of an interplanetary shock across the ACE spacecraft on 05 April 2010 at 08:00 UT. The shock is followed by the CME, which reached the location of ACE at 12:00 UT. The

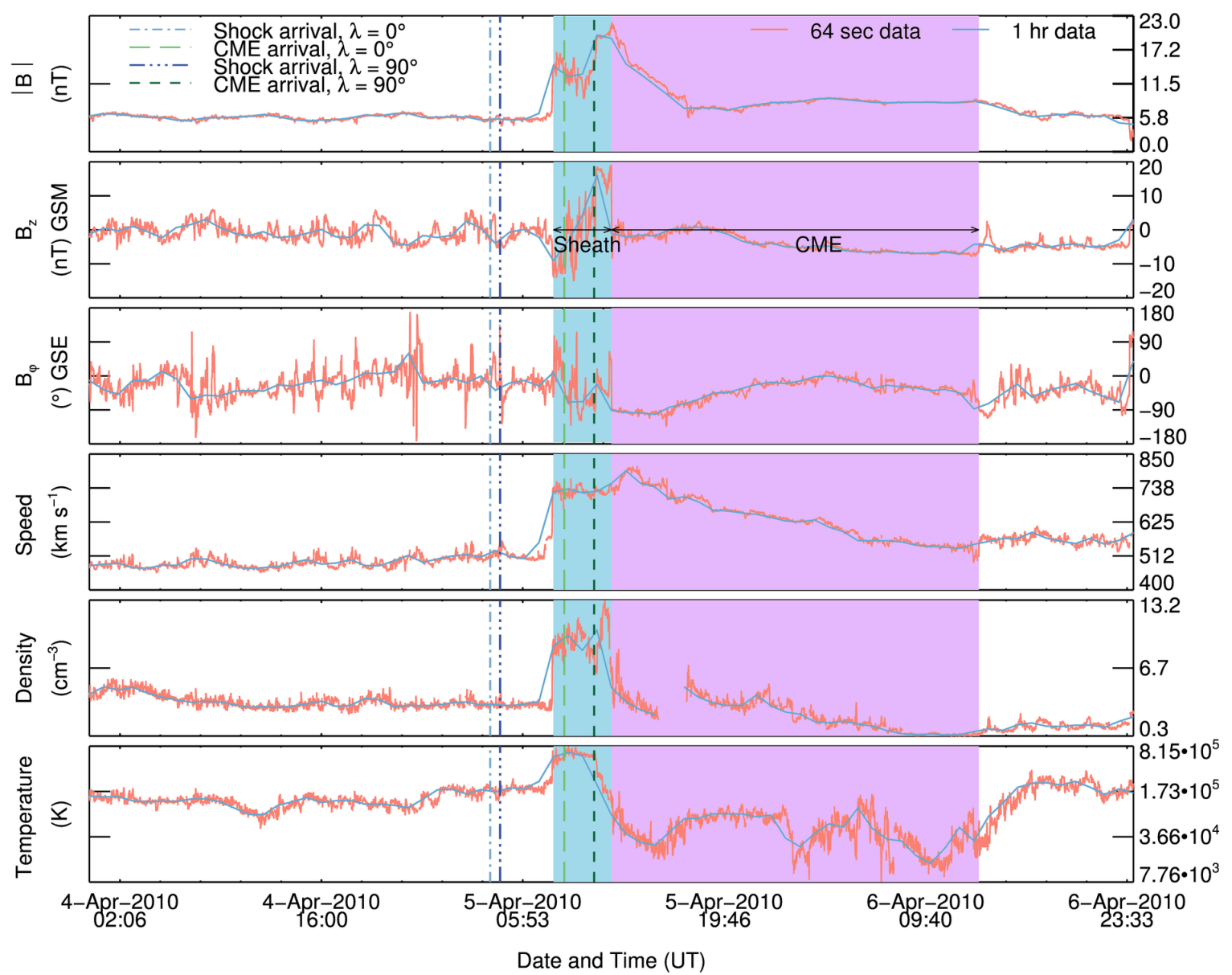

Figure 8.13.: ACE in-situ plasma and magnetic field measurements between 04 and 07 April 2010. The blue area indicates the shock sheath region, and the purple area marks the CME passage. Overplotted are the SSSEM arrival times for $\lambda=0^{\circ}$ and $\lambda=90^{\circ}$ obtained via a quadratic fit to the CME and shock SSSEM heliocentric distance. 


\begin{tabular}{llll}
\hline & EUVI+ACE & 1st degree fit $\lambda=0^{\circ}$ & 1st degree fit $\lambda=90^{\circ}$ \\
\hline CME eruption time & 03-Apr-2010 09:04 & 03-Apr-2010 19:51 & 03-Apr-2010 14:48 \\
Shock arrival time & 05-Apr-2010 07:56 & 05-Apr-2010 00:26 & 05-Apr-2010 00:19 \\
Shock arrival speed & 750 km s & 1025 $\mathrm{km} \mathrm{s}^{-1}$ & 1033 $\mathrm{km} \mathrm{s}^{-1}$ \\
CME arrival time & 05-Apr-2010 12:24 & 05-Apr-2010 05:24 & 05-Apr-2010 06:05 \\
\hline & & & \\
\hline & EUVI+ACE & 2nd degree fit $\lambda=0^{\circ}$ & 2nd degree fit $\lambda=90^{\circ}$ \\
\hline CME eruption time & 03-Apr-2010 09:04 & 03-Apr-2010 21:52 & 03-Apr-2010 14:56 \\
Shock arrival time & 05-Apr-2010 07:56 & 05-Apr-2010 03:38 & 05-Apr-2010 04:19 \\
Shock arrival speed & 750 km s & 867 $\mathrm{km} \mathrm{s}^{-1}$ & 851 km s \\
CME arrival time & 05-Apr-2010 12:24 & 05-Apr-2010 08:44 & 05-Apr-2010 10:48 \\
\hline
\end{tabular}

Table 8.5.: Observed CME eruption time, arrival time and speed at $L_{1}$ compared to those obtained via a linear (top panel) and quadratic (bottom panel) fit to the CME height-time profile derived by applying the SSSEM for $\lambda=0^{\circ}$ and $\lambda=90^{\circ}$ for the 03 April 2010 event.

blue area in the plot represents the shock sheath region, and the purple area the CME. The CME does not present the classical magnetic cloud signatures, but the declining speed profile, the low variance of the magnetic field and the low temperature are all indications of the passage of the CME across the spacecraft. Möstl et al. (2010) related the observed in-situ signatures to the passage of the CME flanks across ACE, rather than the CME apex. The negative $B_{z}$ values associated to the CME were most likely the cause of the geomagnetic storm with Kp 8- which was recorded on 05 April 2010.

A comparison between the observed and extrapolated in-situ CME and shock arrival times, shock arrival speed, and CME eruption time is presented in Table 8.5, which includes the results for $\lambda=0^{\circ}$ and $\lambda=90^{\circ}$. The top and bottom tables respectively present values obtained via a linear and a quadratic fit to CME and shock SSSEM heights. The first involves the assumption of propagation at constant speed, the second accounts for the effects of deceleration. The linear fit predicts higher arrival speeds and earlier arrival times than the ones observed by ACE. This applies to both the CME and the shock and for both values of the SSSEM half-width. The inclusion of deceleration significantly improves the arrival time estimates, especially in the case of $\lambda=90^{\circ}$, where the discrepancy between predicted and observed arrival times is as low as 2 hours. The arrival speeds are in this case are much closer to the observed values than the ones obtained for the constant velocity fit.

The linear and quadratic fits to the shock and CME height were also used to extrapolate the values of the shock standoff distance to $L_{1}$. This in turn allowed the computation of the Mach number and density compression ratio at the same location. The standoff distance and the shock parameters were compared to the observed values. The observed standoff distance $\Delta=17 R_{\odot}$ was derived directly from measurements of the $\mathrm{CME}$ and shock speed and arrival time at $\mathrm{ACE}^{8}$. The Mach number and compression ratio were compared to the values found on the Harvard Smithsonian Centre for As-

\footnotetext{
${ }^{8}$ The results presented here are slightly different from the ones presented in Volpes \& Bothmer (2015) because of improvements in the methods for the determination of the best fit to observations and for computation of the standoff distance from ACE in-situ data.
} 


\begin{tabular}{llll}
\hline & $\mathrm{ACE}$ & 1st degree fit $\lambda=10^{\circ}$ & 2nd degree fit $\lambda=10^{\circ}$ \\
\hline Standoff distance & $17 R_{\odot}$ & $30 R_{\odot}$ & $26 R_{\odot}$ \\
Compression ratio & 2.84 & 0.75 & 2.82 \\
Mach number & 2.2 & 1.38 & 3.26 \\
\hline & $\mathrm{ACE}$ & 1 st degree fit $\lambda=90^{\circ}$ & 2 nd degree fit $\lambda=90^{\circ}$ \\
\hline Standoff distance & $17 R_{\odot}$ & $29 R_{\odot}$ & $26 R_{\odot}$ \\
Compression ratio & 2.84 & 0.78 & 2.91 \\
Mach number & 2.2 & 1.41 & 3.46 \\
\hline
\end{tabular}

Table 8.6.: Comparison between standoff distance, compression ratio, and Mach number for the 03 April 2010 event as derived from ACE in-situ data, and from the linear and quadratic fit to the SSSEM heights for $\lambda=10^{\circ}$ and $\lambda=90^{\circ}$.

trophysics interplanetary shock database ${ }^{9}$. Their values are $\frac{\rho_{d}}{\rho_{u}}=2.84$ and $M=2.2$. The comparison is shown in Table 8.6 for the linear and quadratic fits to the CME and shock heights derived for the cases $\lambda=10^{\circ}$ and $\lambda=90^{\circ}$. The $\lambda=10^{\circ}$ case yields non-physical values for the compression ratio both for the first and the second degree fit. The standoff distance is overestimated in all cases, but the observed values of the shock parameters fall within the ranges determined in this analysis. For propagation at constant speed the best fit to observations is the one corresponding to $\lambda=50^{\circ}$, for which $\Delta=27 R_{\odot}, \frac{\rho_{d}}{\rho_{u}}=2.44$ and $M=2.69$. For propagation at constant deceleration the best fit to observations is the one corresponding to $\lambda=40^{\circ}$, for which $\Delta=23 R_{\odot}$, $\frac{\rho_{d}}{\rho_{u}}=2.63$ and $M=2.95$. The inclusion of deceleration not only improves the arrival time estimates for the CME and the shock, but also reduces the discrepancy in the extrapolated standoff distance.

\subsubsection{Corrections for off-Ecliptic propagation}

As discussed in Section 7.0.4, the models for the determination of the shock parameters were developed using the standoff distance and the radius of curvature computed at the stagnation line. In the case of CME-shock systems this translates to the CME apex. When applying the SSSEM, on the other hand, these values are computed along the Sun-Earth line. In order to estimate the level of inaccuracy associated to use of the SSSEM, the shock parameters were computed at the CME nose. To this purpose the

\begin{tabular}{lll}
\hline Relative error for & 1st degree fit $\lambda=50^{\circ}$ & 2nd degree fit $\lambda=40^{\circ}$ \\
\hline Standoff distance & $12 \%$ & $13 \%$ \\
CME curvature & $15 \%$ & $21 \%$ \\
Compression ratio & $3 \%$ & $6 \%$ \\
Mach number & $3 \%$ & $9 \%$ \\
\hline
\end{tabular}

Table 8.7.: Relative difference between the standoff distance, the CME radius of curvature, the compression ratio and the Mach number computed on the Ecliptic plane and at the CME apex for the 03 April 2010 event. The results for the value of lambda which yields the best fit to the in-situ shock parameters are shown.

\footnotetext{
${ }^{9}$ https://www.cfa.harvard.edu/shocks/
} 
GCS results for the latitude of the CME apex were used and a spherical geometry was assumed. The formulae used for these computations are described in Section 7.0.4, and the full treatment of the problem can be found in Volpes \& Bothmer (2015). Table 8.7 shows the relative discrepancy between values computed at the CME nose and at its apex for the cases $\lambda=40^{\circ}$ and $\lambda=50^{\circ}$. These correspond to the best fits to observations for the linear and quadratic fit to the SSSEM CME and shock heights. The results show that the shock parameters do not change substantially between the two locations. The highest discrepancy is associated to the radius of curvature for the second degree fit. The differences appear however reasonable, and the SSSEM results can be considered valid on the basis of this analysis.

The event presented in this section erupted on 03 April 2010 from NOAA AR 11059. It was associated with a B\%.4 flare, the eruption of a prominence, EUV dimmings, a EUV wave, and post-eruptive arcades.

Geometrical modelling via the GCS model shows that the CME expanded self-similarly, and that its speed decreased from an initial value of $1038 \mathrm{~km} \mathrm{~s}^{-1}$ between 12:08 UT and 17:29 UT on 03 April 2010 to $1003 \mathrm{~km} \mathrm{~s}^{-1}$ between 17:29 UT and 20:49 UT on 03 April 2010. The GCS heights compare well with the results obtained via the application of the SSSEM.

Both white-light observations and time-elongation plots show signatures of a CMEdriven shock. The kinematics of the CME and the shock could therefore be determined separately.

The CME and shock speed profiles both exhibit an initial increase and a subsequent deceleration. Propagation at heliocentric distances larger than $\simeq 30 R_{\odot}$ happens roughly at constant speed. The average direction of propagation for the CME and the shock is compatible with radial propagation along the Sun-Earth line. In general very similar results are obtained for all the values of the SSSEM half-width. A comparison between $A C E$ in-situ measurements and arrival time and speed extrapolations at $L_{1}$ shows that the best results are obtained for $\lambda=90^{\circ}$ and propagation at constant deceleration. The corresponding CME and shock arrival time estimates are only respectively $\simeq 2$ and $\simeq 3.5$ hours earlier than the ACE arrival time. The predicted arrival speed, $851 \mathrm{~km} \mathrm{~s}^{-1}$, is in good agreement with the observed value of $750 \mathrm{~km} \mathrm{~s}^{-1}$.

The temporal evolution of the shock standoff distance, compression ratio, and Mach number was derived based on the results for the CME and shock kinematics. Their values were extrapolated to $L_{1}$ using the linear and quadratic fit to the CME and shock heliocentric distances. The comparison with ACE in-situ data shows that the best fit to observations is associated to $\lambda=50^{\circ}$ when propagation at constant speed is assumed, and to $\lambda=40^{\circ}$ for propagation at constant deceleration. The former yields $\Delta=27 R_{\odot}$, $\frac{\rho_{d}}{\rho_{u}}=2.44$ and $M=2.69$, the latter yields $\Delta=23 R_{\odot}, \frac{\rho_{d}}{\rho_{u}}=2.63$ and $M=2.95$. The 
values of the shock parameters derived from ACE in-situ data, on the other hand, are $\Delta=17 R_{\odot}, \frac{\rho_{d}}{\rho_{u}}=2.84$ and $M=2.2$.

The calculation of the fitting errors shows that accounting for the effects of deceleration in the CME and shock propagation yields more accurate predictions not only for the arrival times and speeds, but also for the shock parameters at $L_{1}$ 


\subsection{The 07 June $2011 \mathrm{CME}$}

\subsubsection{Solar source region}

The 07 June 2011 CME originated from NOAA AR 11226, located on the south west quadrant of the solar disk (S22W53). It was associated to a massive prominence eruption, shown in the EUV images of Figure $8.14^{10}$. SDO/AIA observations show a flare, a dimming and southward propagating wave associated with the event. An M2.5 flare was also recorded by GOES beginning at 06:16 UT. The STEREO A and B spacecraft
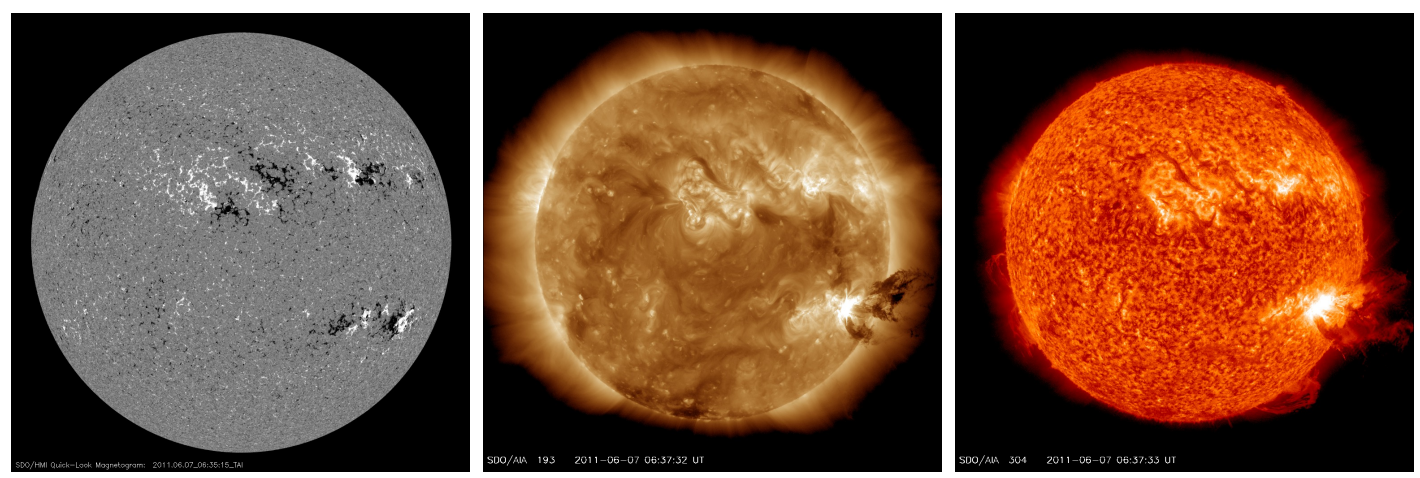

Figure 8.14.: SDO MDI, AIA $193 \AA$ and AIA $304 \AA$ observations of the solar disk on 07 June 2011. Images courtesy of the NASA SDO consortium.

observed the CME during its interplanetary propagation. The angular separations between each STEREO A and B and Earth on 07 June 2011 were respectively $94^{\circ}$ and $93^{\circ}$. Consequently the source region appears to lie on the SE quadrant in STEREO EUVI A images and is backsided from the STEREO B perspective. Subsequent phases of the CME interplanetary propagation are shown in Figure 8.15. COR2 A first observed the event on 07 June 2011 at 06:54 UT, and COR2 B first observed the event on 07 June 2011 at 07:09 UT. The CME crossed the HI1 A field of view on 07 June 2011 at 08:49 UT, and the HI1 B field of view on 07 June 2011 at 09:29 UT. HI2 A and B first observed the CME respectively on 07 June 2011 at 20:09 UT and 08 June 2011 on 02:09 UT. Shock signatures are visible in COR2 A and B images, and even in HI1 B. The complex morphology of the event, though, makes it difficult to distinguish between the CME front and the shock in HI1 A observations. An investigation of the CME and shock kinematics was attempted for this event by using single spacecraft fitting techniques. A modified SSEF was used, in which $\lambda$ was treated as an input parameter. The results are shown below.

\subsubsection{GCS modelling}

The CME geometry was fitted via the GCS model. The COR2 modelling parameters available on the AFFECTS database ${ }^{11}$ were used. The corresponding GCS flux rope

\footnotetext{
${ }^{10}$ NASA issued a press-release for the 07 June 2011 CME on https://www.nasa.gov/mission pages/sunearth/news/News060711-blast.html

${ }^{11}$ http://www.affects-fp7.eu/cme-database/database.php
} 

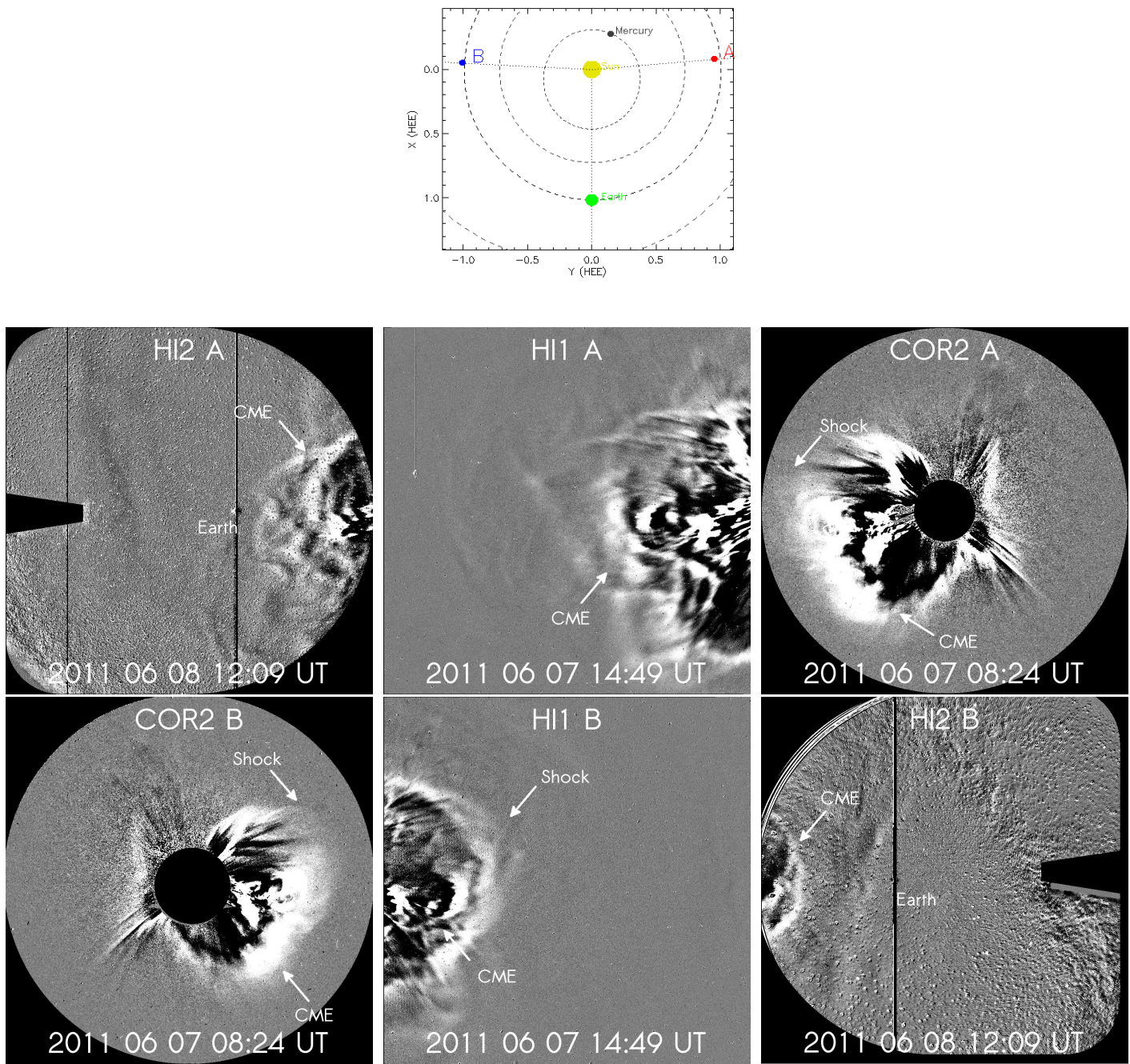

Figure 8.15.: Top panel: positions of the STEREO spacecraft on 07 June 2011. Image credit: NASA STEREO consortium. Bottom panel: difference images obtained from STERE$\mathrm{O} / \mathrm{SECCHI} \mathrm{A}$ and B COR2 HI1 and HI2 observations, showing subsequent phases of the CME evolution for the 07 June 2011 event.

was extended to the HI1 field of view by keeping all the model parameters fixed except for the CME height. The extension to the HI1 field of view, however, was not straightforward due to the complex CME morphology. Figure 8.16 shows coronagraph and heliospheric imager observations of the CME with the superimposed GCS model. The derived GCS parameters are shown in Table 8.8. The average CME apex speed decreased from $1136 \mathrm{~km} \mathrm{~s}^{-1}$ between 08:15 and 12:49 UT to $776 \mathrm{~km} \mathrm{~s}^{-1}$ between 12:49 and 14:49 UT. The corresponding average acceleration was $-15.21 \mathrm{~m} \mathrm{~s}^{-2}$ Such values, however, should be interpreted carefully, as the application of the GCS model to HI1 observations for this particular event is quite challenging due to the complex CME morphology. The values of the GCS apex heights do not seem to be in good agreement with those derived via the application of the SSEF. The large errors in the SSEF heights, though, make it hard to state the compatibility of the GCS and SSEF results. 
The latter yield $44 \pm 43 R_{\odot}$ on 07 June 2011 at $12: 49$ and $70 \pm 50 R_{\odot}$ on 07 June 2011 at 14:49 for $\lambda=0^{\circ}$ and $93 \pm 83 R_{\odot}$ on 07 June 2011 at $12: 49$ and $115 \pm 88 R_{\odot}$ on 07 June 2011 at $14: 49$ for $\lambda=90^{\circ}$.
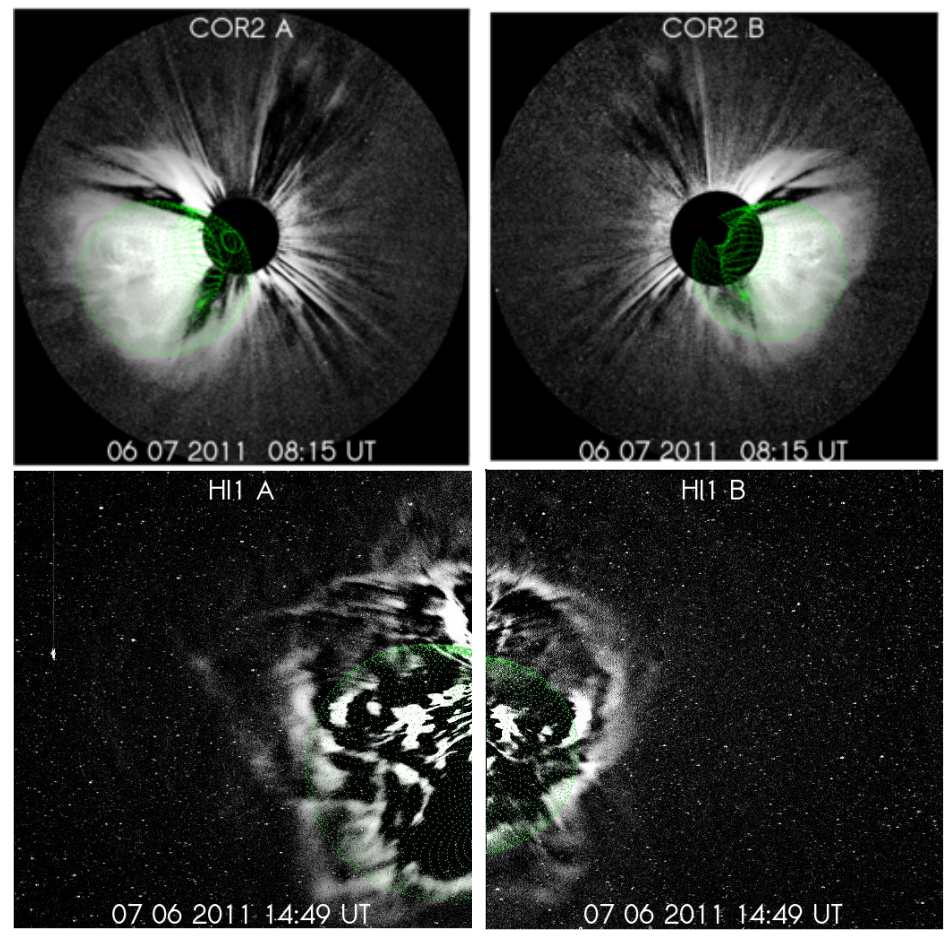

Figure 8.16.: GCS modelling for STEREO/SECCHI COR2 (upper panel) and HI1 (lower panels) observations of the 07 June $2011 \mathrm{CME}$ at 08:15 UT and 14:29 UT.

\begin{tabular}{|r|l|l|l|l|l|l|l|r|}
\hline Instrument & Time & Lon & Lat & Tilt & Height & $\mathrm{k}$ & $\alpha$ & $\mathrm{a}$ \\
\hline COR2 & 2011-Jun-07 08:15:00 & $33.54^{\circ}$ & $-13.97^{\circ}$ & $-5.03^{\circ}$ & $13.07 R_{\odot}$ & 0.6 & 10.34 & $7.84 R_{\odot}$ \\
HI1 & 2011-Jun-07 12:49:00 & $33.54^{\circ}$ & $-13.97^{\circ}$ & $-5.03^{\circ}$ & $39.93 R_{\odot}$ & 0.6 & 10.34 & $23.96 R_{\odot}$ \\
HI1 & 2011-Jun-07 14:49:00 & $33.54^{\circ}$ & $-13.97^{\circ}$ & $-5.03^{\circ}$ & $47.97 R_{\odot}$ & 0.6 & 10.34 & $28.78 R_{\odot}$ \\
\hline
\end{tabular}

Table 8.8.: GCS modelling results for the 07 June 2011 CME. The table shows the imaging instrument, the time of observations, and the GCS parameters: Carrington longitude, heliographic latitude and tilt angle of the solar source region, flux rope height $R_{G C S}$, aspect ratio $k$, half-angle $\alpha$, and radial width $a=k R_{G C S}$.

\subsubsection{SSEF results}

Figure 8.17 shows the j-map obtained from cuts along the Ecliptic plane of STEREO B observations between 06 and 11 June 2011. The track originating on 07 June 2011 corresponds to the event analysed in this Section. A similar plot for STEREO A was obtained, but is not shown, since it is not part of the analysis discussed here. The shock could in fact only be resolved in STEREO B time-elongation plots, and an attempt 
at the determination of the $\mathrm{CME}$ and shock kinematics via the implementation of the SSEF was performed. As mentioned above, the SSEF method was slightly modified and $\lambda$ was used as an input parameter rather than considered as one of the fitting results. The fit to the time-elongation measurements was performed in the range $10^{\circ}<\lambda<90^{\circ}$,

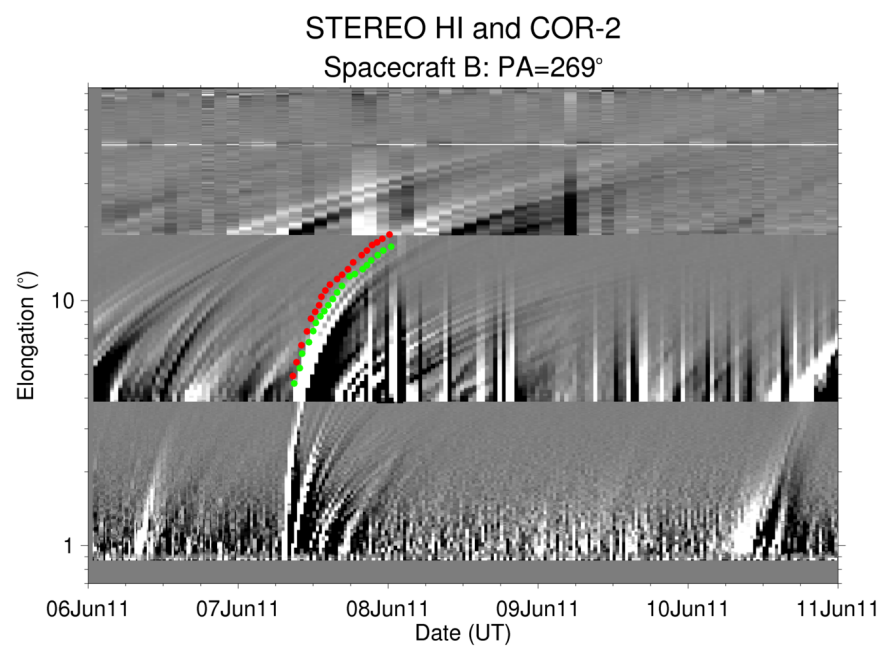

Figure 8.17.: Top panel: j-maps obtained from STEREO B observations between 06 and 11 June 2011. The red dots represent the time-elongation measurements for the shock, and the green dots are the time-elongation pairs measured for the CME.

with $\lambda$ varying in steps of $10^{\circ}$. It should be remarked that the fitting technique used to analyse this event assumes that the tracked solar wind feature propagates at constant speed and at constant direction with respect to the Sun-Earth line. These parameters are the results of the fit. The time-elongation measurements for the CME and the shock are illustrated in Figure 8.17. The red dots correspond to measurements taken for the shock, whereas the green dots are relative to the CME leading edge.

\section{CME}

Figure 8.18 shows the temporal evolution of the CME height obtained by applying the SSEF to time-elongation measurements performed on $\mathrm{j}$-maps derived from STEREO B observations. Results are shown for $\lambda=0^{\circ}$ and $\lambda=90^{\circ}$. The fitting methods assume that the tracked solar wind feature propagates at constant speed $v$ and at and constant angle of propagation on the Ecliptic plane $\delta_{E}$. Such parameters are a result of the fit itself, and are illustrated in Table 8.9. They are $v=1276 \mathrm{~km} \mathrm{~s}^{-1}$ and $\delta_{E}=36^{\circ}$ for $\lambda=0^{\circ}$ and $v=2306 \mathrm{~km} \mathrm{~s}^{-1}$ and $\delta_{E}=92^{\circ}$ for $\lambda=90^{\circ}$. Based on the direction of propagation the CME did not impact Earth ${ }^{12}$. The method also yields eruption times, namely 07 June 2011 at 04:49 UT for $\lambda=0^{\circ}$ and 07 June 2011 04:58 UT for $\lambda=90^{\circ}$. These compare well with the CME onset time as derived from EUV observations.

\footnotetext{
${ }^{12}$ the SSEF results are compatible with an impact on Earth only if $\delta_{E}<\lambda$.
} 


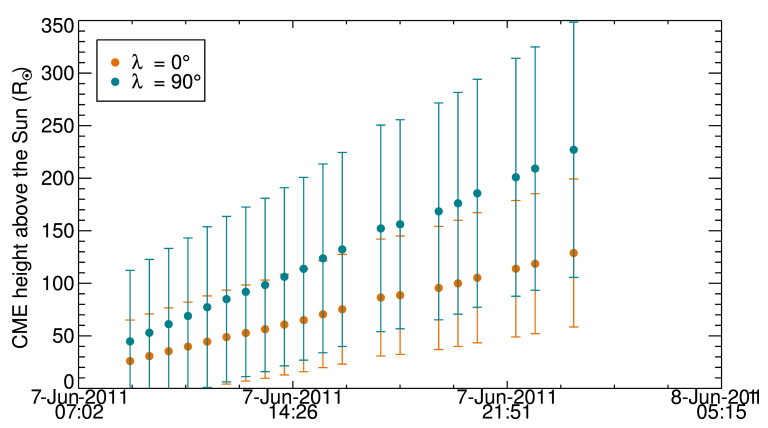

\begin{tabular}{|c|c|c|}
\hline & $\lambda=0^{\circ}$ & $\lambda=90^{\circ}$ \\
\hline $\mathrm{v}$ & $1276 \mathrm{~km} \mathrm{~s}^{-1}$ & $2306 \mathrm{~km} \mathrm{~s}^{-1}$ \\
\hline$\delta_{E}$ & $36^{\circ}$ & $92^{\circ}$ \\
\hline
\end{tabular}

Figure 8.18.: Time evolution of the CME height above the Sun obtained via the application of the SSEF technique for $\lambda=0^{\circ}$ and $\lambda=90^{\circ}$ to STEREO B observations of the 07 June 2011 event.

Table 8.9.: CME speed and direction of propagation with respect to the Sun-Earth line obtained via the application of the SSEF technique for $\lambda=0^{\circ}$ and $\lambda=90^{\circ}$ STEREO B observations of the 07 June 2011 event.

\section{Shock}

The results obtained by the application of the SSEF to time-elongation measurements obtained for the CME-driven shock are shown in Figure 8.19, which illustrates the temporal evolution of the shock height. The SSEF fits results are included in Table 8.10. The shock speeds and direction of propagation are $v=1657 \mathrm{~km} \mathrm{~s}^{-1}$ and $\delta_{E}=39^{\circ}$ for $\lambda=0^{\circ}$ and $v=3387 \mathrm{~km} \mathrm{~s}^{-1}$ and $\delta_{E}=99^{\circ}$ for $\lambda=90^{\circ}$. The derived shock speeds are higher than those obtained for the CME for both values of $\lambda$. This is in agreement with the observations, where the shock is seen to progressively move away from the CME. Similarly to what found for the CME, the shock direction of propagation is not compatible with an impact on Earth.

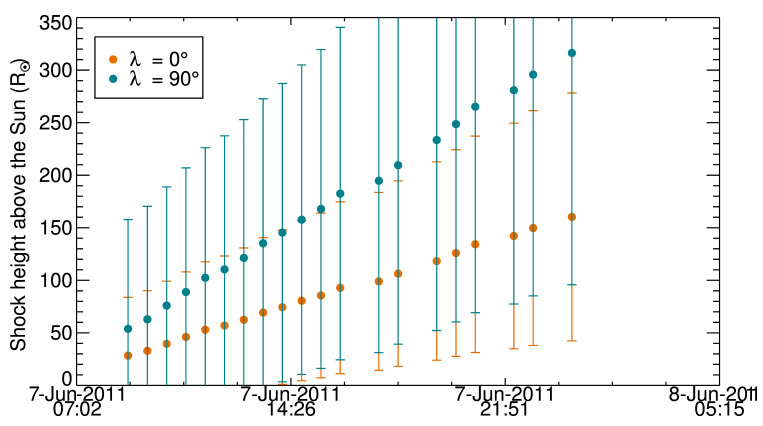

Figure 8.19.: Time evolution of the shock height above the Sun obtained via the application of the SSEF technique for $\lambda=0^{\circ}$ and $\lambda=90^{\circ}$ to STEREO B observations of the 07 June 2011 event.

\begin{tabular}{|c|c|c|}
\hline & $\lambda=0^{\circ}$ & $\lambda=90^{\circ}$ \\
\hline $\mathrm{v}$ & $1657 \mathrm{~km} \mathrm{~s}^{-1}$ & $3387 \mathrm{~km} \mathrm{~s}^{-1}$ \\
\hline$\delta_{E}$ & $39^{\circ}$ & $99^{\circ}$ \\
\hline
\end{tabular}

Table 8.10.: Shock speed and direction of propagation with respect to the Sun-Earth line obtained via the application of the SSEF technique of the 07 June 2011 event for $\lambda=0^{\circ}$ and $\lambda=90^{\circ}$ to STEREO B observations of the 07 June 2011 event. 


\subsubsection{CME-driven shock parameters}

Figure 8.20 shows the temporal evolution of the shock parameters obtained from the application of single spacecraft fitting models (SSEF) to STEREO B observations in combination with models for the shock location.
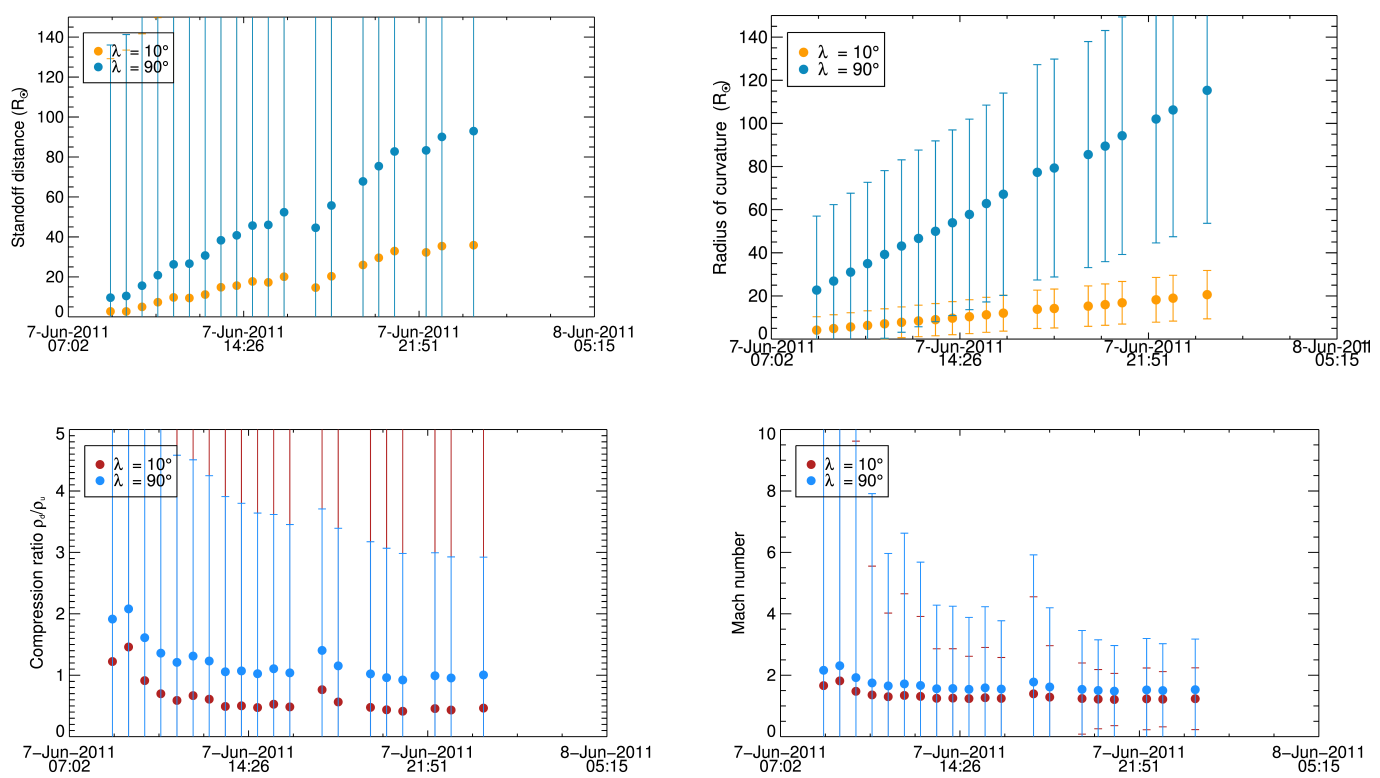

Figure 8.20.: Time evolution of the CME radius of curvature, the shock standoff distance, the compression ratio and the Mach number obtained applying the SSEF for $\lambda=10^{\circ}$ (red and orange dots) and $\lambda=90^{\circ}$ (blue dots) to STEREO-B observations of the 07 June 2011 event.

The standoff distance and the radius of curvature of the CME increase roughly linearly with time and show a rather regular profile. The compression ratio for $\lambda=10^{\circ}$ is non-physically below 1 except for a few points in the earlier phase of propagation. Its temporal evolution shows an initial increase, followed by a decrease, and finally a constant behaviour. The Mach number shows a similar time profile, although with less variation. Unfortunately, all the shock parameters are affected by very large errors which hinder the reliability of the results obtained for this event.

\subsubsection{In-situ comparison}

Figure 8.21 shows ACE in-situ measurements of the solar wind plasma and magnetic field parameters between 07 and 10 July 2011. No signatures of a CME passage are identifiable in the data. This confirms the prediction of the SSEF technique. Due to the lack of in-situ data, a validation of the results for both the CME kinematics and the shock parameters is not possible for this event. 


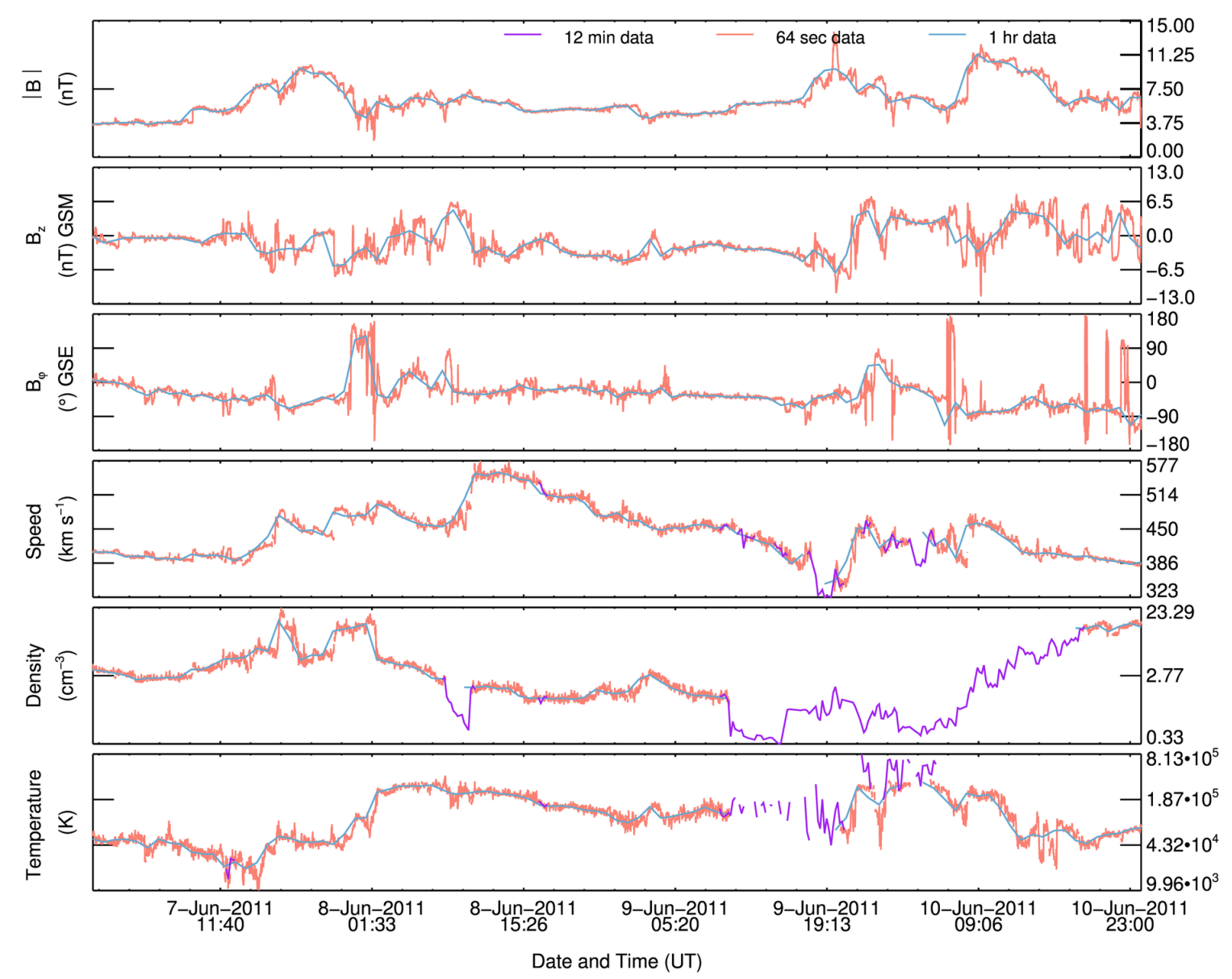

Figure 8.21.: ACE in-situ plasma and magnetic field measurements between 07 and 10 June 2011. No signatures of the passage of a CME are present in the data.

The 07 June 2011 events erupted from AR 11226. It was associated with the eruption of a large prominence, an M2.5 flare, EUV dimmings and a EUV wave.

Geometrical modelling via the GCS model shows that the apex speed decreased from $1136 \mathrm{~km} \mathrm{~s}^{-1}$ between 08:15 UT and 12:49 UT on 07 June 2011 to $776 \mathrm{~km} \mathrm{~s}^{-1}$ between 12:49 UT and 14:49 UT on the same day. Geometrical modelling for this event is however rather challenging, because of the complex CME morphology.

Signatures of a CME-driven shock are visible in STEREO B difference images and $j$ maps. The identification of the shock in STEREO A observation is more ambiguous, especially in HI1 A images. For this reason a single spacecraft based technique, namely the SSEF, was employed for the determination of the CME and shock kinematics as well as of the shock parameters. Such method assumes that the tracked solar wind feature propagates radially and at constant speed. The speed and direction of propagation for the CME were found to be $1276 \mathrm{~km} \mathrm{~s}^{-1}$ and $36^{\circ}$ for $\lambda=0^{\circ}$ and $2306 \mathrm{~km} \mathrm{~s}^{-1}$ and $92^{\circ}$ for $\lambda=90^{\circ}$. The corresponding values for the shock are $1657 \mathrm{~km} \mathrm{~s}^{-1}$ and $39^{\circ}$ for $\lambda=0^{\circ}$ and $3387 \mathrm{~km} \mathrm{~s}^{-1}$ and $99^{\circ}$ for $\lambda=0^{\circ}$. The values of the direction of propagation are not 
compatible with an impact on Earth for both the CME and the shock and all the values of $\lambda$. These results are consistent with ACE in-situ data, which show no signatures of the passage of a CME or an interplanetary shock.

The shock parameters were determined using the SSEF results in combination with models for the shock location. The standoff distance was found to increase the time, consistently with the results for the CME and shock speed. The Mach number and compression ratio show a constant profile in the later stages of the CME propagation. A slight increase followed by a decrease is seen for both parameters for CME heights lower than $80 R_{\odot}$. The case $\lambda=10^{\circ}$ yields non-physical values of the density ratio. All the parameters suffer from very large errors which are most likely a consequence of the employment of single spacecraft based techniques.

Unfortunately the results presented for the 07 June 2011 could not be verified against in-situ plasma and magnetic field measurements since neither the CME nor the shock were detected by $A C E$. 


\subsection{The 03 August $2011 \mathrm{CME}$}

\subsubsection{Solar source region}

The 03 August 2011 CME erupted at about 13:15 UT from NOAA AR 11261. The active region location at the date of CME eruption was N16W28. An M6.0 flare was recorded by GOES between 13:17 and 14:21 UT. EUV dimmings and a wave were associated with this event and post eruptive arcades can be seen in SDO EUV images. Figure 8.22 shows SDO EUV observations of the solar disk around the time of CME
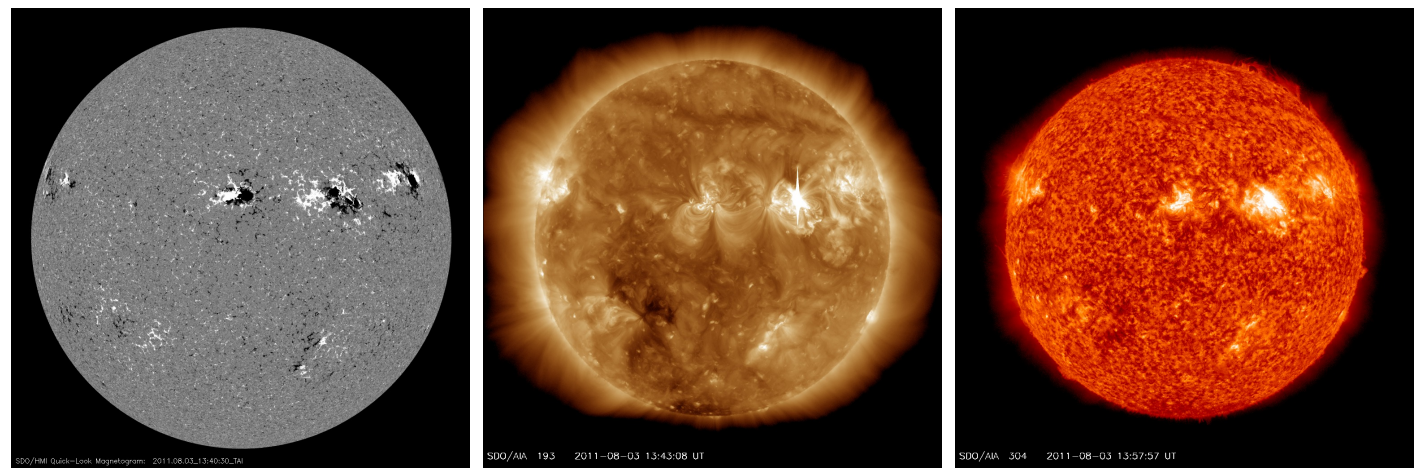

Figure 8.22.: SDO HMI, AIA $193 \AA$ and $304 \AA$ observations of the solar disk on 03 August 2011. Images courtesy of the NASA SDO consortium.

eruption at $193 \AA$ and $304 \AA$ as well as an HMI magnetogram showing the magnetic polarity of the CME source region. The separation angles between STEREO A and B and Earth at that time were $100^{\circ}$ and $92^{\circ}$ respectively. The CME therefore appeared as erupting from the NE quadrant from STEREO A, and from the NW quadrant from STEREO B. The event was first observed in COR2 at 13:54 UT. It entered the HI1 field of view at 15:29 UT, and the HI2 field of view at 02:09 UT on 04 August 2011. Figure 8.23 shows subsequent phases of the CME propagation. A bright forerunner is seen in coronagraph and heliospheric imager observations, corresponding to signatures of a CME-driven shock. A separate analysis of CME and shock kinematics, as well as the derivation of the temporal evolution of the shock parameters, was possible for this event.

\subsubsection{GCS modelling}

The CME front was fitted to the GCS geometry by using the COR2 modelling parameters available through the AFFECTS database. The corresponding geometry was extended to the HI1 field of view by keeping all the parameters constant except for the CME height. The results are presented in Table 8.11. Figure 8.24 shows the GCS flux rope superimposed on STEREO A and B observations. The GCS model performs fairly well in reproducing the observed geometry, except for the elongated white light feature at the CME apex. This feature becomes increasingly large as the CME expands outwards, and consequently the GCS model is less and less accurate in describing the CME geometry for this event. The average apex velocity between 15:08 and 18:49 UT 

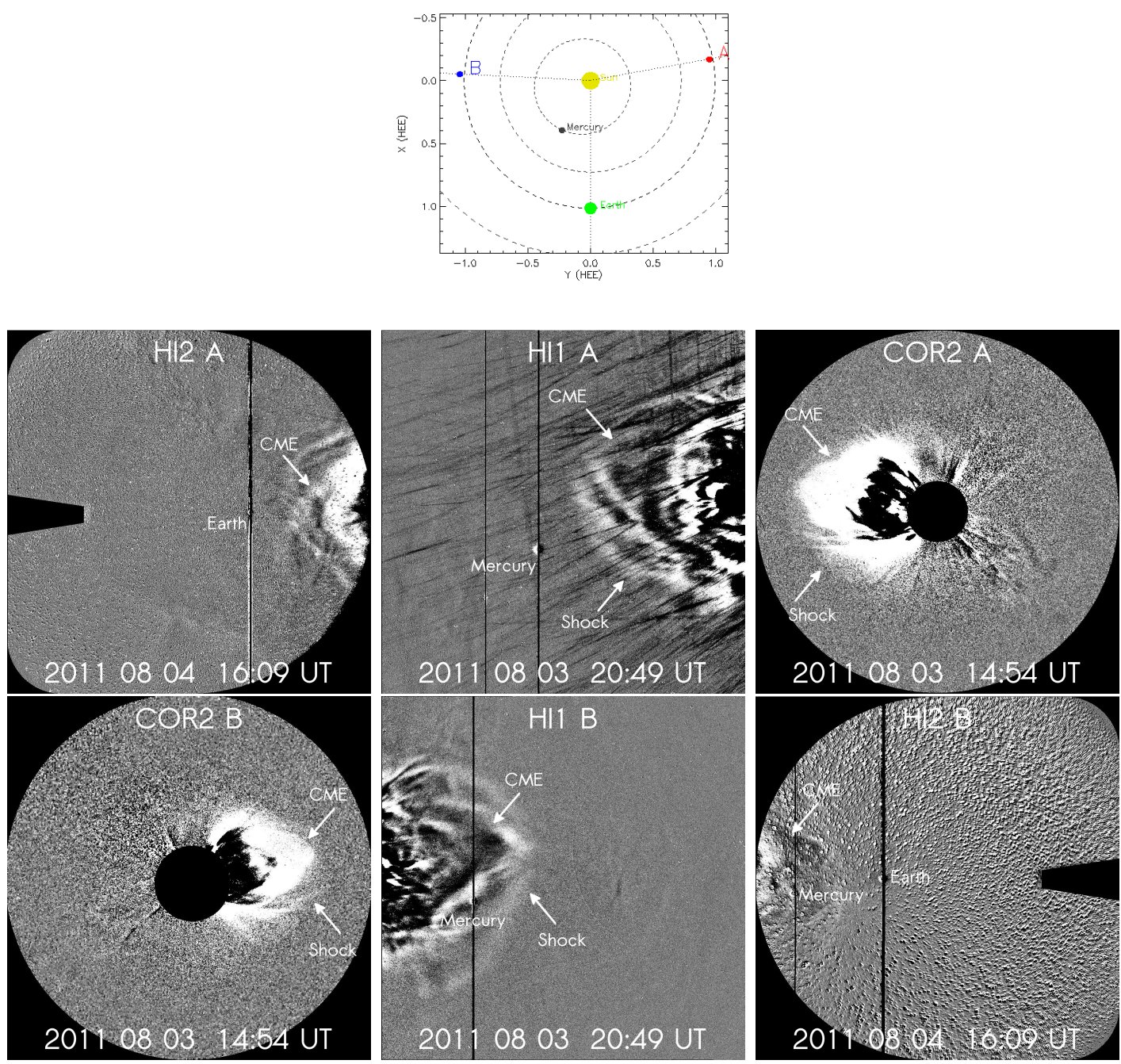

Figure 8.23.: Top panel: positions of the STEREO spacecraft on 03 August 2011. Image credit: NASA STEREO consortium. Bottom panel: difference images obtained from STERE$\mathrm{O} /$ SECCHI A and B COR2 HI1 and HI2 observations, showing subsequent phases of the CME evolution for the 03 August 2011 event.

was $1143 \mathrm{~km} \mathrm{~s}^{-1}$, and it decreased to a value of $818 \mathrm{~km} \mathrm{~s}^{-1}$ between 18:49 and 23:29 UT. The acceleration had an average a value of $-10.80 \mathrm{~m} \mathrm{~s}^{-2}$ The CME radial width increased from $6.53 R_{\odot}$ at 15:08 UT to $28.15 R_{\odot}$ at 23:29 UT. A comparison to the SSSEM heights obtained for the CME shows a very good agreement with the GCS results. For $\lambda=0^{\circ}$ we find $33 \pm 1 R_{\odot}$ on 03 August 2011 at 18:49 UT, and $56 \pm 1 R_{\odot}$ at 23:49 UT. For $\lambda=90^{\circ}$, similarly, we find $31 \pm 1 R_{\odot}$ on 03 August 2011 at 18:49 $\mathrm{UT}$, and $54 \pm 8 R_{\odot}$ at 23:49 UT, values very close to the ones obtained via the GCS fit. Since the SSSEM was employed only for time-elongation measurements in the HI1 field of view, no comparison was possible for the GCS height in COR2. 

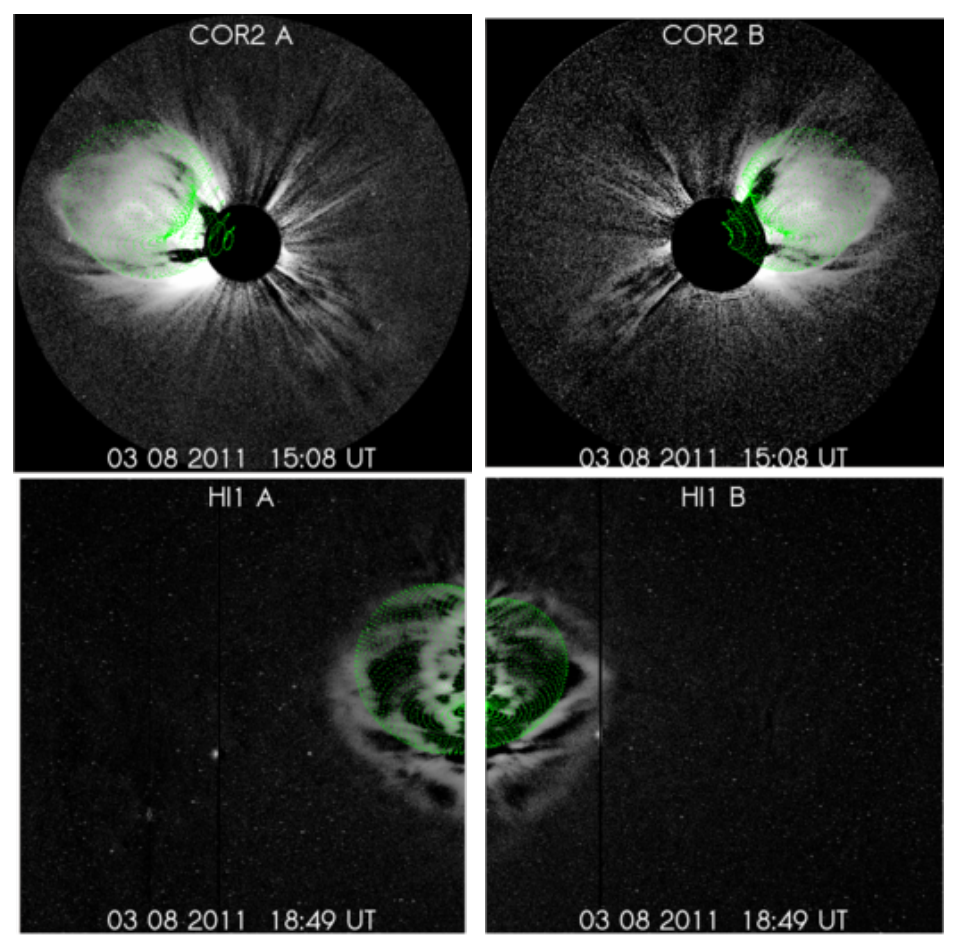

Figure 8.24.: GCS modelling for STEREO/SECCHI COR2 (upper panel) and HI1 (lower panels) observations of the 03 August $2011 \mathrm{CME}$ at 15:08 UT and 18:49 UT.

\begin{tabular}{|r|l|l|l|l|l|l|l|l|}
\hline Instrument & Time & Lon & Lat & Tilt & Height & k & $\alpha$ & a \\
\hline COR2 & 2011-Aug-03 15:08:00 & $324.22^{\circ}$ & $24.037^{\circ}$ & $-40.25^{\circ}$ & $12.57 R_{\odot}$ & 0.52 & 19.84 & $6.53 R_{\odot}$ \\
HI1 & 2011-Aug-03 18:49:00 & $324.22^{\circ}$ & $24.037^{\circ}$ & $-40.25^{\circ}$ & $34.37 R_{\odot}$ & 0.52 & 19.84 & $17.87 R_{\odot}$ \\
HI1 & 2011-Aug-03 23:29:00 & $324.22^{\circ}$ & $24.037^{\circ}$ & $-40.25^{\circ}$ & $54.15 R_{\odot}$ & 0.52 & 19.84 & $28.15 R_{\odot}$ \\
\hline
\end{tabular}

Table 8.11.: GCS modelling results for the 03 August 2011 CME. The table shows the imaging instrument, the time of observations, and the GCS parameters: Carrington longitude, heliographic latitude and tilt angle of the solar source region, flux rope height $R_{G C S}$, aspect ratio $k$, half-angle $\alpha$, and radial width $a=k R_{G C S}$.

\subsubsection{SSSEM results}

Figure 8.25 shows time-elongation plots obtained from STEREO A and B observations between 03 and 08 August 2011. They show the propagation of solar wind transients along the Ecliptic plane. The track corresponding to the CME analysed in this section appears on 03 August 2011. Since the CME and the shock could be separately identified in difference images and in time-elongation plots their evolution could be followed independently. The green dots in the j-maps corresponds to time-elongation measurements for the $\mathrm{CME}$, whereas the red dots correspond to time-elongation pairs measured at the shock front. The SSSEM was used to obtain the temporal evolution of the speed, height above the Sun, and direction of propagation for the CME and the shock. 

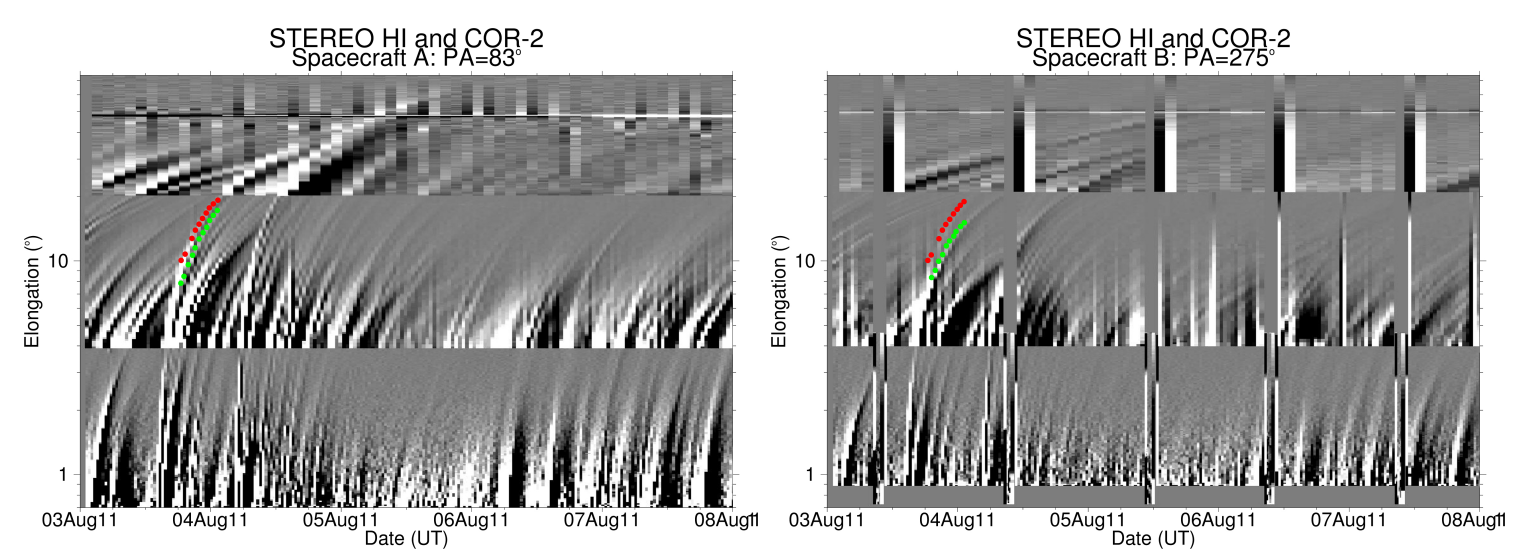

Figure 8.25.: Top panel: j-maps obtained from STEREO A (left) and B (right) observations between 04 and 08 August 2011. The red dots represent the time-elongation measurements for the shock, and the green dots are the time-elongation pairs measured for the CME.

\section{CME}

The SSSEM results obtained for $\lambda=0^{\circ}$ and $\lambda=90^{\circ}$ are shown in Figure 8.26. They show the temporal evolution of the CME height, speed, and direction of propagation with respect to the Sun-Earth line. The CME speed profile shows a roughly constant behaviour. The direction of propagation is constant, with average values $\delta_{E}=-4^{\circ} \pm 6^{\circ}$ for $\lambda=0^{\circ}$ and $\delta_{E}=-4 \pm 7^{\circ}$ for $\lambda=90^{\circ}$ compatible with radial propagation along the Sun-Earth line.
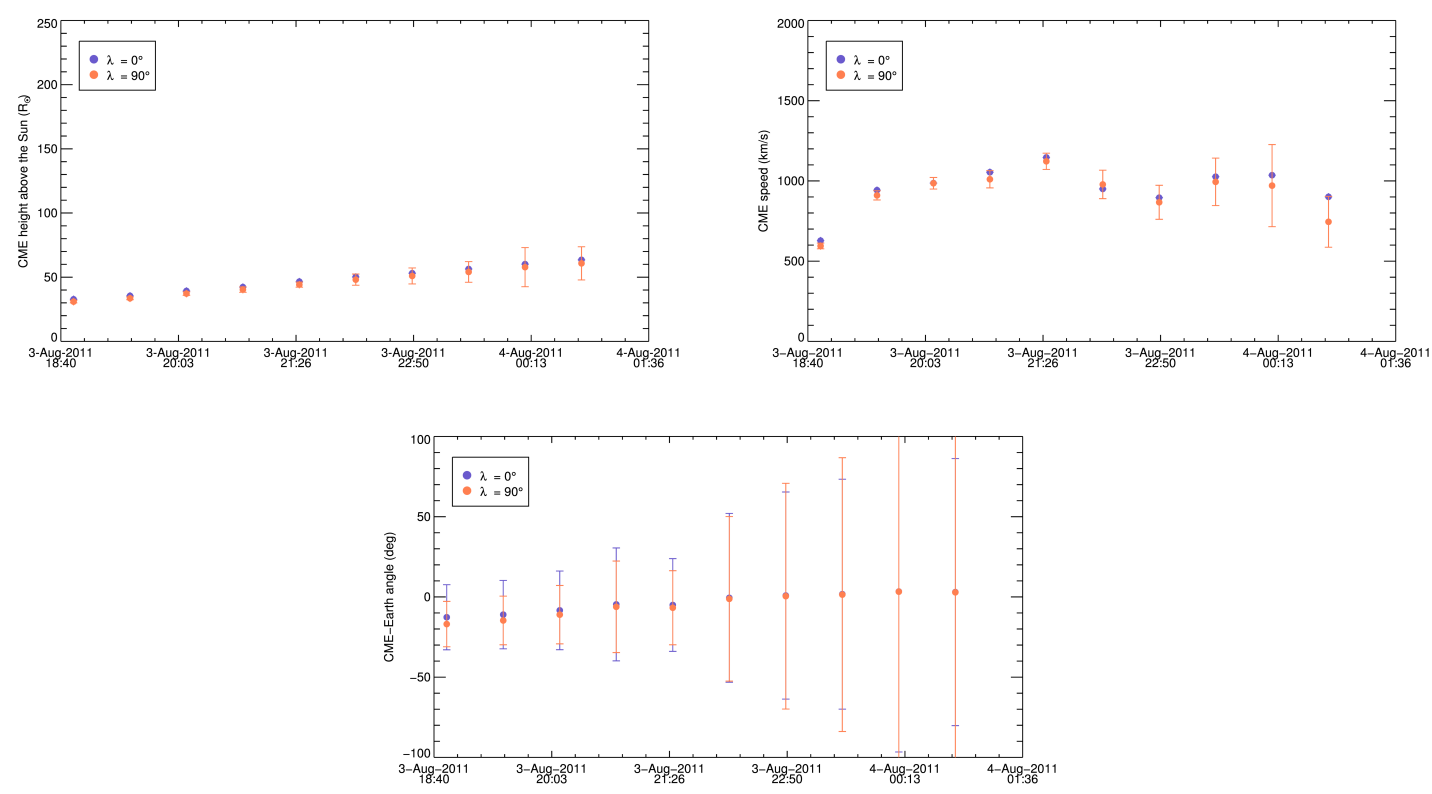

Figure 8.26.: Time evolution of the CME height, speed and direction of propagation w.r.t. the Sun-Earth line obtained by applying the SSSEM for $\lambda=0^{\circ}$ and $\lambda=90^{\circ}$ to STEREO/SECCHI observations of the 03 August 2011 event. 


\section{Shock}

The results for the shock kinematics, derived by applying the SSSEM to time-elongation pairs obtained from the j-maps, are shown in Figure 8.27 for $\lambda=0^{\circ}$ and $\lambda=90^{\circ}$. The
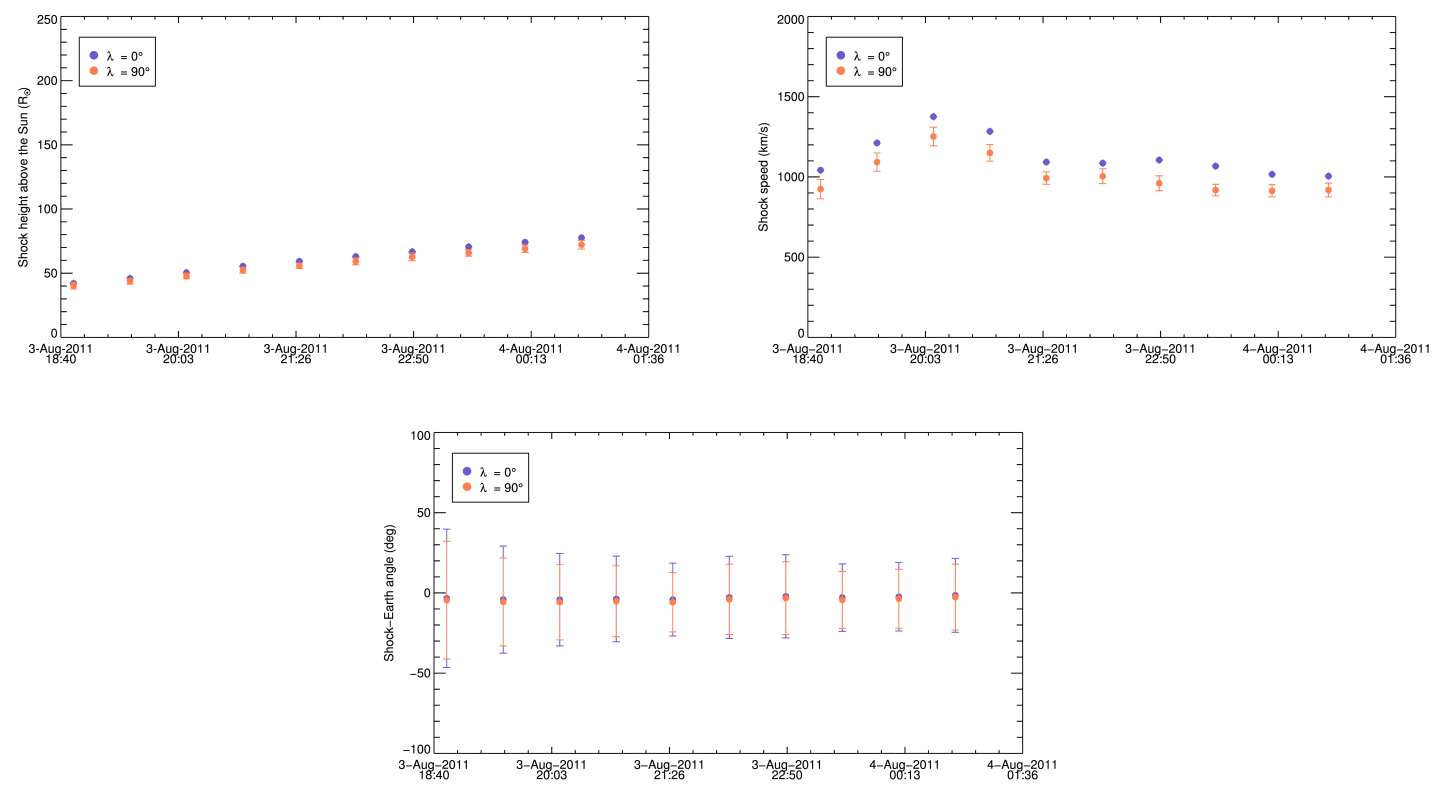

Figure 8.27.: Time evolution of the shock height, speed and direction of propagation towards Earth obtained by applying the SSSEM for $\lambda=0^{\circ}$ and $\lambda=90^{\circ}$ for the 03 August 2011 event.

shock speeds shows an initial increase and then a decrease towards a more constant profile. The direction of propagation, with average values $\delta_{E}=-4^{\circ} \pm 1^{\circ}$ for both $\lambda=0^{\circ}$ and $\lambda=90^{\circ}$, indicates radial propagation along the Sun-Earth line.

\subsubsection{CME-driven shock parameters}

The combination of the SSSEM results and the models described in Chapter 7 yields the temporal evolution of the standoff distance, the CME radius of curvature, the compression ratio and the Mach number. The results are shown in Figure 8.28. The standoff distance increases with time, although the initial and final values do not differ by a large amount. The radius of curvature for $\lambda=90^{\circ}$ is unsurprisingly larger than the one for $\lambda=10^{\circ}$. The case $\lambda=0^{\circ}$ is excluded from these results for the reasons explained in Section 7.0.4. The Mach number and the compression ratio both show a roughly constant profile, a behaviour found for other events in this and other studies (Maloney \& Gallagher, 2011). The compression ratio for $\lambda=10^{\circ}$ is smaller than one. Values lower than 1, as noted earlier in this work, are not consistent with the picture of a compressive shock. 

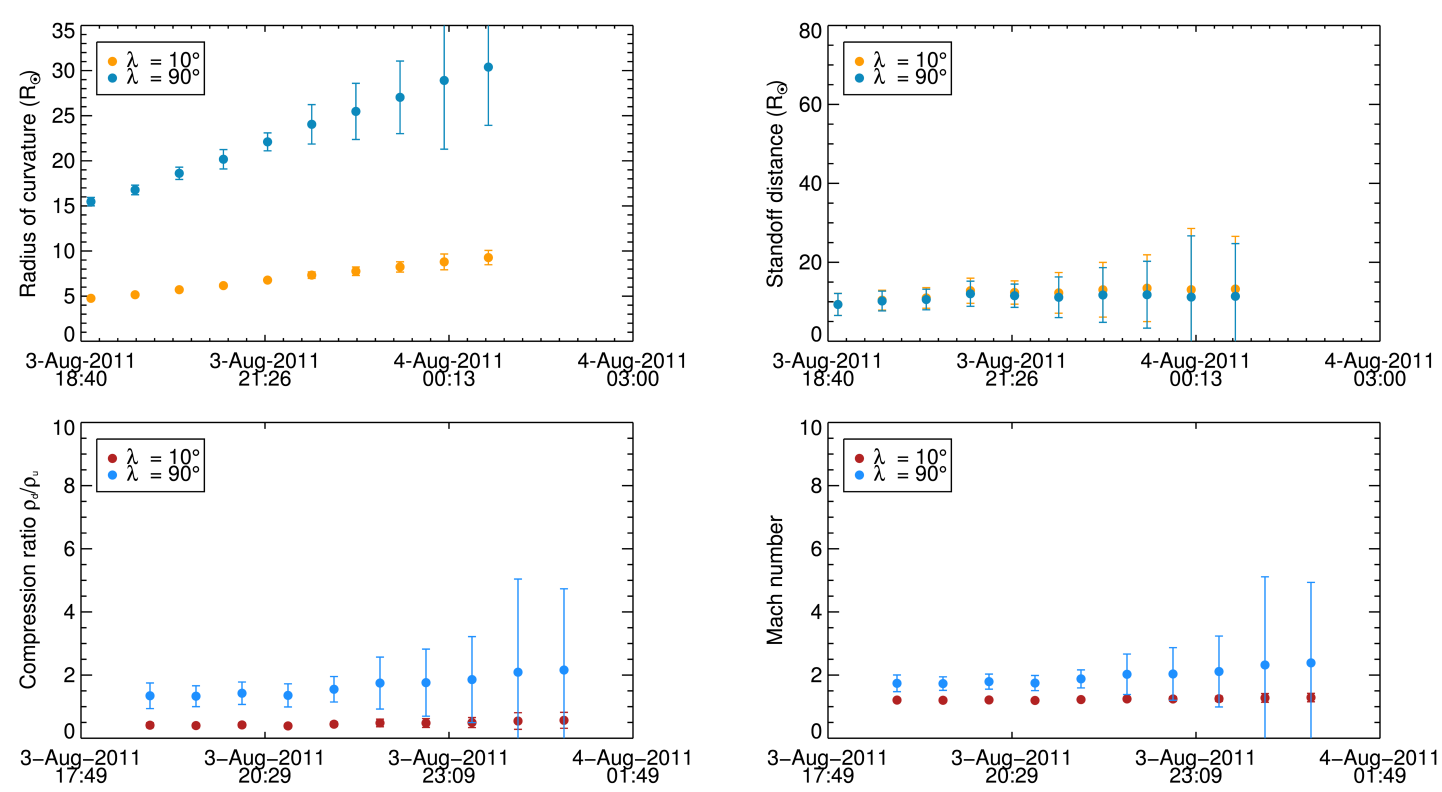

Figure 8.28.: Time evolution of the CME radius of curvature, the shock standoff distance, the compression ratio and the Mach number obtained applying the SSSEM for $\lambda=10^{\circ}$ and $\lambda=90^{\circ}$ to STEREO/SECCHI observations of the 03 August 2011 event.

\subsubsection{In-situ comparison}

The SSSEM results for the CME and shock kinematics as well as for the shock parameters were compared to in-situ data. The CME itself arrived at $L_{1}$ on 05 August 2011 at 03:30 UT. The shock arrival speed was about $500 \mathrm{~km} \mathrm{~s}^{-1}$. The CME and shock heights were fitted to a linear and a quadratic profile, and eruption time, arrival time and speed at $L_{1}$ were inferred and compared to observations.

Figure 8.29 shows ACE plasma and magnetic field data measured between 04 and 08 August 2011. A sudden increase in the solar wind parameters was recorded on 04

\begin{tabular}{|c|c|c|c|}
\hline & $\mathrm{EUVI}+\mathrm{ACE}$ & 1st degree fit $\lambda=0^{\circ}$ & 1st degree fit $\lambda=90^{\circ}$ \\
\hline CME eruption time & 03-Aug-2011 13:41 & 03-Aug-2011 12:38 & 03-Aug-2011 12:49 \\
\hline Shock arrival time & 04-Aug-2011 21:10 & 04-Aug-2011 23:33 & 05-Aug-2011 03:19 \\
\hline Shock arrival speed & $500 \mathrm{~km} \mathrm{~s}^{-1}$ & $1147 \mathrm{~km} \mathrm{~s}^{-1}$ & $1026 \mathrm{~km} \mathrm{~s}^{-1}$ \\
\hline CME arrival time & 05-Aug-2011 03:30 & 05-Aug-2011 05:28 & 05-Aug-2011 06:47 \\
\hline & $\mathrm{EUVI}+\mathrm{ACE}$ & 2nd degree fit $\lambda=0^{\circ}$ & 2nd degree fit $\lambda=90^{\circ}$ \\
\hline CME eruption time & 03-Aug-2011 13:41 & 03-Aug-2011 12:41 & 03-Aug-2011 12:50 \\
\hline Shock arrival time & 04-Aug-2011 21:10 & 05-Aug-2011 00:49 & 05-Aug-2011 04:20 \\
\hline Shock arrival speed & $500 \mathrm{~km} \mathrm{~s}^{-1}$ & $1043 \mathrm{~km} \mathrm{~s}^{-1}$ & $968 \mathrm{~km} \mathrm{~s}^{-1}$ \\
\hline CME arrival time & 05-Aug-2011 03:30 & 05-Aug-2011 07:53 & 05-Aug-2011 09:43 \\
\hline
\end{tabular}

Table 8.12.: Observed CME eruption time, arrival time and speed at $L_{1}$ compared to those obtained via a linear (top panel) and quadratic (bottom panel) fit to the CME height-time profile derived by applying the SSSEM for $\lambda=0^{\circ}$ and $\lambda=90^{\circ}$ for the 03 August 2011 event. 


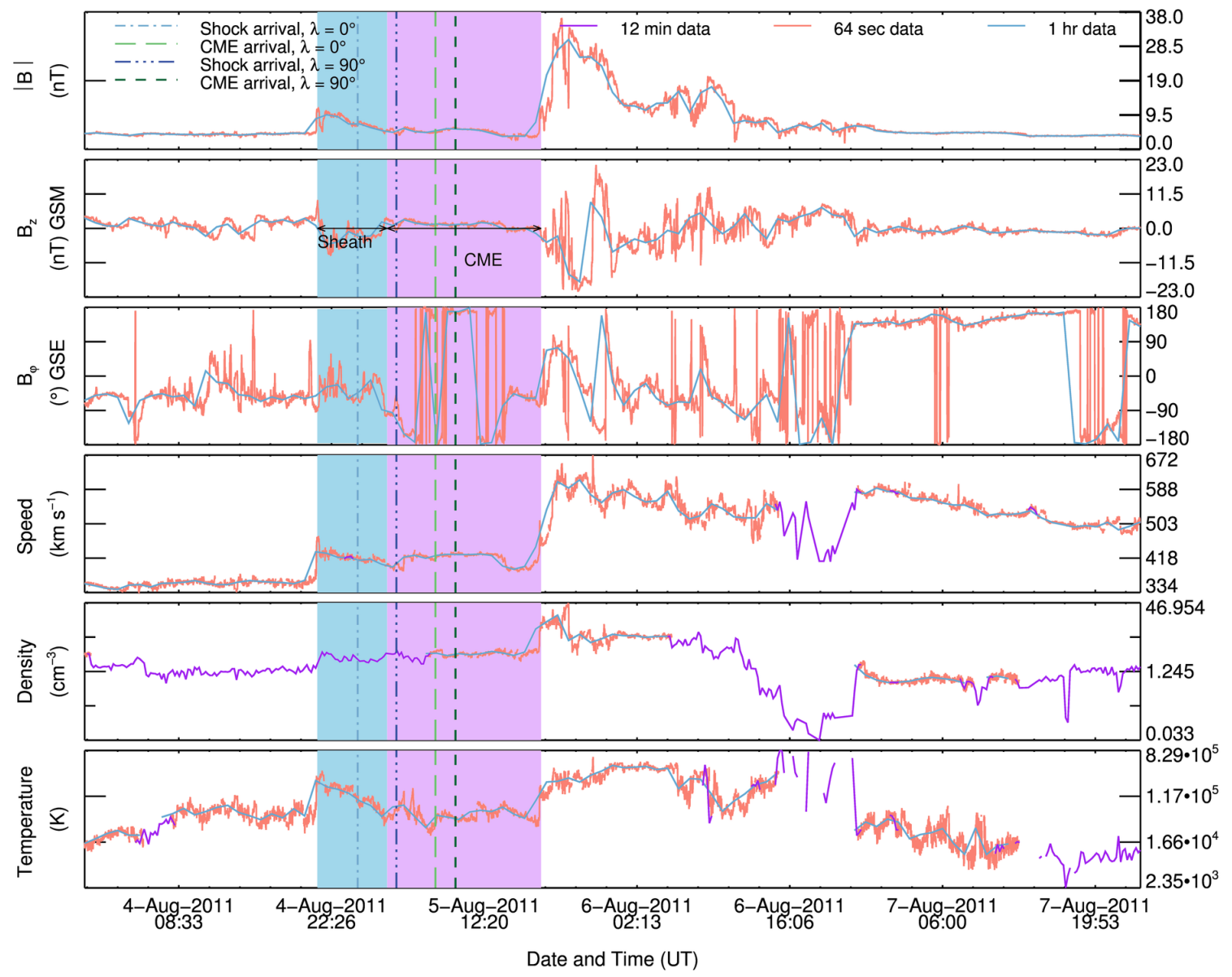

Figure 8.29.: ACE in-situ plasma and magnetic field measurements between 04 and 08 August 2011. The blue area indicates the shock sheath, and the purple area marks the CME passage. Overplotted are the SSSEM arrival times for $\lambda=0^{\circ}$ and $\lambda=90^{\circ}$ obtained via a quadratic fit to the CME and shock SSSEM heights.

August 2011 at 21:10 UT as a consequence of the passage of the CME-driven shock. Table 8.12 illustrates the results. The best arrival time predictions correspond to the linear fit to the SSSEM height determined in the case $\lambda=0^{\circ}$, although the arrival speed is significantly higher than the observed one. The second degree fit for $\lambda=0^{\circ}$ yields similar results. The $\lambda=90^{\circ}$ arrival times are later than the observed one, with discrepancies ranging from 6 to 8 hours in the worst case. Such errors are within the accuracy of the models employed.

The linear and quadratic fit to the CME and shock heights were also used to derive values for the shock parameters at $L_{1}$. These were compared to their observed counterparts obtained from ACE solar wind data. The comparison is shown in Table 8.13. The observed standoff distance, compression ratio, and Mach number were respectively $\Delta=15 R_{\odot}, \frac{\rho_{d}}{\rho_{u}}=1.89$, and $M=1.38$. The results for $\lambda=10^{\circ}$ yield a non-physical value of the compression ratio $\frac{\rho_{d}}{\rho_{u}}<1$ both for the linear and the quadratic fit. The best fit to observations corresponds to the value $\lambda=20^{\circ}$, for both propagation at constant 


\begin{tabular}{llll}
\hline & ACE & 1st degree fit $\lambda=10^{\circ}$ & 2nd degree fit $\lambda=10^{\circ}$ \\
\hline Standoff distance & $15 R_{\odot}$ & $26 R_{\odot}$ & $30 R_{\odot}$ \\
Compression ratio & 1.89 & 0.85 & 1.38 \\
Mach number & 1.38 & 1.44 & 0.74 \\
\hline & $\mathrm{ACE}$ & 1 st degree fit $\lambda=90^{\circ}$ & 2nd degree fit $\lambda=90^{\circ}$ \\
\hline Standoff distance & $15 R_{\odot}$ & $17 R_{\odot}$ & $24 R_{\odot}$ \\
Compression ratio & 1.89 & 0.78 & 3.26 \\
Mach number & 1.38 & -- & 4.31 \\
\hline
\end{tabular}

Table 8.13.: Comparison between standoff distance, compression ratio, and Mach number for the 03 August 2011 event as derived from ACE in-situ data, and from the linear and quadratic fit to the SSSEM heights for $\lambda=10^{\circ}$ and $\lambda=90^{\circ}$.

speed and constant deceleration. The associated shock parameters are $\Delta=23 R_{\odot}$, $\frac{\rho_{d}}{\rho_{u}}=1.71$, and $M=1.99$, and $\Delta=19 R_{\odot}, \frac{\rho_{d}}{\rho_{u}}=2.09$, and $M=2.32$ respectively.

\subsubsection{Corrections for off-Ecliptic propagation}

This section discusses the discrepancy between the standoff distance and the shock parameter calculated on the Ecliptic plane and at the CME apex (see Table 8.14) for the value of $\lambda$ corresponding to the best fits to the observations, i.e. $\lambda=20^{\circ}$. The radius of curvature seems to be the most affected value. Except for the Mach number derived via the quadratic fit, the standoff distance and the shock parameters on the Ecliptic plane differ from the ones at the CME apex by less than $25 \%$. Such results are as expected considering the non negligible CME angle of propagation with respect to the Ecliptic plane. Given the calculated errors, the SSSEM can nevertheless be considered a reliable method for the determination of the shock parameters for this event.

\begin{tabular}{lll}
\hline Relative error for & 1st degree fit $\lambda=20^{\circ}$ & 2nd degree fit $\lambda=20^{\circ}$ \\
\hline Standoff distance & $23 \%$ & $23 \%$ \\
CME curvature & $64 \%$ & $64 \%$ \\
Compression ratio & $25 \%$ & $25 \%$ \\
Mach number & $25 \%$ & $37 \%$ \\
\hline
\end{tabular}

Table 8.14.: Relative difference between the standoff distance, the CME radius of curvature, the compression ratio and the Mach number computed on the Ecliptic plane and at the CME apex for the 03 August 2011 event. The results for the value of lambda which yields the best fit to the in-situ shock parameters are shown.

The event analysed in this section erupted from NOAA AR 11261 on 03 August 2011. It was associated with a M6.0 flare, EUV dimmings, EUV waves and post eruptive 
loops.

Geometrical modelling via the GCS model was performed. The derived average speeds showed only a small decrease, from $1143 \mathrm{~km} \mathrm{~s}^{-1}$ between 15:08 and 18:49 UT to $818 \mathrm{~km} \mathrm{~s}^{-1}$ between 18:49 and 23:29 UT on 03 August 2011. Despite the difficulties in extending the COR2 fits to HI1 observations, the derived GCS apex heights are in good agreement with the SSSEM results.

The 03 August 2011 CME drove a shock whose signatures could be identified in both remote-sensing observations and time-elongation plots. The CME and shock kinematics were determined separately. The results indicate that both the CME and the shock propagated radially along the Sun-Earth line. The CME speed shows a roughly constant profile, whereas the shock speed profiles exhibit an initial acceleration, followed by deceleration and finally propagation at constant velocity.

An interplanetary shock was detected by ACE on 04 August 2011 at 21:10 UT, followed by the CME on 05 August 2011 at 03:30 UT. The interplanetary shock speed was $500 \mathrm{~km} \mathrm{~s}^{-1}$. Arrival time and speed predictions were obtained via a linear and a quadratic fit to the CME and shock SSSEM heights. The best fit to observations is found for $\lambda=0^{\circ}$ and propagation at constant speed, for which the arrival time estimates are only $\simeq 2.5$ hours later than the observed ones. The shock arrival speed is overestimated, with the predicted value being $v=1147 \mathrm{~km} \mathrm{~s}^{-1}$.

The temporal evolution of the shock parameters was obtained by combining the SSSEM results to models for the shock location. The standoff distance was found to slowly increase with time. The compression ratio and the Mach number, on the other hand, do not strongly vary with time. The case $\lambda=10^{\circ}$ yields non-physical values for the density compression ratio $\frac{\rho_{d}}{\rho_{u}}<1$.

The shock parameters were extrapolated to $L_{1}$ and compared to the values derived from ACE measurements, namely $\Delta=15 R_{\odot}, \frac{\rho_{d}}{\rho_{u}}=1.89$, and $M=1.38$. The best prediction is obtained for $\lambda=20^{\circ}$ when the CME and the shock are assumed to propagate at constant speed. It yields $\Delta=23 R_{\odot}, \frac{\rho_{d}}{\rho_{u}}=1.71$, and $M=1.99$. 


\subsection{The 22 October 2011 CME}

\subsubsection{Solar source region}

The 22 October 2011 CME erupted from NOAA AR 11314, located at N27W87 on the solar disk. SDO EUV images (see Figure 8.30) show flaring activity and post eruptive loops. STEREO EUVI A $195 \AA$ shows a wave propagating in the NE quadrant. GOES reported a M1.3 flare peaking at 09:18 UT. At the time of CME eruption STEREO A and $B$ were at an angular separation from Earth respectively of $105^{\circ}$ and $100^{\circ}$. The CME, seen on the West limb from Earth, appears as backsided from the STEREO B perspective. It was first observed in COR2 at 10:54 UT. A previous CME, which
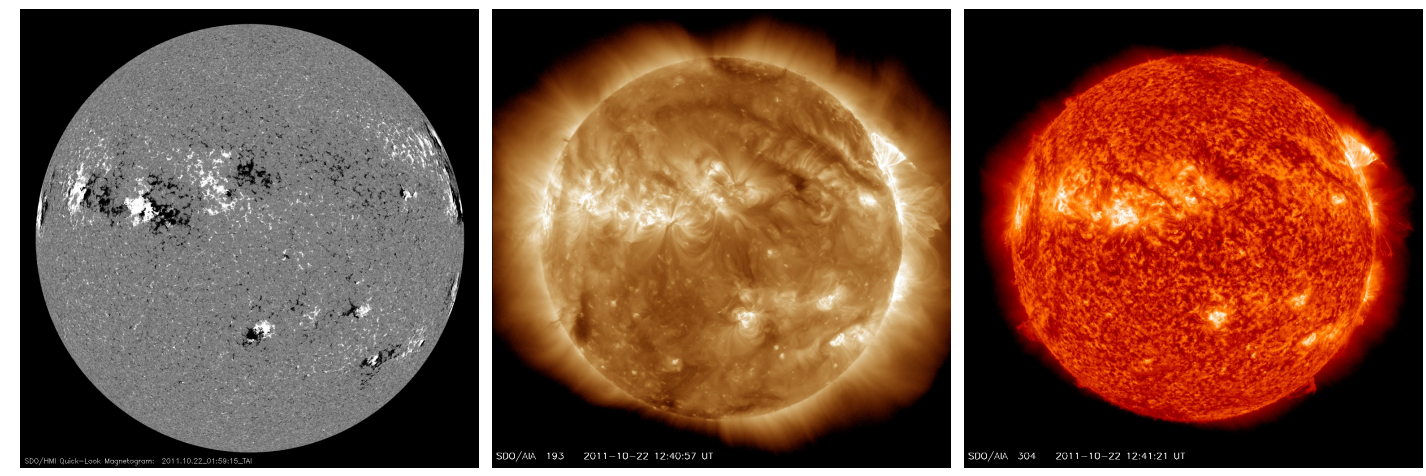

Figure 8.30.: SDO HMI, AIA $193 \AA$ and $304 \AA$ observations of the solar disk on 22 October 2011. Images courtesy of the NASA SDO consortium.

erupted on 21 October 2011 at 23:25 UT, is still visible in HI1 as the 22 October CME crosses the instrument's field of view at 13:49 UT. As the two CMEs propagate away from the Sun, it becomes increasingly difficult to differentiate their boundaries, to the point at which it is not really possible to distinguish the two events in HI2 images. Different stages of the CME interplanetary propagation are shown in Figure 8.31.

\subsubsection{GCS modelling}

The CME was modelled by applying the GCS model to STEREO COR2 and HI1 images. The COR2 modelling parameters are available in the AFFECTS database, and the corresponding GCS flux rope was extended to HI1 observations. Table 8.15 illustrates the results. The extension to HI1 observations was not straightforward due to the presence of a previously erupted CME in the imagers' field of view. This, together with the high inclination of the CME axis with respect to the Ecliptic plane ${ }^{13}$, makes the identification of the CME leading edge difficult, especially in the later propagation phase. The modelling results show that the CME propagation speed decreased from an average value of $2005 \mathrm{~km} \mathrm{~s}^{-1}$ between 13:08 and 16:49 UT to an average of $1044 \mathrm{~km} \mathrm{~s}^{-1}$ between 16:49 and 20:49 UT. This corresponds to an average acceleration

\footnotetext{
13i.e. the latitude of the CME apex, see Table 8.15.
} 

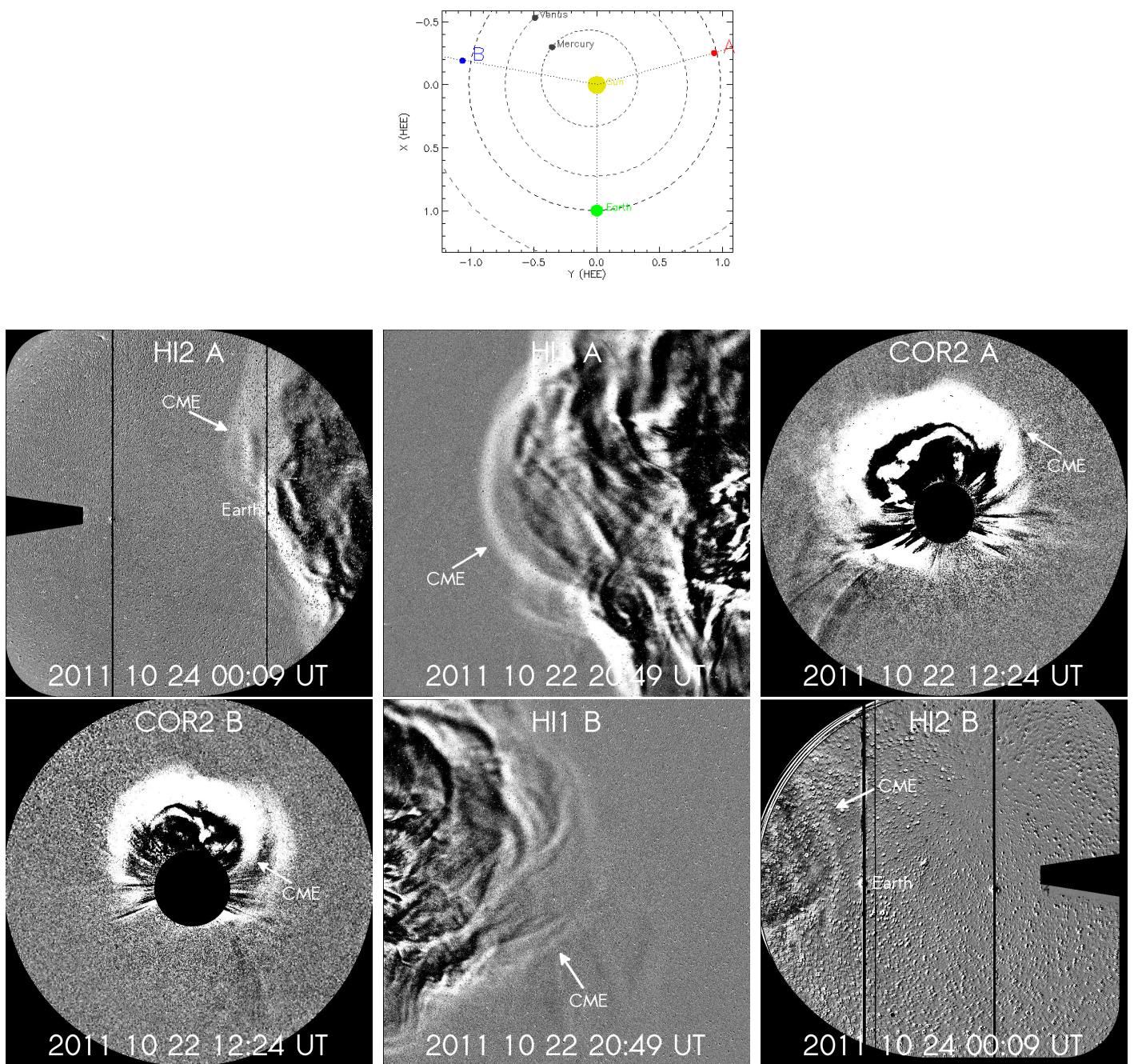

Figure 8.31.: Top panel: positions of the STEREO spacecraft on 22 October 2011. Image credit: NASA STEREO consortium. Bottom panel: difference images obtained from STERE$\mathrm{O} /$ SECCHI A and B COR2 HI1 and HI2 observations, showing subsequent phases of the CME evolution for the 22 October 2011 event.

of $-34.76 \mathrm{~m} \mathrm{~s}^{-2}$. The GCS radial width ${ }^{14}$ increased quite dramatically from a value of $2.19 R_{\odot}$ to over $38 R_{\odot}$ in less than 8 hours. The SSSEM heights do not compare exceptionally well to the ones obtained with the GCS. We find $34 \pm 3 R_{\odot}$ on 22 October 2011 at 16:49 UT and $48 \pm 3 R_{\odot}$ on 22 October 2011 at 16:49 UT for $\lambda=0^{\circ}$, and $33 \pm 3 R_{\odot}$ on 22 October 2011 at 16:49 UT and $47 \pm 3 R_{\odot}$ on 22 October 2011 at 16:49 $\mathrm{UT}$ for $\lambda=90^{\circ}$. The discrepancy is however unsurprising if we consider the $3 \mathrm{D}$ direction of propagation of the CME, highly off-Ecliptic, and the fact that a previous event is present in the HI1 field of view, making the identification of the CME boundaries rather uncertain.

\footnotetext{
${ }^{14}$ The radius of the GCS cross-section at the CME apex, $a=k R_{G C S}$.
} 

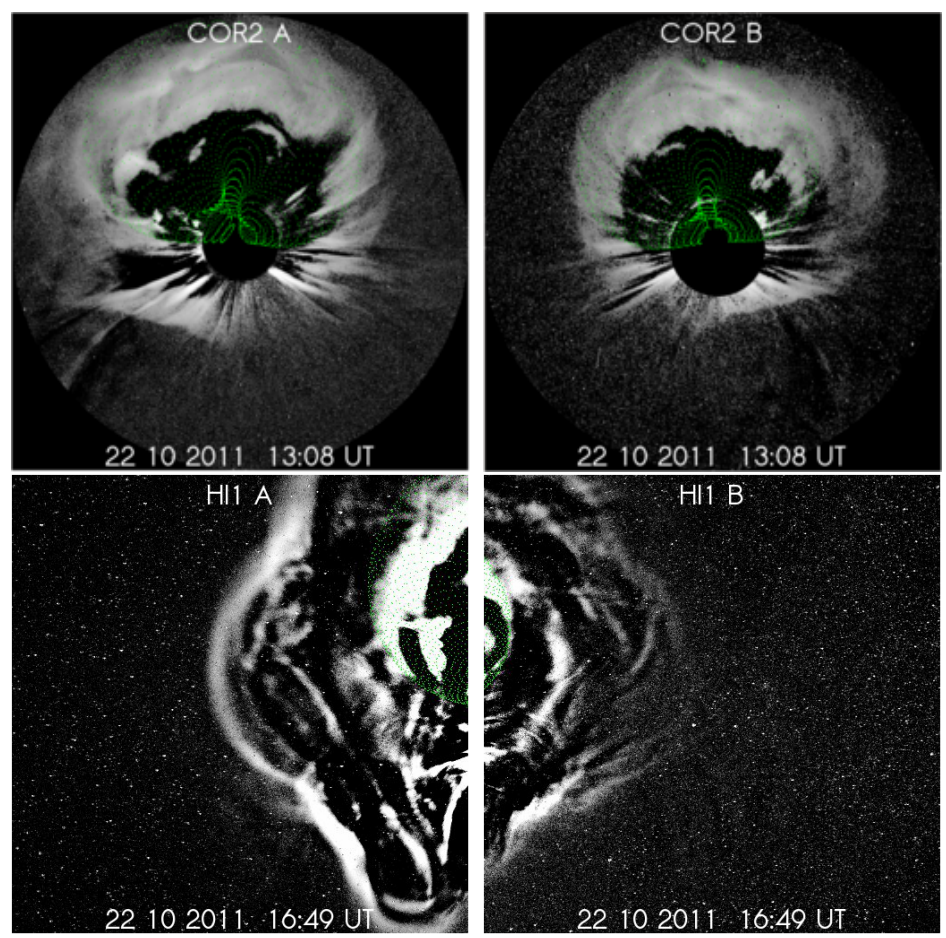

Figure 8.32.: GCS modelling for STEREO/SECCHI COR2 (upper panel) and HI1 (lower panels) for observations of the 22 October $2011 \mathrm{CME}$ at 13:08 UT and 16:49 UT.

\begin{tabular}{|r|l|l|l|l|r|l|l|l|}
\hline Instrument & Time & Lon & Lat & Tilt & Height & $\mathrm{k}$ & $\alpha$ & $\mathrm{a}$ \\
\hline COR2 & 22 -Oct-2011 13:08:00 & $59.25^{\circ}$ & $44.72^{\circ}$ & $-6.151^{\circ}$ & $6.64 R_{\odot}$ & 0.58 & 45.84 & $3.85 R_{\odot}$ \\
HI1 & $22-$ Oct-2011 16:49:00 & $59.25^{\circ}$ & $44.72^{\circ}$ & $-6.151^{\circ}$ & $44.88 R_{\odot}$ & 0.58 & 45.84 & $26.03 R_{\odot}$ \\
HI1 & $22-$ Oct-2011 20:49:00 & $59.25^{\circ}$ & $44.72^{\circ}$ & $-6.151^{\circ}$ & $66.50 R_{\odot}$ & 0.58 & 45.84 & $38.57 R_{\odot}$ \\
\hline
\end{tabular}

Table 8.15.: GCS modelling results for the 22 October 2011 CME. The table shows the imaging instrument, the time of observations, and the GCS parameters: Carrington longitude, heliographic latitude and tilt angle of the solar source region, flux rope height $R_{G C S}$, aspect ratio $k$, half-angle $\alpha$, and radial width $a=k R_{G C S}$.

\subsubsection{SSSEM results and in-situ comparison}

The top panel of Figure 8.33 shows the time-elongation plots obtained from STEREO/SECCHI A and B observations between 22 and 27 October 2011, which follow the evolution of solar wind transients along the Ecliptic plane. The green dots represent the elongations for the event under study. A large gap in the measured data points is present for observations close to the outer edge of the HI1 field of view, due to the faint signatures of the CME at those locations. The previous CME, which erupted on 21 October 2011 at 23:25 UT, on the other hand, presents strong signatures in the j-maps. As mentioned in Section 8.5.1, the 22 October 2011 CME reaches the previous one at the heights imaged by HI1. Indications of CME interaction are identifiable in the $\mathrm{j}$-maps in the form of distortions in the corresponding paths (see, e.g. Liu et al. 2012). Although it is difficult to distinguish the two events in HI2 observations, their 

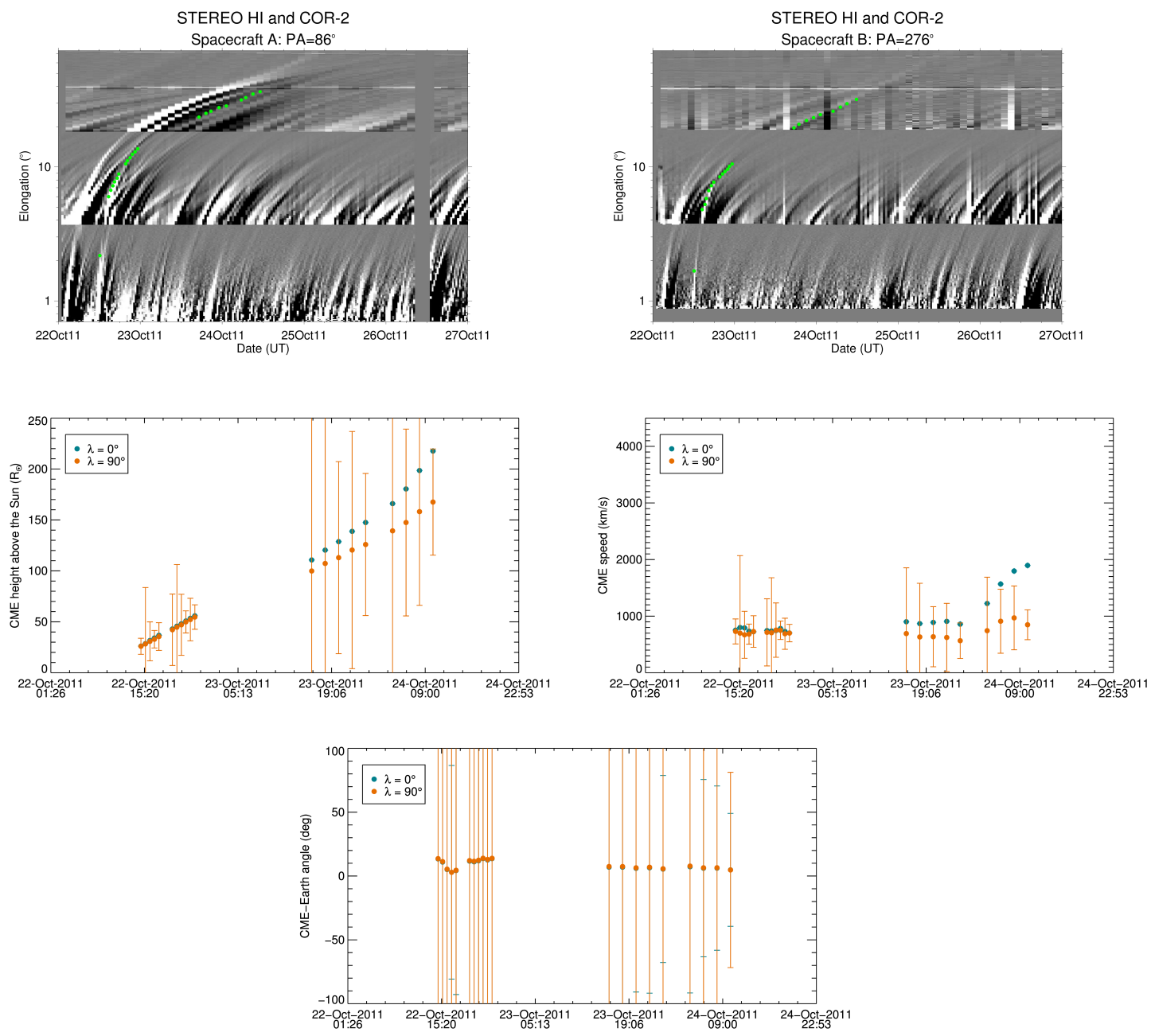

Figure 8.33.: Top panel: j-maps obtained from STEREO A (left) and B (right) observations between 22 and 27 October 2011. The green dots represent the measured elongations used to derive the CME kinematics. Bottom panel: temporal evolution of the CME height, speed and direction of propagation towards Earth obtained by applying the SSSEM for $\lambda=0^{\circ}$ and $\lambda=90^{\circ}$ to STEREO/SECCHI observations of the 22 October 2011 event.

tracks in the $\mathrm{j}$-maps remain identifiable even at those elongations. The propagation of the 22 October 2011 CME could therefore be followed up to very large heliocentric distances, of the order of $\simeq 200 R_{\odot}$. The CME kinematics was obtained by applying the SSSEM to the measured time-elongation pairs in the range $\lambda=0^{\circ}$ to $\lambda=90^{\circ}$. The results are shown in the bottom panel of Figure 8.33.

The plots show the temporal evolution of the CME height above the Sun, direction of propagation with respect to the Sun-Earth line, and speed. The gaps in the plots correspond to the missing elongation measurements in the j-maps. The blue dots correspond to results obtained for $\lambda=0^{\circ}$ whereas the orange dots represent the results obtained for $\lambda=90^{\circ}$. For small elongation angles the results for the two values of $\lambda$ are quite similar. At heliocentric distances above $\simeq 100 R_{\odot}$, on the other hand, the 


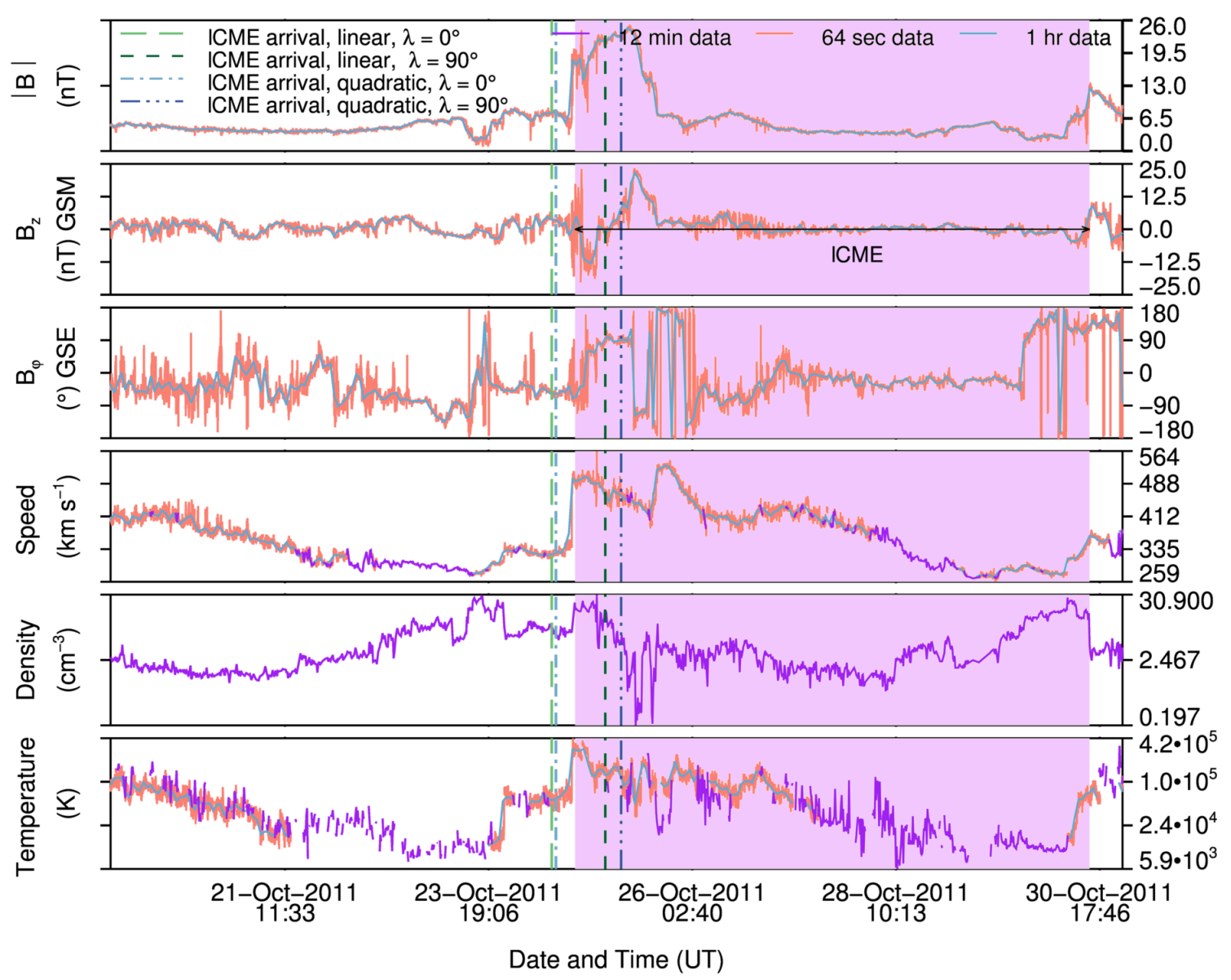

Figure 8.34.: ACE in-situ plasma and magnetic field measurements between 21 and 30 October 2011. The salmon area marks the CME passage. Overplotted are the SSSEM arrival times for $\lambda=0^{\circ}$ and $\lambda=90^{\circ}$ obtained via linear and quadratic fit to the SSSEM height for the 22 October 2011 event.

predicted distances for $\lambda=0^{\circ}$ are higher, and so is the speed, which also increases. The direction of propagation with respect to the Sun-Earth line, on the other hand, is very similar in both cases, and compatible with propagation towards Earth. The average values of the propagation angle are $\delta_{E}=8^{\circ} \pm 4^{\circ}$ for $\lambda=0^{\circ}$ and $\delta_{E}=9 \pm 4^{\circ}$ for $\lambda=90^{\circ}$. It is important to notice that, due to the source region location, it is likely that the feature tracked in the time-elongation plots is the CME flank rather than its apex. Linear and quadratic fits to the CME height were performed in order to derive arrival times and speed at $L_{1}$. Table 8.16 shows a comparison of the extrapolated arrival time and speed to in-situ data gathered by ACE at the same location. A sudden enhancement in the plasma parameters was detected on 24 October 2011 at 18:40 UT and corresponds to the CME-driven shock passage through the spacecraft. It is unclear though whether this is associated to the event under study, the previous CME, or a structure arising from a merger of the two. CME signatures lacking the classical magnetic cloud signature follow the shock passage. The CME and shock sys- 


\begin{tabular}{llll}
\hline & EUVI+ACE & 1st degree fit $\lambda=0^{\circ}$ & 1st degree fit $\lambda=90^{\circ}$ \\
\hline CME eruption time & $22-O c t-2011$ 10:26 & 22-Oct-2011 11:59 & 22-Oct-2011 07:17 \\
ICME arrival speed & $500 \mathrm{~km} \mathrm{~s}^{-1}$ & $853 \mathrm{~km} \mathrm{~s}^{-1}$ & $610 \mathrm{~km} \mathrm{~s}^{-1}$ \\
ICME arrival time & 24-Oct-2011 18:40 & 24-Oct-2011 12:19 & 25-Oct-2011 02:54 \\
\hline & & & \\
\hline & EUVI+ACE & 2nd degree fit $\lambda=0^{\circ}$ & 2nd degree fit $\lambda=90^{\circ}$ \\
\hline CME eruption time & $22-O c t-2011 ~ 10: 26$ & 22-Oct-2011 11:46 & 22-Oct-2011 09:08 \\
ICME arrival speed & $500 \mathrm{~km} \mathrm{~s}^{-1}$ & 741 $\mathrm{km} \mathrm{s}^{-1}$ & $469 \mathrm{~km} \mathrm{~s}^{-1}$ \\
ICME arrival time & 24-Oct-2011 18:40 & 24-Oct-2011 13:27 & 25-Oct-2011 07:14 \\
\hline
\end{tabular}

Table 8.16.: Observed CME eruption time, arrival time and speed at $L_{1}$ compared to those obtained via a linear (top panel) and quadratic (bottom panel) fit to the CME height-time profile derived applying the SSSEM for $\lambda=0^{\circ}$ and $\lambda=90^{\circ}$.

tem as observed in-situ are indicated in Figure 8.34 by the purple shaded area. The CME-driven shock crossed the ACE spacecraft on 24 October 2011 at 18:40 UT with a speed of about $500 \mathrm{~km} \mathrm{~s}^{-1}$. The $\lambda=0^{\circ}$ case predicts more accurate arrival times compared to the $\lambda=90^{\circ}$ case, although the latter yields better arrival speeds. The inclusion of deceleration marginally improves the arrival time determination for $\lambda=0^{\circ}$, but increases the discrepancy in the arrival times for $\lambda=90^{\circ}$. In the latter case, in fact, the predicted CME arrival is later than the observed one, and deceleration delays the arrival even further. A non-linear temporal evolution of the CME height is nevertheless to be expected to some extent due to the interaction with the previous event. The velocity profile for the $\lambda=0^{\circ}$ case indeed shows an increase in the later stages of CME propagation. However, this has been observed even in cases of single CMEs, and should be considered an artefact of the model rather than a signature of CME-CME interaction.

The event presented in this section erupted on 22 October 2011 from AR 11314. It was associated with a M1.3 flare, EUV waves and post-eruptive loops.

GCS modelling was performed, and it showed that the CME decelerated during its propagation. The extension was however not straightforward because of the presence of a previous CME in the images and of the large inclination of the CME axis with respect to the Ecliptic plane. The derived SSSEM heights are not in exceptional agreement with the GCS height, with errors as high as $\simeq 37 \%$. The large discrepancy is related to the fact that the GCS and the SSSEM followed the propagation of very different features within the CME for this event.

COR2 images show signatures of a CME-driven shock. Such signatures are not identifiable in HI1 images because of the presence of a formerly erupted CME erupted in the instrument's field of view. The analysis of the kinematics could not be performed 
separately for the CME and the shock. Evidence of CME-CME interaction can be identified in the time-elongation plots obtained for this event. The SSSEM results for the direction of propagation are compatible with a CME travelling towards Earth for both values of $\lambda$. The speed profiles show a constant behaviour up to roughly $150 R_{\odot}$. At larger distances the $\lambda=0^{\circ}$ speed increases quite rapidly with time. While this could be interpreted as evidence for CME-CME interaction, the more reasonable hypothesis is that the apparent acceleration is just an effect of the employed model.

ACE in-situ measurements show the passage of an interplanetary shock on 24 October 2011 at 18:40 UT, with speed $v \simeq 500 \mathrm{~km} \mathrm{~s}^{-1}$. The shock is followed by the CME, which lacks the classic magnetic cloud signatures. Arrival times and speeds at $L_{1}$ are derived from the SSSEM heights by assuming propagation at constant speed and at constant deceleration. The comparison with ACE measurements shows that the best arrival time estimates are associated to the $\lambda=0^{\circ}$ case when deceleration is assumed, which predicts the arrival to be on 24 October 2011 at 13:27 UT with a speed of $741 \mathrm{~km} \mathrm{~s}^{-1}$. 


\subsection{The 19 January 2012 CME}

\subsubsection{Solar source region}

The 19 January 2012 CME originated from NOAA AR 11402, located in the NE quadrant (N32E22). SDO EUV images show flaring activity at this location, and GOES measured a M3.2 flare between 13:44 and 17:50 UT. EUV dimming and post arcade loops were associated to the eruption of the CME. The separation angle with Earth was
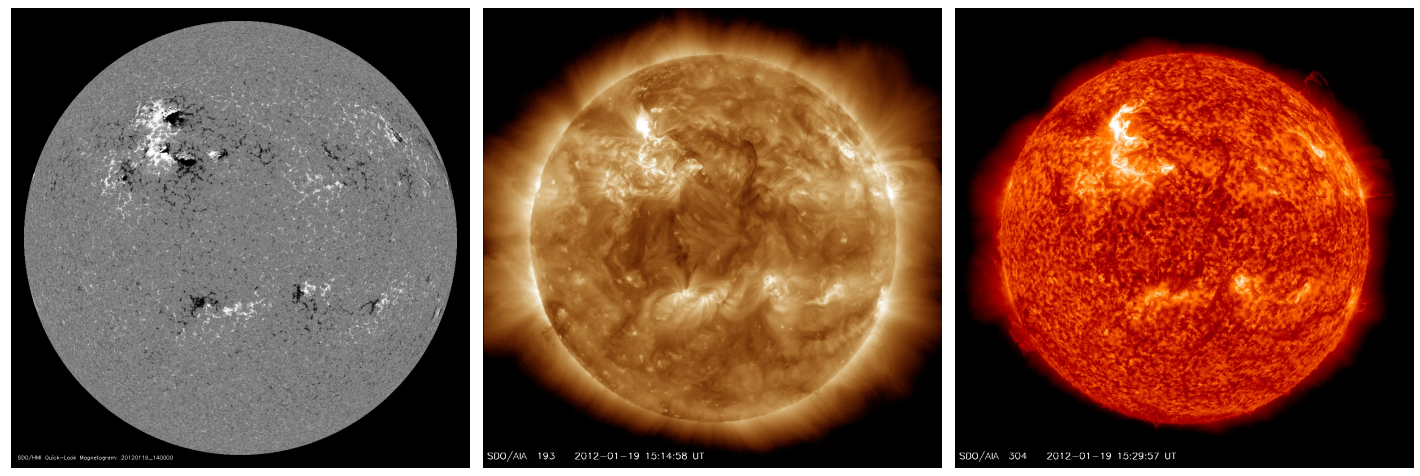

Figure 8.35.: SDO HMI, AIA $193 \AA$ and $304 \AA$ observations of the solar disk on 19 January 2012. Images courtesy of the NASA SDO consortium.

$107^{\circ}$ for STEREO A and $113^{\circ}$ for STEREO B. For this reason, given the position of the CME source region, the event appears as backsided from the STEREO A perspective, and as a limb event from STEREO B. The CME entered the COR1 A field of view on 19 January 2012 at 14:25 UT, and the COR1 B field of view at 14:45 UT. A smaller, fainter eruption was visible prior the expulsion of the event under study. The latter occults the former in later phases of their evolution. It is not clear though if this is just a projection effect, or if the two CMEs actually interacted. The CME was first observed by COR2 A and B at 15:24 UT. The deflected coronal streamers in COR2 observations are indicative of a shock being driven by the CME. Such hypothesis is supported by the presence of radio type II burst measured by Wind and most likely associated to this event (Liu et al., 2013). The CME first entered the field of view of HI1 on 19 January 2012 17:29 UT, and of HI2 on 20 January 2012 at 04:09 UT. Earth and Venus are visible in HI2 B observations.

\subsubsection{GCS modelling}

The CME was modelled in COR2 via the GCS model. The employed geometry seems to be able to represent the observed CME morphology, provided that the flux rope is distinguished from other features in the images. These include a previous CME, deflected streamers and density enhancements probably connected to a coronal shock. An extension to the HI1 field of view was attempted, and it is shown in the bottom panel of Figure 8.37.

The complex CME morphology, especially in the HI1 B field of view, makes it difficult to constrain the GCS parameters at these locations. The results are reported in Table 

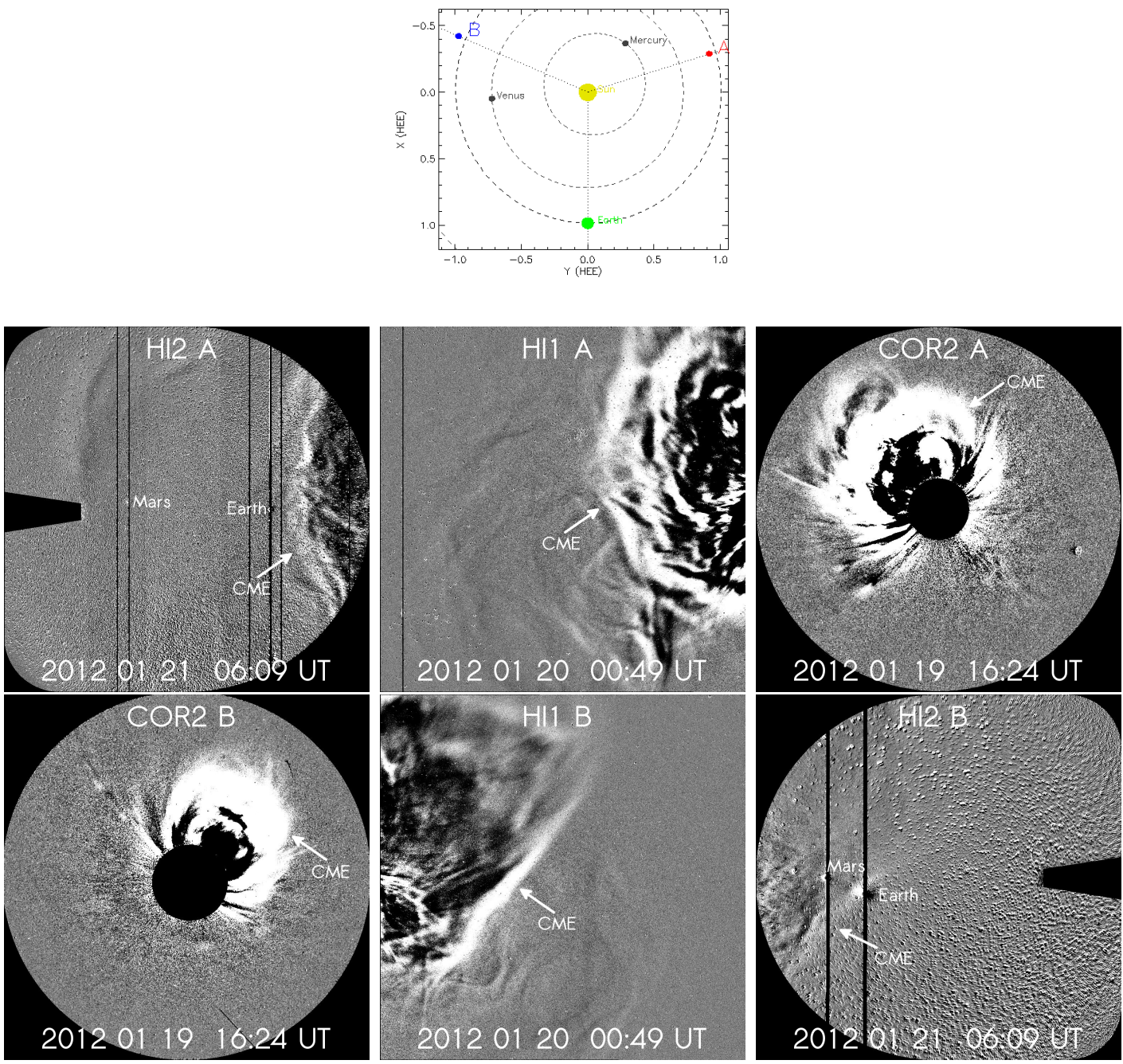

Figure 8.36.: Top panel: positions of the STEREO spacecraft on 19 January 2012. Image credit: NASA STEREO consortium. Bottom panel: difference images obtained from STEREO/SECCHI A and B COR2 HI1 and HI2 observations, showing subsequent phases of the CME evolution for the 19 January 2012 event. The top and bottom panel respectively show STEREO A and B images.

2 for completeness, and were driven mainly by the shape of the CME front in HI1 A images. However a definite statement about the CME geometry or whether it expanded self similarly at larger distances from the Sun can not be made based on these results.

\subsubsection{SSSEM results and in-situ comparison}

The results for the CME kinematics are shown in the bottom panel of Figure 8.38, where the CME height, speed and direction of propagation are plotted as a function of time. These were obtained by applying the SSSEM to time-elongation profiles manually measured from simultaneous STEREO A and B j-maps (top panel of Figure 8.38). 

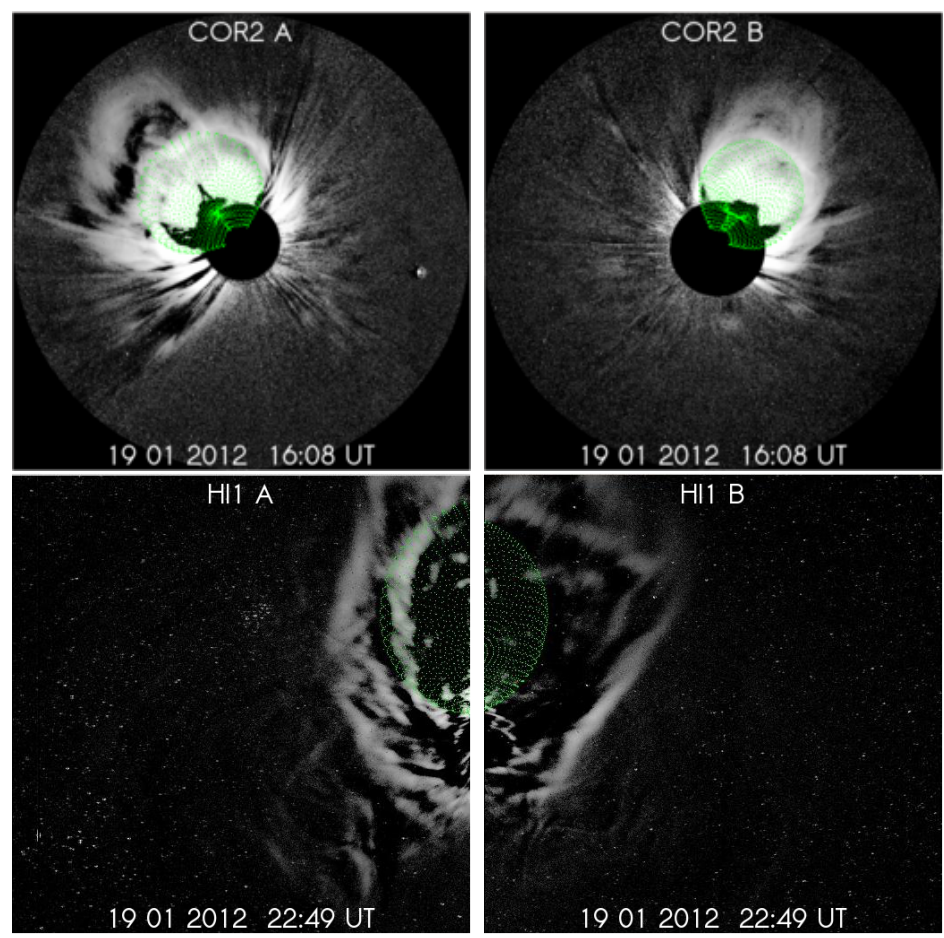

Figure 8.37.: GCS modelling for COR2 (upper panel) and HI1 (lower panels) for observations of the 19 January 2012 CME at 16:08 UT and 22:49 UT.

\begin{tabular}{|r|l|l|l|l|r|l|l|l|}
\hline Instrument & Time & Lon & Lat & Tilt & Height & $\mathrm{k}$ & $\alpha$ & $\mathrm{a}$ \\
\hline COR2 & 19-Jan-2012 16:08:00 & $231.43^{\circ}$ & $49.75^{\circ}$ & $-74.91^{\circ}$ & $8.35 R_{\odot}$ & 0.56 & 30.74 & $4.67 R_{\odot}$ \\
HI1 & 19-Jan-2012 22:49:00 & $231.43^{\circ}$ & $49.75^{\circ}$ & $-74.91^{\circ}$ & $38.43 R_{\odot}$ & 0.56 & 30.74 & $21.52 R_{\odot}$ \\
\hline
\end{tabular}

Table 8.17.: GCS modelling results for the 19 January 2012 CME. The table shows the imaging instrument, the time of observations, and the GCS parameters: Carrington longitude, heliographic latitude and tilt angle of the solar source region, flux rope height $R_{G C S}$, aspect ratio $k$, half-angle $\alpha$, and radial width $a=k R_{G C S}$.

The plots show the results derived for $\lambda=0^{\circ}$ and $\lambda=90^{\circ}$. The speed and height show similar profiles for distances up to roughly $80 R_{\odot}$, after which the $\lambda=0^{\circ}$ case predicts larger heights and increasing velocity. This apparent acceleration is purely an artefact of the model, as no interplanetary forces can explain such behaviour at those locations. The $\lambda=90^{\circ}$ speed profile, on the other hand, stays roughly constant during the CME propagation. The direction of propagation with respect to the Sun-Earth line begins with negative values, consistent with the source region longitude, located at $22^{\circ}$ East of the central meridian. The $\lambda=0^{\circ}$ profile starts at about $\delta_{E}=-25^{\circ}$, whereas the the $\lambda=90^{\circ}$ predicts initial values for the direction of propagation around $\delta_{E}=-45^{\circ}$. In both cases the results show a non-radial evolution, with the CME progressively drifting towards the Sun-Earth line. Liu et al. (2013) studied this event using the same techniques as those presented in this work, with results for the CME direction and height being compatible to the ones presented here. Their results for the CME speed show an 


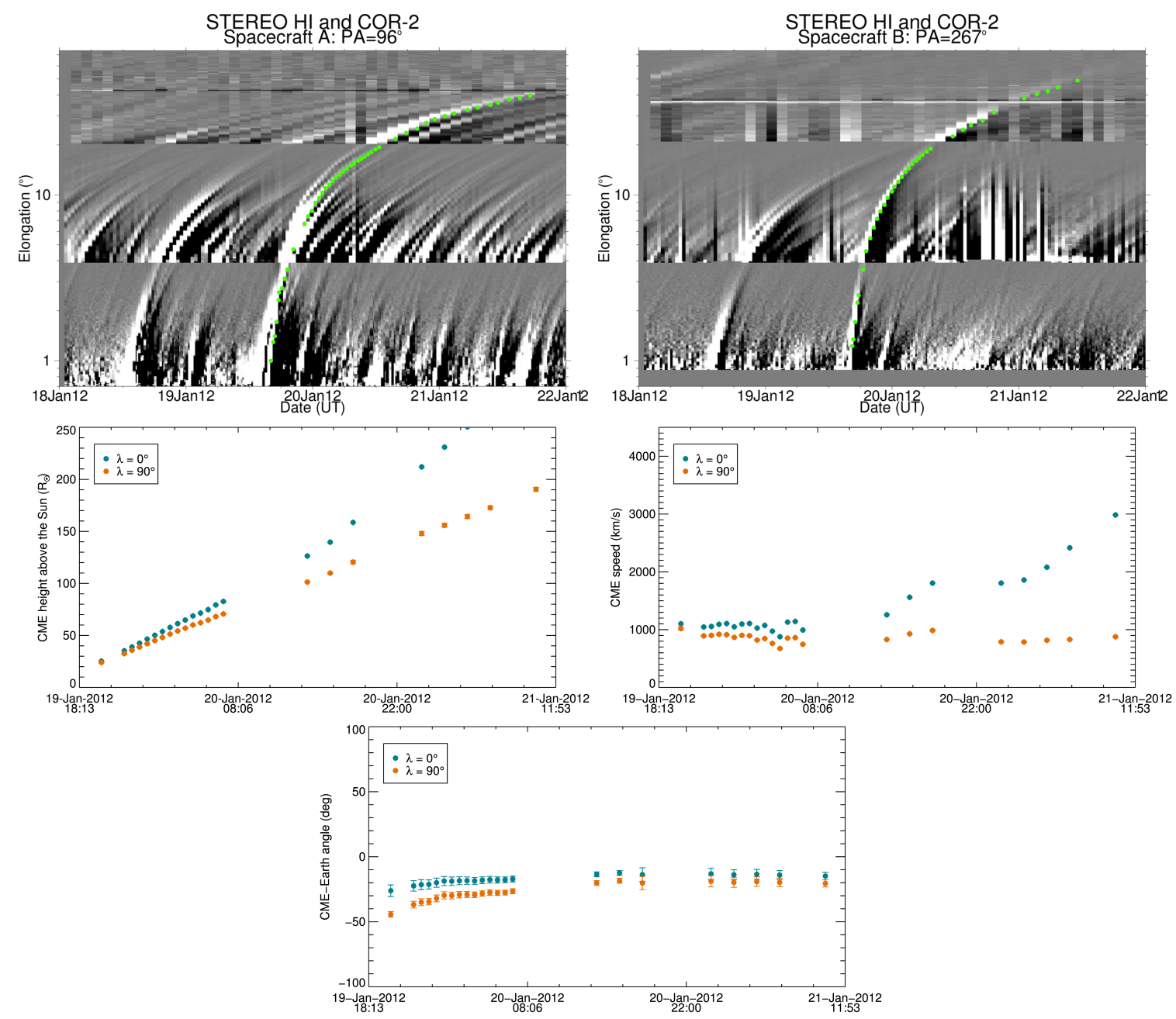

Figure 8.38.: Top panel: j-maps obtained from STEREO A (left) and B (right) observations between 18 and 22 January 2012. The green dots represent the measured elongations used to derive the CME kinematics. Bottom panel: temporal evolution of the CME height, speed and direction of propagation towards Earth obtained by applying the SSSEM for $\lambda=0^{\circ}$ and $\lambda=90^{\circ}$ to STEREO/SECCHI observations of the 19 January 2012 event.

initial acceleration phase followed by a deceleration between $15 R_{\odot}$ and $35 R_{\odot}$. This behaviour is not observed in the speed profile in Figure 8.38. The most likely reason for this difference is that no interpolation for the time-elongation profiles obtained from the $\mathrm{j}$-maps is performed in this work (see the introduction to this Chapter).

A linear and a quadratic fit to the SSSEM height allowed the computation of the eruption time and of the arrival time and speed at the location of ACE. In-situ plasma and magnetic field measurements are shown in Figure 8.39. An interplanetary shock associated with the CME was detected on 22 January 2012 at roughly 05:00 UT, followed by the CME on 23 January 2012 around 00:00 UT. The in-situ profiles of the magnetic field and plasma parameters do not resemble pure magnetic cloud signatures, however the lower temperature and density and the reduced variance of the magnetic field are indicative of CME passage across the spacecraft. A second shock, arriving at 


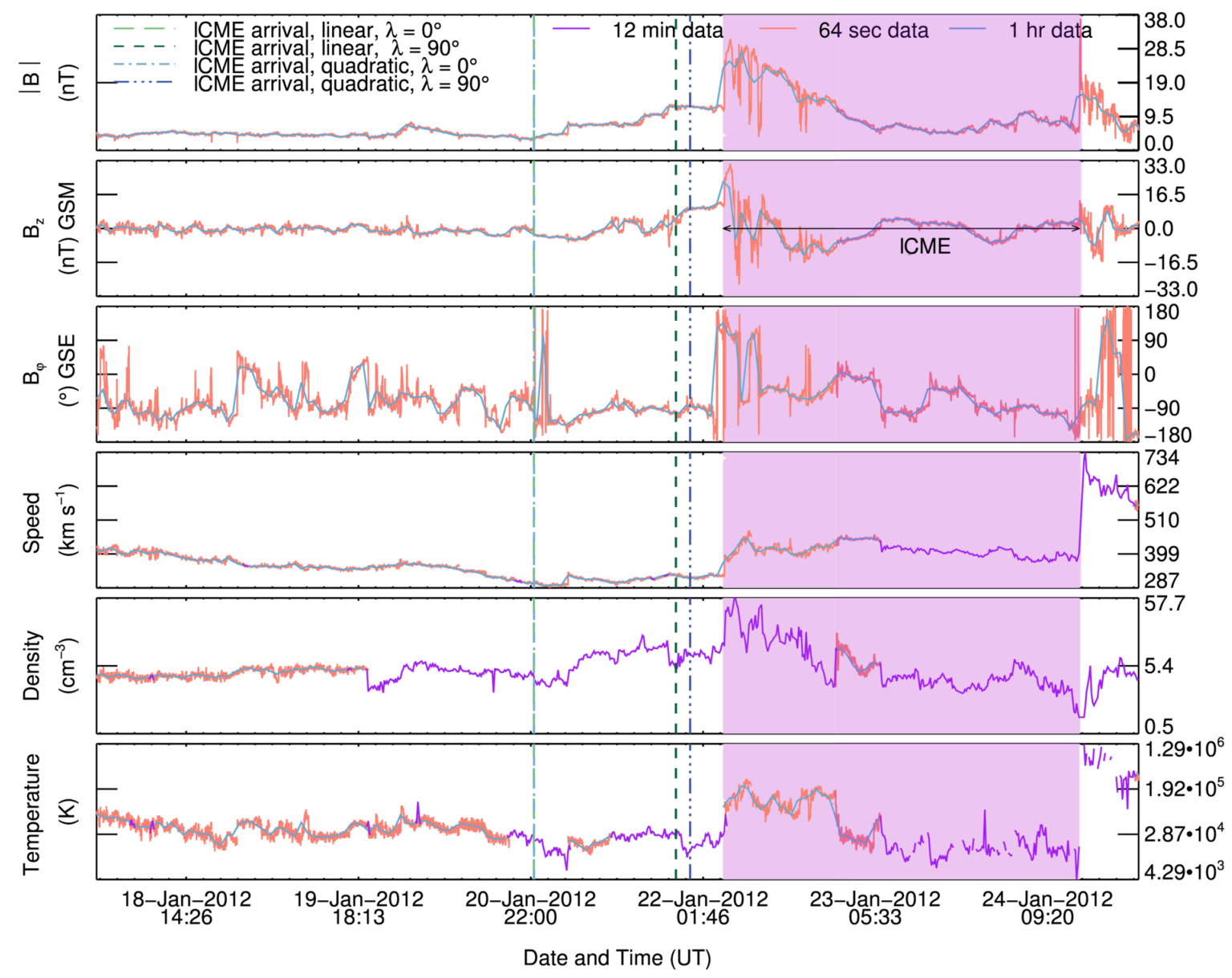

Figure 8.39.: ACE in-situ plasma and magnetic field measurements between 18 and 24 January 2012. The purple marks the ICME passage. Overplotted are the SSSEM arrival times for $\lambda=0^{\circ}$ and $\lambda=90^{\circ}$ obtained via linear and quadratic fit to the SSSEM height.

\begin{tabular}{llll}
\hline & EUVI+ACE & 1st degree fit $\lambda=0^{\circ}$ & 1st degree fit $\lambda=90^{\circ}$ \\
\hline CME eruption time & 19-Jan-2012 13:44 & 19-Jan-2012 19:51 & 19-Jan-2012 14:48 \\
CME arrival speed & $450 \mathrm{~km} \mathrm{~s}^{-1}$ & 1548 $\mathrm{km} \mathrm{s}^{-1}$ & $755 \mathrm{~km} \mathrm{~s}^{-1}$ \\
CME arrival time & 22-Jan-2012 03:00 & 20-Jan-2012 22:29 & 21-Jan-2012 21:24 \\
\hline & & & \\
\hline & EUVI+ACE & 2nd degree fit $\lambda=0^{\circ}$ & 2nd degree fit $\lambda=90^{\circ}$ \\
\hline CME eruption time & 19-Jan-2012 13:44 & 19-Jan-2012 21:52 & 19-Jan-2012 14:56 \\
CME arrival speed & 450 $\mathrm{km} \mathrm{s}^{-1}$ & 1628 $\mathrm{km} \mathrm{s}^{-1}$ & 709 $\mathrm{km} \mathrm{s}^{-1}$ \\
$\mathrm{CME}$ arrival time & 22-Jan-2012 03:00 & 20-Jan-2012 22:31 & 21-Jan-2012 23:41 \\
\hline
\end{tabular}

Table 8.18.: Observed CME eruption time, arrival time and speed at $L_{1}$ compared to those obtained via a linear (top panel) and quadratic (bottom panel) fit to the CME height-time profile derived by applying the SSSEM for $\lambda=0^{\circ}$ and $\lambda=90^{\circ}$ to STEREO/SECCHI observations of the 19 January 2012 event.

$L_{1}$ on 24 January 2012 at about 14:30 UT is associated with the CME which erupted on 23 January 2012 (see Section 8.7). The lack of magnetic cloud signatures in in-situ 
data and the observed highly off-Ecliptic position of the CME apex (see Figure 8.37) suggest that ACE was hit by the CME flanks. Table 8.18 shows a comparison between the observed and predicted eruption time, arrival time and arrival speed. The $\lambda=90^{\circ}$ case predicts more accurate arrival times, with the inclusion of deceleration reducing the arrival time difference to less that 3.5 hours. The speed extrapolation also yields better results for the $\lambda=90^{\circ}$ case compared to the results for $\lambda=0^{\circ}$. It overestimates the observed one by roughly $260 \mathrm{~km} \mathrm{~s}^{-1}$. A comparison between interplanetary (see Figure 8.38) and in-situ values of the CME speed 8.18 shows significant deceleration most likely due to solar wind drag.

The CME event analysed in this section erupted on 19 January 2012. It was associated with an M3.2 flare, EUV dimmings and post eruptive loops. GCS modelling shows that the GCS is appropriate in reproducing the CME morphology observed in COR2 images. However, the extension of the modelling to the HI1 field of view was not conclusive. HI1 images, in fact, only capture the CME flanks because of the highly inclined trajectory of the CME apex with respect to the Ecliptic plane. As a result the GCS parameters cannot be constrained at heliocentric distances above $15 R_{\odot}$, and whether the $C M E$ expanded self-similarly cannot be stated with certainty.

The deflected coronal streamers observed in COR2 images and the detection of radio type II bursts suggest the presence of a CME-driven shock. Such signatures, however, cannot be identified in HI1 observations nor in time-elongation plots. For this reason a separate analysis of the CME and shock kinematics was not possible for this event. The SSSEM was applied to time-elongation profiles obtained from STEREO A and B observations, and the temporal evolution of the CME height, direction of propagation an speed was derived. A comparison between ACE in-situ data and arrival time and speed extrapolations to $L_{1}$ show that the best accuracy is achieved for $\lambda=90^{\circ}$ when deceleration is assumed. The observed arrival time is 22 January 2012 at 03:00 UT, and the observed arrival speed is $450 \mathrm{~km} \mathrm{~s}^{-1}$. The second degree fit to the SSSEM height in the $\lambda=90^{\circ}$ case predicts the arrival time to be on 21 January 2012 at 23:41 UT, with a speed of $709 \mathrm{~km} \mathrm{~s}^{-1}$. 


\subsection{The 23 January 2012 CME}

\subsubsection{Solar source region}

The event presented in this Section erupted from NOAA AR 11402, source region of the 19 January 2012 as well (see Section 8.6). Because of solar rotation the position of the source region on the solar disk changed from N32E22 on 19 January 2012 to N29W20 on 23 January 2012. The event is made up of two subsequent events: composite EUVI $304 \AA$ and COR1 images show a first eruption at 02:26 UT associated to EUV dimming, followed by a large CME which appears in COR1 at 03:46 UT and seems to overtake the previous one. This was associated to long duration M8.7 X-ray flare between 03:38 and 04:34 UT, post eruptive arcades and a EUV wave.
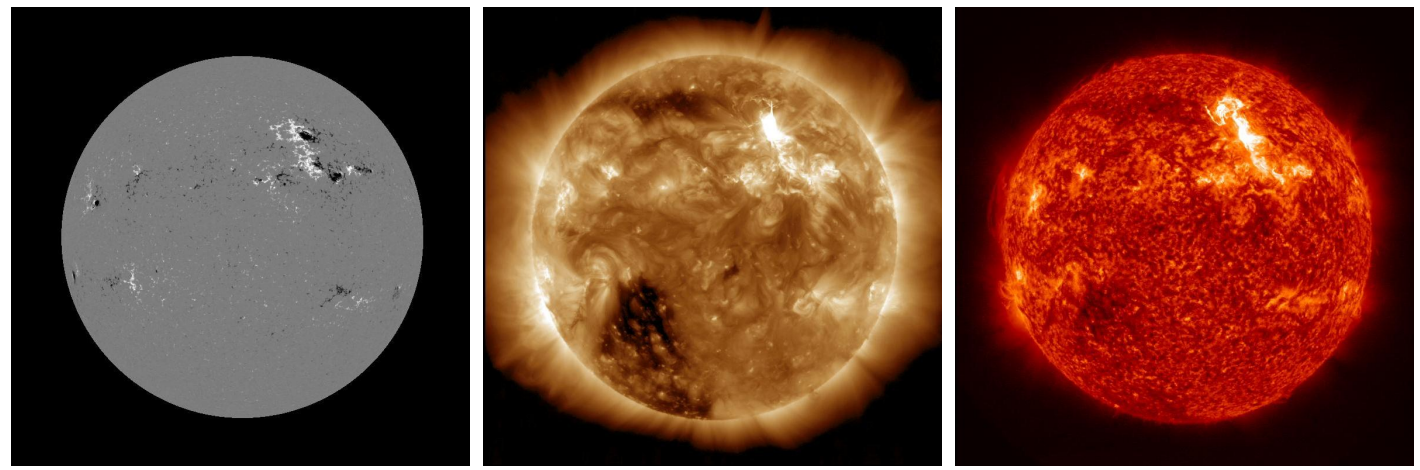

Figure 8.40.: SDO HMI, AIA $193 \AA$ and $304 \AA$ observations of the solar disk on 23 January 2012. Images courtesy of the NASA SDO consortium.

STEREO A and B were respectively at $107^{\circ}$ and $114^{\circ}$ away from the Sun-Earth line. The event is seen as backsided from STEREO B and as a limb event from STEREO A. It was first observed by COR2 A and B on 23 January 2012 at 03:24 UT, and it entered the HI1 A and B fields of view respectively on 23 January 2012 at 04:49 UT and 23 January 2012 at 05:29 UT. HI2 observations are available from 23 January 2012 at 16:09 UT. Indications of the presence of a CME-driven shock are found in COR2 observations. However a separate analysis of the CME and the shock kinematics could not be performed for this event due to the lack of shock signatures in HI1 images.

\subsubsection{GCS modelling}

The CME was fitted with the GCS geometry in COR2 and HI1 images. The fitting results are illustrated in Table 8.19. The flux rope model seems adequate to reproduce the morphology observed in COR2. The extension of the fit to HI1 images however presents issues, especially for HI1 B images. Due to the size and orientation of the $\mathrm{CME}$, which propagates at $25^{\circ}$ with respect to the Ecliptic plane, much of the CME itself falls outside the HI1 field of view. Although structures visible in COR2 can be identified in HI1 as well, the CME can be modelled with the GCS in HI1 only for the first few snapshots, when its size does not exceed the size of the imager's field of view. The GCS heights compare fairly well with the ones determined via the SSSEM. 

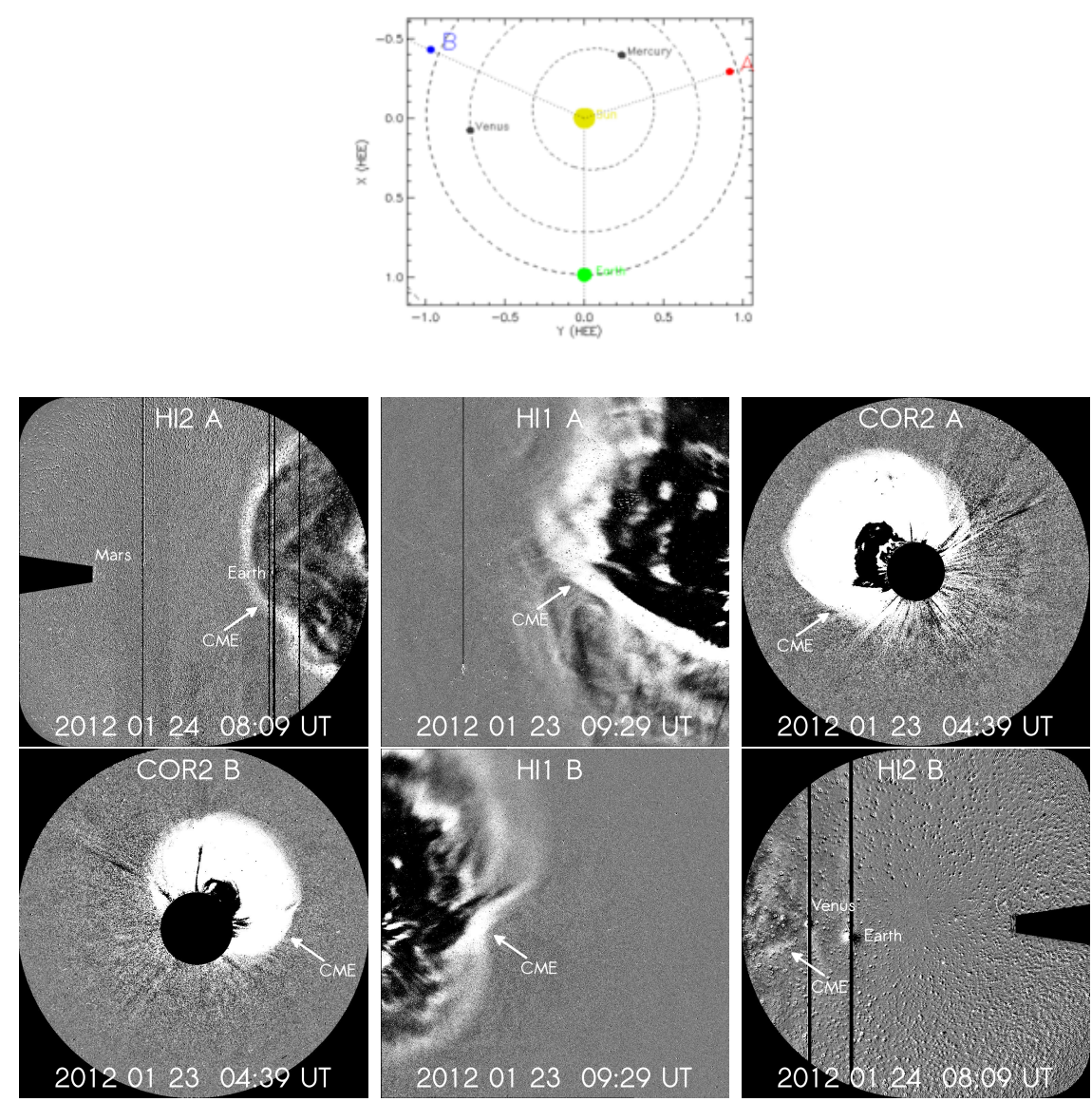

Figure 8.41.: Top panel: positions of the STEREO spacecraft on 23 January 2012. Image credit: NASA STEREO consortium. Bottom panel: difference images obtained from STEREO/SECCHI A (top panel) and B (bottom panel) COR2 HI1 and HI2 observations, showing subsequent phases of the CME evolution for the 23 January 2012 event. The top and bottom panel respectively show STEREO A and B images.

For $\lambda=0^{\circ}$ the CME heliocentric distances are $R_{S S S E M}=5 \pm 3 R_{\odot}$ at 04:24 UT, $R_{S S S E M}=12 \pm 3 R_{\odot}$ at 05:24 UT, and $R_{S S S E M}=25 \pm 3 R_{\odot}$ at 06:49 UT. For $\lambda=90^{\circ}$, on the other hand, $R_{S S S E M}=5 \pm 3 R_{\odot}$ at 04:24 UT, $R_{S S S E M}=11 \pm 3 R_{\odot}$ at 05:24 $\mathrm{UT}$, and $R_{S S S E M}=23 \pm 3 R_{\odot}$ at 06:49 UT. The derived GCS heights are higher than the SSSEM ones. This is somehow expected considering that the GCS height refers to the heliocentric distance of the CME apex, whereas the SSSEM yields the distance determined on the Ecliptic plane. The HI1 results show the biggest discrepancy. This is not surprising, given the difficulties in applying the GCS to HI images. The CME apex speed increased from an average of $1462 \mathrm{~km} \mathrm{~s}^{-1}$ between 04:08 UT and 05:08 UT to $2632 \mathrm{~km} \mathrm{~s}^{-1}$ between 05:08 UT and 06:49 UT, corresponding to an average acceleration of $102.55 \mathrm{~m} \mathrm{~s}^{-2}$. GCS modelling of the CME to higher distances (not shown) shows that it decelerated to an average speed of $985 \mathrm{~km} \mathrm{~s}^{-1}$ between 06:49 and 10:49 UT. The average acceleration between 04:08 UT and 10:49 UT was $-1.98 \mathrm{~m} \mathrm{~s}^{-2}$.This is consistent with the SSSEM results discussed in the next section. 

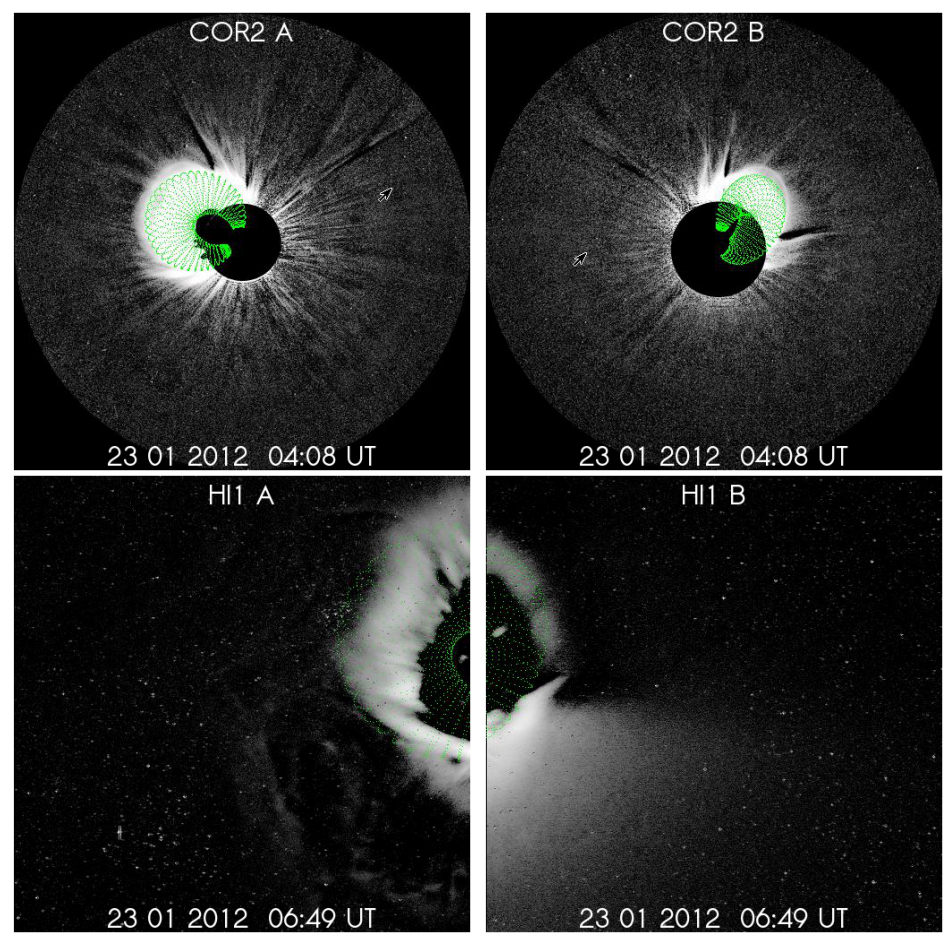

Figure 8.42.: GCS modelling for STEREO/SECCHI COR2 (upper panel) and HI1 (lower panels) for observations of the 23 January $2012 \mathrm{CME}$ at 04:08 UT and 06:49 UT.

\begin{tabular}{|r|l|l|l|l|r|l|l|r|}
\hline Instrument & Time & Lon & Lat & Tilt & \multicolumn{1}{l|}{ Height } & $\mathrm{k}$ & $\alpha$ & $\mathrm{a}$ \\
\hline COR2 & 23-Jan-2012 04:08:00 & $202.36^{\circ}$ & $25.71^{\circ}$ & $-68.20^{\circ}$ & $6.64 R_{\odot}$ & 0.33 & 55.91 & $2.19 R_{\odot}$ \\
COR2 & 23-Jan-2012 05:08:00 & $202.36^{\circ}$ & $25.71^{\circ}$ & $-68.20^{\circ}$ & $15.14 R_{\odot}$ & 0.33 & 55.91 & $4.99 R_{\odot}$ \\
HI1 & 23-Jan-2012 06:49:00 & $202.36^{\circ}$ & $25.71^{\circ}$ & $-68.20^{\circ}$ & $38.08 R_{\odot}$ & 0.33 & 55.91 & $12.57 R_{\odot}$ \\
\hline
\end{tabular}

Table 8.19.: GCS modelling results for the 23 January 2012 CME. The table shows the imaging instrument, the time of observations, and the GCS parameters: Carrington longitude, heliographic latitude and tilt angle of the solar source region, flux rope height $R_{G C S}$, aspect ratio $k$, half-angle $\alpha$, and radial width $a=k R_{G C S}$.

\subsubsection{SSSEM results and in-situ comparison}

Time-elongations plots were derived for STEREO/SECCHI observations between 22 and 25 January 2012, and are shown in the top panel of Figure 8.43. The green dots represent the measured time-elongation pairs, which were used as an input to derive the CME kinematics via the application of the SSSEM. The results are shown in the bottom panel of Figure 8.43 for $\lambda=0^{\circ}$ and $\lambda=90^{\circ}$. Both cases show very similar results up to roughly $70 R_{\odot}$. At larger heliocentric distances the height and speed for the $\lambda=0^{\circ}$ case become higher than for $\lambda=90^{\circ}$, with the $\lambda=0^{\circ}$ speed seemingly indicative of acceleration. However, this is considered non-physical, since no forces can be invoked to explain the presence of acceleration at those distances from the Sun. The gap in the points is due to lack of simultaneous time-elongation measurements from $\mathrm{A}$ and $\mathrm{B}$ j-maps. The initial direction of propagation, $\delta_{E} \sim 15^{\circ}$ is compatible with the 

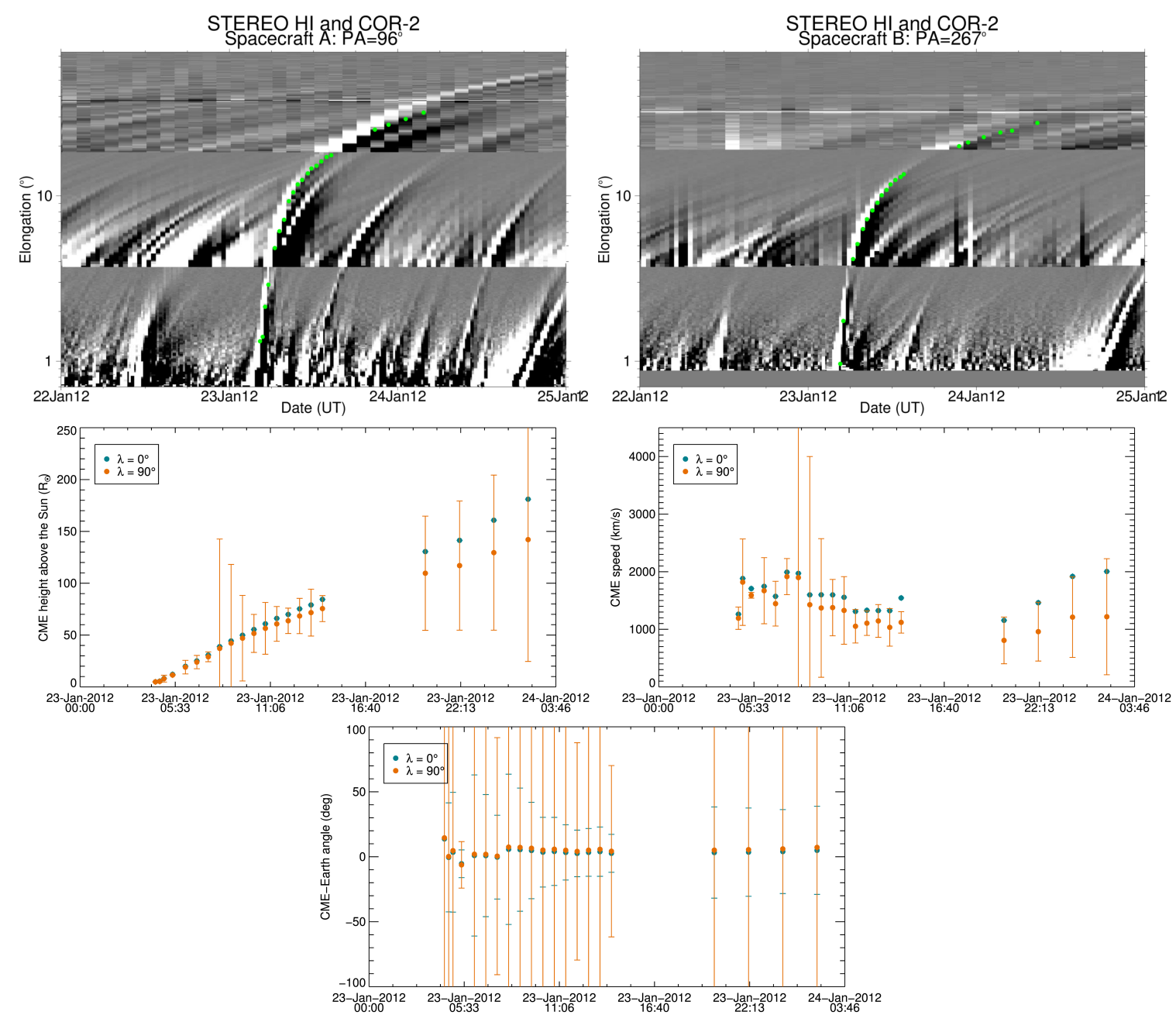

Figure 8.43.: Top panel: j-maps obtained from STEREO A (left) and B (right) observations between 22 and 25 January 2012. The green dots represent the measured elongations used to derive the CME kinematics. Bottom panel: temporal evolution of the CME height, speed and direction of propagation towards Earth obtained by applying the SSSEM for $\lambda=0^{\circ}$ and $\lambda=90^{\circ}$ to STEREO/SECCHI observations of the 23 January 2012 event.

source region longitude. It decreases towards angles smaller than $10^{\circ}$ as the CME moves outwards. The errors in the determined quantities are very large, especially for $\lambda=90^{\circ}$. This was found for different sets of time-elongation measurements, independent on the particular feature that is tracked in the j-maps. It is therefore likely that this is an effect of viewing angle and direction of propagation of the CME. The event was also studied by Liu et al. (2013), with similar results.

Figure 8.44 shows ACE plasma and magnetic field data between 22 and 28 January 2012. The passage of a shock associated with the CME investigated here is seen on 24 January 2012 at 14:30 UT in the sudden increase in density, velocity, temperature and magnetic field strength. The purple area in the plot shows what was interpreted as the shock and CME passage through ACE. The speed at the shock front was about $730 \mathrm{~km} \mathrm{~s}^{-1}$. No magnetic cloud signatures are observed in the data. According to Liu 


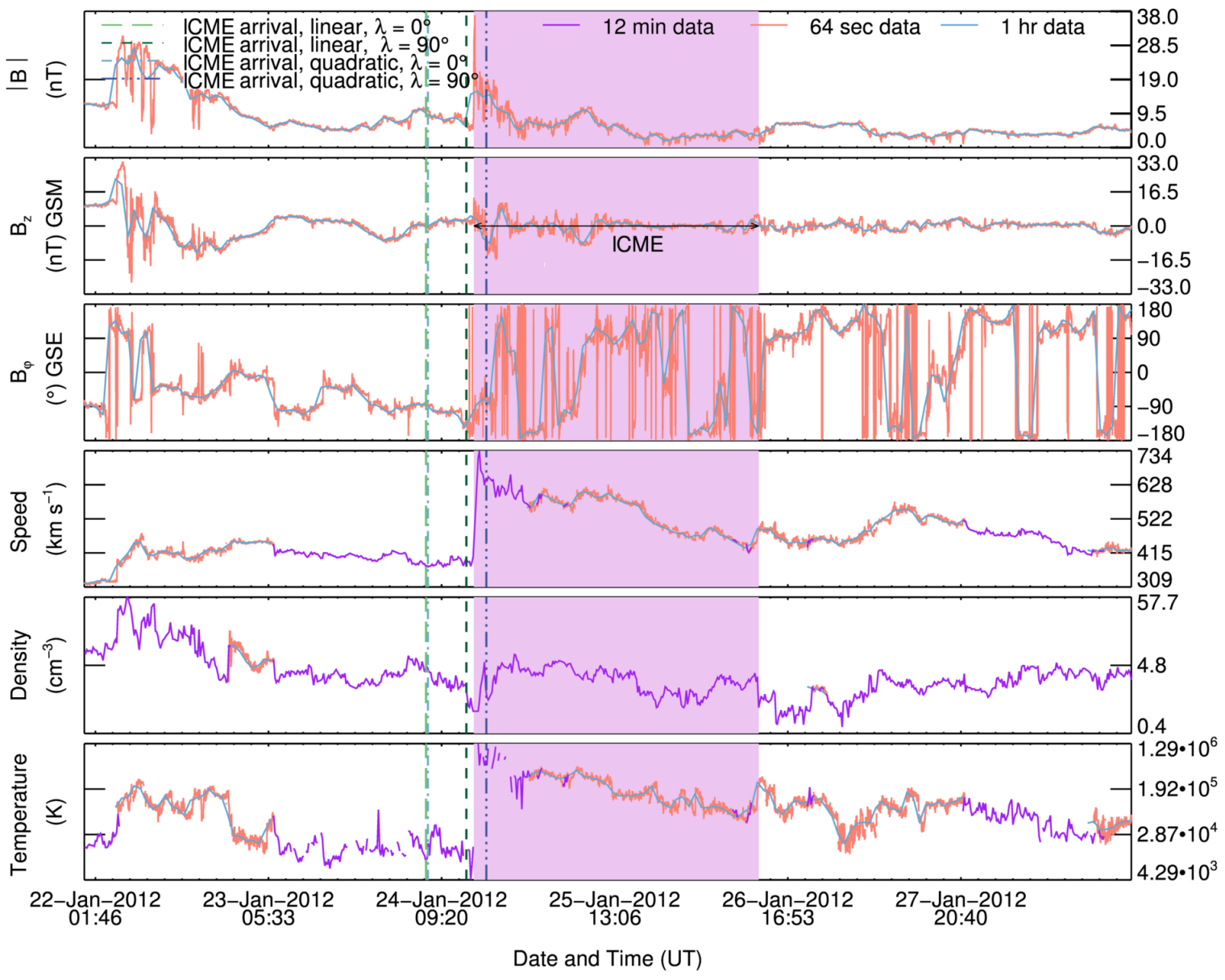

Figure 8.44.: ACE in-situ plasma and magnetic field measurements between 22 and 28 January 2012. The purple area marks the ICME passage. Overplotted are the SSSEM arrival times for $\lambda=0^{\circ}$ and $\lambda=90^{\circ}$ obtained via linear and quadratic fit to the SSSEM height.

\begin{tabular}{llll}
\hline & EUVI+ACE & 1st degree fit $\lambda=0^{\circ}$ & 1st degree fit $\lambda=90^{\circ}$ \\
\hline CME eruption time & 23-Jan-2012 03:38 & 23-Jan-2012 03:34 & 23-Jan-2012 02:17 \\
ICME arrival speed & 730 $\mathrm{km} \mathrm{s}^{-1}$ & 1599 $\mathrm{km} \mathrm{s}^{-1}$ & 1126 $\mathrm{km} \mathrm{s}^{-1}$ \\
ICME arrival time & 24-Jan-2012 14:30 & 24-Jan-2012 06:49 & 24-Jan-2012 13:18 \\
\hline & & & \\
\hline & EUVI+ACE & 2nd degree fit $\lambda=0^{\circ}$ & 2nd degree fit $\lambda=90^{\circ}$ \\
\hline CME eruption time & 23-Jan-2012 03:38 & 23-Jan-2012 03:20 & 23-Jan-2012 00:49 \\
ICME arrival speed & 730 $\mathrm{km} \mathrm{s}^{-1}$ & 1541 $\mathrm{km} \mathrm{s}^{-1}$ & 1009 $\mathrm{km} \mathrm{s}^{-1}$ \\
ICME arrival time & 24-Jan-2012 14:30 & 24-Jan-2012 07:07 & 24-Jan-2012 16:30 \\
\hline
\end{tabular}

Table 8.20.: Observed CME eruption time, arrival time and speed at $L_{1}$ compared to those obtained via a linear (top panel) and quadratic (bottom panel) fit to the CME height-time profile derived by applying the SSSEM for $\lambda=0^{\circ}$ and $\lambda=90^{\circ}$ to STEREO/SECCHI observations of the 23 January 2012 event.

et al. (2013) the CME did not hit the spacecraft at all. They hypothesise that the CME-driven shock, the only in-situ signature of the 23 January 2012 CME, was signif- 
icantly larger than the CME itself. This interpretation is consistent with GCS results for the 3D orientation of the CME. Moreover, looking closely at difference images, it seems rather likely that the feature tracked in the j-maps is indeed the shock signature at the CME flanks, rather than the CME itself.

Table 8.20 shows the arrival times and speed extrapolated to $L_{1}$ compared to those derived from in-situ ACE measurements, as well as eruption times compared to the time of flaring activity. It includes the results derived from a linear and a quadratic fit to the SSSEM heights for $\lambda=0^{\circ}$ and $\lambda=90^{\circ}$. All four cases predict the arrival time with sufficient precision, but the $\lambda=90^{\circ}$ case reaches an accuracy as low as 1 hour and 20 minutes for the constant speed fit. The earlier arrival times for the case $\lambda=0^{\circ}$ are consistent with the SSSEM results, which produce a late fictitious acceleration in the CME propagation. The arrival speeds are higher than the observed ones, with $\lambda=0^{\circ}$ yielding the largest speeds. Including deceleration improves the results for the speed extrapolations, which are nevertheless satisfactory given the accuracy of the employed models.

The 23 January 2012 CME originated from AR 11402, and was associated with an M8.7 flare, EUV waves and post eruptive arcades. GCS modelling was performed for COR2 images, but the extension to larger heliocentric distances proved to be quite challenging due to the large off-Ecliptic component of the CME apex orientation. The GCS speed shows initial increase from $1462 \mathrm{~km} \mathrm{~s}^{-1}$ between 04:08 UT and 05:08 UT to $2632 \mathrm{~km} \mathrm{~s}^{-1}$ between 05:08 UT and 06:49 UT, followed by a decrease to $985 \mathrm{~km} \mathrm{~s}^{-1}$ between 06:49 UT and 10:49 UT. The SSSEM heights are smaller than the ones derived from the GCS, with the ones corresponding to the locations imaged by HI1 exhibiting the higher discrepancies, up to $\simeq 30 \%$. Such behaviour is however expected because the $G C S$ and the SSSEM track very different CME features.

A separate analysis of the CME and the shock kinematics is not possible for this event. The SSSEM results are compatible with propagation towards Earth for both $\lambda=0^{\circ}$ and $\lambda=90^{\circ}$. The speed and heliocentric distance profiles show a similar behaviour up to about $80 R_{\odot}$ for both values of the half-width, with $\lambda=0^{\circ}$ producing larger velocities and heights. Beyond $80 R_{\odot}$ the $\lambda=0^{\circ}$ velocity increases with time. However, no interplanetary forces can explain CME acceleration at such locations, and such increase in the speed is considered an artefact of the model.

ACE plasma and magnetic field measurements show the passage of an interplanetary shock on 24 January 2012 at 14:30 UT with a speed of $730 \mathrm{~km} \mathrm{~s}^{-1}$. No magnetic cloud signatures are observed, possibly because the spacecraft was hit by the shock driven at the CME flanks. The best arrival time predictions correspond to $\lambda=90^{\circ}$ and constant propagations speed. The estimated arrival time is 24 January 2012 at 13:18 UT and the estimated arrival speed is $1126 \mathrm{~km} \mathrm{~s}^{-1}$. 


\subsection{The 07 March 2012 CME}

\subsubsection{Solar source region}

The CME analysed in this section erupted from NOAA AR 11429, located at N17E21 on the solar disk. The magnetogram in Figure 8.45 shows the active region synoptic position and magnetic field configuration. An X5.4 flare was reported by NOAA be-
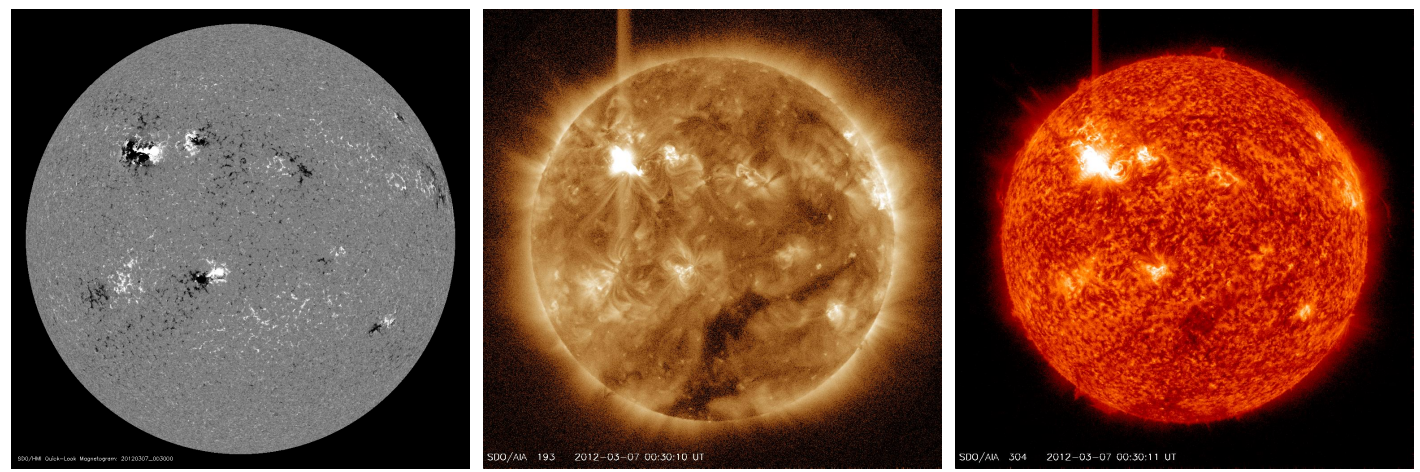

Figure 8.45.: SDO HMI magnetogram, and SDO AIA $193 \AA$ and $304 \AA$ EUV images of the solar disk on 07 March 2012. The brightening in the EUV images corresponds to the flare associated with the CME eruption. Images courtesy of the NASA SDO consortium.

tween 00:02 and 00:40 UT, followed by an X1.3 flare between 01:05 and 01:23 UT. A second CME was probably associated with this flare, but it is not observed as it was hidden by the previous one. A EUV dimming and a wave across the east and north hemispheres were associated with the event. The CME was first seen in COR2 on 07 March 2012 at 01:29 UT. The heliospheric images observed the CME throughout its entire propagation towards Earth, with the first HI1 observations on 07 March 2012 at 01:29 UT, and the first HI2 images on 06:09 UT. Difference images of the event are shown in Figure 8.46. At that time the separation angles between STEREO A and B and Earth were respectively $109^{\circ}$ and $117^{\circ}$.

Shock signatures are visible in COR2 images in form of deflected streamers and as an extended front almost surrounding the flux rope. The complexity of the features observed in the HI1 field of view, however, does not allow a separate study of the CME and the shock evolution.

\subsubsection{GCS modelling}

Since the flux rope is surrounded by an extended bright feature most likely associated with shocked plasma, geometrical modelling for this event is not straightforward. In particular, the extension of the GCS flux rope to HI1 images is hard to interpret, also because of the non negligible angle of propagation of the CME with respect to the Ecliptic plane, which causes a large fraction of the flux rope to propagate outside of the HI1 field of view. However, some of the features seen in COR2 are identifiable in HI1 images as well, indicating a coherent expansion of the CME.

The average speed between 01:08 UT and 02:49 UT was $v=2842 \mathrm{~km} \mathrm{~s}^{-1}$. The 

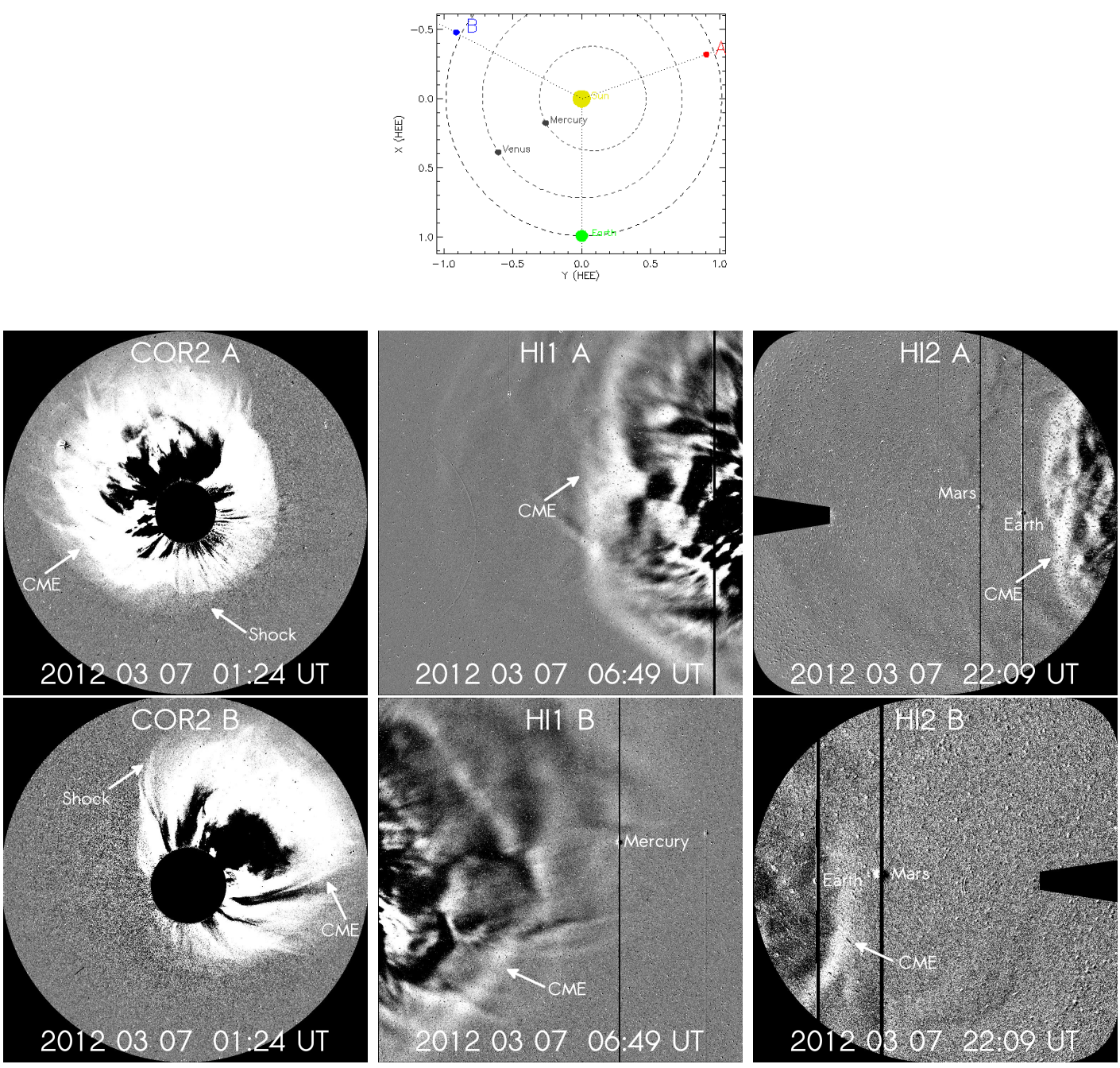

Figure 8.46.: Top panel: positions of the STEREO spacecraft on 07 March 2012. Image credit: NASA STEREO consortium. Bottom panel: difference images obtained from STERE$\mathrm{O} /$ SECCHI A and B COR2 HI1 and HI2 observations, showing subsequent phases of the CME evolution for the 07 March 2012 event.

CME slowed down as it propagated outwards, with an average speed $v=1134 \mathrm{~km} \mathrm{~s}^{-1}$ between 02:40 9U and 06:49 UT. The average acceleration was $-83.52 \mathrm{~m} \mathrm{~s}^{-2}$. The CME radial width in COR2 was $\sim 5 R_{\odot}$, and it increased to about $30 R_{\odot}$ in less than six hours. A comparison with the heights obtained via the SSSEM doesn't seem meaningful for this event, as time-elongation plots on the Ecliptic track features quite distant from the GCS apex.

\subsubsection{SSSEM results and in-situ comparison}

The interplanetary propagation of the CME was studied via the analysis time-elongation plots. The top panel of Figure 8.48 shows the j-maps built from cuts of STEREO A and $\mathrm{B}$ images on the Ecliptic plane. The green dots represent the measured elongations 

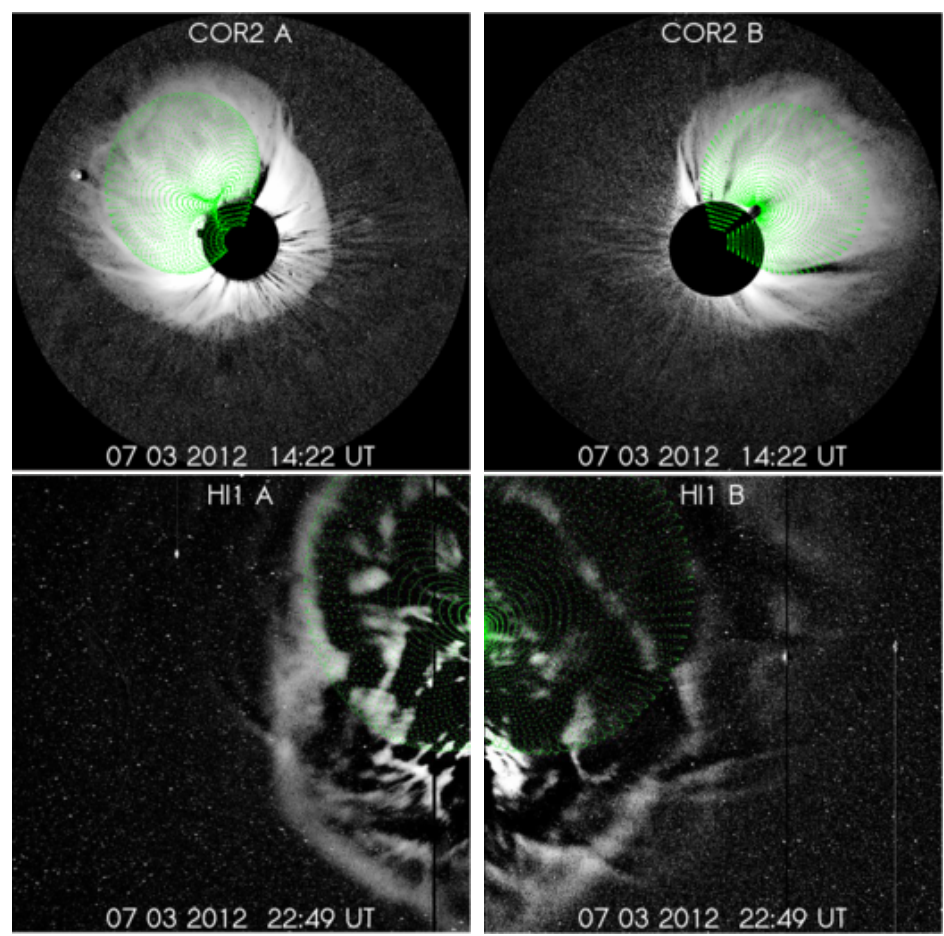

Figure 8.47.: GCS modelling for COR2 (upper panel) and HI1 (lower panels) for observations of the 07 March $2012 \mathrm{CME}$ at 14:22 UT and 22:49 UT.

\begin{tabular}{|r|l|l|l|l|l|l|l|r|}
\hline Instrument & Time & Lon & Lat & Tilt & Height & $\mathrm{k}$ & $\alpha$ & $\mathrm{a}$ \\
\hline COR2 & 07-Mar-2012 01:08:00 & $304.99^{\circ}$ & $36.89^{\circ}$ & $90.00^{\circ}$ & $12.07 R_{\odot}$ & 0.49 & 36.89 & $5.91 R_{\odot}$ \\
HI1 & 07-Mar-2012 02:49:00 & $304.99^{\circ}$ & $36.89^{\circ}$ & $90.00^{\circ}$ & $36.84 R_{\odot}$ & 0.49 & 36.89 & $18.05 R_{\odot}$ \\
HI1 & 07-Mar-2012 06:49:00 & $304.99^{\circ}$ & $36.89^{\circ}$ & $90.00^{\circ}$ & $60.32 R_{\odot}$ & 0.49 & 36.89 & $29.56 R_{\odot}$ \\
\hline
\end{tabular}

Table 8.21.: GCS modelling results for the 07 March 2012 CME. The table shows the imaging instrument, the time of observations, and the GCS parameters: Carrington longitude, heliographic latitude and tilt angle of the solar source region, flux rope height $R_{G C S}$, aspect ratio $k$, half-angle $\alpha$, and radial width $a=k R_{G C S}$.

for both sets of observations. The SSSEM height, speed and direction of propagation with respect to the Sun-Earth line are shown in the bottom panel of Figure 8.48.

The CME heights determined for $\lambda=0^{\circ}$ and $\lambda=90^{\circ}$ are almost the same up to about $50 R_{\odot}$. At larger distances they start diverging, with the $\lambda=0^{\circ}$ case predicting larger CME heights. Correspondingly the $\lambda=0^{\circ}$ speed are higher at larger heliocentric distances than the ones found in the $\lambda=90^{\circ}$ case. Both the velocity profiles show an initial acceleration phase, with peak values of $3000 \mathrm{~km} \mathrm{~s}^{-1}$ for $\lambda=0^{\circ}$ and $2500 \mathrm{~km} \mathrm{~s}^{-1}$ for $\lambda=90^{\circ}$, followed by deceleration. Liu et al. (2013) showed that the speed profile seems to follow quite closely the X-ray flux measured by GOES. In the later stages of propagation the results for $\lambda=90^{\circ}$ predict a roughly constant propagation speed, while for $\lambda=0^{\circ}$ the velocity increases again. This acceleration, similarly to the one found for the 19 January 2012 and 23 January 2012 CMEs, is considered an artefact of the model. The CME direction of propagation with respect to the Sun-Earth line 

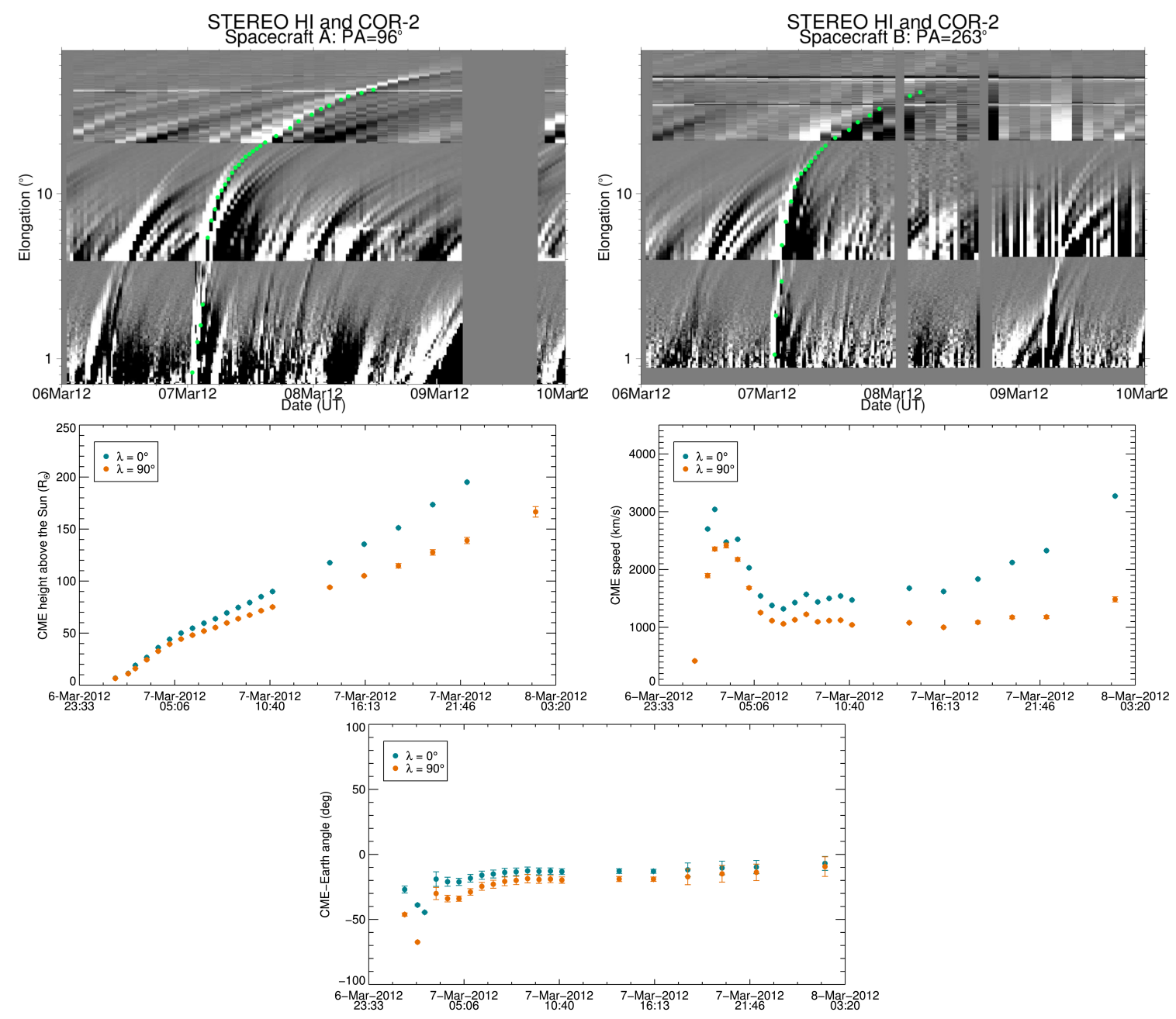

Figure 8.48.: Top panel: j-maps obtained from STEREO A (left) and B (right) observations between 06 and 10 March 2012. The green dots represent the measured elongations used to derive the CME kinematics. Bottom panel: temporal evolution of the CME height, speed and direction of propagation towards Earth obtained applying the SSSEM for $\lambda=0^{\circ}$ and $\lambda=90^{\circ}$ to STEREO/SECCHI observations of the 07 March 2012 event.

shows that the CME moved from an Eastward direction, compatible with the source region longitude (E21) towards smaller angles, i.e. Earthwards on the Ecliptic plane. ACE measurements (see Figure 8.49) show the passage of a shock on 08 March 2012 at 11:15 UT. The purple area in the plot shows the shock sheath and the region in which signatures of the CME passage were identified. The latter crossed ACE on 09 March 2012 at about 02:00 UT. The association between the CME feature tracked in the time-elongation plots and its in-situ counterpart is not obvious for this event. The most likely interpretation is that the feature tracked in the j-maps corresponds to the shock sheath. Difference images,in fact, seem to indicate that the CME is surrounded by shocked material. Moreover the CME apex, according to the GCS model, is highly inclined with respect to the Ecliptic plane. The SSSEM arrival time estimates are therefore compared to the time of shock passage in-situ. 


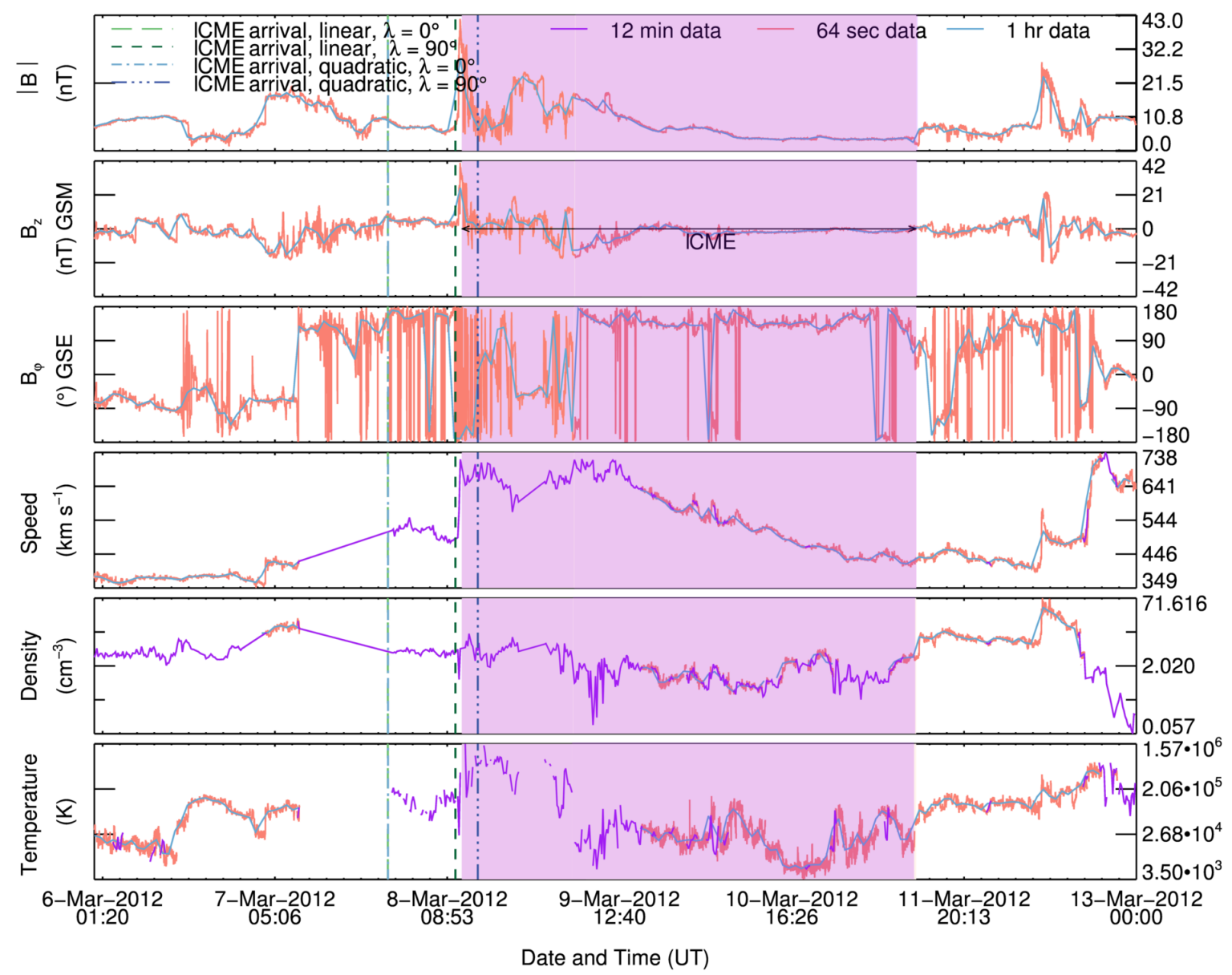

Figure 8.49.: ACE in-situ plasma and magnetic field measurements between 06 and 13 March 2012. The purple area marks the CME passage. Overplotted are the SSSEM arrival times for $\lambda=0^{\circ}$ and $\lambda=90^{\circ}$ obtained via linear and quadratic fit to the SSSEM height.

\begin{tabular}{llll}
\hline & EUVI+ACE & 1st degree fit $\lambda=0^{\circ}$ & 1st degree fit $\lambda=90^{\circ}$ \\
\hline CME eruption time & 07-Mar-2012 00:02 & 07-Mar-2012 01:31 & 06-Mar-2012 22:53 \\
ICME arrival speed & 740 $\mathrm{km} \mathrm{s}^{-1}$ & 1892 $\mathrm{km} \mathrm{s}^{-1}$ & 1167 $\mathrm{km} \mathrm{s}^{-1}$ \\
CME arrival time & 08-Mar-2012 11:15 & 07-Mar-2012 23:19 & 08-Mar-2012 10:12 \\
\hline & & & \\
\hline & EUVI+ACE & 2nd degree fit $\lambda=0^{\circ}$ & 2nd degree fit $\lambda=90^{\circ}$ \\
\hline CME eruption time & 07-Mar-2012 00:02 & 07-Mar-2012 01:23 & 06-Mar-2012 22:45 \\
ICME arrival speed & 740 $\mathrm{km} \mathrm{s}^{-1}$ & 1839 $\mathrm{km} \mathrm{s}^{-1}$ & 1064 $\mathrm{km} \mathrm{s}^{-1}$ \\
ICME arrival time & 08-Mar-2012 11:15 & 07-Mar-2012 23:20 & 08-Mar-2012 13:49 \\
\hline
\end{tabular}

Table 8.22.: Observed CME eruption time, arrival time and speed at $L_{1}$ compared to those obtained via a linear (top panel) and quadratic (bottom panel) fit to the CME height-time profile derived by applying the SSSEM for $\lambda=0^{\circ}$ and $\lambda=90^{\circ}$ to STEREO/SECCHI observations of the 07 March 2012 event.

Arrival time, eruption time and arrival speed are derived assuming constant propagation speed and constant acceleration. The results are summarized in Table 8.22. The 
eruption times are in good agreement with the time of flaring activity. The $\lambda=0^{\circ}$ case always predicts earlier arrival times and higher arrival speeds compared to the $\lambda=90^{\circ}$ case. This is consistent with the SSSEM height and velocity profiles. The best results seem to be associated to $\lambda=90^{\circ}$ and constant speed of propagation.

The inclusion of deceleration does not significantly improve the results for the arrival speeds, which are quite high in comparison to the measured ones. However the arrival time estimates exhibit a satisfactory agreement with observations. Liu et al. (2013) reported that the CME caused a major geomagnetic storm with the Dst index reaching minimum values of $-143 n T$.

The 07 March 2012 CME erupted from AR 11429, and was associated with a X5.4 flare, a EUV dimming and a EUV wave.

Geometrical fitting of COR2 observations was performed via the GCS model, but the extension to the HI1 field of view was not straightforward. Due to the its direction of propagation, in fact, just a small fraction of the CME was imaged by the instrument. The derived average speeds indicate that the CME decelerated during its propagation. Shock signatures are visible in COR2 difference images, but not identifiable in HI1 observations or in time-elongation plots. For this reason the kinematic of the CME and the CME-driven shock could not be be determined separately. The direction of propagation derived via the application of the SSSEM shows a deflection in the trajectory of the CME towards the Sun-Earth line. The speed profiles for both $\lambda=0^{\circ}$ and $\lambda=$ $90^{\circ}$ exhibit an initial increase and a subsequent decrease. After the deceleration phase the speed obtained for $\lambda=90^{\circ}$ remains roughly constant. The case $\lambda=0^{\circ}$ yields propagation at constant speed up to heights around $90 R_{\odot}$, after which the velocity increases again. Such behaviour is however considered only an effect of the model.

ACE in-situ data shows the passage of an interplanetary shock on 08 March 2012 at 11:15 UT, followed by the CME. The shock speed was $740 \mathrm{~km} \mathrm{~s}^{-1}$. The comparison between ACE measurements and the arrival time estimated via a linear and a quadratic fit to the SSSEM heights shows that the best prediction, i.e. arrival on 08 March 2012 at 10:12 UT with a speed of $1167 \mathrm{~km} \mathrm{~s}^{-1}$, is associated to $\lambda=90^{\circ}$ and propagation at constant speed. 


\subsection{The 23 April 2012 CME}

\subsubsection{Solar source region}

The event on 23 April 2012 erupted from NOAA AR 11461 located at N12W20 on the solar disk. The CME was associated with a C2.0 flare between 17:38 and 18:05 UT. SDO observations of the solar disk (see Figure 8.50) show brightness enhancement
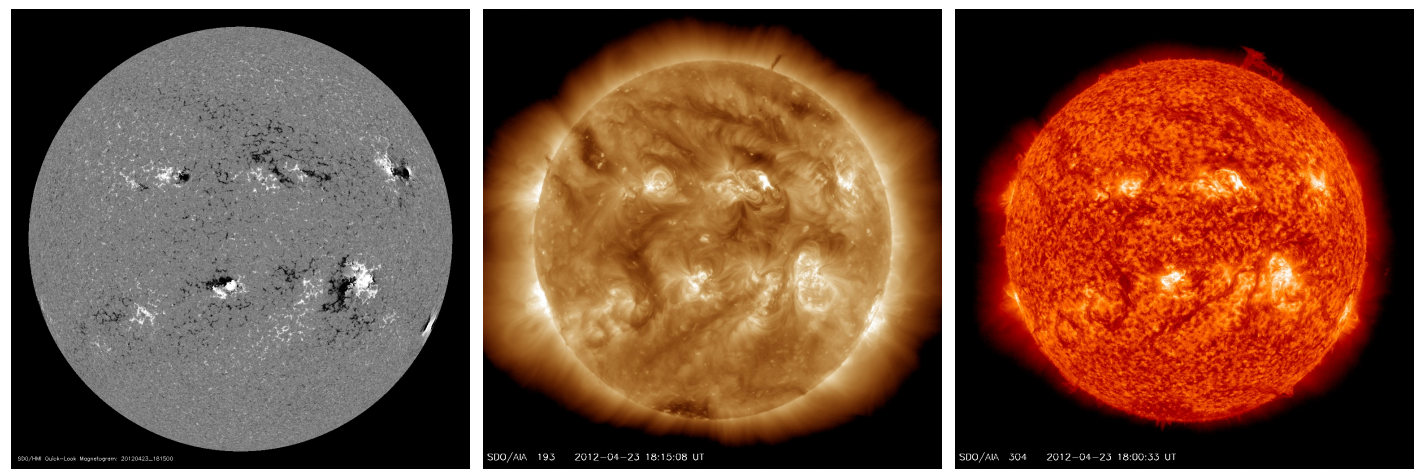

Figure 8.50.: SDO HMI, AIA $193 \AA$ and $304 \AA$ observations of the solar disk on 23 April 2012. Images courtesy of the NASA SDO consortium.

associated with the X-ray flare both in the $304 \AA$ and the $193 \AA$ channels. The underlying active region is visible in the HMI magnetogram. According to the SOHO halo CME alert for this event (http://umbra.nascom.nasa.gov/lasco/observations/ halo/2012/120423/halo-message.20120423) the CME eruption was associated with a southward propagating EUV wave.

At the time of observations the separation angles between Earth and STEREO A and $\mathrm{B}$ were $113^{\circ}$ and $118^{\circ}$ (see Figure 8.51 ) respectively. For this reason, and due to the position of the CME source region, the latter is only marginally visible on the East limb in STEREO EUVI A images, and not visible at all from EUVI B.

The event entered both the COR2 A and B fields of view on 23 April 2012 at 18:24 UT. It was first observed by HI1 A at 20:49 UT on the same day and by HI1 B at 20:09 UT. The event becomes fainter and fainter as it moves towards the outer field of view of the HI1 instruments, and no CME signatures were identified in HI2. In-situ measurements taken from the ACE spacecraft, illustrated in Figure 8.53, also show no indication of the CME passage. Figure 8.51 shows COR2 HI1 and HI2 difference images of the event. The CME morphology deviates from the typical three-part structure with a circular front. For this reason the employment of the GCS model for this event is not meaningful. A bow shock-like feature can be identified in HI1 images, shown in Figure 8.51. Unfortunately, though, this cannot be distinguished from the CME leading edge in time-elongation plots, and a separate analysis of the CME and shock kinematics could not be performed. 

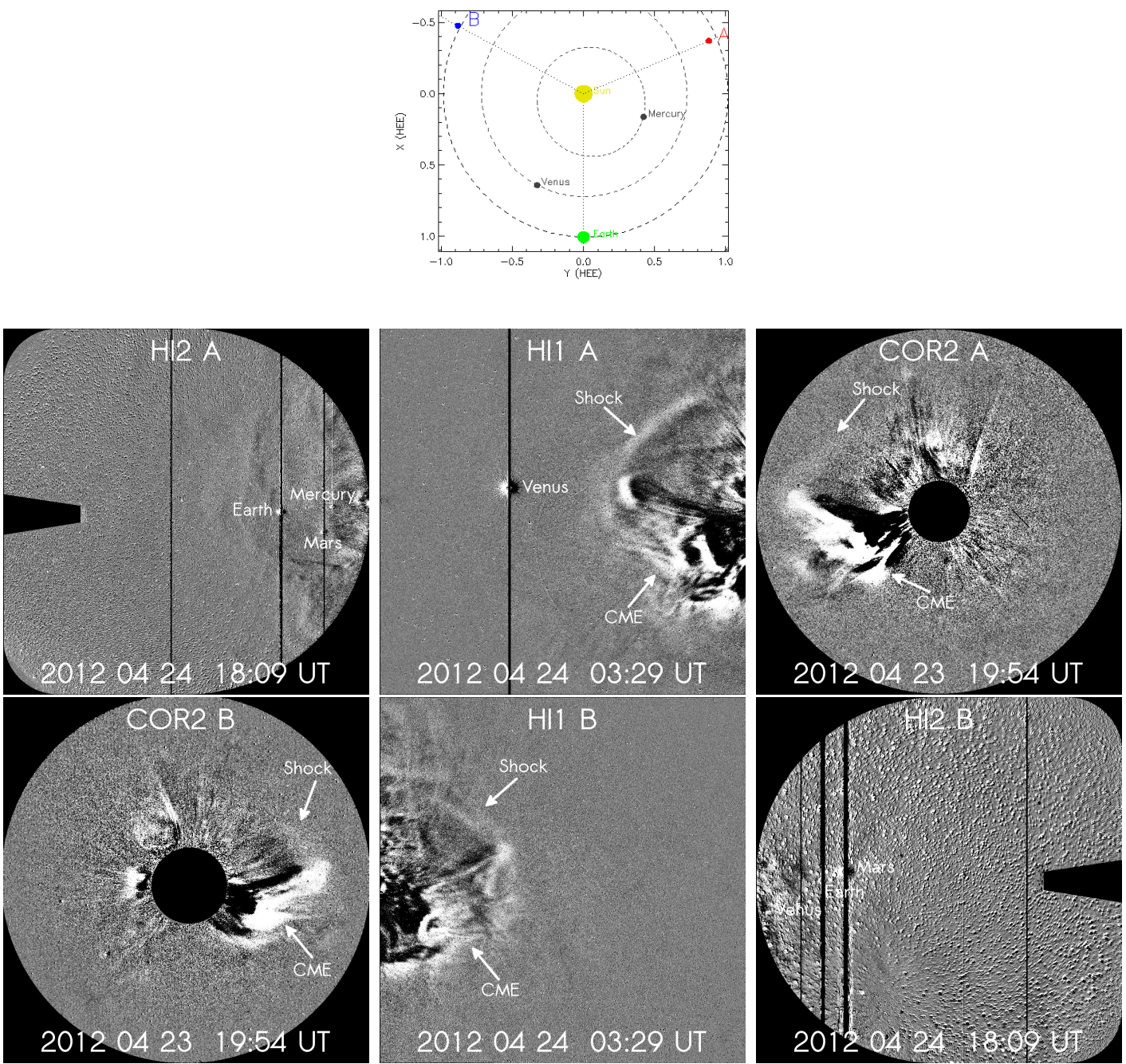

Figure 8.51.: Top panel: positions of the STEREO spacecraft on 24 April 2012. Image credit: NASA STEREO consortium. Bottom panel: difference images obtained from STEREO/SECCHI A (top panel) and B (bottom panel) COR2 HI1 and HI2 observations, showing subsequent phases of the CME evolution for the 24 April 2012 event.

\subsubsection{SSSEM results and in-situ comparison}

The top panel of Figure 8.52 shows the time-elongation plots obtained from STEREO A and B observations between 22 and 27 April 2012. The track corresponding to the event analysed here is the one appearing just before 24 April 2012. The measurement of the time-elongation pairs, indicated with green dots in the j-maps, is restricted to the COR2 and HI1 fields of view, as the CME is not visible in HI2. Applying the SSSEM yields the temporal evolution of the height above the Sun, the speed and the direction of propagation with respect to the Sun-Earth line. These results are shown in the bottom panel of Figure 8.52 for $\lambda=0^{\circ}$ (blue dots) and $\lambda=90^{\circ}$ (red dots). No significant difference is found between the two sets of results.

The direction of propagation seems compatible with propagation towards Earth. An 

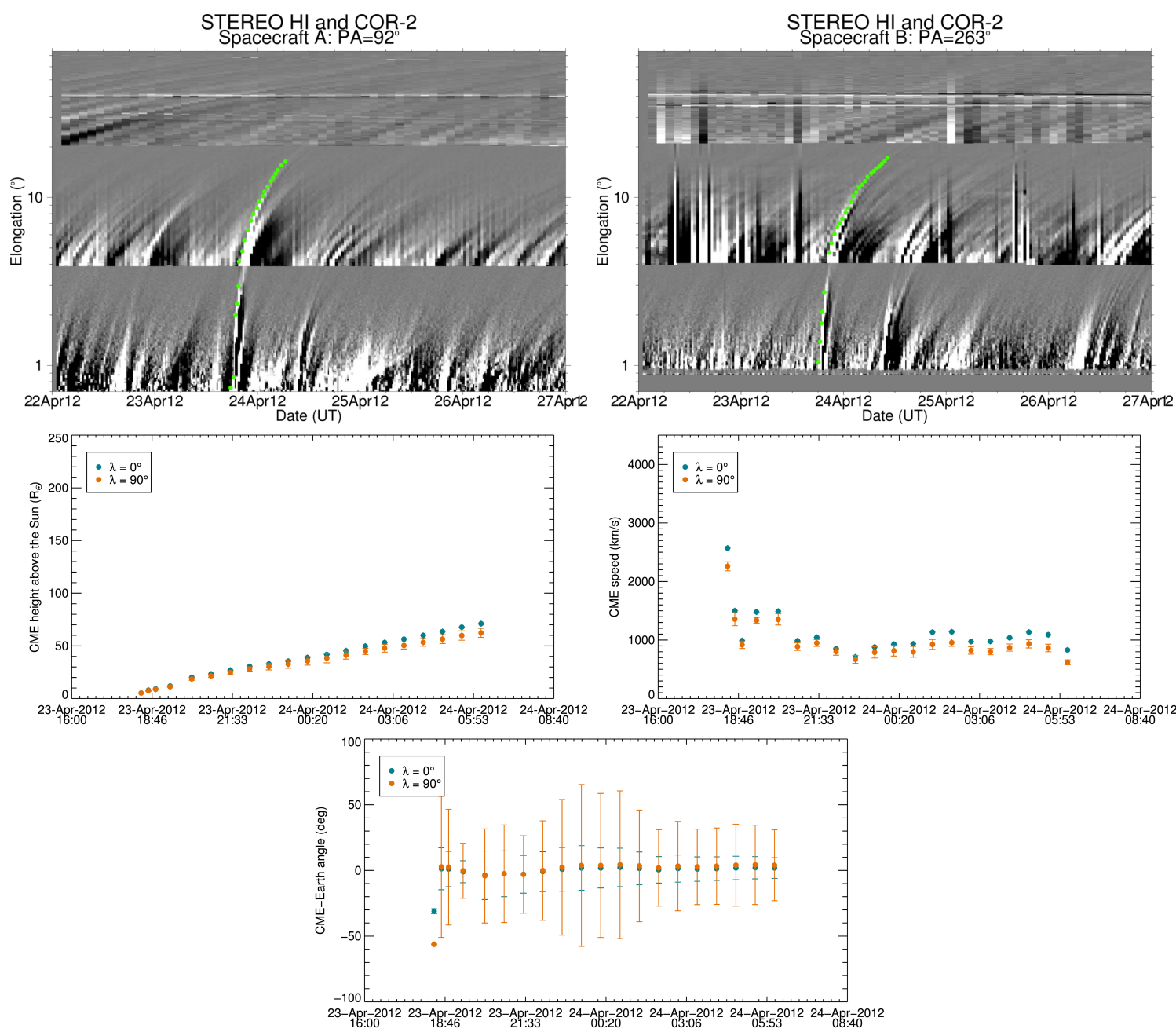

Figure 8.52.: Top panel: j-maps obtained from STEREO A (left) and B (right) observations between 22 and 27 April 2012. The green dots represent the measured elongations used to derive the CME kinematics. Bottom panel: temporal evolution of the CME height, speed and direction of propagation towards Earth obtained by applying the SSSEM for $\lambda=0^{\circ}$ and $\lambda=90^{\circ}$ to STEREO/SECCHI observations of the 23 April 2012 event.

analysis of ACE data though, discussed in the next section, did not reveal any signature of in-situ passage. The speed presents a constant profile at larger distances from the Sun, whereas for COR2 observations an initially accelerating and subsequently decelerating profile is found. Since the CME was not visible in HI2 the maximum value of the CME height is about $80 R_{\odot}$. Figure 8.52 shows the derived CME kinematics for the cases $\lambda=0^{\circ}$ (blue dots) and $\lambda=90^{\circ}$ (red dots) on the Ecliptic plane. The height-time profiles obtained for different values of the half-width $\lambda$ in the HI1 field of view were fitted to a linear and a quadratic function in order to derive arrival times at the location of ACE. The results are summarised in Table 8.23. Eruption times are in good agreement with the $\mathrm{C} 2.0$ flare.

Although the SSSEM direction of propagation is compatible with propagation towards Earth, no signatures of the CME passage are identified in ACE in-situ data. These are 


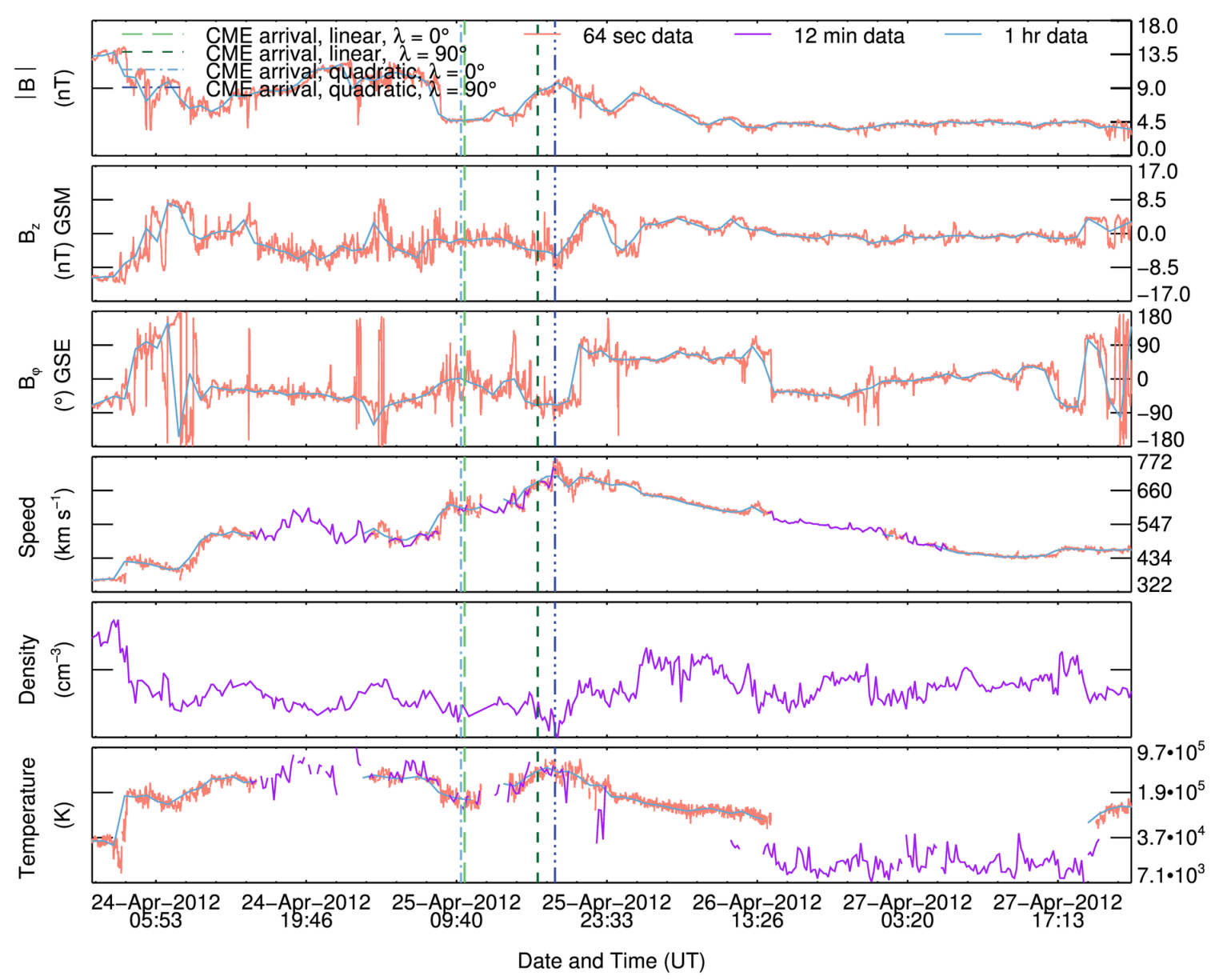

Figure 8.53.: ACE in-situ plasma and magnetic field measurements between 24 and 27 April 2012. No signatures of the passage of an ICME are identified in the data. Overplotted are the SSSEM arrival times for $\lambda=0^{\circ}$ and $\lambda=90^{\circ}$ obtained via linear and quadratic fit to the SSSEM height.

\begin{tabular}{llll}
\hline & EUVI+ACE & 1st degree fit $\lambda=0^{\circ}$ & 1st degree fit $\lambda=90^{\circ}$ \\
\hline CME eruption time & 23-Apr-2012 17:58 & 23-Apr-2012 16:21 & 23-Apr-2012 15:48 \\
CME arrival speed & & $980 \mathrm{~km} / \mathrm{s}$ & 835 km $/ \mathrm{s}$ \\
CME arrival time & & 25-Apr-2012 10:24 & 25-Apr-2012 17:09 \\
\hline & & & \\
\hline & EUVI+ACE & 2nd degree fit $\lambda=0^{\circ}$ & 2nd degree fit $\lambda=90^{\circ}$ \\
\hline CME eruption time & 23-Apr-2012 17:58 & 23-Apr-2012 17:12 & 23-Apr-2012 16:32 \\
ICME arrival speed & & $935 \mathrm{~km} / \mathrm{s}$ & $731 \mathrm{~km} / \mathrm{s}$ \\
ICME arrival time & & 25-Apr-2012 10:04 & 25-Apr-2012 18:43 \\
\hline
\end{tabular}

Table 8.23.: Observed CME eruption time, arrival time and speed at $L_{1}$ compared to those obtained via a linear (top panel) and quadratic (bottom panel) fit to the CME height-time profile derived applying the SSSEM for $\lambda=0^{\circ}$ and $\lambda=90^{\circ}$ for the 23 April 2012 event.

shown in Figure 8.53 for the time period between 24 and 27 April 2012. Overplotted are the inferred arrival times reported in Table 8.23. While one could attribute the 
declining velocity profile between April 25 at about 19:00 UT and April 27 at about 17:30 UT to the passage of the CME across the instrument, this in not associated to any typical CME signature in the density, temperature, or magnetic field profiles. Therefore a connection between remote-sensing observations and in-situ data is not possible for this event.

The event analyzed in this section erupted on 23 April 2012 from AR 11461 and was associated with a C2.0 flare and a EUV wave. The CME was observed by STEREO A and B COR2 and HI1. HI2 images do not show the presence of the CME. Geometrical modeling with the GCS model was not possible for this event, because of its complex morphology. Signatures of a bow shock driven by the CME are present in white-light observations, but are not distinguishable in time-elongation plots. No separate shock and CME analysis could therefore be performed. The SSSEM yields similar results for $\lambda=0^{\circ}$ and $\lambda=90^{\circ}$. The CME speed exhibits a rather regular profile, especially at higher heliocentric distances. The direction of propagation is compatible with a CME impact on Earth. Eruption time, arrival time and speed were determined by assuming both propagation and constant speed and at constant deceleration. The eruption times are in agreement with the time of flaring activity. A comparison with ACE in-situ data was attempted in order to verify the arrival time estimates. However, no signatures of the passage of a CME were found in the plasma and magnetic field measurements. 


\subsection{The 12 July 2012 CME}

\subsubsection{Solar source region}

The 12 July 2012 CME originated from NOAA AR 11520 located at S19E06 on the solar disk. GOES measured an X1.4 flare between 15:37 UT and 17:30 UT, and post eruptive arcades, EUV dimmings and waves were associated with the event. Figure
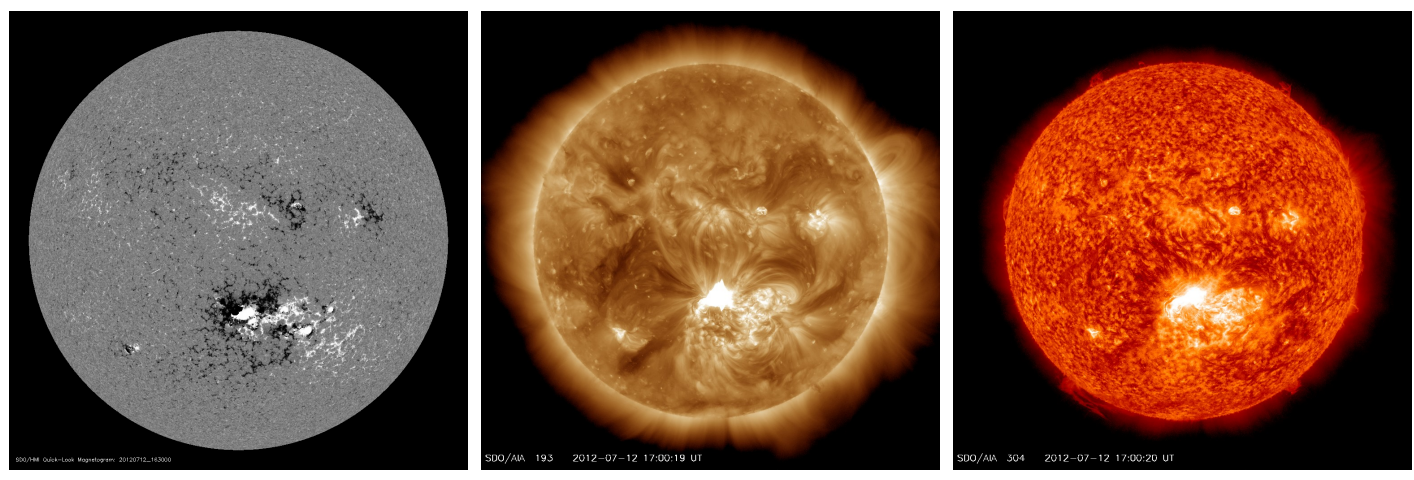

Figure 8.54.: SDO HMI, AIA $193 \AA$ and $304 \AA$ observations of the solar disk on 12 July 2012. Images courtesy of the NASA SDO consortium.

8.54 shows SDO EUV synoptic observations and a magnetogram of the solar disk on the date of the eruption. Both STEREO A and B observed the CME throughout its entire propagation towards Earth. The angular separation from Earth on 12 July 2012 were $120^{\circ}$ for STEREO A and $115^{\circ}$ for STEREO B. The CME source region is therefore seen over the East limb in EUVI A images, and over the West limb in EUVI B images. The CME was first observed in COR2 on 12 July 2012 16:54 UT, and in HI1 and HI2 respectively on 12 July 2012 at 18:49 UT and 13 July 2012 at 10:09 UT. Figure 8.55 illustrates difference images obtained from STEREO A and B observations in different phases of the CME propagation. A faint structure ahead of the CME is visible in both COR2 and HI1 observations, and it corresponds to compressed plasma downstream of the shock. This feature is also visible in time-elongation plots, therefore a full analysis of the CME and shock kinematics as well as of the evolution of the CME-driven shock parameters was possible for this event. The results are illustrated in the remainder of this section.

\subsubsection{GCS modelling}

The CME geometry was fitted with the GCS model using stereoscopic COR2 and HI1 observations, and the results are shown in Table 8.24. The GCS flux rope determined from COR2 observations was extended to the HI1 field of view. While it is possible to define the outer boundary of the flux rope in HI1 images, the GCS model does not seem to be able to accurately represent the observed morphology in the later stages of the CME evolution. The presence of the CME-driven shocks makes the extension more challenging. The model nevertheless provides a good indication on the heights of the flux rope and on its 3D direction of propagation. The average CME speed decreased 

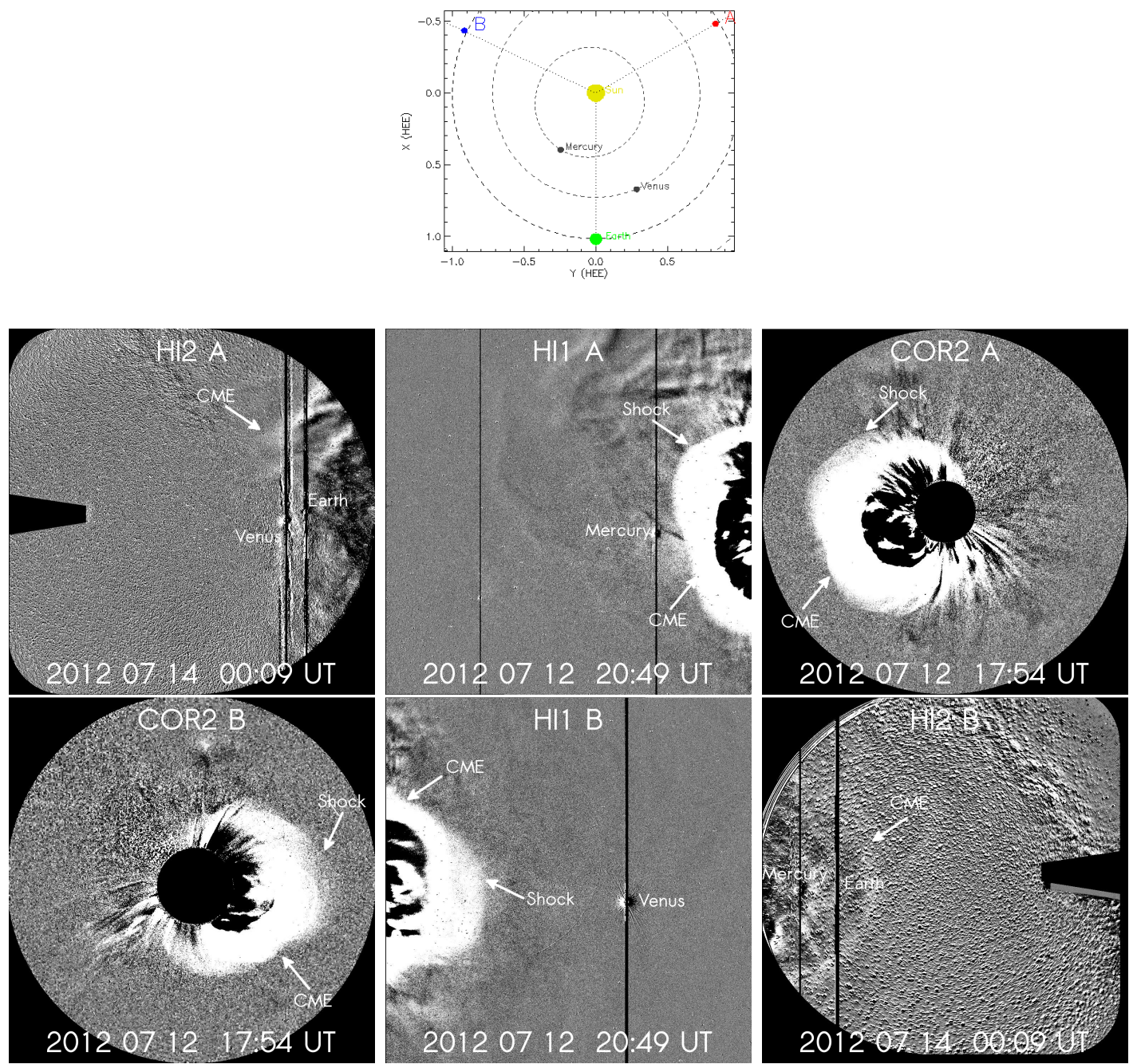

Figure 8.55.: Top panel: positions of the STEREO spacecraft on 12 July 2012. Image credit: NASA STEREO consortium. Bottom panel: difference images obtained from STERE$\mathrm{O} / \mathrm{SECCHI} \mathrm{A}$ and B COR2 HI1 and HI2 observations, showing subsequent phases of the CME evolution for the 12 July 2012 event.

from a value of $1209 \mathrm{~km} \mathrm{~s}^{-1}$ between 18:08 and 22:49 UT on 12 July 2012 to a value of $835 \mathrm{~km} \mathrm{~s}^{-1}$ between 22:49 UT on 12 July 2012 and 04:49 UT on 13 July 2012, corresponding to an average acceleration of $-9.72 \mathrm{~m} \mathrm{~s}^{-2}$. A comparison to the SSSEM results is possible only for the GCS height obtained on 12 July 2012 at 22:49 UT, as no time-elongation measurement is available for the other timestamps. The $\lambda=0^{\circ}$ case yields $52 \pm 1 R_{\odot}$, and the $\lambda=90^{\circ}$ case yields $45 \pm 1 R_{\odot}$. The latter is in good agreement with the GCS height.

\subsubsection{SSSEM results}

The time-elongation plots obtained from cuts of STEREO A and B images along the Ecliptic plane are shown in Figure 8.57. 

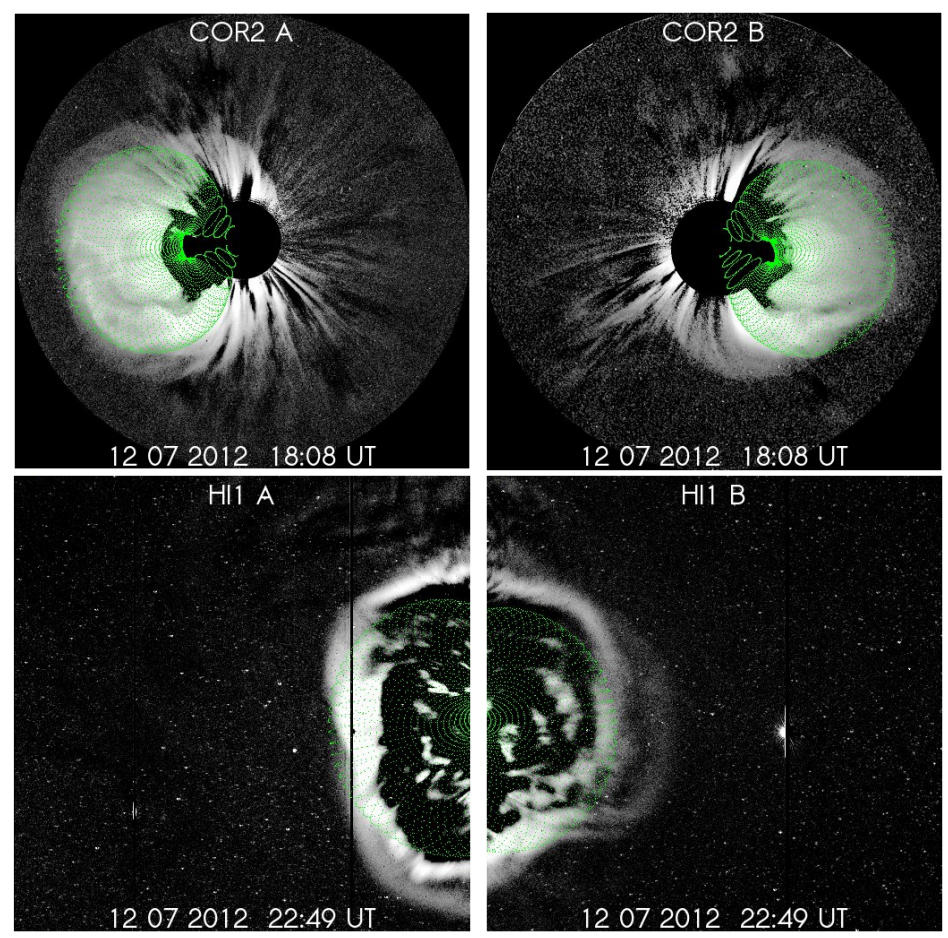

Figure 8.56.: GCS modelling for COR2 (upper panel) and HI1 (lower panels) for observations of the 12 July 2012 CME at 15:08 UT and 18:49 UT.

\begin{tabular}{|r|l|l|l|l|l|l|l|r|}
\hline Instrument & Time & Lon & Lat & Tilt & Height & $\mathrm{k}$ & $\alpha$ & $\mathrm{a}$ \\
\hline COR2 & 12-Jul-2012 18:08:00 & $81.62^{\circ}$ & $-6.71^{\circ}$ & $90.00^{\circ}$ & $13.71 R_{\odot}$ & 0.45 & 37.45 & $6.16 R_{\odot}$ \\
HI1 & 12-Jul-2012 22:49:00 & $81.62^{\circ}$ & $-6.71^{\circ}$ & $90.00^{\circ}$ & $43.03 R_{\odot}$ & 0.45 & 37.45 & $19.36 R_{\odot}$ \\
HI1 & 13-Jul-2012 04:49:00 & $81.62^{\circ}$ & $-6.71^{\circ}$ & $90.00^{\circ}$ & $68.98 R_{\odot}$ & 0.45 & 37.45 & $31.04 R_{\odot}$ \\
\hline
\end{tabular}

Table 8.24.: GCS modelling results for the 12 July 2012 CME. The table shows the imaging instrument, the time of observations, and the GCS parameters: Carrington longitude, heliographic latitude and tilt angle of the solar source region, flux rope height $R_{G C S}$, aspect ratio $k$, half-angle $\alpha$, and radial width $a=k R_{G C S}$.

The green and red dots show the measured time-elongation pairs for the CME and the shock respectively. These were used to determine the kinematics of the two features independently and to infer the values of the CME-driven shock parameters. The results are shown in the next subsections.

\section{CME}

The SSSEM results obtained for $\lambda=0^{\circ}$ and $\lambda=90^{\circ}$ are shown in Figure 8.58. They show the temporal evolution of the CME height, speed, and direction of propagation with respect to the Sun-Earth line. The CME speed profile shows an increase followed by a decrease and finally a more constant behaviour. The direction of propagation is roughly constant, indicative of radial propagation towards Earth. The average angle of propagation with respect to the Sun-Earth line is $\delta_{E}=1^{\circ} \pm 1^{\circ}$ for $\lambda=0^{\circ}$ and 

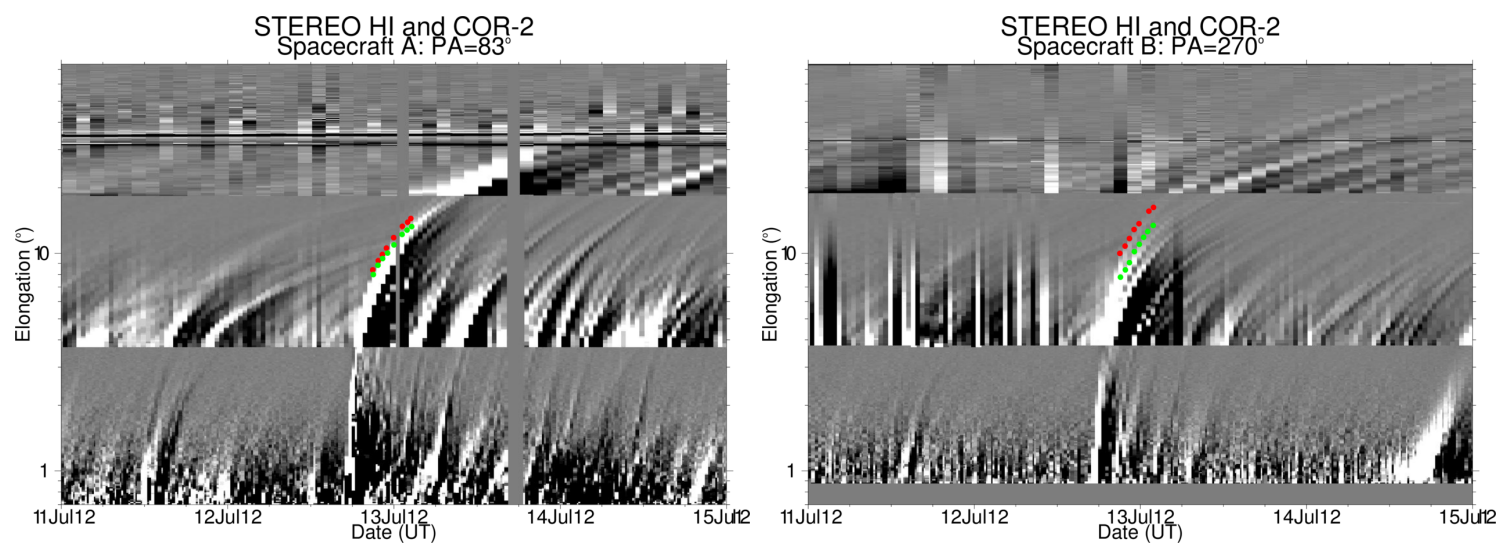

Figure 8.57.: Top panel: j-maps obtained from STEREO A (left) and B (right) observations between 11 and 15 July 2012. The red dots represent the time-elongation measurements for the shock, and the green dots are the time-elongation pairs measured for the CME.
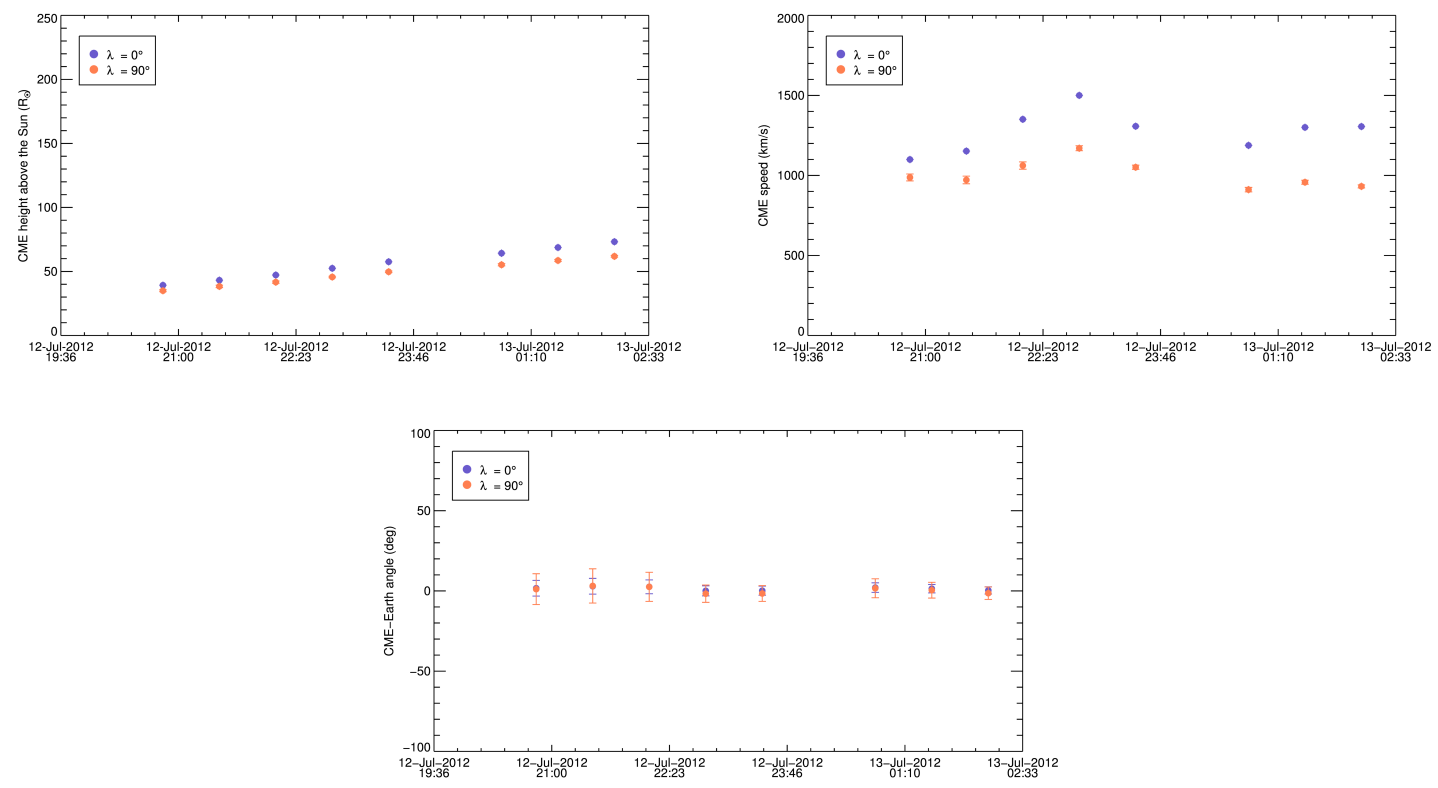

Figure 8.58.: Time evolution of the CME height, speed and direction of propagation towards Earth obtained by applying the SSSEM for $\lambda=0^{\circ}$ and $\lambda=90^{\circ}$ to STEREO/SECCHI observations of the 12 July 2012 event.

$\delta_{E}=1 \pm 2^{\circ}$ for $\lambda=90^{\circ}$, compatible with a CME travelling towards Earth.

\section{Shock}

The shock kinematics was again determined by applying the SSSEM to the timeelongation measurements obtained from the j-maps. The results are shown in Figure 8.59 for $\lambda=0^{\circ}$ and $\lambda=90^{\circ}$. The shock speed shows a slight increase and then a decrease towards a more constant and regular profile for both values of $\lambda$. The direction of propagation is again compatible with radial propagation along the Sun- 

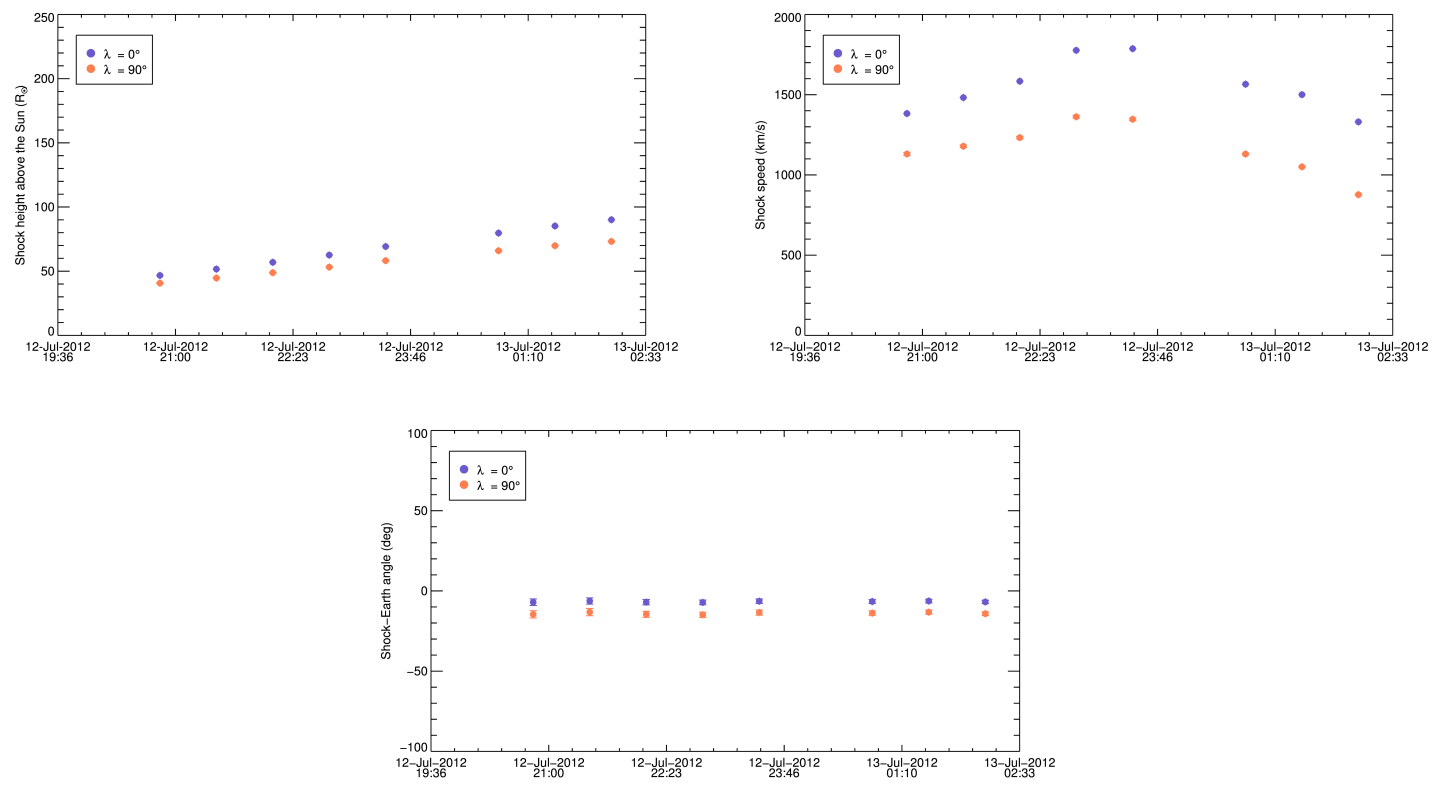

Figure 8.59.: Time evolution of the shock height, speed and direction of propagation towards Earth obtained by applying the SSSEM for $\lambda=0^{\circ}$ and $\lambda=90^{\circ}$ to STEREO/SECCHI observations of the 12 July 2012 event.

Earth line, with average values $\delta_{E}=-7^{\circ} \pm 1^{\circ}$ for both $\lambda=0^{\circ}$ and $\lambda=90^{\circ}$. These results, in combination with those obtained for the CME, allow the determination of the temporal evolution of the shock parameters.

\subsubsection{CME-driven shock parameters}

The standoff distance, the CME radius of curvature, the compression ratio and the Mach number as a function of time were computed using the SSSEM results and the models described in Chapter 7. Their time profiles are shown in Figure 8.60. The case $\lambda=0^{\circ}$ is excluded from these results (see Section 7.0.4). The standoff distance was found to increase with time, and the radius of curvature for $\lambda=90^{\circ}$ is unsurprisingly larger than the one for $\lambda=10^{\circ}$. The Mach number and the compression ratio both show a roughly constant profile, the latter yielding unphysical values $\frac{\rho_{d}}{\rho_{u}}<1$ for $\lambda=10^{\circ}$.

\subsubsection{In-situ comparison}

The time profiles of the CME and shock height above the Sun determined via the application of the SSSEM were fitted to a first and a second degree function in order to derive arrival times and speeds at the location of ACE and compare them to observations. ACE solar wind plasma and magnetic field measurements are shown in Figure 8.61 for the time period between 13 and 17 July 2012. The sudden increase in solar wind speed, temperature, density, and magnetic field corresponds to the passage of the shock across the spacecraft on 14th of July 2012 at 03:49 UT. The CME hit the space- 

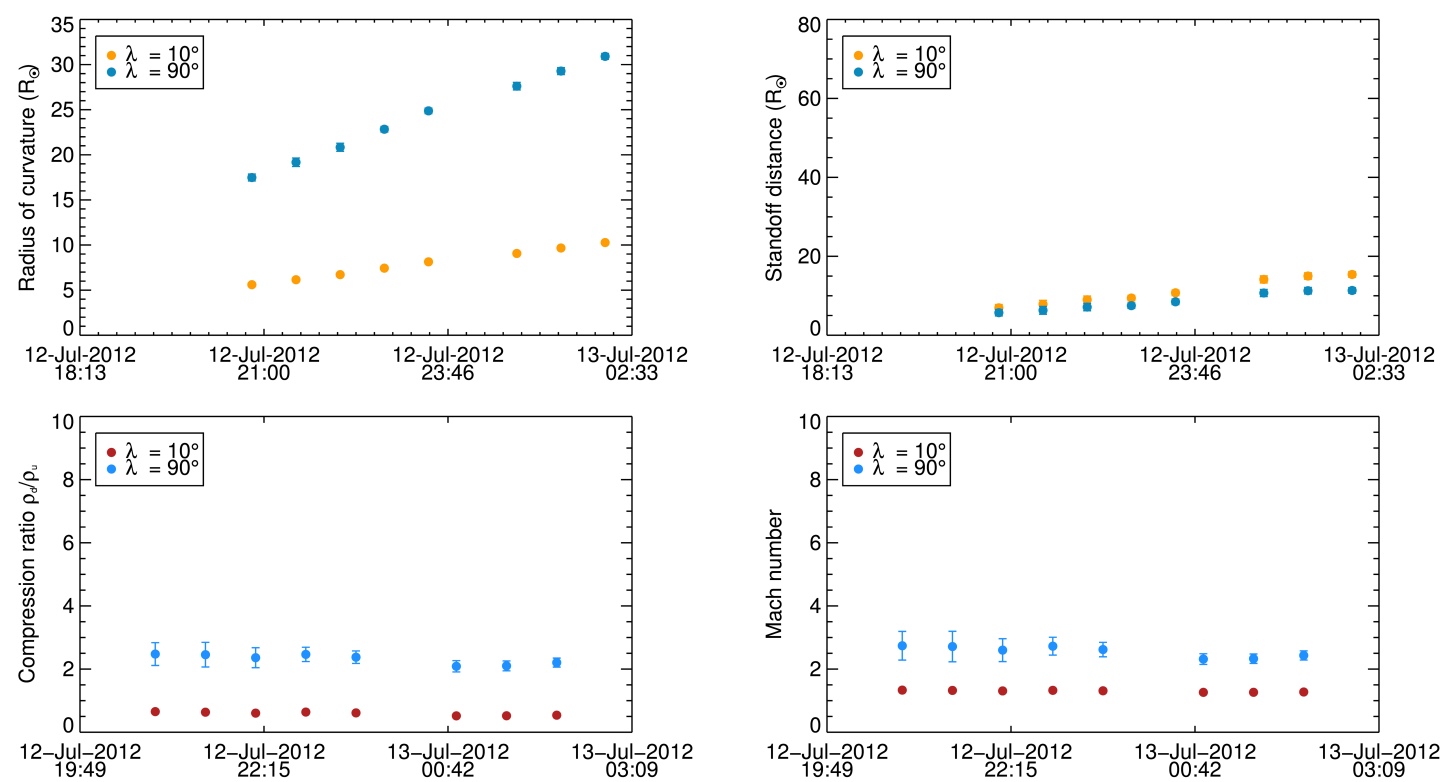

Figure 8.60.: Time evolution of the CME radius of curvature, the shock standoff distance, the compression ratio and the Mach number obtained applying the SSSEM for $\lambda=10^{\circ}$ and $\lambda=90^{\circ}$ to STEREO/SECCHI observations of the 12 July 2012 event.

craft on 14 July 2012 at 11:18 UT. The blue shaded area in Figure 8.61 plot indicates the shock sheath region and the purple shaded area indicates the CME. Overplotted are the arrival times obtained for the CME and the shock for $\lambda=0^{\circ}$ and $\lambda=90^{\circ}$ in the case of propagation at constant deceleration. The full comparison of the arrival times and speed for $\lambda=0^{\circ}$ and $\lambda=90^{\circ}$ is illustrated in Table 8.25. It includes the results for the linear and the quadratic fit as well as the observed eruption time, arrival time for the CME and the shock, and the shock arrival speed. The arrival times determined from the SSSEM are earlier than the observed ones in all the analysed cases, with the

\begin{tabular}{llll}
\hline & EUVI + ACE & 1st degree fit $\lambda=0^{\circ}$ & 1st degree fit $\lambda=90^{\circ}$ \\
\hline CME eruption time & 12-Jul-2012 13:41 & 12-Jul-2012 14:36 & 12-Jul-2012 13:44 \\
Shock arrival time & 14-Jul-2012 17:35 & 13-Jul-2012 16:58 & 14-Jul-2012 02:05 \\
Shock arrival speed & 600 $\mathrm{km} \mathrm{s}^{-1}$ & 1601 $\mathrm{km} \mathrm{s}^{-1}$ & 1145 $\mathrm{km} \mathrm{s}^{-1}$ \\
CME arrival time & 15-Jul-2012 06:00 & 14-Jul-2012 00:02 & 14-Jul-2012 08:07 \\
\hline & & & \\
\hline & EUVI+ACE & 2nd degree fit $\lambda=0^{\circ}$ & 2nd degree fit $\lambda=90^{\circ}$ \\
\hline CME eruption time & 12-Jul-2012 13:41 & 12-Jul-2012 14:38 & 12-Jul-2012 13:43 \\
Shock arrival time & 14-Jul-2012 17:35 & 13-Jul-2012 17:29 & 14-Jul-2012 03:49 \\
Shock arrival speed & 600 $\mathrm{km} \mathrm{s}^{-1}$ & 1522 $\mathrm{km} \mathrm{s}^{-1}$ & 1057 $\mathrm{km} \mathrm{s}^{-1}$ \\
CME arrival time & 15-Jul-2012 06:00 & 14-Jul-2012 01:15 & 14-Jul-2012 11:18 \\
\hline
\end{tabular}

Table 8.25.: Observed CME eruption time, arrival time and speed at $L_{1}$ compared to those obtained via a linear (top panel) and quadratic (bottom panel) fit to the CME height-time profile derived applying the SSSEM for $\lambda=0^{\circ}$ and $\lambda=90^{\circ}$ to STEREO/SECCHI observations of the 12 July 2012 event. 


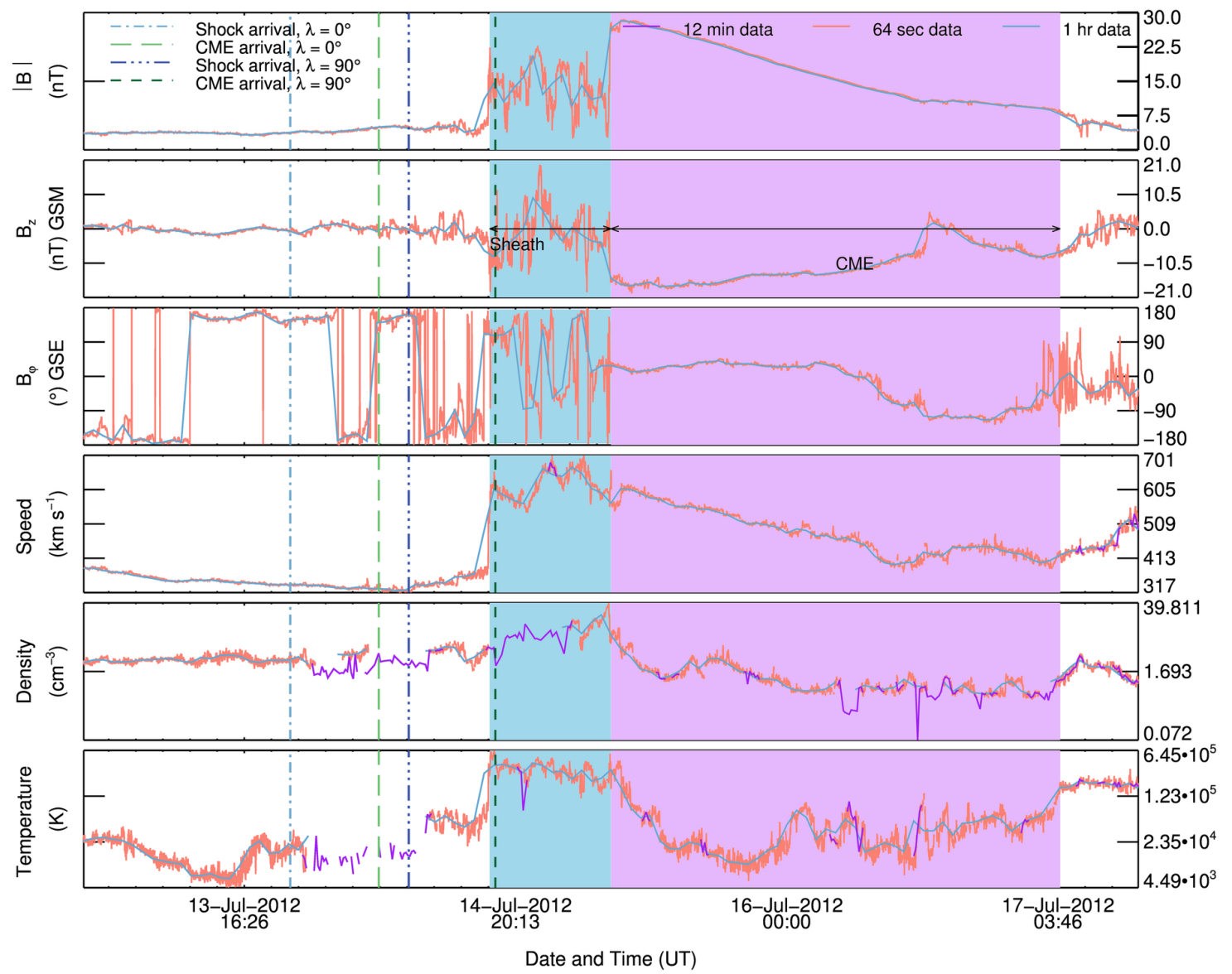

Figure 8.61.: ACE in-situ plasma and magnetic field measurements between 13 and 17 July 2012. The blue area indicates the shock sheath region, and the purple area marks the CME passage. Overplotted are the SSSEM arrival times for $\lambda=0^{\circ}$ and $\lambda=90^{\circ}$ obtained via a quadratic fit to the CME and shock SSSEM heights.

predictions for $\lambda=0^{\circ}$ being almost one day earlier than the actual spacecraft crossing time. The arrival speeds, consequently, are larger than the observed one, with the highest discrepancy being of the order of $1000 \mathrm{~km} \mathrm{~s}^{-1}$. The eruption times are consistent with the observations. The best prediction is found in the case $\lambda=90^{\circ}$ for propagation at constant deceleration, for which the arrival times are between 12 and 14 hours earlier, and the arrival speed is $400 \mathrm{~km} \mathrm{~s}^{-1}$ higher than the in-situ one. The linear and quadratic fit to the CME and shock SSSEM heights were used to determine the in-situ values for the shock parameters. These were compared to the values obtained from ACE observations. Table 8.26 shows the results. The observed standoff distance, compression ratio, and Mach number are $\Delta=38 R_{\odot}, \frac{\rho_{d}}{\rho_{u}}=2.08$, and $M=3.2$. The $\lambda=10^{\circ}$ case, both for the linear and the quadratic fit, results in non-physical values of the compression ratio $\frac{\rho_{d}}{\rho_{u}}<1$. The best fit to observations corresponds to $\lambda=70^{\circ}$, for which $\Delta=30 R_{\odot}, \frac{\rho_{d}}{\rho_{u}}=2.33$, and $M=2.57$ if constant propagation speed is assumed, and to $\lambda=90^{\circ}$ when deceleration is included in the fit to the SSSEM heights, for which $\Delta=32 R_{\odot}, \frac{\rho_{d}}{\rho_{u}}=2.25$, and $M=2.48$. The latter yields the most accurate results. 


\begin{tabular}{llll}
\hline & $\mathrm{ACE}$ & 1st degree fit $\lambda=10^{\circ}$ & 2nd degree fit $\lambda=10^{\circ}$ \\
\hline Standoff distance & $38 R_{\odot}$ & $30 R_{\odot}$ & $31 R_{\odot}$ \\
Compression ratio & 2.08 & 0.73 & 0.72 \\
Mach number & 3.2 & 1.37 & 1.37 \\
\hline & $\mathrm{ACE}$ & 1 st degree fit $\lambda=90^{\circ}$ & 2 nd degree fit $\lambda=90^{\circ}$ \\
\hline Standoff distance & $38 R_{\odot}$ & $30 R_{\odot}$ & $32 R_{\odot}$ \\
Compression ratio & 2.08 & 2.44 & 2.25 \\
Mach number & 3.2 & 2.70 & 2.48 \\
\hline
\end{tabular}

Table 8.26.: Comparison between standoff distance, compression ratio, and Mach number for the 12 July 2012 event as derived from in-situ data, and from the linear and quadratic fits to the SSSEM heights for $\lambda=10^{\circ}$ and $\lambda=90^{\circ}$.

\subsubsection{Corrections for off-Ecliptic propagation}

In this section the discrepancy between the standoff distance and the shock parameter calculated on the Ecliptic plane and at the CME apex is presented (see Table 8.27) for the value of $\lambda$ corresponding to the best fits to observations, i.e. $\lambda=70^{\circ}$ for the linar fit and $\lambda=90^{\circ}$ for the quadratic fit. As can be readily seen, the differences

\begin{tabular}{lll}
\hline Relative error for & 1st degree fit $\lambda=70^{\circ}$ & 2nd degree fit $\lambda=90^{\circ}$ \\
\hline Standoff distance & $0.6 \%$ & $0.6 \%$ \\
CME curvature & $0.6 \%$ & $0.6 \%$ \\
Compression ratio & $0.04 \%$ & $2 \cdot 10^{-5} \%$ \\
Mach number & $0.04 \%$ & $2 \cdot 10^{-5} \%$ \\
\hline
\end{tabular}

Table 8.27.: Relative difference between the standoff distance, the CME radius of curvature, the compression ratio and the Mach number computed on the Ecliptic plane and at the CME apex for the 12 July 2012 event. The results for the value of lambda which yields the best fit to the in-situ shock parameters are shown.

between the values calculated on the Ecliptic and those obtained at the CME apex are negligible. This is consistent with the high-value of lambda and with the fairly small angle of propagation of the CME apex with respect to the Ecliptic plane (i.e. the latitude in Table 8.24). Under these conditions the standoff distance models introduced in Chapter 7 can be reliably used in combination with the SSSEM results.

The 12 July 2012 event erupted from AR 11520. It was associated to an X1.4 flare, post eruptive loops, EUV dimmings and a EUV wave.

GCS modelling was performed by fitting the GCS flux rope to COR2 observations and extending the results to the HI1 field of view. The GCS geometry did not seem to appropriately represent the CME morphology observed in HI1 images. It however pro- 
vides an estimate for the apex height. Agreement with the SSSEM results is only found for $\lambda=90^{\circ}$. The average CME speed according to the GCS results decreased from of $1209 \mathrm{~km} \mathrm{~s}^{-1}$ between 18:08 and 22:49 UT on 12 July 2012 to $835 \mathrm{~km} \mathrm{~s}^{-1}$ between 22:49 UT on 12 July 2012 and 04:49 UT on 13 July 2012.

Signatures of the CME-driven shock were identified in white-light observations and $j$ maps. The CME and shock kinematics were determined separately. For both features the angle of propagation was compatible with propagation along the Sun-Earth line. The speed profiles obtained for the CME and the shock and both values of the SSSEM half-width show an initial increase and then a decrease.

ACE plasma and magnetic field measurements show the passage of an interplanetary shock on 14 July 2012 at 17:35 UT. The shock is followed by the CME, which reached the spacecraft location on 15 July 2012 at 06:00 UT. A comparison between ACE in-situ data and the arrival time and speed prediction obtained via a linear and a quadratic fit to the SSSEM height-time profiles shows that the best fit is found for $\lambda=90^{\circ}$ when the CME and the shock are assumed to propagated constant deceleration. In this case the arrival time discrepancies are between 12 and 14 hours, and the arrival speed is overestimated by $400 \mathrm{~km} \mathrm{~s}^{-1}$.

The derived CME and shock height-time profiles were used to determine the temporal evolution of the shock parameters. The standoff distance was found to increase regularly with time. The Mach number and the density compression show more constant profiles, with the case $\lambda=10^{\circ}$ being associated with non-physical values $\frac{\rho_{d}}{\rho_{u}}<1$. The extrapolation of the shock parameters to $L_{1}$ and the comparison to the values derived from ACE shows that the best prediction is associated with $\lambda=90^{\circ}$ and propagation at constant deceleration. 


\subsection{The 15 March 2013 CME}

\subsubsection{Solar source region}

The event analysed in this section erupted on 15 March 2013 from NOAA AR 11692, located at N11E12 on the solar disk. Figure 8.62 shows SDO EUV images and the magnetogram of the solar disk at the date of CME eruption. SDO AIA observations are not available between 06:24 and 07:39 UT because of on eclipse outage. According to the SOHO halo CME alert message ${ }^{15}$ PROBA 2 images show a flare followed by a EUV dimming, a wave, and post eruptive loops. A long-duration M1.1 flare from AR 11692 is reported by NOAA peaking at 06:38 UT.

STEREO A and B followed the evolution of the CME all the way to Earth. The angular
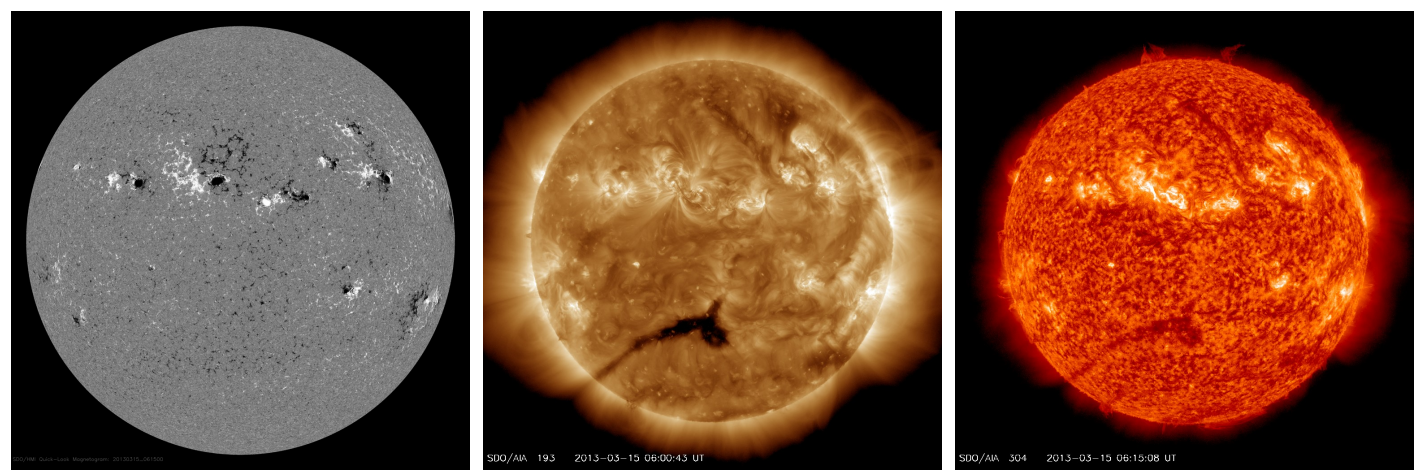

Figure 8.62.: SDO HMI, AIA $193 \AA$ and $304 \AA$ observations of the solar disk on 15 March 2013. Images courtesy of the NASA SDO consortium.

separations between each STEREO A and B and Earth on 15 March 2013 were $131^{\circ}$ and $140^{\circ}$ respectively. The event is seen as an asymmetric halo in COR2 A and B observations, and is back-sided from the STEREO A perspective. Subsequent stages of the CME evolution are presented in Figure 8.63, which shows running difference images obtained from STEREO A and B COR2, HI1 and HI2 observations. Comet PANNStar is visible in HI1 B and HI2B images. The CME drove a shock whose signatures are visible in white-light images and in time-elongation plots. In this case a full analysis of the CME and shock kinematics as well as the determination of the CME-driven shock parameter was possible.

\subsubsection{GCS modelling}

The GCS model was used to fit the CME geometry in COR2 and HI1 images. Table 8.28 shows the derived model parameters. The corresponding GCS flux rope superimposed on STEREO COR2 and HI1 observations is illustrated in Figure 8.64. The GCS model performs fairly well in reproducing the CME geometry as seen in COR2 images. The extension to HI1 observations is not straightforward because of irregularities in the CME morphology. The average CME speed decreased from a value of $1221 \mathrm{~km} \mathrm{~s}^{-1}$

\footnotetext{
${ }^{15}$ http://umbra.nascom.nasa.gov/lasco/observations/halo/2013/130315/
} 

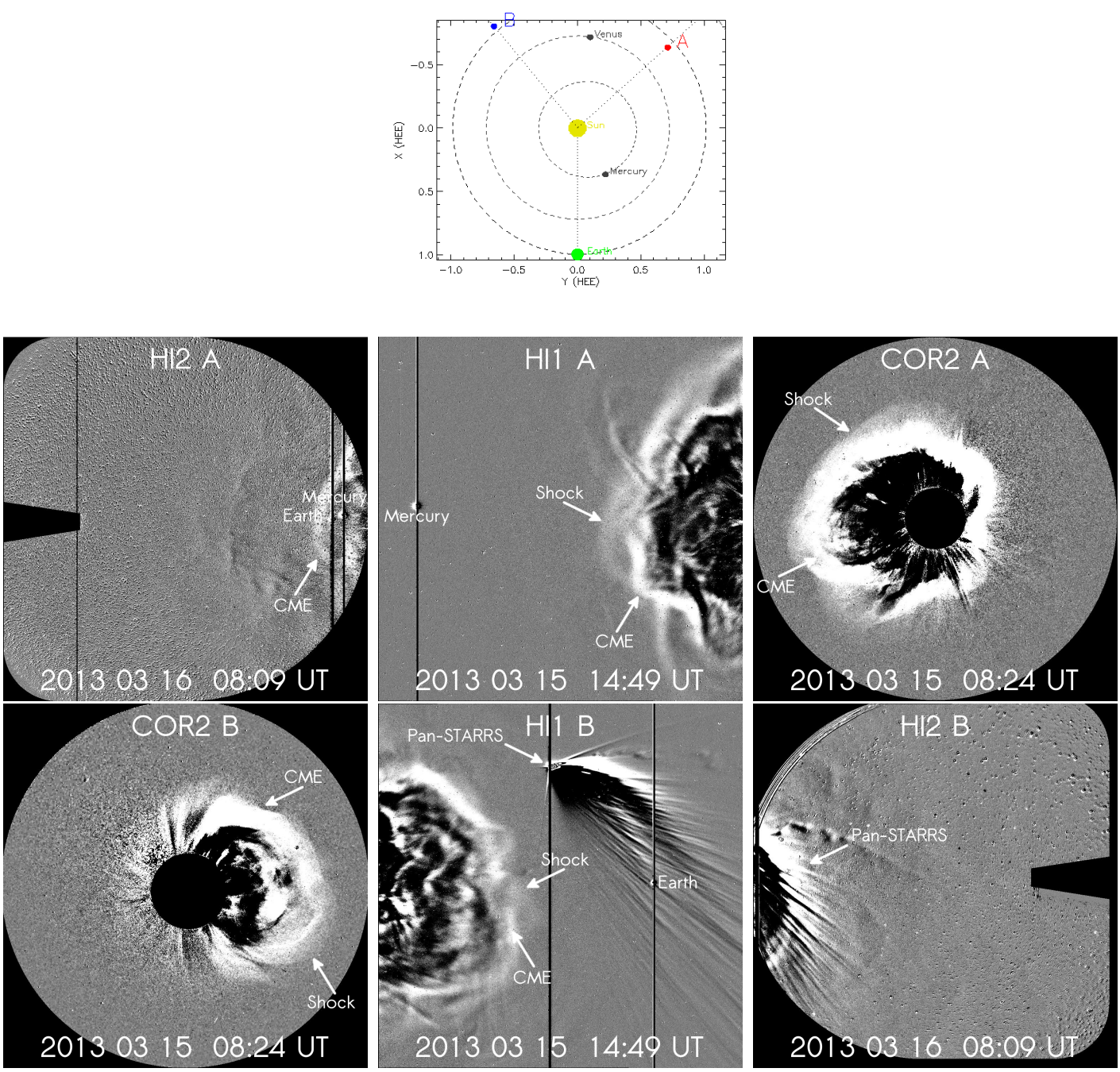

Figure 8.63.: Top panel: positions of the STEREO spacecraft on 15 March 2013. Image credit: NASA STEREO consortium. Bottom panel: difference images obtained from STERE$\mathrm{O} / \mathrm{SECCHI} \mathrm{A}$ and B COR2 HI1 and HI2 observations, showing subsequent phases of the CME evolution for the 15 March 2013 event.

between 08:08 and 14:49 UT to $1044 \mathrm{~km} \mathrm{~s}^{-1}$ between 14:49 and 18:49 UT. The average acceleration was $-4.58 \mathrm{~m} \mathrm{~s}^{-2}$. The SSSEM heights, namely $44 \pm 1 R_{\odot}$ for $\lambda=0^{\circ}$ and $37 \pm 1 R_{\odot}$ for $\lambda=90^{\circ}$ at $14: 49 \mathrm{UT}, 84 \pm 1 R_{\odot}$ for $\lambda=0^{\circ}$ and $57 \pm 1 R_{\odot}$ for $\lambda=90^{\circ}$ at 18:49 UT, do not compare exceptionally well with the GCS heights.

\subsubsection{SSSEM results}

Figure 8.65 shows the time-elongation plots obtained from slices of STEREO A and $\mathrm{B}$ images cut along the Ecliptic plane. The green and red dots indicate the measured time-elongation profiles for the CME and the shock respectively. These were used to separately determine the CME and shock kinematics and to derive the CME-driven shock parameters. 

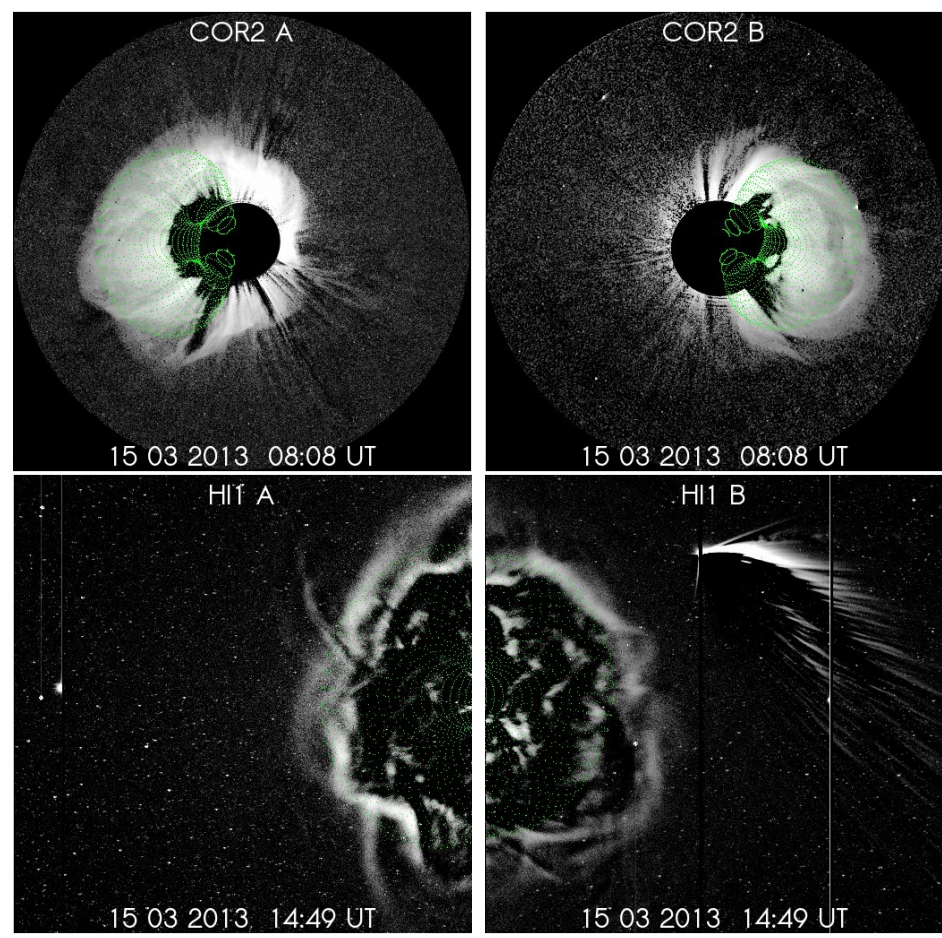

Figure 8.64.: GCS modelling for COR2 (upper panel) and HI1 (lower panels) for observations of the 15 March 2013 CME at 15:08 UT and 18:49 UT. Comet PANNStar is visible in HI1B images.

\begin{tabular}{|r|l|l|l|l|l|l|l|r|}
\hline Instrument & Time & Lon & Lat & Tilt & Height & $\mathrm{k}$ & $\alpha$ & $\mathrm{a}$ \\
\hline COR2 & 15-Mar-2013 08:08:00 & $73.78^{\circ}$ & $-5.03^{\circ}$ & $-83.29^{\circ}$ & $13.14 R_{\odot}$ & 0.36 & 36.16 & $4.73 R_{\odot}$ \\
HI1 & 15-Mar-2013 14:49:00 & $73.78^{\circ}$ & $-5.03^{\circ}$ & $-83.29^{\circ}$ & $55.38 R_{\odot}$ & 0.36 & 36.16 & $19.93 R_{\odot}$ \\
HI1 & 15-Mar-2013 18:49:00 & $73.78^{\circ}$ & $-5.03^{\circ}$ & $-83.29^{\circ}$ & $77.01 R_{\odot}$ & 0.36 & 36.16 & $27.72 R_{\odot}$ \\
\hline
\end{tabular}

Table 8.28.: GCS modelling results for the 15 March 2013 CME. The table shows the imaging instrument, the time of observations, and the GCS parameters: Carrington longitude, heliographic latitude and tilt angle of the solar source region, flux rope height $R_{G C S}$, aspect ratio $k$, half-angle $\alpha$, and radial width $a=k R_{G C S}$.

\section{CME}

The SSSEM results for the temporal evolution of the CME heliocentric distance, speed, and angle of propagation with respect to the Sun-Earth line are shown in Figure 8.66 for $\lambda=0^{\circ}$ (purple dots) and $\lambda=90^{\circ}$ (orange dots). The case $\lambda=0^{\circ}$ yields higher values for both the height and speed. The CME speed seems to exhibit an increase followed by a decrease towards roughly constant values. The average angle of propagation with respect to the Sun-Earth line, $\delta_{E}=11^{\circ} \pm 8^{\circ}$ for $\lambda=0^{\circ}$, and $\delta_{E}=29^{\circ} \pm 10^{\circ}$ for $\lambda=90^{\circ}$, is compatible with the picture of a CME propagating towards Earth. 

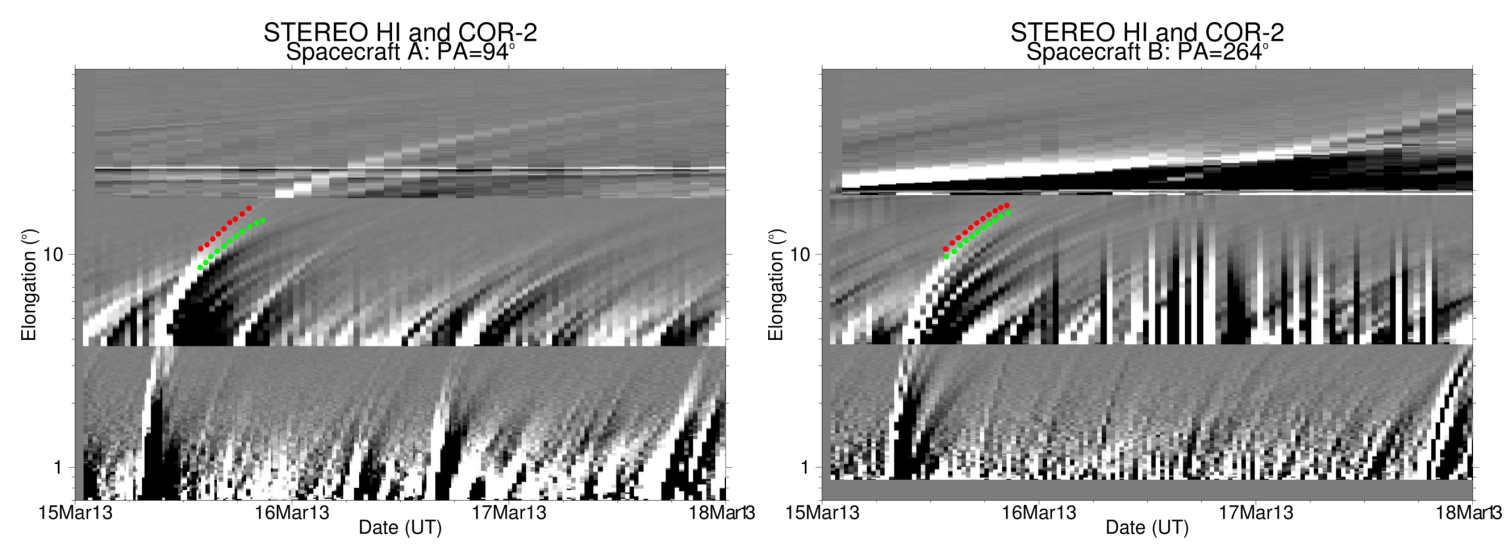

Figure 8.65.: Top panel: j-maps obtained from STEREO A (left) and B (right) observations between 15 and 18 March 2013. The red dots represent the time-elongation measurements for the shock, and the green dots are the time-elongation pairs measured for the CME.
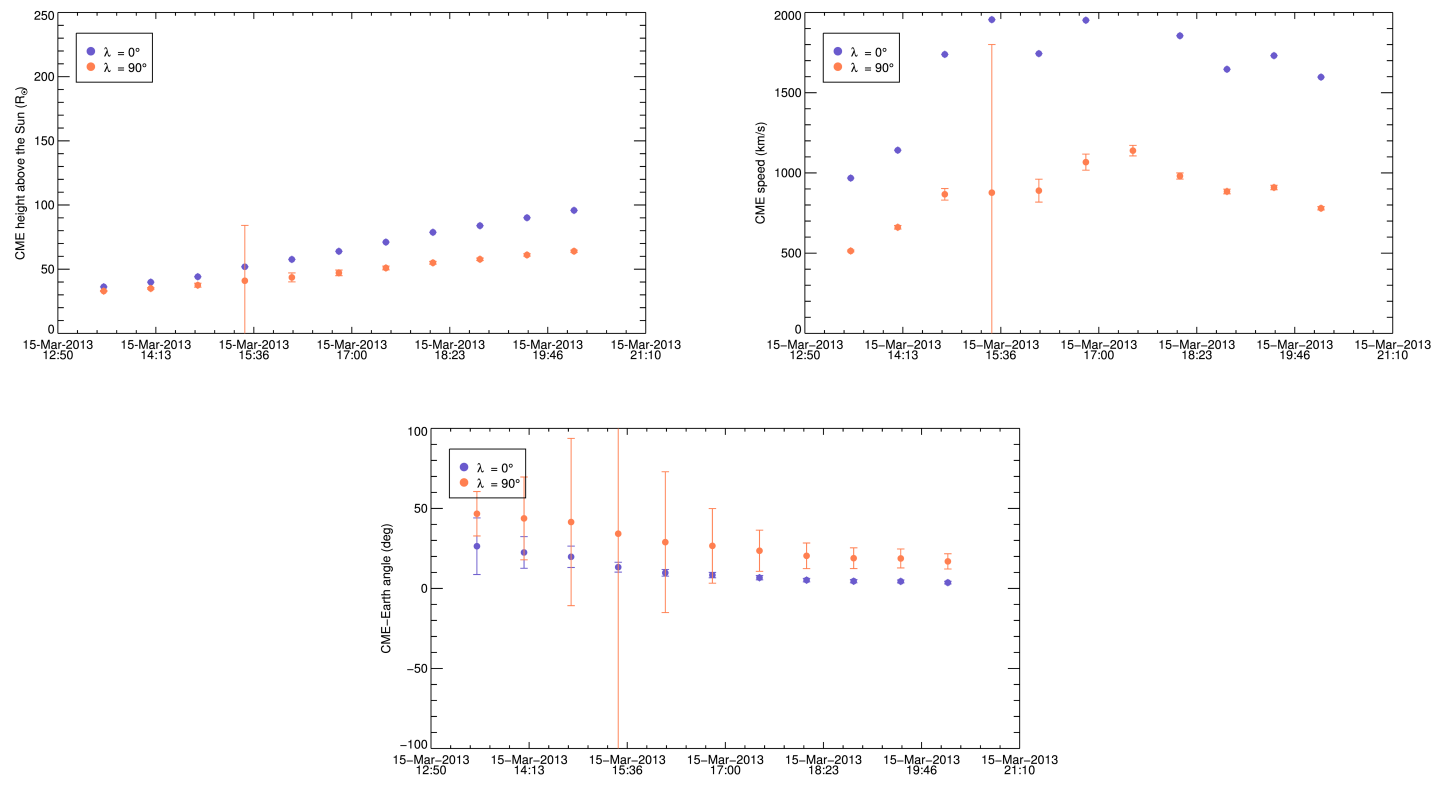

Figure 8.66.: Time evolution of the CME height, speed and direction of propagation towards Earth obtained by applying the SSSEM for $\lambda=0^{\circ}$ and $\lambda=90^{\circ}$ to STEREO/SECCHI observations of the 15 March 2013 event.

\section{Shock}

The time profile of the shock height, speed, and direction of propagation with respect to the Sun-Earth line are shown in Figure 8.67. Similarly to what was found for the CME, the shock height and speed associated to $\lambda=0^{\circ}$ are higher than those corresponding to $\lambda=90^{\circ}$. The average direction of propagation was found to be $\delta_{E}=-8^{\circ} \pm 1^{\circ}$ for $\lambda=0^{\circ}$, and $\delta_{E}=-15^{\circ} \pm 2^{\circ}$ for $\lambda=90^{\circ}$, both consistent with earthward propagation. 

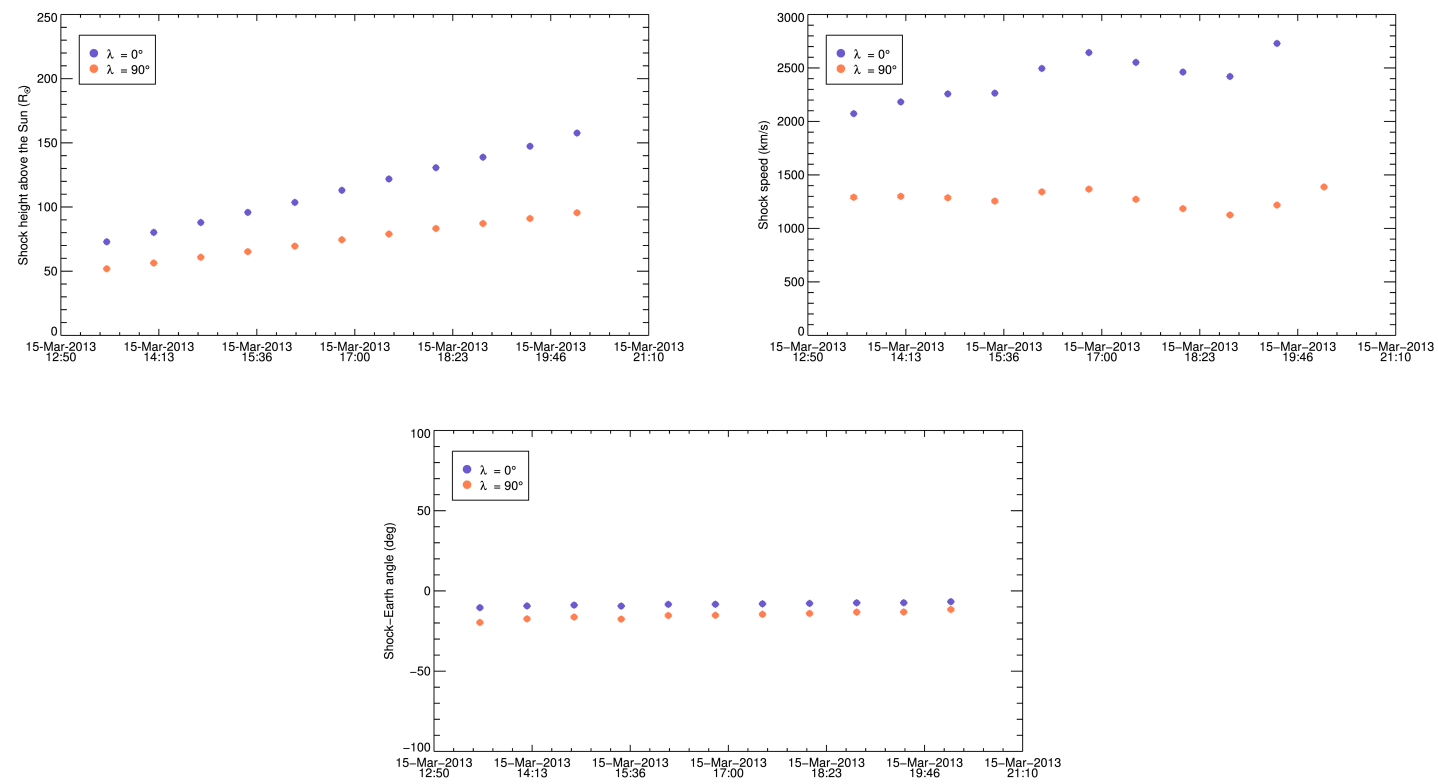

Figure 8.67.: Time evolution of the shock height, speed and direction of propagation towards Earth obtained by applying the SSSEM for $\lambda=0^{\circ}$ and $\lambda=90^{\circ}$ to STEREO/SECCHI observations of the 15 March 2013 event.

\subsubsection{CME-driven shock parameters}
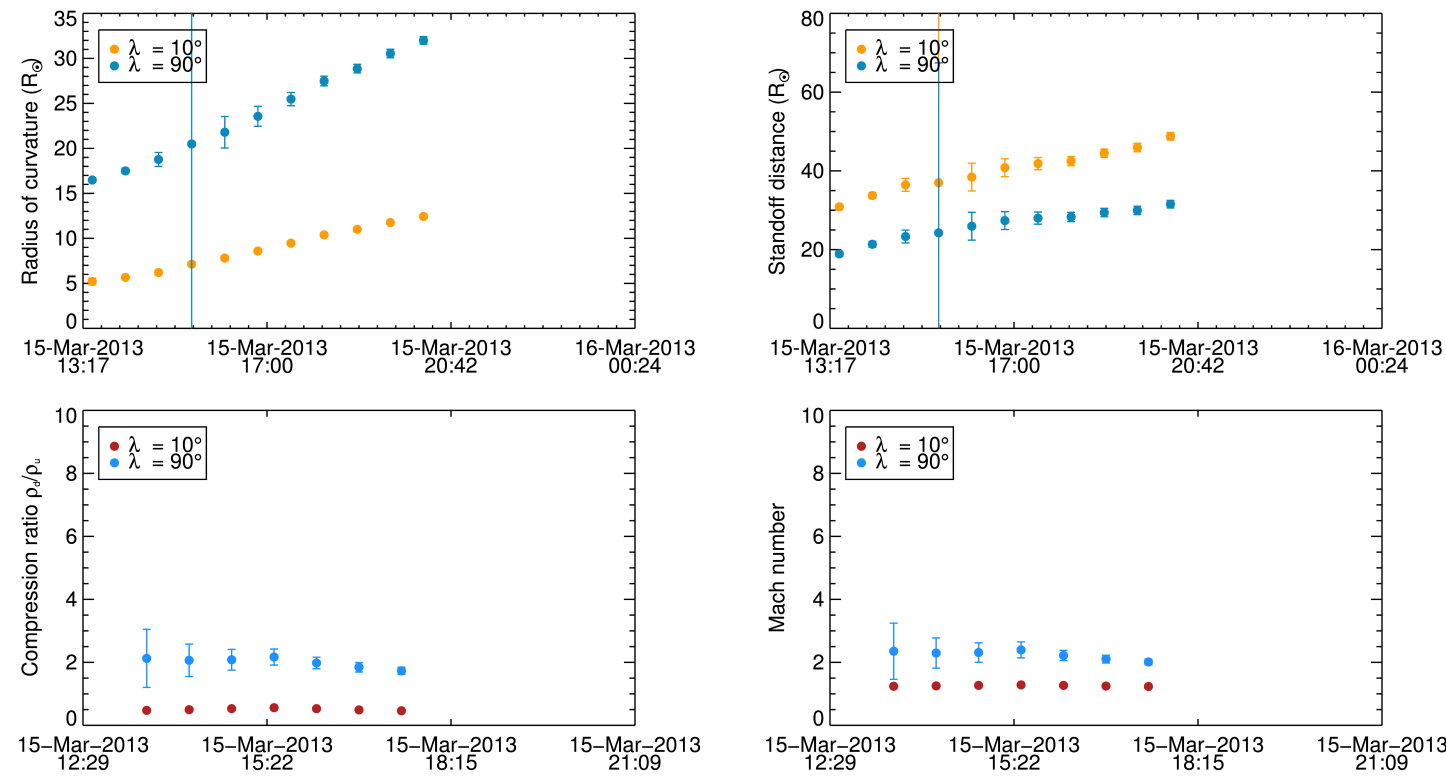

Figure 8.68.: Time evolution of the CME radius of curvature, the shock standoff distance, the compression ratio and the Mach number obtained by applying the SSSEM for $\lambda=10^{\circ}$ and $\lambda=90^{\circ}$ to STEREO/SECCHI observations of the 15 March 2013 event.

Figure 8.68 shows the temporal evolution of the shock parameters obtained by com- 
bining the SSSEM results with the models for the shock sheath thickness introduced in Chapter 7 . The standoff distance and the radius of curvature show a steady increase with time, whereas, similarly to the other events investigated in the study, the Mach number and the compression ratio show rather constant time profiles. The compression ratio for $\lambda=10^{\circ}$ yields $\frac{\rho_{d}}{\rho_{u}}<1$ at all times.

\subsubsection{In-situ comparison}

The SSSEM heights derived for the CME and the shock were fitted to a linear and a quadratic function, and their eruption time, arrival time and speed at $L_{1}$ were derived. The results were compared to ACE plasma and magnetic field measurements (see Figure 8.69). A sudden increase in the plasma parameters marks the passage of the shock across the spacecraft on 17 March 2013 at 05:30 UT. The CME crossed the spacecraft on the same date at 12:30 UT. The blue and purple shaded areas in Figure 8.69 show respectively the shock sheath and the CME as measured from ACE. Table 8.29 shows a comparison between the observed eruption time, arrival time and speed and their values derived via a linear and a quadratic fit to the CME and shock SSSEM heights. The comparison shows that for propagation at constant velocity the arrival speed predictions at $L_{1}$ are significantly larger than the observed ones, and the arrival times are earlier, in some cases by almost one day. If deceleration is included in the fit, on the other hand, the discrepancies are highly reduced. In the case $\lambda=90^{\circ}$ the differences between the observed and extrapolated arrival times are as low as two hours, and even the shock arrival speed is in excellent agreement with observations.

The extrapolation of the CME and shock SSSEM heights to $L_{1}$ allows the determination of the shock parameters at the same location and a comparison with ACE solar wind data. Table 8.30 shows the standoff distance, the compression ratio, and the Mach

\begin{tabular}{llll}
\hline & EUVI+ACE & 1st degree fit $\lambda=0^{\circ}$ & 1st degree fit $\lambda=90^{\circ}$ \\
\hline CME eruption time & 15-Mar-2013 05:46 & 15-Mar-2013 10:02 & 15-Mar-2013 07:29 \\
Shock arrival time & 17-Mar-2013 05:30 & 16-Mar-2013 00:40 & 16-Mar-2013 15:22 \\
Shock arrival speed & 700 $\mathrm{km} \mathrm{s}^{-1}$ & 2456 $\mathrm{km} \mathrm{s}^{-1}$ & 1257 $\mathrm{km} \mathrm{s}^{-1}$ \\
CME arrival time & 17-Mar-2013 12:30 & 16-Mar-2013 08:19 & 17-Mar-2013 07:22 \\
\hline & & & \\
\hline & EUVI+ACE & 2nd degree fit $\lambda=0^{\circ}$ & 2nd degree fit $\lambda=90^{\circ}$ \\
\hline CME eruption time & 15-Mar-2013 05:46 & 15-Mar-2013 09:44 & 15-Mar-2013 05:54 \\
Shock arrival time & 17-Mar-2013 05:30 & 16-Mar-2013 08:04 & 17-Mar-2013 03:52 \\
Shock arrival speed & $700 \mathrm{~km} \mathrm{~s}^{-1}$ & 1879 $\mathrm{km} \mathrm{s}^{-1}$ & 750 km s $\mathrm{s}^{-1}$ \\
CME arrival time & 17-Mar-2013 12:30 & 16-Mar-2013 09:12 & 17-Mar-2013 14:15 \\
\hline
\end{tabular}

Table 8.29.: Observed CME eruption time, arrival time and speed at $L_{1}$ compared to those obtained via a linear (top panel) and quadratic (bottom panel) fit to the CME height-time profile derived by applying the SSSEM for $\lambda=0^{\circ}$ and $\lambda=90^{\circ}$ to STEREO/SECCHI observations of the 15 March 2013 event. 


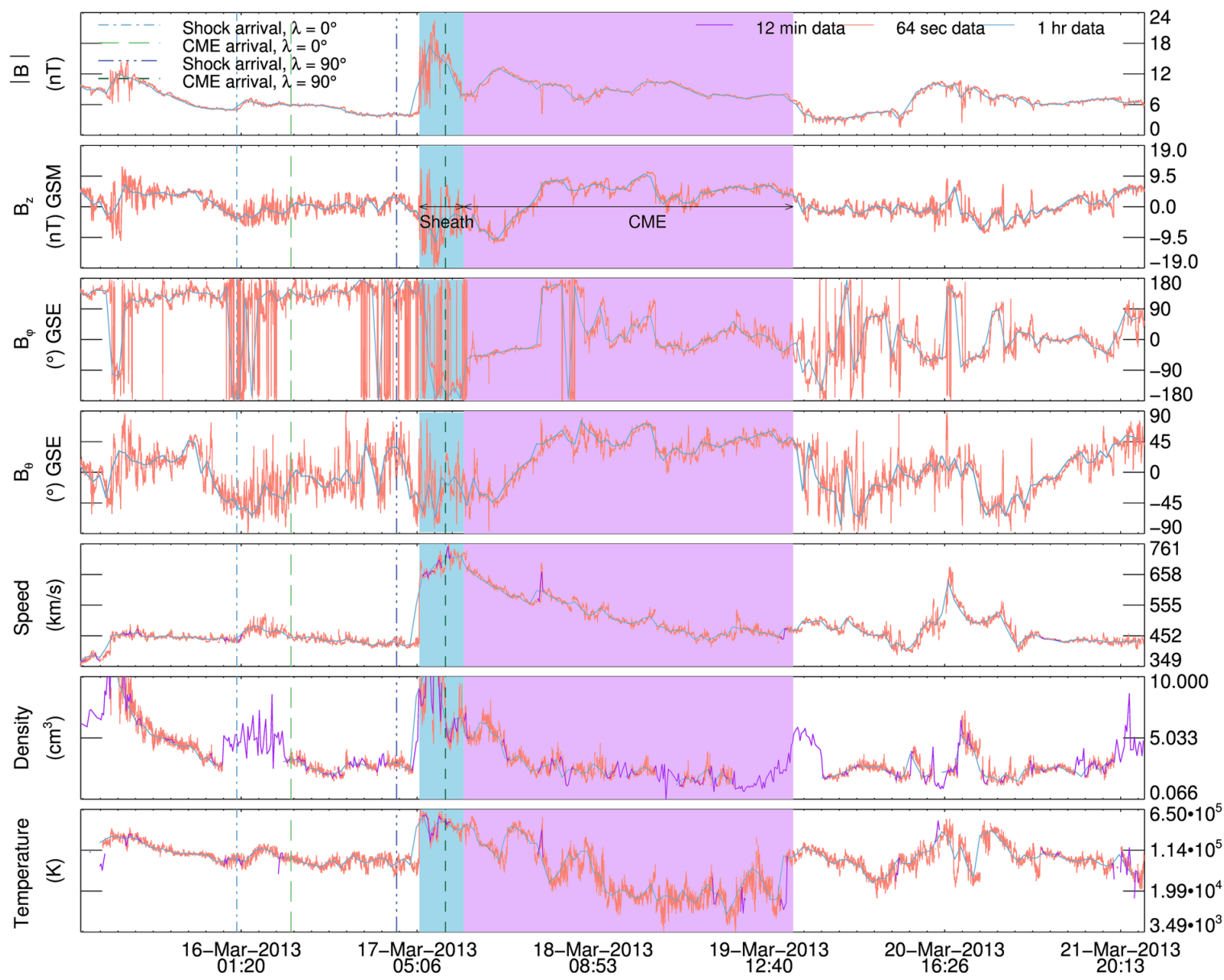

Figure 8.69.: ACE in-situ plasma and magnetic field measurements between 15 and 21 March 2013. The blue area indicates the shock sheath, and the purple area marks the CME passage. Overplotted are the SSSEM arrival times for $\lambda=0^{\circ}$ and $\lambda=90^{\circ}$ obtained via a quadratic fit to the CME and shock SSSEM heights.

\begin{tabular}{llll}
\hline & $\mathrm{ACE}$ & 1st degree fit $\lambda=10^{\circ}$ & 2nd degree fit $\lambda=10^{\circ}$ \\
\hline Standoff distance & $25 R_{\odot}$ & $50 R_{\odot}$ & $52 R_{\odot}$ \\
Compression ratio & 2.87 & 0.29 & 0.27 \\
Mach number & 5.8 & 1.21 & 1.19 \\
\hline & $\mathrm{ACE}$ & 1 st degree fit $\lambda=90^{\circ}$ & 2 nd degree fit $\lambda=90^{\circ}$ \\
\hline Standoff distance & $25 R_{\odot}$ & $62 R_{\odot}$ & $35 R_{\odot}$ \\
Compression ratio & 2.87 & 1.05 & 2.02 \\
Mach number & 5.8 & 1.56 & 2.43 \\
\hline
\end{tabular}

Table 8.30.: Comparison between standoff distance, compression ratio, and Mach number for the 15 March 2013 event as derived from in-situ data, and from the linear and quadratic fit to the SSSEM heights for $\lambda=10^{\circ}$ and $\lambda=90^{\circ}$.

number determined from in-situ data ${ }^{16}$ as well as from the linear and quadratic fits

\footnotetext{
${ }^{16}$ The values of the Mach number and the density compression are available in the HarvardSmithsonian CfA IP shock database (https://www.cfa.harvard.edu/shocks/)
} 
to the SSSEM heights for $\lambda=10^{\circ}$ and $\lambda=90^{\circ}$. Apart from the second degree fit for $\lambda=90^{\circ}$, the values of the standoff distance extrapolated in-situ greatly exceed observations. Independently on whether constant velocity or constant deceleration is assumed, the case $\lambda=10^{\circ}$ yields non physical values for the compression ratio. The best match to observations corresponds to $\lambda=60^{\circ}$ for the linear fit and to $\lambda=90^{\circ}$ for the quadratic fit. These respectively yield $\Delta=61 R_{\odot}, \frac{\rho_{d}}{\rho_{u}}=1.01$ and $M=1.53$, and $\Delta=35 R_{\odot}, \frac{\rho_{d}}{\rho_{u}}=2.20$ and $M=2.43$.

\subsubsection{Corrections for off-Ecliptic propagation}

Table 8.31 shows the relative error associated to the computation of the shock parameters on the Ecliptic plane rather than at the CME apex for $\lambda=60^{\circ}$ and $\lambda=90^{\circ}$, i.e. the SSSEM half-widths associated to the best match between extrapolated and observed standoff distance, compression ratio, and Mach number. Since for all the

\begin{tabular}{lll}
\hline Relative error for & 1st degree fit $\lambda=60^{\circ}$ & 2nd degree fit $\lambda=90^{\circ}$ \\
\hline Standoff distance & $0.4 \%$ & $0.4 \%$ \\
CME curvature & $0.5 \%$ & $0.4 \%$ \\
Compression ratio & $0.07 \%$ & $4 \cdot 10^{-6 \%}$ \\
Mach number & $0.02 \%$ & $3 \cdot 10^{-6 \%}$ \\
\hline
\end{tabular}

Table 8.31.: Relative difference between the standoff distance, the CME radius of curvature, the compression ratio and the Mach number computed on the Ecliptic plane and at the CME apex for the 15 March 2013 event. The results for the value of lambda which yields the best fit to the in-situ shock parameters are shown.

shock parameters and in both the cases of propagation at constant speed and constant deceleration the discrepancy is minimal, the SSSEM can be considered a valid method for for the determination of the shock parameters for this event.

The event presented in this section erupted from AR 11692 on 15 March 2013. It was associated with a M1.1 flare, EUV dimming and wave, and post eruptive arcades. GCS modelling of the CME morphology as observed in COR2 and HI1 images was performed. The extension of the GCS flux rope to the locations imaged by HI1 was not straightforward because of the complex morphology of the CME. According to the GCS results the CME speed decreased from $1221 \mathrm{~km} \mathrm{~s}^{-1}$ between 08:08 and 14:49 UT on 15 March 2013, to $625 \mathrm{~km} \mathrm{~s}^{-1}$ between 14:49 and 18:49 UT. The values of GCS apex heights are not in excellent agreement with the results of the SSSEM.

Signatures of a CME-driven shock were identified in difference images. Such signatures were also present in the j-maps at the elongations observed by HI1. The kinematics of 
the CME and the CME-driven shock could therefore be determined separately. For both features the direction of propagation is compatible with propagation towards Earth. The CME speed shows an initial increase followed by deceleration, whereas the shock speed exhibits a more constant profile.

ACE in-situ data revealed the passage of an interplanetary shock on 17 March 2012 at 05:30, followed by the CME, which hit the spacecraft at 12:30. The best fit to the observed arrival times and speed are obtained for $\lambda=90^{\circ}$ in the case of constant deceleration. The associated arrival time predictions have errors lower than 2 hours, and the shock arrival speed is predicted with an error lower than $8 \%$.

The SSSEM results for the CME and shock kinematics were used to determine the temporal evolution of the shock parameters. The standoff distance was found to increase with time, whereas the Mach number and the compression ratio show roughly constant profiles. The case $\lambda=10^{\circ}$ is associated to density ratios consistently smaller than 1 . The shock parameters were extrapolated to $L_{1}$ and compared to the values determined from ACE data. If the CME and the shock are assumed to propagate at constant speed the best fit is obtained for $\lambda=60^{\circ}$. If the $C M E$ and the shock are assumed to propagate at constant deceleration the best fit corresponds to $\lambda=90^{\circ}$. The latter case yields the most precise results for the shock parameters.

Both the results for the arrival time and for the extrapolations of the shock parameters at $L_{1}$ support the hypothesis that deceleration occurred during the CME the shock interplanetary propagation. 

9. Discussion and conclusions 

The present chapter provides a summary and a discussion of the results obtained from the investigation of STEREO/SECCHI observations of 11 fast CMEs and their associated interplanetary shocks. Suggestions on the improvement of the employed models are provided. Finally, the application of the methods used in this work to future space missions is discussed.

\subsection{On the extension of the GCS model to heliospheric imagers observations}

This work presented the analysis of eleven fast, Earth directed CMEs identified in observations from the instruments on board the NASA STEREO mission (see Chapter 4).

Geometrical modelling was performed for each event by applying the Graduated Cylindrical Shell (GCS, see Chapter 5) model to white-light images. For the events observed before 2012 the modelling results for COR2 observations available on the AFFECTS database $^{1}$ were used. For the other events the morphology observed in the COR2 field of view was fitted with the GCS. The resulting flux ropes were extended to the HI1 field of view, i.e. up to distances of $\simeq 87 R_{\odot}$ in the plane of the sky, in order to determine whether the GCS model can reproduce the geometry of CMEs also in later stages of their propagation. The extension was performed by keeping all the model parameters fixed, and only increasing the CME height. This allows to verify if the assumption of self-similar expansion is applicable to CMEs. The average speeds and radial width of the CME were also estimated. The GCS radial width is defined as the product between the aspect ratio $k$ and the apex height $R_{G C S}, a=k R_{G C S}$. For self similarly expanding flux ropes the radial width $a$ increases at the same rate as the height $R_{G C S}$ by definition.

The extension of the COR2 modelling to HI1 observations was performed for 10 out of the 11 events. The 23 April $2012 \mathrm{CME}$ exhibited a complex morphology not compatible with the GCS geometry. For 4 out of the remaining 10 events the GCS parameters in the HI1 field of view could not be constrained with certainty. In these cases, i.e. the 22 October 2011, 19 January 2012, 23 January 2012 and 03 March 2012 events, the apex location and the projected angular width were such that much of the CME lied outside the field of view of the instrument.

In general, the projected angular width of a feature in white-light observations is described in terms of the feature's extent in position angle (PA). If the heliocentric latitude

\footnotetext{
${ }^{1}$ http://www .affects-fp7 .eu/cme-database/database.php
} 


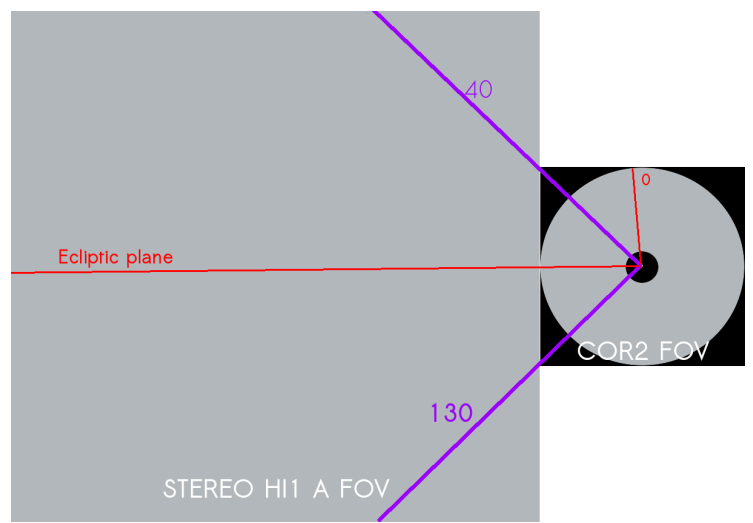

(a)

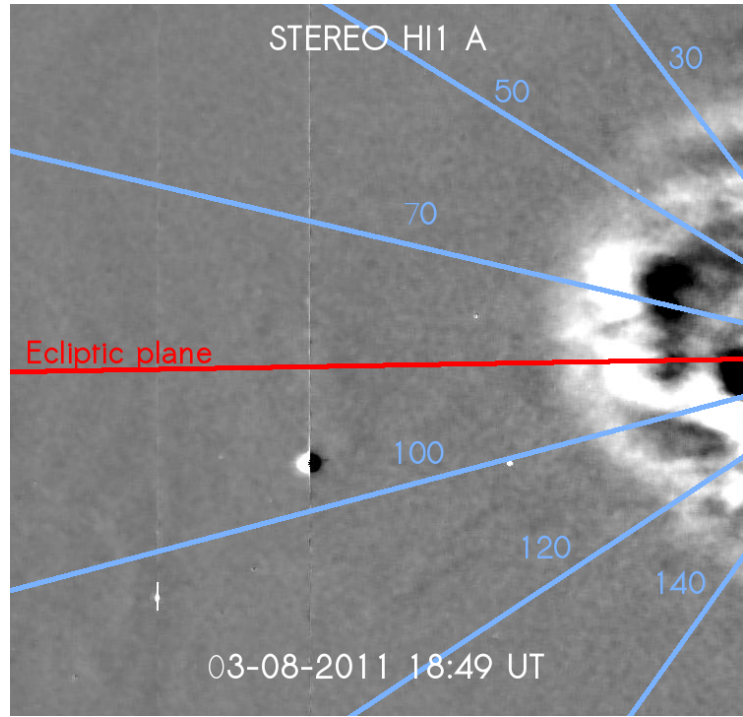

(b)

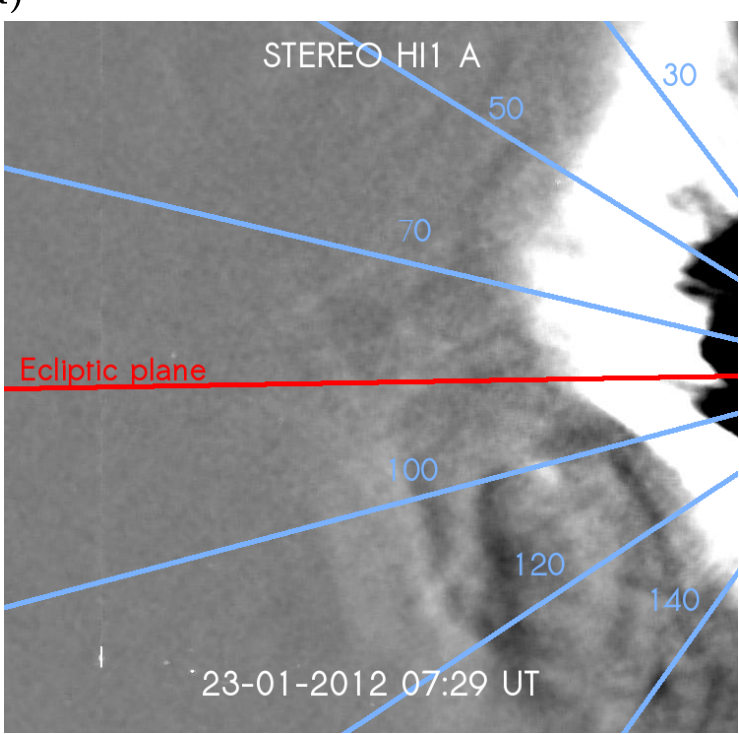

(c)

Figure 9.1.: Top panel: STEREO/SECCHI COR2 and HI1 fields of view showing the opening of the HI1 field of view in terms of position angles (PAs). The Ecliptic plane and the line $P A=0^{\circ}$ are marked in red. Bottom panel: STEREO/SECCHI HI1A difference images of the 03 August 2011 (right) and the 23 January 2012 (left) events. Lines of constant position angle are plotted over the images. Although the apex inclination with respect to the Ecliptic plane (red horizontal line) was similar for the two events, due to their different projected angular width, the 03 August 2011 event was imaged by HI1 in its entirety, while a large fraction of the 23 January 2012 lies outside the field of view of the instrument.

$\phi$ and the elongation $\epsilon$ are known, the position angle can be determined as

$$
\sin (P A)=1-\frac{\sin \phi}{\sin \epsilon}
$$

with $\epsilon$ given by

$$
\cos \epsilon=\cos \phi \cos \theta
$$

(see Webb et al. 2009). The position angle is zero along the solar north and increases counter-clockwise. Equations 9.1 and 9.2 show that the angular span of a CME in terms 
of position angles is ultimately determined by its latitudinal angular size $\Delta \phi$, and the longitude of its apex. Since the field of view of HI1 covers about $90^{\circ}$ in PA (see Figure 9.1a), a CME with half-width $\omega=45^{\circ}$ propagating along the Ecliptic plane will be entirely within the HI1 field of view. CMEs whose apex is at position angle $P A_{A P E X}$ can be fully observed in HI1 if their half-width $\omega$ is such that $\omega<45^{\circ}-P A_{A P E X}$. Figures 9.1b and 9.1c illustrate two CMEs, the 03 August 2011 and the 23 January 2012 as observed by HI1 A. The latitude of the apex as determined via the GCS model was very similar for the two events ${ }^{2}$. The first, though, was imaged in its entirety by HI1, while for the second much of the CME lied outside the field of view of the instrument. As can be readily seen from Figure 9.1, the reason lies within the very different projected angular sizes of the two CMEs. All the events analysed in this work which were not fully within the HI1 field of view had an apex latitude $\phi_{G C S}>25^{\circ}$ and GCS half-width $\alpha>30^{\circ}$.

Average speeds were computed for all the modelled CMEs, although, for the reasons explained above, the most reliable results are those obtained for the 6 CMEs which were fully observed by HI1. In all such cases the speeds were found to decrease as the CME propagated away from the Sun. The average acceleration was between $-15.21 \mathrm{~m} \mathrm{~s}^{-2}$ and $-1.13 \mathrm{~m} \mathrm{~s}^{-2}$. The GCS heights were compared with the ones determined from the analysis of time-elongation plots. Excellent agreement is found for the 12 December 2008, the 03 April 2010 and the 03 August 2011 events, for which the GCS and SSSEM heights differ at most by $13 \%$. In the other cases the values can differ by up to $32 \%$. In comparing the GCS and SSSEM heights one has to take into account that, if the CMEs propagate at large inclinations with respect to the Ecliptic plane, the two methods track very different features, and a discrepancy in the heights is expected, with the GCS yielding the largest values.

Overall the extension of the GCS flux ropes from COR2 to HI1 proved to be quite challenging, since the smooth GCS front was not always appropriate in reproducing the more complex structure observed at higher elongations. Small deviations from the GCS morphology already observed in COR2 expand to even larger scales as the CME moves away from the Sun, thus increasing the discrepancy between model and observations. The difficulty, as already discussed, is increased in those events that have a high off-Ecliptic component in their direction of propagation and a large angular width.

Nevertheless the observed CME structures expanded consistently and individual features could be identified at different stages of the CME propagation for all the analysed events.

It should be noted that some of the difficulties discussed so far in extending the GCS model to higher heliocentric distances might be related to the fact that this analysis was focused on very fast and energetic CMEs. Such events should have a strong interaction with the ambient solar wind leading to distortion and deviations from the ideal flux rope geometry. The results presented here suggest that slower events with small angular extent in white-light images could provide a more appropriate set to test the performance of the GCS model beyond coronagraph images.

\footnotetext{
${ }^{2} \phi_{G C S}=24^{\circ}$ for the 03 August 2011 and $\phi_{G C S}=25^{\circ}$ for the 23 January 2012
} 


\subsection{On the accuracy of the arrival times extrapolations}

STEREO/SECCHI observations were used to derive running difference images from remote-sensing observations of CMEs. In such images the visibility of faint features, like e.g. the signatures of CME-driven shocks, is enhanced. They are the building blocks of time-elongation plots, which in turn allow to track the evolution of propagating solar wind structures at a given position angle (measured anticlockwise from solar north). In this work the evolution on the Ecliptic plane was followed. This allows a comparison between arrival time predictions at $L_{1}$ and ACE plasma and magnetic field data. The SSSEM was applied to time-elongation pairs measured from the j-maps in the range $0^{\circ}<\lambda<90^{\circ}$, with $\lambda$ varying in steps of $10^{\circ}$. The case $\lambda=0^{\circ}$ represents the CME as a point source, whereas the case $\lambda=90^{\circ}$ represents the CME as a circle anchored at the Sun at all times. For the intermediate values the CME is modelled as a circle expanding at constant half-width $\lambda$. The SSSEM yields the temporal evolution of the heliocentric distance, the speed, and the angle of propagation with respect to the Sun-Earth line of solar wind transients. Five out of the 11 events investigated in this study drove interplanetary shocks that were visible in STEREO observations and in time-elongation plots. For those events it was possible to infer the CME and shock kinematics separately. For the other events the evolution of the outermost bright white-light feature is followed. Since in these cases it is not clear whether the evolution of the CME leading edge or of the shock is derived, they are referred to in this work as Interplanetary CMEs (ICMEs). The arrival times and speeds at the location of the ACE spacecraft were computed by performing a linear and a quadratic fit to the CME and, when available, shock heliocentric distances. Their predicted values were compared to ACE data. Figure 9.2 shows the difference between observed and estimated time of arrival, $\Delta t_{A R R}$, for the nine events that were observed in-situ. The plots include the results of both the constant speed and the constant deceleration fit for $\lambda=0^{\circ}$ and $\lambda=90^{\circ}$. The error in the arrival times for the events in which a distinction between CME and shock was not possible, referred to as ICMEs, are shown in purple. For the events in which a separate analysis of CME and shock was possible, the arrival time errors for the two features are plotted next to each other. The blue bar represents the values obtained for the shock, and the pink bar represents the values obtained for the CME. Positive values of $\Delta t_{A R R}$ correspond to predicted arrival times earlier then in-situ arrival.

In 7 out of the 9 presented events the SSSEM results for $\lambda=90^{\circ}$ provided the best accuracy in the arrival time predictions. This, however, should not be interpreted as a general property of the SSSEM in the case $\lambda=90^{\circ}$. Möstl et al. (2014) performed a comparison between the prediction capabilities of single spacecraft fixed- $\phi$ (i.e. $\lambda=0^{\circ}$ ), harmonic mean (i.e. $\lambda=90^{\circ}$ ) and self-similar expansion fitting techniques on a sample of 22 CMEs observed in HI1 and later on by in-situ spacecraft at $L_{1}$. The comparison showed that the three methods possess similar predictive accuracy. To the author's knowledge, however, a similar study has yet to be performed on multi-spacecraft ob- 

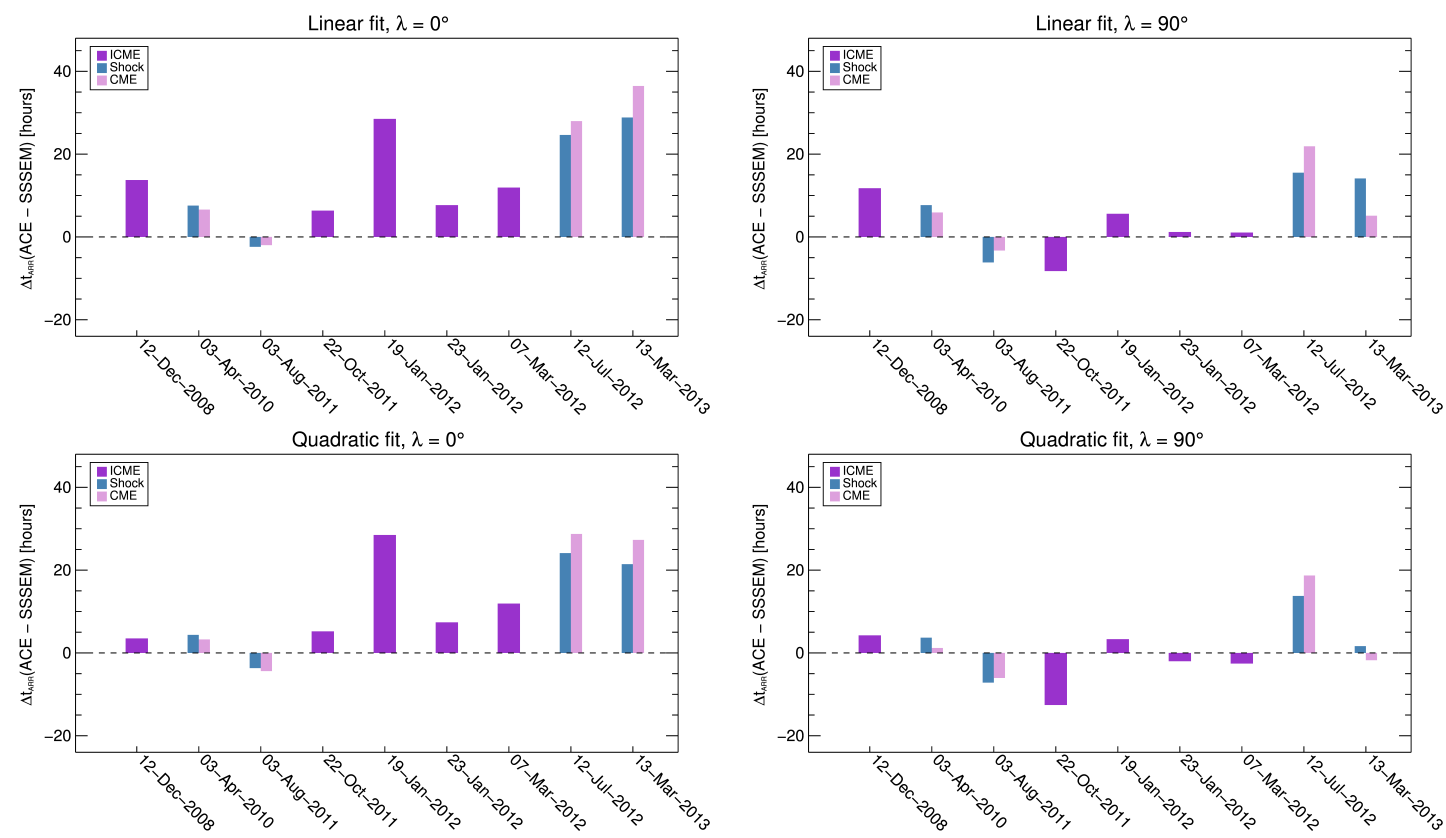

Figure 9.2.: Difference between the observed and predicted arrival times al $L_{1}$ for the 9 events observed in-situ. Positive values correspond to early predictions. Results are presented for the linear and quadratic fits to the CME and, when available, shock height derived from the application of the SSSEM for $\lambda=0^{\circ}$ and $\lambda=90^{\circ}$.

servations of CMEs. Only a consistent analysis on a larger sample of multi-spacecraft observations of CMEs can provide the means to verify whether a certain half-width $\lambda$ is associated to better arrival time predictions.

The inclusion of deceleration in the fits to the SSSEM heights produces more accurate results in 6 out of the 9 events presented here.

For the cases in which the constant speed assumption leads to predicted arrival times which are later than the observed ones, e.g. the 03 August 2011 event, additional deceleration in the model trivially reduces the accuracy of the prediction.

Overall the errors in the estimates for the arrival times are below 36 hours even in the less accurate predictions, and can be as low as just $\simeq 1.3$ hours, as in the case of the 23 January 2012 event. The average discrepancies are shown in Table 9.1.

\begin{tabular}{|c|c|c|}
\hline & 1st degree fit $\lambda=0^{\circ}$ & 1st degree fit $\lambda=90^{\circ}$ \\
\hline$\left\langle\delta_{t}\right\rangle$ & $14 \mathrm{~h} 26^{\prime} \pm 11 \mathrm{~h} 46^{\prime}$ & $4 \mathrm{~h} 46^{\prime} \pm 7 \mathrm{~h} 34^{\prime}$ \\
\hline & 2nd degree fit $\lambda=0$ & 2nd degree fit $\lambda=90^{\circ}$ \\
\hline$\left\langle\delta_{t}\right\rangle$ & $12 \mathrm{~h} 19^{\prime} \pm 12 \mathrm{~h} 21^{\prime}$ & $1 \mathrm{~h} 43^{\prime} \pm 8 \mathrm{~h} 26^{\prime}$ \\
\hline
\end{tabular}

Table 9.1.: Average errors in the arrival time predictions obtained via a linear (top panel) and quadratic (bottom panel) fit to the CME and, when possible, shock height-time profile derived by applying the SSSEM for $\lambda=0^{\circ}$ and $\lambda=90^{\circ}$ to the events analysed in this study. 


\subsection{On the accuracy of the shock parameter extrapolations}

For the events driving shocks detectable in remote-sensing observations and timeelongation plots the SSSEM results were used to determine the temporal evolution of the density compression across the shock and the upstream Mach number. This was performed in conjunction with models which relate the shock standoff distance, normalized to the CME curvature, to the shock parameters. Such models were introduced in Chapter 7. The standoff distance was obtained as the difference between the shock and CME SSSEM heights,

$$
\Delta=R_{\text {SSSEM }}^{\text {Shock }}-R_{\text {SSSEM }}^{C M E},
$$

and the radius of curvature was taken to be the radius of the SSSEM circle. The latter is a function of the SSSEM half-width:

$$
R_{C}=R_{S S S E M}^{C M E} \frac{\sin \lambda}{1+\sin \lambda} .
$$

From these quantities it is possible to determine the compression ratio and the Mach number according to

$$
\begin{aligned}
\frac{\Delta}{R_{C}} & =0.81 \frac{\rho_{u}}{\rho_{d}} \\
\frac{\Delta}{R_{C}} & =0.81 \frac{(\gamma-1) M^{2}+2}{(\gamma+1)\left(M^{2}-1\right)} .
\end{aligned}
$$

The linear and quadratic fits to the CME and shock SSSEM heights were used to determine the values of the standoff distance, the density compression, and the Mach number at $L_{1}$. A comparison with ACE in-situ data was performed to verify the results.

The in-situ standoff distance $\Delta_{A C E}$ was computed by using the observed CME and shock arrival times and the CME speed. In-situ values of the shock parameters derived from ACE and WIND measurements are available on the Harvard Smithsonian CfA IP shock database ${ }^{3}$ and were used in the present work in order to verify the in-situ extrapolations.

Figure 9.3 shows the difference between observed and extrapolated standoff distances normalized to the observed standoff distance, $\frac{\Delta-\Delta_{A C E}}{\Delta_{A C E}}$, for all the shock events that were detected in-situ, i.e. for the 03 April 2010, the 03 August 2011, the 12 July 2012 and the 15 March 2013 events. The plot shows the relative error for each event associated with the value of $\lambda$ which yields the best fits to observations. This corresponds to the smallest value of the error

$$
\operatorname{Err}(\lambda)=\operatorname{abs}\left(\frac{\Delta(\lambda)-\Delta_{A C E}}{\Delta_{A C E}}\right) .
$$

\footnotetext{
${ }^{3}$ https://www.cfa.harvard.edu/shocks/
} 


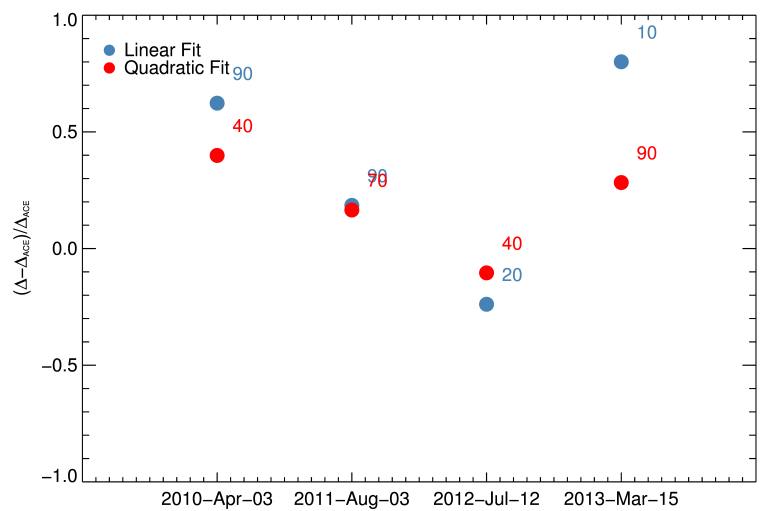

Figure 9.3.: Error for the SSSEM standoff distance normalized to the observed value. Results are reported for the linear and the quadratic fits to the CME and shock SSSEM heights. The plotted values referred to the best fits to observations for the 03 April 2010, the 03 August 2011, the 12 July 2012 and the 15 March 2013 events.

Results are presented for both the linear and quadratic fits to the CME and shock SSSEM heights. The plot shows that assuming deceleration in the shock and CME evolution yields the best estimates for the in-situ standoff distance. The associated errors are of the order of $\simeq 40 \%$ for the 03 April 2010 event, $\simeq 16 \%$ for the 03 August 2011 event, $\simeq 10 \%$ for the 12 July event, and $\simeq 28 \%$ for the 15 March 2013 event. It is important to note that, except for the 03 August 2011 event, in all cases the inclusion of deceleration produces better fits to both the standoff distance and the arrival times. For the 03 August 2011 event the most accurate arrival time prediction is found when constant propagation speed is assume. The standoff distance estimates obtained from the quadratic fit are more accurate than those obtained from the linear fit, but only by $2 \%$. Therefore the results for the accuracy of the standoff distance extrapolations are in agreement with those for the arrival time predictions.

If all the shock parameters are taken into account the best fits to the data is associated to the value of $\lambda$ for which the error

$$
\operatorname{Err}(\lambda)=\sqrt{\left(\frac{\Delta(\lambda)-\Delta_{A C E}}{\Delta_{A C E}}\right)^{2}+\left(\frac{X(\lambda)-X_{A C E}}{X_{A C E}}\right)^{2}+\left(\frac{M(\lambda)-M_{A C E}}{M_{A C E}}\right)^{2}},
$$

with $X=\frac{\rho_{d}}{\rho_{u}}$, is smallest. The values of $\lambda$ associated to non-physical compression ratios $X<1$ were discarded from the evaluation of the best fits.

Figure 9.4 shows the relative error between the observed and extrapolated shock parameters for the four events driving a shock which were detected in-situ. The error is normalized to the in-situ value. The results corresponding to the value of $\lambda$ which yields the best fits to observations according to Equation 9.7 are reported for each event.

In this case the most accurate results for the standoff distance were obtained by assuming that the CME and the shock propagate at constant deceleration, i.e. for the 

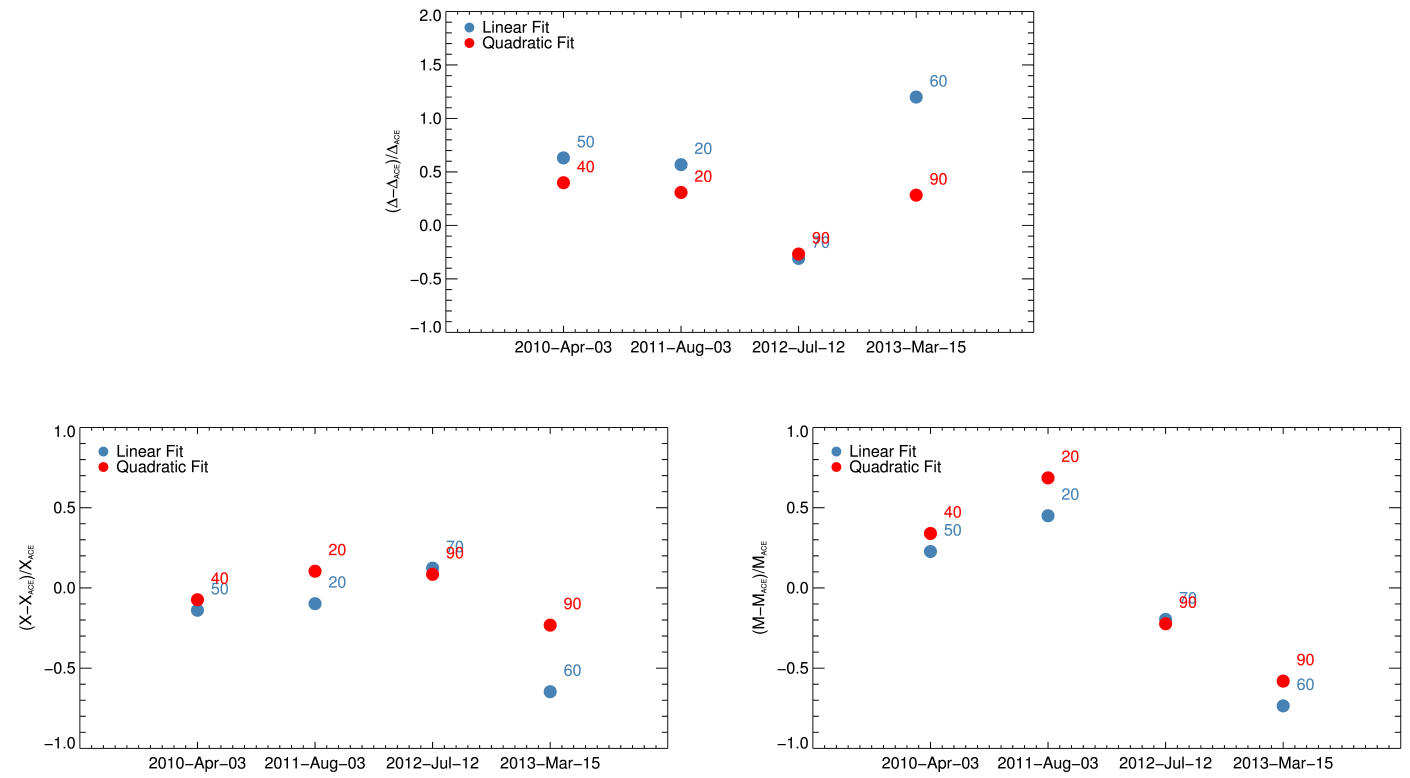

Figure 9.4.: Relative error for the standoff distance, compression ratio, and Mach number compared to observations. Results are reported for the linear and the quadratic fits to the CME and shock SSSEM heights. The plotted values referred to the best fits to observations for the 03 April 2010, the 03 August 2011, the 12 July 2012 and the 15 March 2013 events.

quadratic fits to the CME and shock heights. The corresponding errors are again below $40 \%$.

Except for the linear fit to the CME and shock heights for the 15 March 2013 events, the errors in the extrapolated compression ratio are below $25 \%$, and can be as low as 7\%. For 3 out of the 4 events presented here, namely the 03 April 2010, the 03 August 2011, and the 12 July 2012 events, no striking superiority is found between the linear and quadratic fits; in absolute value they yield comparable accuracies. This statement does not hold for the 15 March 2013 event, for which the precision of the quadratic fit is higher than the one for the linear fit by roughly $40 \%$.

The Mach number extrapolations exhibits higher discrepancies compared to the compression ratio and the standoff distance, ranging from $\simeq 19 \%$ to $\simeq 70 \%$. The linear fit is found to produce the most accurate results in all cases apart from the 15 March 2013 event. Overall, the smallest fitting residues are obtained when assuming that the CME and the shock propagate at constant deceleration in all cases except for the 03 August 2011 CME.

\subsection{Conclusions and Outlook}

The present chapter summarized the results of the analysis of STEREO/SECCHI observations of eleven fast CMEs, five of which drove shocks with signatures detectable in white-light observations and j-maps. Geometrical modelling of the CMEs via the GCS model shows that: 
- the geometry of CMEs is preserved during their interplanetary propagation. Individual features observed in coronagraph images can be followed and identified throughout the evolution of the CMEs in the inner heliosphere;

- the GCS model is most appropriate to reproduce the CME geometry in the later stages of propagation for the slower events whose projected half-width $\omega$ and apex position angle $P A_{A P E X}$ satisfy the condition $\omega+P A_{A P E X}<45^{\circ}$;

- the CME apex speeds was found to decrease as the CMEs travel away from the Sun. The corresponding average accelerations vary between $-15.21 \mathrm{~m} \mathrm{~s}^{-2}$ and $-1.13 \mathrm{~m} \mathrm{~s}^{-2}$

The investigation of STEREO/SECCHI observations and the application of the SSSEM, moreover, indicates that:

- it is possible to independently track the evolution of CMEs and CME-driven shock in the inner heliosphere by means of remote-sensing observations of coronagraph and heliospheric imagers, i.e. up to $\simeq 87 R_{\odot}$ in the plane-of-the-sky;

- inverse modelling techniques can be employed to determine the CME and shock kinematics, and separately infer their arrival times and speeds at Earth. The arrival time precision can be as good as 1.3 hours;

- it is possible to determine the interplanetary evolution of the shock standoff distance, compression ratio, and Mach number by combining the results for the CME and shock kinematics with models for the shock location. To the best of the author's knowledge this is the first time that inverse modelling techniques are employed for the independent determination of the evolution of CMEs and their driven shocks, as well as of the shock parameters.

Finally, the analysis of these results and the comparison to ACE in-situ data gathered at $L_{1}$ shows that:

- multipoint observations are crucial in the achievement of a sufficient level of precision for the determination of the shock parameters from STEREO/SECCHI observations. The analysis of the 07 June 2011 event, based on single spacecraft fitting techniques, showed that the errors in the derived shock parameters are significantly higher than those obtained from models based on multi-spacecraft observations;

- the inclusion of deceleration in the fits to the CME and, when possible, shock height-time profiles improved the accuracy of the arrival time estimates in over $66 \%$ of the cases;

- for all the shock events the standoff distance was found to regularly increase with time, whereas the compression ratio and the Mach number exhibit a more constant profile; 

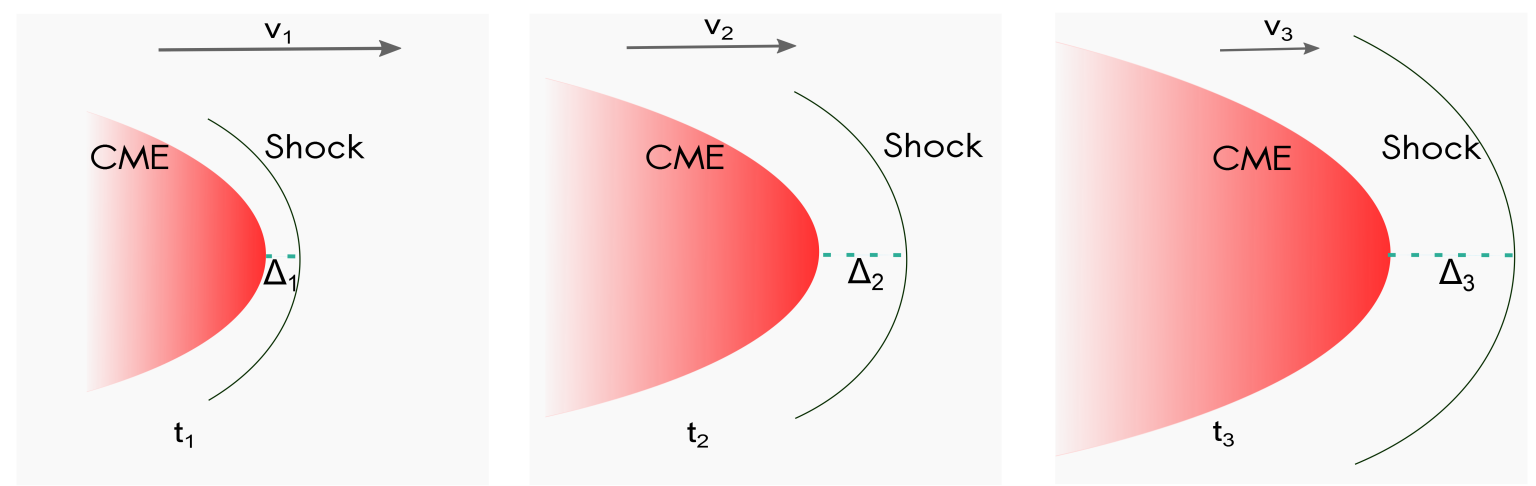

Figure 9.5.: Sketch representing a CME and its driven shock in different stages of their evolution. The picture summarises the results found in this work, namely that the standoff distance increases as the CME travels away from the Sun, and that deceleration occurs during its interplanetary propagation.

- the precision in the extrapolation of the standoff distance also increases if the CME and the shock are assumed to propagate at constant deceleration. The highest errors in this case of the order of $40 \%$, whereas in the best case scenario (i.e. for the 12 July 2012 event) they can be as low as 10\%;

- the density compression ratio can be estimated with errors as low as $7 \%$. The linear and quadratic fit to the SSSEM heights produce comparable accuracies, of the order of $\simeq 10 \%$. An exception is the 15 March 2013 event, for which the errors associated to the quadratic fits are $\simeq 40 \%$ lower than those associated to the linear fit;

- the errors in the Mach number extrapolations, ranging between $\simeq 19 \%$ and $\simeq$ $70 \%$, are higher than those found for the other shock parameters. In three out of the four analysed cases the linear fit yields more accurate predictions for the Mach number.

It is important at this point to make some remarks on the precision of the shock parameters determined in this work.

The analysis of the errors associated to the shock parameters showed that the Mach number extrapolations yield less accurate results compared to the standoff distance and compression ratio. While the errors associated to the shock standoff distance depend solely on the accuracy of the models for the CME and shock kinematics, those associated to the compression ratio and the Mach number depend also on the assumed CME geometry via the CME radius of curvature. Such errors could in principle be reduced by modifying the assumptions on the CME geometry. By using the SSSEM radius of curvature in Equation 9.4, in fact, the CME is represented as a spherical structure. A more sophisticated approach would be to use the curvature of the GCS flux rope determined from fits to COR2 observations.

One could argue, of course, that the assumptions adopted in deriving an equation for the MHD Mach number from purely hydrodynamics relations are the cause for the 


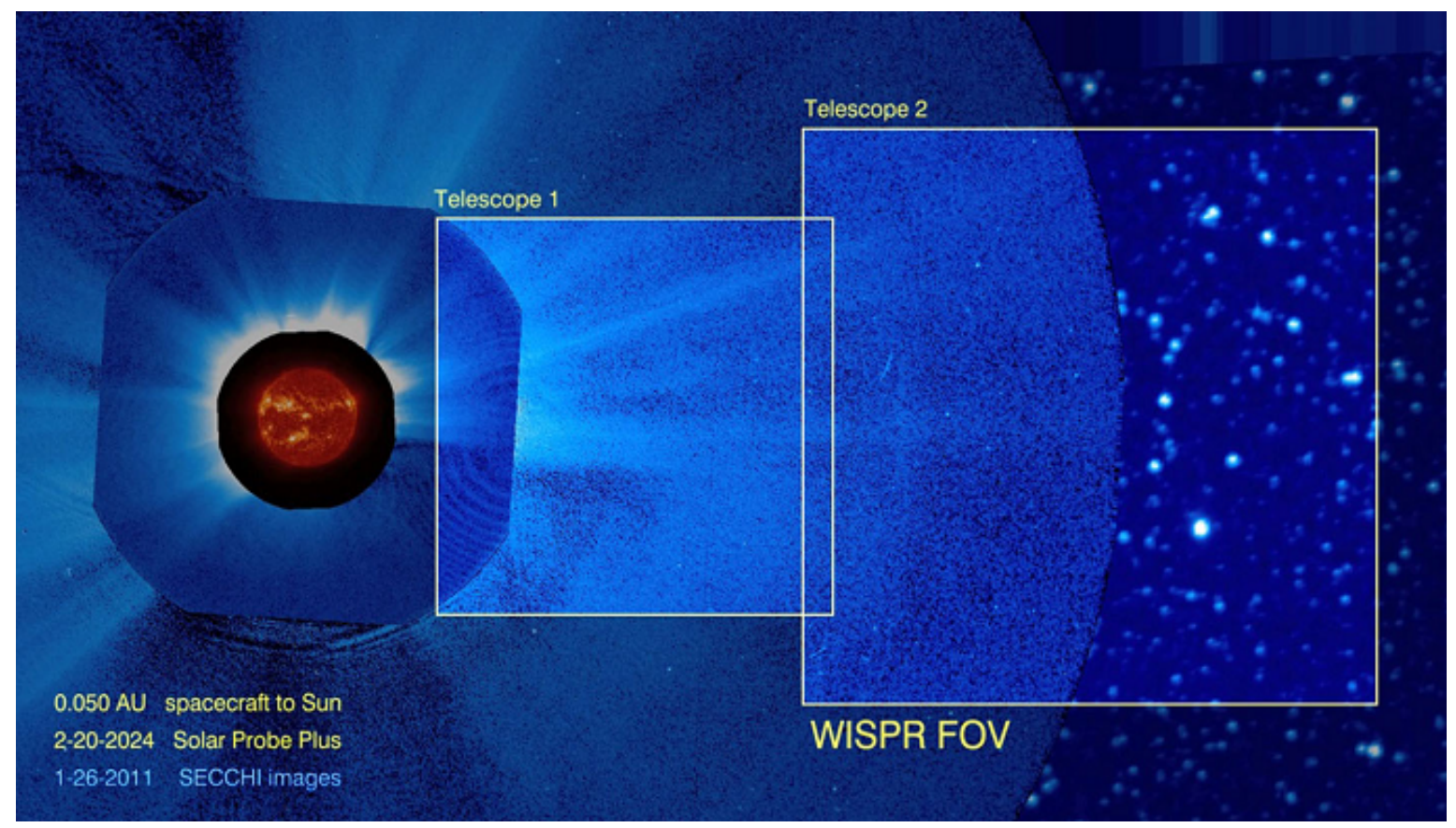

Figure 9.6.: Field of view of the WISPR cameras at 9.86 AU superimposed on a composite STEREO/SECCHI COR and HI1 images. Reprinted from Vourlidas et al. (2015) with the permission of AIP publishing

higher errors in the Mach number extrapolations. In this hypothesis, the better precision achieved for the compression ratio could be ascribed to the fact that its expression is derived solely from mass conservation arguments, and involves one level of approximation less.

The appropriateness of the models for the shock parameter in the context of CMEs, however, can only be assessed if a statistically significant number of events is analysed. This will be possible with the advent of new space based missions like the ESA Solar Orbiter (Müller et al., 2013) and the NASA Solar Probe Plus (Fox et al., 2015), both to be launched in 2018.

The Solar Orbiter spacecraft will reach its operational orbit, with closest perihelion at $0.28 \mathrm{AU}$ and a maximum inclination with respect to the Ecliptic plane of $25^{\circ}$, via the use of several gravity assists at Earth and Venus. It will be equipped with imaging and in-situ instruments. The solar and heliospheric imager (SoloHI Howard et al. 2013) on board Solar Orbiter, with its $40^{\circ}$ wide field of view starting at $5^{\circ}$ from Sun centre, will for the first time image the corona and inner heliosphere from above the Ecliptic plane. It will provide a link between the EUV and in-situ data gathered by the other instruments on board Solar Orbiter.

The Solar Probe Plus mission will fly as close as $8.5 R_{\odot}$ from the Sun via the use of multiple gravity assists at Venus. Remote-sensing and in-situ data will be gathered from 8.5 to at least $53.7 R_{\odot}$ (Fox et al., 2015). The Wide-Field Imager for Solar Probe Plus (WISPR, Vourlidas et al. (2015)) will image the inner heliosphere 
in white-light. The field of view, centred on the ecliptic plane, will extend from $13.5^{\circ}$ to $108^{\circ}$ in elongation. The camera on board WISPR will provide high cadence, high resolution observations of the solar wind and of solar wind transients: in the phases of the mission when the spacecraft will be located within $15 R_{\odot}$ from the Sun, for example, the cadence of 5-10 minutes will provide 13-26 images of a CME travelling at $2000 \mathrm{~km} \mathrm{~s}^{-1}$. This, together with the high resolution achieved by the WISPR cameras, will enable the detection of CME-driven shocks and will provide a large data set for the implementation and testing of the methods present presented in this work. The results presented in this work will help to improve the operations of the WISPR camera developed by the University of Göttingen as German contribution to the NASA Solar Probe Plus mission. Furthermore, the application of the techniques introduced here to remote-sensing observations by SoloHI and WISPR will yield an insight on the spatial dependence of CME-driven shock parameters for a large sample of events. The combination of such results with the information on local plasma and magnetic field properties obtained via the in-situ instruments on board both spacecraft will provide a unique tool to verify the results and to test the validity of the models for the shock location in the context of CMEs. 
Appendices 



\section{A. Error propagation}

The errors associated to the height and direction of propagation obtained via the application of the SSSEM to time-elongation plots are computed following the approach of Liu et al. (2010b). Since the spacecraft STEREO A and B independently measure the elongation angles $\epsilon_{A}$ and $\epsilon_{B}$, the errors associated to the height and the direction of propagation can be written as

$$
\begin{gathered}
\sigma_{R}=\sqrt{\left(\frac{\partial R}{\partial \epsilon_{A}}\right) \sigma_{\epsilon_{A}}^{2}+\left(\frac{\partial R}{\partial \epsilon_{B}}\right) \sigma_{\epsilon_{B}}^{2}}, \\
\sigma_{\phi_{A}}=\sqrt{\left(\frac{\partial \phi_{A}}{\partial \epsilon_{A}}\right) \sigma_{\epsilon_{A}}^{2}+\left(\frac{\partial \phi_{A}}{\partial \epsilon_{B}}\right) \sigma_{\epsilon_{B}}^{2}} .
\end{gathered}
$$

In this work an uncertainty of 10 pixels is assigned to the elongation measurements. This corresponds to $0.04^{\circ}$ in COR2, $0.2^{\circ}$ in HI1, and $0.7^{\circ}$ in HI2. The expressions for $R_{A}$ and $\phi_{A}$ are given in Chapter 6 , but are reported here for completeness (also see Davies et al. (2013)). Theu are

$$
\begin{gathered}
R=\frac{d_{A} \sin \epsilon_{A}(1+\sin \lambda)}{\sin \left(\epsilon_{A}+\phi_{A}\right)+\sin (\lambda)} \\
\sin \phi_{A}=\frac{M \cos \phi_{A}+N}{L}
\end{gathered}
$$

and

$$
\cos \phi_{A}=\frac{-M N \pm \sqrt{L^{2}+M^{2}-N^{2}}}{L^{2}+M^{2}}
$$

from which follows that

$$
\tan \phi_{A}=\frac{L N \pm M \sqrt{L^{2}+M^{2}-N^{2}}}{-M N+L \sqrt{L^{2}+M^{2}-N^{2}}} .
$$

The quantities $L, M$ and $N$ are defined in (Davies et al., 2013) and given by:

$$
\begin{gathered}
L=\cos \left(\epsilon_{B}+\gamma\right)+P \cos \epsilon_{A}, \\
M=\sin \left(\epsilon_{B}+\gamma\right)-P \sin \epsilon_{A}, \\
N=(1-P) \sin \lambda,
\end{gathered}
$$


with

$$
P=\frac{d_{B} \sin \epsilon_{B}}{d_{A} \sin \epsilon_{A}}
$$

and

$$
\gamma=\phi_{A}+\phi_{B}
$$

As discussed in Chapter 6 two possible solutions for $\tan \phi_{A}$ exist, and it is necessary to discern which one is really representative of the system under study. For the purposes of this discussions they will be referred to as positive and negative solutions, in reference to the sign before the $M \sqrt{L^{2}+M^{2}-N^{2}}$ term in Equation A.6.

Keeping the above definitions in mind, the derivatives in Equations A.1 and A.2 can be expanded to get

$$
\begin{gathered}
\frac{\partial R}{\partial \epsilon_{A}}=\frac{d_{A} \sin \lambda\left(\sin \lambda \cos \epsilon_{A}+\sin \phi_{A}-\cos \left(\epsilon_{A}+\phi_{A}\right) \sin \epsilon_{A} \frac{\partial \phi_{A}}{\partial \epsilon_{A}}\right)}{\left(\sin \left(\epsilon_{A}+\phi_{A}\right)\right)+\sin \lambda} ; \\
\frac{\partial R}{\partial \epsilon_{B}}=-\frac{d_{A} \sin \lambda \cos \left(\epsilon_{A}+\phi_{A}\right) \sin \epsilon_{A} \frac{\partial \phi_{A}}{\partial \epsilon_{B}}}{\left(\sin \left(\epsilon_{A}+\phi_{A}\right)\right)+\sin \lambda}
\end{gathered}
$$

For the error associated to the direction of propagation, the definition in Equation A.6 is used together with the fact that

$$
\sin ^{2} \phi_{A} \frac{\partial \phi_{A}}{\partial \epsilon_{A}}=\frac{\partial \tan \phi_{A}}{\partial \epsilon_{A}}
$$

with $\sin \phi_{A}$ given in Equation A.4.We get:

$$
\begin{aligned}
& \frac{\partial \tan \phi_{A}}{\partial \epsilon_{A}}=\frac{ \pm\left(L^{2}+M^{2}\right)\left( \pm L\left(\mp N \frac{\partial L}{\partial \epsilon_{A}}+\sqrt{L^{2}+M^{2}-N^{2}}\right)\right.}{\sqrt{L^{2}+M^{2}-N^{2}}\left(M N \mp L \sqrt{L^{2}+M^{2}-N^{2}}\right)}+ \\
& \frac{\left.L^{2} \frac{\partial N}{\partial \epsilon_{A}}+M\left(\mp \sqrt{L^{2}+M^{2}-N^{2}} \frac{\partial L}{\partial \epsilon_{A}}-N \frac{\partial M}{\partial \epsilon_{A}}+M \frac{\partial N}{\partial \epsilon_{A}}\right)\right)}{\sqrt{L^{2}+M^{2}-N^{2}}\left(M N \mp L \sqrt{L^{2}+M^{2}-N^{2}}\right)}
\end{aligned}
$$

and

$$
\begin{aligned}
\frac{\partial \tan \phi_{A}}{\partial \epsilon_{B}}= & \frac{ \pm\left(L^{2}+M^{2}\right)\left( \pm L\left(\mp N \frac{\partial L}{\partial \epsilon_{B}}+\sqrt{L^{2}+M^{2}-N^{2}}\right)\right.}{\sqrt{L^{2}+M^{2}-N^{2}}\left(M N \mp L \sqrt{L^{2}+M^{2}-N^{2}}\right)}+ \\
& \frac{\left.L^{2} \frac{\partial N}{\partial \epsilon_{B}}+M\left(\mp \sqrt{L^{2}+M^{2}-N^{2}} \frac{\partial L}{\partial \epsilon_{B}}-N \frac{\partial M}{\partial \epsilon_{B}}+M \frac{\partial N}{\partial \epsilon_{B}}\right)\right)}{\sqrt{L^{2}+M^{2}-N^{2}}\left(M N \mp L \sqrt{L^{2}+M^{2}-N^{2}}\right)}
\end{aligned}
$$

with the double sign referring to the positive and negative roots of Equation A.6. Finally, the derivatives of the coefficients $L, M$ and $N$ are: 


$$
\begin{gathered}
\frac{\partial L}{\partial \epsilon_{A}}=-\frac{d_{B} \csc ^{2} \epsilon_{A} \sin \epsilon_{B}}{d_{A}} \\
\frac{\partial L}{\partial \epsilon_{B}}=\frac{d_{B} \cos \epsilon_{B} \cot \epsilon_{A}}{d_{A}}-\sin \left(\epsilon_{B}+\gamma\right), \\
\frac{\partial M}{\partial \epsilon_{A}}=0 \\
\frac{\partial M}{\partial \epsilon_{B}}=-\frac{d_{B} \cos \epsilon_{B}}{d_{A}}+\cos \left(\epsilon_{B}+\gamma\right) \\
\frac{\partial N}{\partial \epsilon_{A}}=-\frac{d_{B} \sin \lambda \cot \epsilon_{A} \csc \epsilon_{A} \sin \epsilon_{B}}{d_{A}} \\
\frac{\partial N}{\partial \epsilon_{B}}=-\frac{d_{B} \sin \lambda \cos \epsilon_{B} \csc \epsilon_{A}}{d_{A}}
\end{gathered}
$$

The equations presented so far allow the determination of the errors associated to the height and direction of propagation obtained via the SSSEM model from the uncertainties in the measured elongation angles. The error associated to the speed is:

$$
\sigma_{v}=\frac{v}{R} \sigma_{R}
$$

The quantities derived from the application of the SSSEM are used to compute the shock standoff distance, compression ratio, and Mach number through the following relations:

$$
\begin{aligned}
\Delta & =R_{S h o c k}-R_{C M E} \\
R_{C} & =R_{C M E} \frac{\sin \lambda}{1+\sin \lambda} \\
\frac{\Delta}{R_{C}} & =0.81 \frac{\rho_{u}}{\rho_{d}} \\
\frac{\Delta}{R_{C}} & =0.81 \frac{(\gamma-1) M^{2}+2}{(\gamma+1)\left(M^{2}-1\right)},
\end{aligned}
$$

with $\Delta$ being the standoff distance, $R_{\text {Shock }}$ and $R_{C M E}$ shock and CME heliocentric distances as derived from the SSSEM, $R_{C}$ the CME radius of curvature, $\rho_{u}$ and $\rho_{d}$ the upstream and downstream densities, and $M$ the Mach number. For the rest of this derivation $Y \equiv \frac{\Delta}{R_{C}}$. The errors associated to the shock parameters were computed using the approach described so far. They are:

$$
\begin{gathered}
\sigma_{\Delta}=\sqrt{\sigma_{R_{S h o c k}^{2}}^{2}+\sigma_{R_{C M E}^{2}}^{2}}, \\
\sigma_{R_{C}}=\frac{R_{C}}{R_{C M E}} \sigma_{R C M E},
\end{gathered}
$$




$$
\begin{gathered}
\sigma_{Y}=\sqrt{\left(\frac{\sigma_{\Delta}}{R_{C}}\right)^{2}+\left(\frac{\Delta}{R_{C}^{2}} \sigma_{R_{C}}^{2}\right)^{2}}, \\
\sigma_{\frac{\rho_{u}}{\rho_{d}}}=\frac{1}{Y^{2}} \sigma_{Y},
\end{gathered}
$$

and

$$
\sigma_{\text {Mach }}=\frac{4}{4 Y-0.81} \frac{1-M^{2}}{2 M} \sigma_{Y}
$$




\section{Bibliography}

Antiochos, S. K., DeVore, C. R. \& Klimchuk, J. A. (1999) A Model for Solar Coronal Mass Ejections. Astrophys. J., 510, 485-493.

Bateman, G. (1978) MHD Instabilities. MIT Press, Cambridge, MA.

Baumjohann, W. \& Treumann, R. A. (1996) Basic space plasma physics. London: Imperial College Press.

Benz, A. O. (2008) Flare Observations. Living Reviews in Solar Physics, 5.

Bosman, E., Bothmer, V., Nisticò, G., Vourlidas, A., Howard, R. A. \& Davies, J. A. (2012) Three-Dimensional Properties of Coronal Mass Ejections from STEREO/SECCHI Observations. Solar Phys., 281, 167-185.

Bothmer, V. (1999) Magnetic field structure and topology within CMEs in the solar wind. In American Institute of Physics Conference Series, edited by S. T. Suess, G. A. Gary \& S. F. Nerney, vol. 471 of American Institute of Physics Conference Series.

Bothmer, V. (2004) The Solar and Interplanetary Causes of Space Storms in Solar Cycle 23. IEEE Transactions on Plasma Science, 32, 1411-1414.

Bothmer, V. \& Daglis, I. A. (2007) Space Weather - Physics and Effects. Springer Berlin Heidelberg.

Bothmer, V. \& Schwenn, R. (1994) Eruptive prominences as sources of magnetic clouds in the solar wind. Space Sci. Rev., 70, 215-220.

Bothmer, V. \& Schwenn, R. (1995) The Interplanetary and Solar Causes of Major Geomagnetic Storms. Earth, Planets and Space, 47(11), 1127-1132, URL http: //ci.nii.ac.jp/naid/130003423098/en/.

Bothmer, V. \& Schwenn, R. (1996) Signatures of fast CMEs in interplanetary space. Advances in Space Research, 17(4-5), 319 - 322.

Bothmer, V. \& Schwenn, R. (1998) The structure and origin of magnetic clouds in the solar wind. Annales Geophysicae, 16, 1-24.

Bougeret, J. L., Goetz, K., Kaiser, M. L., Bale, S. D., Kellogg, P. J., Maksimovic, M., Monge, N., Monson, S. J. ET AL. (2008) S/WAVES: The Radio and Plasma Wave Investigation on the STEREO Mission. Space Sci. Rev., 136, $487-528$. 
Burlaga, L., Sittler, E., Mariani, F. \& Schwenn, R. (1981) Magnetic loop behind an interplanetary shock - Voyager, Helios, and IMP 8 observations. J. Geophys. Res., 86, 6673-6684.

Byrne, J. P., Maloney, S. A., McAteer, R. T. J., Refojo, J. M. \& GalLAGHER, P. T. (2010) Propagation of an Earth-directed coronal mass ejection in three dimensions. Nature Communications, 1, 74 .

Cane, H. V. \& Richardson, I. G. (2003) Interplanetary coronal mass ejections in the near-Earth solar wind during 1996-2002. Journal of Geophysical Research (Space Physics), 108, 1156.

Cane, H. V., Sheeley, JR., N. R. \& Howard, R. A. (1987) Energetic interplanetary shocks, radio emission, and coronal mass ejections. J. Geophys. Res., 92, 9869-9874.

Cargill, P. J. (2004) On the Aerodynamic Drag Force Acting on Interplanetary Coronal Mass Ejections. Solar Phys., 221, 135-149.

Carmichael, H. (1964) A Process for Flares. NASA Special Publication, 50, 451.

Chen, J. (1989) Effects of toroidal forces in current loops embedded in a background plasma. Astrophys. J., 338, 453-470.

Chen, J. (1996) Theory of prominence eruption and propagation: Interplanetary consequences. J. Geophys. Res., 101, 27499-27520.

Chen, J., Howard, R. A., Brueckner, G. E., Santoro, R., Krall, J., PaswaTers, S. E., St. Cyr, O. C., Schwenn, R. ET AL. (1997) Evidence of an Erupting Magnetic Flux Rope: LASCO Coronal Mass Ejection of 1997 April 13. Astrophys. J. Lett., 490, L191-L194.

Chen, J., Santoro, R. A., Krall, J., Howard, R. A., Duffin, R., Moses, J. D., Brueckner, G. E., Darnell, J. A. et AL. (2000) Magnetic Geometry and Dynamics of the Fast Coronal Mass Ejection of 1997 September 9. Astrophys. J., 533, 481-500.

Chen, P. F. (2011) Coronal Mass Ejections: Models and Their Observational Basis. Living Reviews in Solar Physics, 8(1), URL http://www.livingreviews.org/ lrsp-2011-1.

Chen, P. F., Fang, C. \& Shibata, K. (2005) A Full View of EIT Waves. Astrophys. J., 622, 1202-1210.

Chen, P. F. \& Shibata, K. (2000) An Emerging Flux Trigger Mechanism for Coronal Mass Ejections. Astrophys. J., 545, 524-531. 
Colaninno, R. C., Vourlidas, A. \& Wu, C. C. (2013) Quantitative comparison of methods for predicting the arrival of coronal mass ejections at Earth based on multiview imaging. Journal of Geophysical Research (Space Physics), 118, 68666879 .

Cremades, H. \& Bothmer, V. (2004) On the three-dimensional configuration of coronal mass ejections. Astron. Astrophys., 422, 307-322.

Cremades, H. \& Bothmer, V. (2005a) Geometrical Properties of Coronal Mass Ejections. In Coronal and Stellar Mass Ejections, edited by K. Dere, J. Wang \& Y. Yan, vol. 226 of IAU Symposium.

Cremades, H. \& Bothmer, V. (2005b) Three-Dimensional Configuration and Evolution of Coronal Mass Ejections. Ph.D. thesis, Technischen Universität CaroloWilhelmina, Braunschweig.

Davies, J. A., Harrison, R. A., Perry, C. H., Möstl, C., Lugaz, N., Rollett, T., Davis, C. J., Crothers, S. R. Et Al. (2012) A Self-similar Expansion Model for Use in Solar Wind Transient Propagation Studies. Astrophys. J., 750, 23.

Davies, J. A., Harrison, R. A., Roulllard, A. P., Sheeley, N. R., Perry, C. H., Bewsher, D., Davis, C. J., Eyles, C. J. et Al. (2009) A synoptic view of solar transient evolution in the inner heliosphere using the Heliospheric Imagers on STEREO. Geophys. Res. Lett., 36, 2102.

Davies, J. A., Perry, C. H., Trines, R. M. G. M., Harrison, R. A., Lugaz, N., Möstl, C., Liu, Y. D. \& Steed, K. (2013) Establishing a Stereoscopic Technique for Determining the Kinematic Properties of Solar Wind Transients based on a Generalized Self-similarly Expanding Circular Geometry. Astrophys. J., 777, 167.

Davis, C. J., Davies, J. A., Lockwood, M., Rouillard, A. P., Eyles, C. J. \& HARRISON, R. A. (2009) Stereoscopic imaging of an Earth-impacting solar coronal mass ejection: A major milestone for the STEREO mission. Geophys. Res. Lett., 36, 8102 .

DeForest, C. E., Howard, T. A. \& McComas, D. J. (2013) Tracking Coronal Features from the Low Corona to Earth: A Quantitative Analysis of the 2008 December 12 Coronal Mass Ejection. Astrophys. J., 769(1), 43, URL http: //stacks.iop.org/0004-637X/769/i=1/a=43.

Dryer, M. \& SMART, D. F. (1984) Dynamical models of coronal transients and interplanetary disturbances. Advances in Space Research, 4, 291-301.

Eyles, C. J., Harrison, R. A., Davis, C. J., Waltham, N. R., Shaughnessy, B. M., Mapson-Menard, H. C. A., Bewsher, D., Crothers, S. R. et Al. (2009) The Heliospheric Imagers Onboard the STEREO Mission. Solar Phys., 254, $387-445$. 
Fairfield, D. H., Cairns, I. H., Desch, M. D., Szabo, A., Lazarus, A. J. \& Aellig, M. R. (2001) The location of low Mach number bow shocks at Earth. J. Geophys. Res., 106, 25361-25376.

FARris, M. H. \& Russell, C. T. (1994) Determining the standoff distance of the bow shock: Mach number dependence and use of models. J. Geophys. Res., 99, 17681.

Feynman, J. \& Martin, S. F. (1995) The initiation of coronal mass ejections by newly emerging magnetic flux. J. Geophys. Res., 100, 3355-3367.

Forbes, T. G. (2000) A review on the genesis of coronal mass ejections. J. Geophys. Res., 105, 23153-23166.

Fox, N. J., Velli, M. C., Bale, S. D., Decker, R., Driesman, A., Howard, R. A., Kasper, J. C., Kinnison, J. Et Al. (2015) The Solar Probe Plus Mission: Humanity's First Visit to Our Star. Space Sci. Rev..

Galvin, A. B., Kistler, L. M., Popecki, M. A., Farrugia, C. J., Simunac, K. D. C., Ellis, L., Möbius, E., Lee, M. A. ET AL. (2008) The Plasma and Suprathermal Ion Composition (PLASTIC) Investigation on the STEREO Observatories. Space Sci. Rev., 136, 437-486.

Gargaté, L., Fonseca, R. A., Silva, L. O., Bamford, R. A. \& Bingham, R. (2014) SEP Acceleration in CME Driven Shocks Using a Hybrid Code. Astrophys. J., $792,9$.

Goldstein, H. (1983) On the field configuration in magnetic clouds. In NASA Conference Publication, vol. 228 of NASA Conference Publication.

Gopalswamy, N. (2005) Interplanetary Radio Bursts. In Solar and Space Weather Radiophysics: Current Status and Future Developments, edited by D. E. Gary \& C. U. Keller, Springer Netherlands, Dordrecht, pp. 305-333.

Gopalswamy, N. (2010) Corona Mass Ejections: a Summary of Recent Results. In 20th National Solar Physics Meeting, edited by I. Dorotovic.

Gopalswamy, N. (2011) Coronal Mass Ejections and Solar Radio Emissions. Planetary, Solar and Heliospheric Radio Emissions (PRE VII), 325-342.

Gopalswamy, N., Dal Lago, A., Yashiro, S. \& Akiyama, S. (2009) The Expansion and Radial Speeds of Coronal Mass Ejections. Central European Astrophysical Bulletin, 33, 115-124.

Gopalswamy, N., Lara, A., Lepping, R. P., Kaiser, M. L., Berdichevsky, D. \& ST. CyR, O. C. (2000) Interplanetary acceleration of coronal mass ejections. Geophys. Res. Lett., 27, 145-148.

Gopalswamy, N., Lara, A., Yashiro, S., Nunes, S. \& Howard, R. A. (2003a) Coronal mass ejection activity during solar cycle 23. In Solar Variability as an Input to the Earth's Environment, edited by A. Wilson, vol. 535 of ESA Special Publication. 
Gopalswamy, N., Shimojo, M., Lu, W., Yashiro, S., Shibasaki, K. \& HowARD, R. A. (2003b) Prominence Eruptions and Coronal Mass Ejection: A Statistical Study Using Microwave Observations. Astrophys. J., 586, 562-578.

Gopalswamy, N. \& Yashiro, S. (2011) The Strength and Radial Profile of the Coronal Magnetic Field from the Standoff Distance of a Coronal Mass Ejectiondriven Shock. Astrophys. J. Lett., 736, L17.

Gopalswamy, N., Yashiro, S., Kaiser, M. L., Howard, R. A. \& Bougeret, J.L. (2001) Characteristics of coronal mass ejections associated with long-wavelength type II radio bursts. J. Geophys. Res., 106, 29219-29230.

Gopalswamy, N., Yashiro, S., Michaeek, G., Kaiser, M. L., Howard, R. A., Reames, D. V., Leske, R. \& von Rosenvinge, T. (2002) Interacting Coronal Mass Ejections and Solar Energetic Particles. Astrophys. J. Lett., 572, L103-L107.

Gosling, J. T. (1993a) Coronal mass ejections - The link between solar and geomagnetic activity. Physics of Fluids B, 5, 2638-2645.

Gosling, J. T. (1993b) The solar flare myth. J. Geophys. Res., 98, 18937-18950.

Hale, G. E., Ellerman, F., Nicholson, S. B. \& Joy, A. H. (1919) The Magnetic Polarity of Sun-Spots. Astrophys. J., 49, 153-178.

Hayes, A. P., Vourlidas, A. \& Howard, R. A. (2001) Deriving the Electron Density of the Solar Corona from the Inversion of Total Brightness Measurements. Astrophys. J., 548, 1081-1086.

Hildner, E., Gosling, J. T., Macqueen, R. M., Munro, R. H., Poland, A. I. \& Ross, C. L. (1975) The large coronal transient of 10 June 1973. I - Observational description. Solar Phys., 42, 163-177.

Hirayama, T. (1974) Theoretical Model of Flares and Prominences. I: Evaporating Flare Model. Solar Phys., 34, 323-338.

Howard, R. A., Moses, J. D., Vourlidas, A., Newmark, J. S., Socker, D. G., Plunkett, S. P., Korendyke, C. M., Cook, J. W. ET AL. (2008) Sun Earth Connection Coronal and Heliospheric Investigation (SECCHI). Space Sci. Rev., 136, $67-115$.

Howard, R. A., Vourlidas, A., Korendyke, C. M., Plunkett, S. P., Carter, M. T., Wang, D., Rich, N., McMullin, D. R. ET AL. (2013) The solar and heliospheric imager (SoloHI) instrument for the solar orbiter mission. In Society of Photo-Optical Instrumentation Engineers (SPIE) Conference Series, vol. 8862 of Society of Photo-Optical Instrumentation Engineers (SPIE) Conference Series.

HowARD, T. (2011) Coronal mass ejections: an introduction. Springer-Verlag New York. 
HowARD, T. A. \& TAPPIN, S. J. (2009) Interplanetary Coronal Mass Ejections Observed in the Heliosphere: 1. Review of Theory. Space Sci. Rev., 147, 31-54.

Hundhausen, A. J., Sawyer, C. B., House, L., Illing, R. M. E. \& Wagner, W. J. (1984) Coronal mass ejections observed during the solar maximum mission Latitude distribution and rate of occurrence. J. Geophys. Res., 89, 2639-2646.

Kaiser, M. L., Kucera, T. A., Davila, J. M., St. Cyr, O. C., Guhathakurta, M. \& Christian, E. (2008) The STEREO Mission: An Introduction. Space Sci. Rev., 136, 5-16.

Kallenrode, M.-B. (2003) Current views on impulsive and gradual solar energetic particle events. Journal of Physics G: Nuclear and Particle Physics, 29(5), 965.

Kilpua, E. K. J., Pomoell, J., Vourlidas, A., Vainio, R., Luhmann, J., Li, Y., Schroeder, P., Galvin, A. B. ET AL. (2009) STEREO observations of interplanetary coronal mass ejections and prominence deflection during solar minimum period. Annales Geophysicae, 27, 4491-4503.

Kim, R.-S., Gopalswamy, N., Moon, Y.-J., Cho, K.-S. \& Yashiro, S. (2012) Magnetic Field Strength in the Upper Solar Corona Using White-light Shock Structures Surrounding Coronal Mass Ejections. Astrophys. J., 746, 118.

Kopp, R. A. \& Pneuman, G. W. (1976) Magnetic reconnection in the corona and the loop prominence phenomenon. Solar Phys., 50, 85-98.

Landau, L. D. \& Lifshitz, E. M. (1959) Fluid mechanics. Oxford: Pergamon Press, 1959 .

Liu, Y., Davies, J. A., Luhmann, J. G., Vourlidas, A., Bale, S. D. \& Lin, R. P. (2010a) Geometric Triangulation of Imaging Observations to Track Coronal Mass Ejections Continuously Out to 1 AU. Astrophys. J. Lett., 710, L82-L87.

Liu, Y., Luhmann, J. G., Bale, S. D. \& Lin, R. P. (2011) Solar Source and Heliospheric Consequences of the 2010 April 3 Coronal Mass Ejection: A Comprehensive View. Astrophys. J., 734, 84.

Liu, Y., Thernisien, A., Luhmann, J. G., Vourlidas, A., Davies, J. A., Lin, R. P. \& BALE, S. D. (2010b) Reconstructing Coronal Mass Ejections with Coordinated Imaging and in Situ Observations: Global Structure, Kinematics, and Implications for Space Weather Forecasting. Astrophys. J., 722, 1762-1777.

Liu, Y. D., Luhmann, J. G., Lugaz, N., Möstl, C., Davies, J. A., Bale, S. D. \& Lin, R. P. (2013) On Sun-to-Earth Propagation of Coronal Mass Ejections. Astrophys. J., 769, 45.

Liu, Y. D., Luhmann, J. G., Möstl, C., Martinez-Oliveros, J. C., Bale, S. D., Lin, R. P., Harrison, R. A., Temmer, M. et AL. (2012) Interactions between Coronal Mass Ejections Viewed in Coordinated Imaging and in situ Observations. Astrophys. J. Lett., 746, L15. 
Lugaz, N., Hernandez-Charpak, J. N., Roussev, I. I., Davis, C. J., VourliDAS, A. \& Davies, J. A. (2010) Determining the Azimuthal Properties of Coronal Mass Ejections from Multi-Spacecraft Remote-Sensing Observations with STEREO SECCHI. Astrophys. J., 715, 493-499.

Lugaz, N. \& Kintner, P. (2013) Effect of Solar Wind Drag on the Determination of the Properties of Coronal Mass Ejections from Heliospheric Images. Solar Phys., 285, 281-294.

Lugaz, N., Manchester, IV, W. B. \& Gombosi, T. I. (2005) Numerical Simulation of the Interaction of Two Coronal Mass Ejections from Sun to Earth. Astrophys. J., 634, 651-662.

Lugaz, N., Vourlidas, A. \& Roussev, I. I. (2009) Deriving the radial distances of wide coronal mass ejections from elongation measurements in the heliosphere application to CME-CME interaction. Annales Geophysicae, 27, 3479-3488.

Luhmann, J. G., Curtis, D. W., Schroeder, P., McCauley, J., Lin, R. P., Larson, D. E., Bale, S. D., Sauvaud, J.-A. et Al. (2008) STEREO IMPACT Investigation Goals, Measurements, and Data Products Overview. Space Sci. Rev., 136, 117-184.

Maloney, S. A. \& Gallagher, P. T. (2011) STEREO Direct Imaging of a Coronal Mass Ejection-driven Shock to 0.5 AU. Astrophys. J. Lett., 736, L5.

Mann, G., Klassen, A., Aurass, H. \& Classen, H. T. (2003) Development of shocks waves in the solar corona and the interplanetary space. In Solar Wind Ten, edited by M. Velli, R. Bruno, F. Malara \& B. Bucci, vol. 679 of American Institute of Physics Conference Series.

Marubashi, K. (2000) Physics of Interplanetary Magnetic Flux Ropes: Toward Prediction of Geomagnetic Storms. Advances in Space Research, 26, 55-66.

Michalek, G. \& Yashiro, S. (2013) CMEs and active regions on the sun. Advances in Space Research, 52, 521-527.

Moore, R. L. \& Labonte, B. J. (1980) The filament eruption in the 3B flare of July 29, 1973 - Onset and magnetic field configuration. In Solar and Interplanetary Dynamics, edited by M. Dryer \& E. Tandberg-Hanssen, vol. 91 of IAU Symposium.

Möstl, C., Amla, K., Hall, J. R., Liewer, P. C., De Jong, E. M., Colaninno, R. C., Veronig, A. M., Rollett, T. ET Al. (2014) Connecting Speeds, Directions and Arrival Times of 22 Coronal Mass Ejections from the Sun to 1 AU. Astrophys. J., $787(2), 119$.

Möstl, C. \& Davies, J. A. (2013) Speeds and Arrival Times of Solar Transients Approximated by Self-similar Expanding Circular Fronts. Solar Phys., 285, 411423. 
Möstl, C., Temmer, M., Rollett, T., Farrugia, C. J., Liu, Y., Veronig, A. M., Leitner, M., Galvin, A. B. ET Al. (2010) STEREO and Wind observations of a fast ICME flank triggering a prolonged geomagnetic storm on 5-7 April 2010. Geophys. Res. Lett., 37, 24103.

Müller, D., Marsden, R. G., St. Cyr, O. C. \& Gilbert, H. R. (2013) Solar Orbiter . Exploring the Sun-Heliosphere Connection. Solar Phys., 285, 25-70.

Odstrcil, D., Riley, P., Linker, J. A., Lionello, R., Mikic, Z. \& Pizzo, V. J. (2003) 3-D simulations of ICMEs by coupled coronal and heliospheric models. In Solar Variability as an Input to the Earth's Environment, edited by A. Wilson, vol. 535 of ESA Special Publication.

Ontiveros, V. \& Vourlidas, A. (2009) Quantitative Measurements of Coronal Mass Ejection-Driven Shocks from LASCO Observations. Astrophys. J., 693, 267275 .

Park, J., Moon, Y.-J. \& Gopalswamy, N. (2012) Dependence of solar proton events on their associated activities: Coronal mass ejection parameters. Journal of Geophysical Research: Space Physics, 117(A8), n/a-n/a, a08108.

Payne-Scott, R., Yabsley, D. E. \& Bolton, J. G. (1947) Relative Times of Arrival of Bursts of Solar Noise on Different Radio Frequencies. Nature, 160, 256257.

Poomvises, W., Gopalswamy, N., Yashiro, S., Kwon, R.-Y. \& Olmedo, O. (2012) Determination of the Heliospheric Radial Magnetic Field from the Standoff Distance of a CME-driven Shock Observed by the STEREO Spacecraft. Astrophys. J., 758, 118.

Priest, E. R. \& Forbes, T. G. (1990) Magnetic field evolution during prominence eruptions and two-ribbon flares. Solar Phys., 126, 319-350.

Reames, D. V. (1999) Particle acceleration at the Sun and in the heliosphere. Space Sci. Rev., 90, 413-491.

Richardson, I. G., Cane, H. V. \& Cliver, E. W. (2002) Sources of geomagnetic activity during nearly three solar cycles (1972-2000). Journal of Geophysical Research (Space Physics), 107, 1187.

Russell, C. T. (1985) Planetary bow shocks. Washington DC American Geophysical Union Geophysical Monograph Series, 35, 109-130.

Russell, C. T. \& Mulligan, T. (2002) On the magnetosheath thicknesses of interplanetary coronal mass ejections. Planet. Space Sci., 50, 527-534.

SAKURAI, T. (1976) Magnetohydrodynamic interpretation of the motion of prominences. PASJ, 28, 177-198. 
Schwenn, R. (1996) An Essay on Terminology, Myths and Known Facts: Solar Transient - Flare - CME - Driver Gas - Piston - BDE - Magnetic Cloud - Shock Wave Geomagnetic Storm. Ap\&SS, 243, 187.

Schwenn, R., Dal Lago, A., Huttunen, E. \& Gonzalez, W. D. (2005) The association of coronal mass ejections with their effects near the Earth. Annales Geophysicae, 23, 1033-1059.

SeIfF, A. (1962) Gas Dynamics in Space Exploration. In The NASA University Conference on the Science and Technology of Space Exploration, vol. 24 of NASA Special Publication.

Sheeley, N. R., Walters, J. H., Wang, Y.-M. \& Howard, R. A. (1999) Continuous tracking of coronal outflows: Two kinds of coronal mass ejections. J. Geophys. Res., 104, 24739-24768.

Sheeley, N. R., Wang, Y.-M., Hawley, S. H., Brueckner, G. E., Dere, K. P., Howard, R. A., Koomen, M. J., Korendyke, C. M. et Al. (1997) Measurements of Flow Speeds in the Corona Between 2 and $30 \mathrm{R}_{\odot}$. Astrophys. J., 484, 472-478.

Sheeley, Jr., N. R., Herbst, A. D., Palatchi, C. A., Wang, Y.-M., Howard, R. A., Moses, J. D., Vourlidas, A., Newmark, J. S. ET Al. (2008) Heliospheric Images of the Solar Wind at Earth. Astrophys. J., 675, 853-862.

Sheeley, Jr., N. R., Howard, R. A., Michels, D. J., Koomen, M. J., Schwenn, R., Muehlhaeuser, K. H. \& Rosenbauer, H. (1985) Coronal mass ejections and interplanetary shocks. J. Geophys. Res., 90, 163-175.

Sime, D. G. \& Hundhausen, A. J. (1987) The coronal mass ejection of July 6, 1980 - A candidate for interpretation as a coronal shock wave. J. Geophys. Res., 92, 1049-1055.

Skála, J., Baruffa, F., Büchner, J. \& RAmpp, M. (2015) The 3D MHD code GOEMHD3 for astrophysical plasmas with large Reynolds numbers. Code description, verification, and computational performance. Astron. Astrophys., 580, A48.

Smith, Z. \& DRYeR, M. (1990) MHD study of temporal and spatial evolution of simulated interplanetary shocks in the ecliptic plane within 1 AU. Solar Phys., 129, 387-405.

Spreiter, J. R. \& Stahara, S. S. (2013) Magnetohydrodynamic and Gasdynamic Theories for Planetary Bow Waves. In Collisionless Shocks in the Heliosphere: Reviews of Current Research, edited by B. T. Tsurutani \& R. G. Stone, American Geophysical Union, pp. 85-107.

Spreiter, J. R., Summers, A. L. \& Alksne, A. Y. (1966) Hydromagnetic flow around the magnetosphere. Planet. Space Sci., 14, 223. 
Spruit, H. C. (2013) Essential Magnetohydrodynamics for Astrophysics. ArXiv eprints.

Stone, E. C., Frandsen, A. M., Mewaldt, R. A., Christian, E. R., MarGolies, D., Ormes, J. F. \& Snow, F. (1998) The Advanced Composition Explorer. Space Sci. Rev., 86, 1-22.

Sturrock, P. A. (1966) Model of the High-Energy Phase of Solar Flares. Nature, 211, 695-697.

Tappin, S. J. (2006) The Deceleration of an Interplanetary Transient from the Sun to 5 Au. Solar Phys., 233, 233-248.

Thernisien, A. (2011) Implementation of the Graduated Cylindrical Shell Model for the Three-dimensional Reconstruction of Coronal Mass Ejections. ApJS, 194, 33.

Thernisien, A. F. R., Howard, R. A. \& Vourlidas, A. (2006) Modeling of Flux Rope Coronal Mass Ejections. Astrophys. J., 652, 763-773.

Thompson, B. J., Plunkett, S. P., Gurman, J. B., Newmark, J. S., St. Cyr, O. C. \& Michels, D. J. (1998) SOHO/EIT observations of an Earth-directed coronal mass ejection on May 12, 1997. Geophys. Res. Lett., 25, 2465-2468.

Tripathi, D., Bothmer, V. \& Cremades, H. (2004) The basic characteristics of EUV post-eruptive arcades and their role as tracers of coronal mass ejection source regions. Astron. Astrophys., 422, 337-349.

UCHIDA, Y. (1960) On the Exciters of Type II and Type III Solar Radio Bursts. PASJ, 12,376 .

Verigin, M., Slavin, J., Szabo, A., Kotova, G. \& Gombosi, T. (2003) Planetary bow shocks: Asymptotic MHD Mach cones. Earth, Planets, and Space, 55, 33-38.

Volpes, L. \& Bothmer, V. (2015) An Application of the Stereoscopic Self-SimilarExpansion Model to the Determination of CME-Driven Shock Parameters. ArXiv e-prints.

Vourlidas, A. (2006) Detections of CME-Driven Shocks with LASCO. In SOHO-17. 10 Years of SOHO and Beyond, vol. 617 of ESA Special Publication.

Vourlidas, A. \& Bemporad, A. (2012) A decade of coronagraphic and spectroscopic studies of CME-driven shocks. In American Institute of Physics Conference Series, edited by J. Heerikhuisen, G. Li, N. Pogorelov \& G. Zank, vol. 1436 of American Institute of Physics Conference Series.

Vourlidas, A., Buzasi, D., Howard, R. A. \& Esfandiari, E. (2002) Mass and energy properties of LASCO CMEs. In Solar Variability: From Core to Outer Frontiers, edited by A. Wilson, vol. SP-506 of ESA Conference Proceedings, ESA, Noordwijk. 
Vourlidas, A., Howard, R. A., Plunkett, S. P., Korendyke, C. M., Thernisien, A. F. R., Wang, D., Rich, N., Carter, M. T. et Al. (2015) The Wide-Field Imager for Solar Probe Plus (WISPR). Space Sci. Rev..

Vourlidas, A. \& Ontiveros, V. (2009) A Review of Coronagraphic Observations of Shocks Driven by Coronal Mass Ejections. In American Institute of Physics Conference Series, edited by X. Ao \& G. Z. R. Burrows, vol. 1183 of American Institute of Physics Conference Series.

Vourlidas, A., Subramanian, P., Dere, K. P. \& Howard, R. A. (2000) LargeAngle Spectrometric Coronagraph Measurements of the Energetics of Coronal Mass Ejections. Astrophys. J., 534, 456-467.

Vourlidas, A., Wu, S. T., Wang, A. H., Subramanian, P. \& Howard, R. A. (2003) Direct Detection of a Coronal Mass Ejection-Associated Shock in Large Angle and Spectrometric Coronagraph Experiment White-Light Images. Astrophys. J., 598, 1392-1402.

WANG, Y.-M. (2000) EIT Waves and Fast-Mode Propagation in the Solar Corona. Astrophys. J. Lett., 543, L89-L93.

WebB, D. F. \& Howard, T. A. (2012) Coronal Mass Ejections: Observations. Living Reviews in Solar Physics, $\mathbf{9}(3)$.

Webb, D. F., Howard, T. A., Fry, C. D., Kuchar, T. A., Odstrcil, D., Jackson, B. V., Bisi, M. M., Harrison, R. A. ET Al. (2009) Study of CME Propagation in the Inner Heliosphere: SOHO LASCO, SMEI and STEREO HI Observations of the January 2007 Events. Solar Phys., 256, 239-267.

WebB, D. F. \& Hundhausen, A. J. (1987) Activity associated with the solar origin of coronal mass ejections. Solar Phys., 108, 383-401.

Wild, J. P. \& MCCREAdy, L. L. (1950) Observations of the Spectrum of HighIntensity Solar Radiation at Metre Wavelengths. I. The Apparatus and Spectral Types of Solar Burst Observed. Australian Journal of Scientific Research A Physical Sciences, 3, 387.

Wills-Davey, M. J., DeForest, C. E. \& Stenflo, J. O. (2007) Are "EIT Waves" Fast-Mode MHD Waves? Astrophys. J., 664, 556-562.

Yashiro, S., Gopalswamy, N., Akiyama, S., Michalek, G. \& Howard, R. A. (2005) Visibility of coronal mass ejections as a function of flare location and intensity. Journal of Geophysical Research (Space Physics), 110(A9), A12S05.

Yashiro, S., Gopalswamy, N., Michalek, G., St. Cyr, O. C., Plunkett, S. P., Rich, N. B. \& Howard, R. A. (2004) A catalog of white light coronal mass ejections observed by the SOHO spacecraft. Journal of Geophysical Research (Space Physics), 109, 7105. 
Zhang, J. \& Dere, K. P. (2006) A Statistical Study of Main and Residual Accelerations of Coronal Mass Ejections. Astrophys. J., 649, 1100-1109.

Zhang, J., Dere, K. P., Howard, R. A., Kundu, M. R. \& White, S. M. (2001) On the Temporal Relationship between Coronal Mass Ejections and Flares. Astrophys. J., 559, 452-462.

Zhang, J., Dere, K. P., Howard, R. A. \& Vourlidas, A. (2004) A Study of the Kinematic Evolution of Coronal Mass Ejections. Astrophys. J., 604, 420-432.

Zhao, X. P., Plunkett, S. P. \& Liu, W. (2002) Determination of geometrical and kinematical properties of halo coronal mass ejections using the cone model. J. Geophys. Res., 107, 1223. 


\section{Acknowledgements}

First and foremost I would like to thank Dr. V. Bothmer andProf. A. Reiners for the helpful supervision and the always understanding attitude. I am extremely grateful for their support, as well as for the help that they provided together with Prof. S. Dreizler and Ms S. Bertram.

Thanks to the members of the committee and to Prof. J. Büchner for agreeing to review this thesis. Thanks to my family and friends, and thanks to my partner Dimitar, I will be forever grateful for your help.

Thanks to Jackie Davies and Richard Harrison from Rutherford Appleton Laboratory, Didcot, Chilton, UK, for providing support in the processing of STEREO/SECCHI images and time-elongation plots.

This work was funded by the Deutsche Forschungsgemeinschaft (DFG) under grant SFB 963/1, projects A2 and A4. 



\title{
Curriculum Vitae
}

\author{
Name: Volpes, Laura \\ Place of birth: Marino (RM), Italy \\ Date of birth: 07.09.1985 \\ Nationality: Italian
}

\section{University Qualifications:}

$09 / 2012$ - present

$2010-2012$

$2004-2010$

\section{Doctoral Studies}

Institute of Astrophysics, University of Göttingen

Thesis: On the interplanetary properties and evolution of CME-driven shocks

Master of Science in Astrophysics

Astromundus Masters Course

Study path through the Universities of Innsbruck, Padova and Göttingen

Thesis at University of Göttingen: Thermal and magnetic structure of the solar corona in a 3D MHD simulation

Bachelor degree in Physics

Universitá degli Studi di Roma Tor Vergata, Italy

Study path through the Universities of Innsbruck, Padova and Göttingen

Thesis: Numerical simulations of flux transport across the photosphere

\section{Education:}

1999 - 2004 Scientific High School Bruno Touschek, Grottaferrata (RM), Italy

High School Diploma

Göttingen, 24.05.2016 